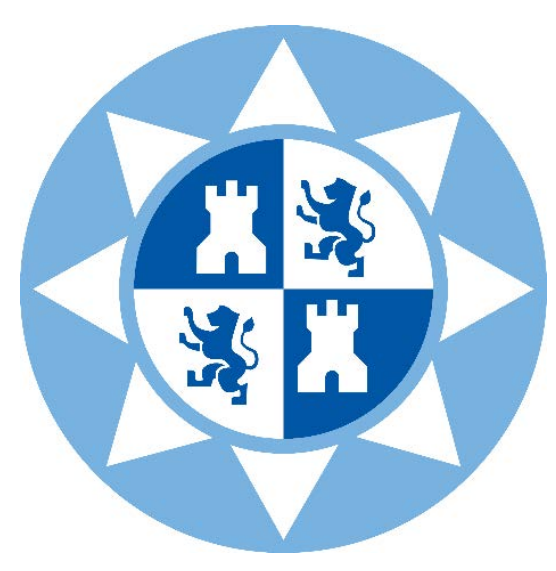

UNIVERSIDAD POLITÉCNICA DE CARTAGENA

DEPARTAMENTO DE TECNOLOGÍAS DE LA INFORMACIÓN Y LAS COMUNICACIONES

\title{
Contribución al desarrollo de sistemas automáticos de planificación eficiente de rutas
}

SERGIO LUJÁN FERNÁNDEZ 


\section{Agradecimientos}

A mi esposa Nuria, por su apoyo incondicional y por aportar tanta felicidad a mi vida. También a mi padre, por sus enseñanzas y experiencias inculcadas desde pequeño y que tanto me han ayudado en mi desarrollo profesional. A mi familia, madre y hermanas, por el cariño que me han transmitido desde que nací. Y a mis padres políticos, por hacerme sentir como un hijo más.

Por último, a mis directores Fernando y Juan, por su empeño y ayuda para hacer realidad esta tesis. Y a mi amigo y compañero Andrés, por todo el trabajo que hemos llevado a cabo juntos. 


\section{Resumen}

El objetivo de esta tesis doctoral es el desarrollo de un sistema de detección de dispositivos móviles y su aplicación en los sistemas de planificación eficiente de rutas. Para ello, se lleva a cabo la implementación piloto de un prototipo de sistema global automático para la estimación de información origen-destino (OD) en los medios públicos de transporte. Esta herramienta TIC de medición está basada en los resultados del análisis de la demanda y el flujo de pasajeros en las líneas urbanas de transporte, sus tasas de ocupación y las matrices origen-destino. Esto se logra mediante la detección de dispositivos electrónicos personales a bordo (etiquetas Bluetooth o WiFi), el procesamiento de esa información y su transmisión. Las muestras detectadas, tras ser tratadas por un procesador estadístico de decisión para determinar cuáles de ellas son representativas y cuáles no, permiten la creación de patrones de comportamiento atendiendo a las matrices de probabilidades OD resultantes. La capacidad de este prototipo para la obtención de grandes volúmenes de muestras, ha permitido garantizar la viabilidad de predicción del sistema con porcentajes de error despreciables. Este trabajo de tesis permite la mejora del servicio prestado por las empresas de transporte público de pasajeros a sus clientes directos, el consorcio de transportes de las ciudades en las que operan, así como los usuarios finales, los pasajeros, lo que facilita una movilidad más inteligente y sostenible. 


\section{Abstract}

The aim of this thesis is to develop a detection system of mobile devices and their application in efficient route planning. To this end, a pilot is carried out with an automated system prototype for estimating overall information origindestination (OD) in public transport services. This ICT measurement tool is based on the results of the analysis of demand and passenger flow in urban transport lines, occupancy rates and origin-destination matrices. This is accomplished by detecting personal electronic devices on board (Bluetooth or WiFi labels), processing and transmitting such information. The samples detected, after being treated by a statistical decision processor to determine which of these are representative and which are not, allow the creation of patterns of behavior in response to the OD resulting probability matrices. The capacity of this prototype for the production of large volumes of samples, has ensured the system viability for prediction with negligible percentages error. This thesis can improve the service provided by public transport companies of passengers to their direct customers, the transport consortium of cities in which they operate, as well as end users, the passengers, facilitating a smarter mobility and sustainable. 


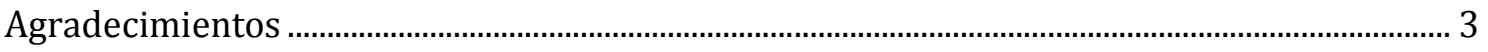

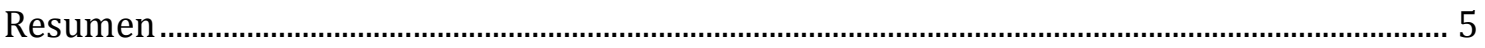

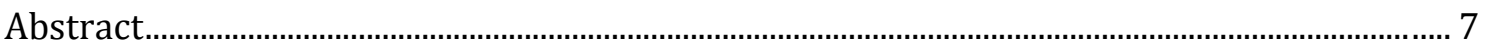

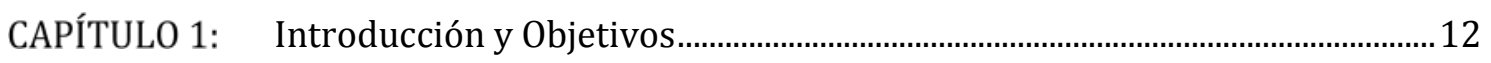

1.1 Motivación..................................................................................................................... 12

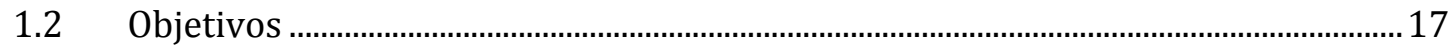

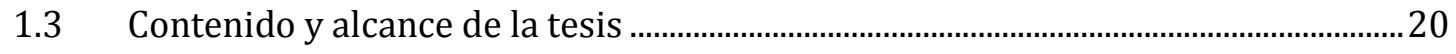

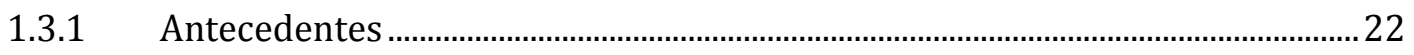

1.3.2 Justificación de la necesidad del trabajo de tesis como solución al problema planteado.

1.3.3 Descripción en relación al problema planteado del estado de la tecnología en España y en el extranjero, y como el sistema propuesto va más allá del estado del arte. 26

1.3.4 Resumen técnico de las principales actividades del trabajo de tesis ................27

1.3.4.1 Adquisición, filtrado y transmisión...................................................................27

1.3.4.1.1 Detección de dispositivos y adquisición de datos ......................................28

1.3.4.1.2 Filtrado de datos .............................................................................................29

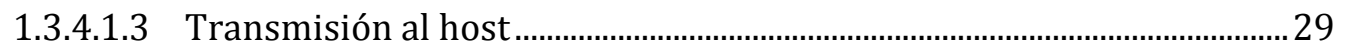

1.3.4.2 Simulación, conteo y predicción...........................................................................

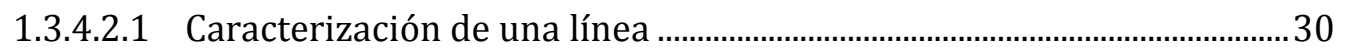

1.3.4.2.2 Simulación de una línea de autobús a partir de la generación estocástica de pasajeros................................................................................................... 30

1.3.4.2.3 Estimación de la Matriz O-D - Planificación ...................................................31

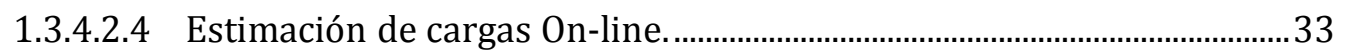

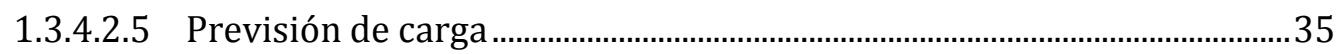

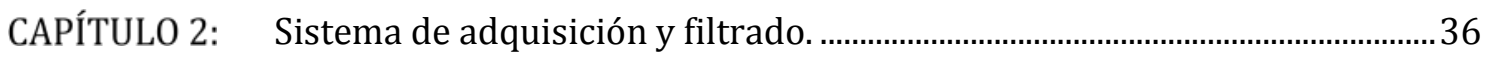

2.1 Estado del arte de los sistemas de localización en entornos Indoor...........................37

2.1.1 Soluciones con estándar Bluetooth .........................................................................37

2.1.2 Soluciones con estándar Wifi......................................................................................38

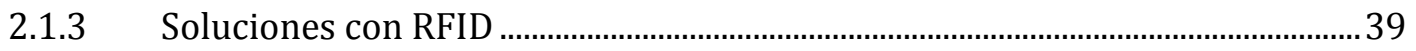

2.1.4 Soluciones con estándar ZigBee.................................................................................40

2.2 Propuesta de un sistema concreto …………................................................................ 40

2.2.1 Descripción general del sistema ………............................................................. 40

2.2.1.1 Arquitectura y estructura del sistema.................................................................4

2.2.1.2 Estaciones detectoras.......................................................................................... 43 
2.2.1.3 Servidor de monitorización .................................................................................. 44

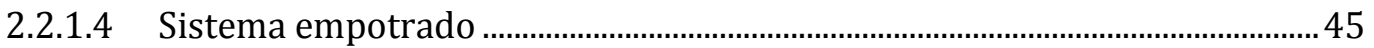

2.2.2 Programación de la aplicación de detección de las estaciones............................46

2.2.2.1 Detecciones BT.................................................................................................... 47

2.2.2.2 Socket TCP para envió de la información............................................................. 48

2.2.2.3 Aspectos críticos..................................................................................................... 48

2.2.2.4 Compilación de la aplicación ........................................................................... 49

2.2.3 Preparación de los empotrados .......................................................................50

2.2.3.1 Activación de los dispositivos BT y WIFI USB....................................................51

2.2.3.2 Configuración del dispositivo BT y WIFI USB .....................................................53

2.2.3.3 Configuración de la aplicación en el empotrado ................................................56

2.2.4 Software de monitorización ....................................................................................57

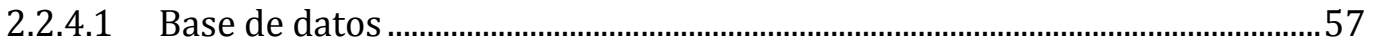

2.2.4.2 Interfaz gráfica .................................................................................................60

2.2.4.3 Gestión concurrente de los sockets ..................................................................61

2.2.4.4 Procesado de la información ...............................................................................61

2.3 Prueba y Evaluación del sistema................................................................................62

$2.4 \quad$ Uso del sistema para control de asistencia en entornos docentes............................... 70

2.4.1 Tecnologías implementadas ................................................................................

2.4.1.1 Seguridad e identificación ............................................................................... 72

2.4.2 Más allá del control de acceso ……………………………………………........... 73

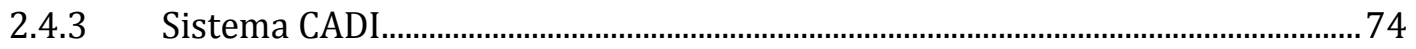

2.4.3.1 Integración de componentes.......................................................................... 74

2.4.3.2 Arquitectura software .......................................................................................

CAPÍTULO 3: Sistema de estimación de Matrices Origen-Destino ............................................77

3.1 Sistema de adquisición de datos adaptado a la red de transporte...............................78

$3.2 \quad$ Matrices Origen-Destino..................................................................................................... 88

3.3 Escenario de capturas: Prueba piloto …………........................................................90

3.4 Modelado del conjunto de terminales Bluetooth activos.............................................91

3.4.1 Propiedades de la variable aleatoria de Poisson ……………………………..........92

3.4.2 Aplicación a les matrices OD ........................................................................................ 95

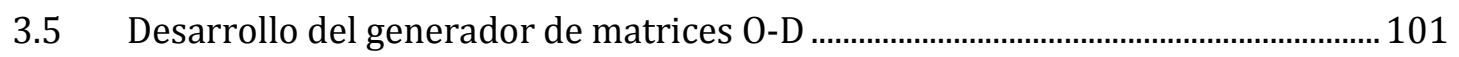

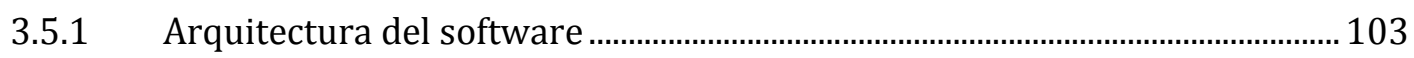

3.5.1.1 Análisis y diseño de la base de datos. ............................................................ 110

3.5.2 Software de simulación ................................................................................... 111 
3.5.2.2 Desarrollo del software ................................................................................... 113

3.5.2.3 Control de Usuario (Sesiones web) ................................................................... 114

3.5.2.3.1 Subir archivos (*.Txt) _................................................................................. 116

3.5.2.3.2 Procesado de la información.......................................................................... 117

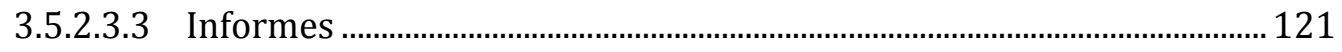

CAPÍTULO 4: Sistema alternativo de comunicaciones entre equipos embarcados de las

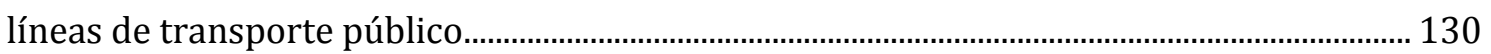

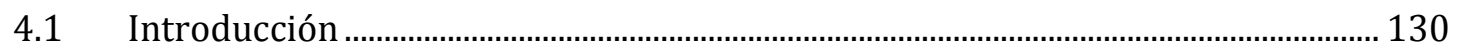

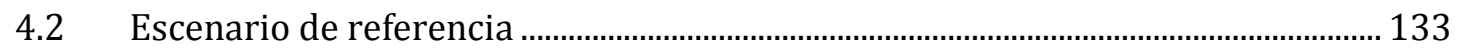

$4.3 \quad$ Especificación del protocolo ......................................................................................... 134

4.4 Descripción del funcionamiento en cada caso de uso ................................................. 137

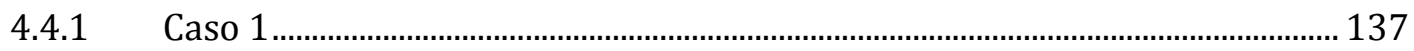

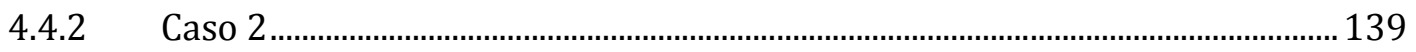

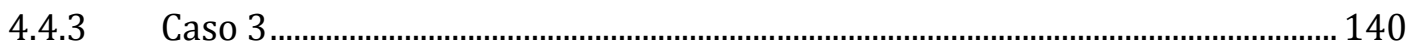

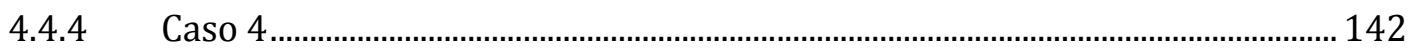

4.4.5 Mejora del tráfico cursado en la red de comunicaciones con el uso del protocolo propuesto ............................................................................................................... 144

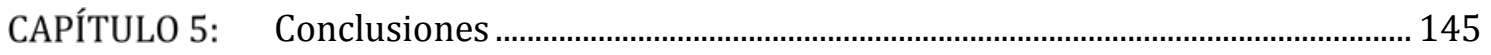

CAPÍTULO 6: BIBLIOGRAFÍA Y REFERENCIAS........................................................................ 150 


\section{CAPÍTULO 1: Introducción y Objetivos}

El trabajo de tesis con carácter innovador que se presenta a continuación está destinado a contribuir en la mejora de las tecnologías de la información en importantes sectores logísticos de ámbito internacional, como el transporte público. Se ha llevado a cabo un profundo estudio de las posibles soluciones a desarrollar a partir de un análisis de necesidades, restricciones e incompatibilidades que dificultan la existencia de tecnología eficiente en este ámbito. El principal objetivo se centra en el desarrollo de un sistema basado en tecnologías y herramientas estadísticas que permitan obtener patrones realistas del comportamiento cotidiano de la población en determinadas situaciones. Tal información, adquirida por procedimientos de sondeo, de modo anónimo, sin la necesidad de interferir en la privacidad de las personas y sin llegar a tener que interactuar con ellas, es finalmente procesada en términos estadísticos.

Existen multitud de líneas de trabajo relacionadas con la detección e identificación de personas, siendo éstas el punto de partida de esta investigación. Desde los sistemas de procesamiento digital de imágenes hasta los sistemas de detección que utilizan ondas electromagnéticas para la detección de personas, existe un amplio abanico tecnológico que servirá como base para el desarrollo de una solución equilibrada. Se contemplará la validación de los resultados obtenidos con las diferentes alternativas tecnológicas.

La idoneidad de los datos o muestras que se puedan obtener a partir de las distintas soluciones vendrá estrechamente ligada al procesamiento estadístico que pueda llevarse a cabo con los mismos. Se estudiarán los procesos estadísticos que sirvan para extraer conclusiones relevantes y se llevará a cabo el desarrollo de un procesador que permita validará con los resultados reales y se utilizará para estudiar la viabilidad de hipótesis de trabajo relacionadas con la planificación de rutas.

\subsection{Motivación}

Hoy en día, multitud de sectores comerciales demandan grandes volúmenes de información referente a las necesidades y exigencias de clientes potenciales. En el mundo de los negocios se habla de que "la información es poder", pero no es la 
información en sí lo que da el poder, sino los recursos y habilidades necesarios para obtenerla a tiempo y mantenerla actualizada.

Las TIC juegan un papel crucial en este sentido. Desde hace más de medio siglo se han venido aplicando tecnologías para agilizar la adquisición de datos y reducir el coste de los mismos. Entonces, la aparición del lector de códigos de barras supuso una revolución para empresas y comercios. En los últimos años, el uso de bases de datos y las numerosas contribuciones científicas han servido para incorporar importantes novedades tecnológicas, como la identificación por radiofrecuencia (RFID).

La línea de trabajo que se propone atiende a tipos información cuyo principal hándicap es su coste de adquisición. Hay que ser consciente de que, dentro de la información útil que puede ser demandada para optimizar las estrategias de negocio, hay tipos de datos o muestras que sólo pueden ser obtenidos utilizando herramientas cuyos costes no hacen rentable su adquisición. Entre los muestreos que más dificultades ofrecen se encuentran los que requieren sondeos sobre grandes masas humanas. Estos suelen llevarse a cabo con el fin de agrupar perfiles humanos de comportamiento, necesidades y actitudes similares. En este tipo de información se basa principalmente el marketing relacional.

El marketing relacional tiene como objetivo identificar los perfiles de cliente más rentables para actuar en consecuencia, descubriendo sus necesidades y manteniendo una evolución de los productos o servicios de acuerdo con ellos a lo largo del tiempo. En algunas situaciones, la información puede ser obtenida haciendo uso de recursos verdaderamente rentables. Por ejemplo, las tarjetas de fidelización, que son lanzadas por grandes líneas aéreas, cadenas textiles y de alimentación. En cambio, existen otras situaciones en las que no puede satisfacerse la demanda de información utilizando esta metodología dado que no se existe una interacción con el cliente al pasar por la caja registradora o por una puerta de embarque, como ocurre en tiendas o aeropuertos. En estos casos se ha de recurrir a métodos muy costosos, como pueden ser las encuestas o la observación directa, que además no contemplan la posibilidad de un sondeo continuo en el tiempo. En este periodo investigador se ha atendido la problemática tecnológica que dificulta la adquisición de información de este tipo. 
Existen multitud de datos que pueden ser recogidos de forma sencilla, como por ejemplo el número de personas que viaja a bordo de un autobús urbano. Bastaría con utilizar el sistema de validación o billetaje del autobús, un sistema de altas prestaciones sólo para lo que está diseñado: el control y la supervisión de acceso a bordo del pasaje. Este sistema no contempla el conteo de bajadas, por lo que es insuficiente para determinar el número de pasajeros a bordo. Para conseguir esto último sin necesidad de alterar la rutina habitual del usuario del bus y sin que tenga que llevar consigo ningún dispositivo adicional para ello, bastaría con colocar algún otro sistema de conteo de bajada, sensores de presión en los asientos, sensores ópticos, incluso sistemas de procesado digital de imágenes. Pero, además de que cualquiera de estas incorporaciones supondría un problema económico, ninguna de ellas ofrecería información suficiente como para saber que el usuario que subió en la parada $\mathrm{X}_{1}$ a la hora $\mathrm{Y}_{1}$, bajó en la parada $\mathrm{X}_{2}$ a la hora $\mathrm{Y}_{2}$.

Como se puede observar, existe otro tipo de información cuya obtención no es trivial, aunque la posibilidad de disponer de ella sin elevados costes permitiría introducir importantes mejoras para el negocio. En el ejemplo del párrafo anterior, la información de parada y hora de subida y de bajada de cada usuario, procesada en tiempo real mediante algoritmos estadísticos y de toma de decisiones, daría cabida a mejorar notablemente la calidad del servicio:

- Facilitaría la coordinación de las líneas.

- Posibilitaría la detección de incidencias.

- Agilizaría la adaptabilidad ante imprevistos.

- Permitiría publicar información inmediata sobre el estado y disponibilidad de los itinerarios.

De este modo, la rentabilidad de la red de transporte público se vería incrementada de forma considerable.

En este sentido, son numerosas las empresas cuyos departamentos logísticos o de marketing demandarían una información que a día de hoy no barajan. En consecuencia, esta necesidad por descubrir debía ser atendida desde un punto de vista científico en el marco de las tecnologías de la información y las comunicaciones. 
La solución a desarrollar para la obtención de esta información está compuesta por dos partes bien diferenciadas, pero dependientes la una de la otra. Por un lado se encuentra la adquisición de datos, los cuales podrán ser más, menos, mejores, peores, en mayor cantidad y en menor cantidad, en función de la tecnología en la que se base su adquisición. Por otro lado, es necesario un procesado de toda la información para la extracción de resultados depurados, precisos y concisos.

A continuación se enumeran las dos alternativas tecnológicas evaluadas y más adecuadas para la adquisición de datos, que sirvieron como punto de partida para esta parte de la investigación.

- Procesamiento de imágenes: se trata de muestrear una imagen facial y realizar una codificación que permita su posterior comparación mediante algoritmos de decisión con una segunda imagen también muestreada y codificada siguiendo el mismo patrón. En el caso del ejemplo del bus urbano, una fotografía de la cara del usuario al subir se compararía con la de los que bajan con el fin de disponer de una muestra con las variables $\left[\mathrm{X}_{1}, \mathrm{Y}_{1}, \mathrm{X}_{2}, \mathrm{Y}_{2}\right]$. Esta opción cuenta con la ventaja de que, al tener un código por cada usuario embarcado, cuando se obtiene el código de la persona que sale, sólo se tendrá que comparar éste con los códigos de las personas que se encontraban embarcadas en ese momento, y se decidirá por similitud a cuál corresponde. Como desventaja destaca la complejidad en el diseño de la algorítmica de codificación para que dos fotos de la cara de una misma persona en distinta posición dentro del encuadre de la fotografía generen códigos similares. Conlleva también altas cargas de computación a la hora de responder ante grandes volúmenes de personas.

- Detección de dispositivos electrónicos que las personas suelen llevar consigo (móviles, PDAs, portátiles, etc.). Esta opción es la que se describe a lo largo de este trabajo de tesis como solución óptima para la obtención de las muestras precisas demandadas y que también tendrá aplicación en otros ámbitos de negocio. Se basa en la utilización de sistemas de comunicación por medio de ondas electromagnéticas, partiendo de 
variantes de los estándares inalámbricos más comunes (WiFi, Bluetooth, etc.).

En función de las alternativas anteriores y de la calidad de las muestras extraídas, el procesado automático de las mismas contempla las siguientes etapas:

- Eliminación de muestras poco representativas según parámetros de decisión.

- Identificación de tendencias y patrones significativos que retroalimenten el sistema con el fin de ajustar los parámetros de decisión.

- Organización de la información en matrices origen-destino.

- Cálculo de matrices de probabilidad.

- Representación de resultados en matrices de probabilidad acumulativas.

Para solventar los problemas de decisión se estudiaron las reglas heurísticas en las que se basa la lógica difusa.

Es fundamental encontrar una solución (Fig.1) que equilibre las condiciones de adquisición de las muestras con su procesado. Para ello debe existir una realimentación que permita modificar los parámetros muestreo.

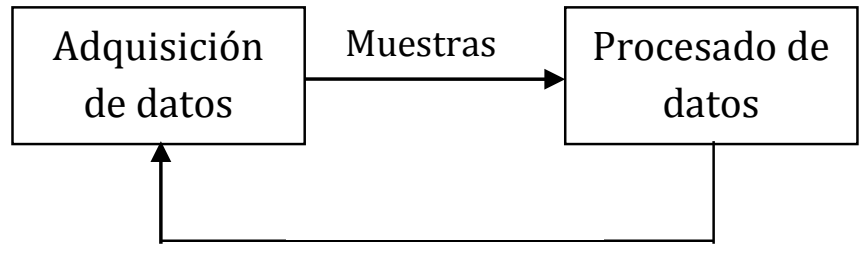

Figura 1. Flujograma de solución recomendado

Desde el principio de este trabajo, se vio que existía información muy útil que se podía obtener a partir de grandes cantidades de muestras procesadas en tiempo real, y que la existencia de un dispositivo para su adquisición de forma rentable podría llegar a convertirse en una herramienta básica que mejorase de forma sustancial las decisiones estratégicas de empresas de multitud de sectores, contribuyendo a que pudieran mantenerse en una posición más competitiva, sobretodo en tiempos de crisis. 


\subsection{Objetivos}

El principal objetivo de este trabajo de tesis es el desarrollo de una plataforma tecnológica destinada a la realización de sondeos basados en la presencia de personas en determinados emplazamientos. La idea es poder detectar cuándo una persona entra a una zona controlada y cuándo se marcha, sin llegar a alterar de ningún modo su rutina y sin la necesidad de portar con ningún dispositivo adicional. Para ello, el desarrollo que se describe en el presente documento se llevará a cabo aplicado a la optimización de las líneas de transporte público con el fin de evaluar las soluciones para un caso concreto $y$, a su vez, poder validar los resultados estimados con los reales.

A continuación se describen los objetivos iniciales planteados: OBJETIVO 1.

CONTRIBUIR AL DESARROLLO DE TECNOLOGÍAS EFICIENTES PARA LA ADQUISICIÓN DE INFORMACIÓN RELEVANTE DE CARA A LA MEJORA DE ESTRATEGIAS COMERCIALES

\section{Novedad:}

Existe en la literatura científica una gran variedad de sistemas de detección y localización basados en diversas tecnologías, como WiFi [Zaruba'2007], RFID [Tesoriero'2008], Bluetooth (BT) [Peterson'2006] o combinaciones de ellas [Aparicio'2008][Hii'2005][Matthieu'2005], las cuales han sido barajadas inicialmente para ser utilizadas a modo de sensor o baliza para estimar la presencia de personas en un lugar concreto. Ninguna de estas opciones ofrece la posibilidad de extraer la información completa bajo las condiciones que se pretenden: RFID implica que los usuarios lleven consigo una etiqueta para poder ser detectados y las soluciones WiFi y BT implican la conexión voluntaria con APs. Este objetivo pretende cubrir esta carencia tecnológica contribuyendo al desarrollo de nuevas soluciones basadas en sensores capaces de, no sólo discernir unívocamente una persona de otra, sino también hacerlo de forma discreta y transparente para el usuario.

\section{Relevancia:}


Desde el punto de vista del procesado digital de imágenes [Bradski'2008] se abre la posibilidad del reconocimiento facial que permita construir la muestra con la información deseada. En esta línea, las técnicas de procesado de imágenes permitirían obtener resultados de impacto. Del mismo modo, tiene relevancia el desarrollo de alternativas basadas en la interacción con los dispositivos de comunicación inalámbricos disponibles en los terminales portables que los usuarios llevan consigo. Por ejemplo, diseñando metodologías para la detección de dispositivos Bluetooth, disponibles en los terminales de un alto porcentaje de la población, sin la necesidad de interaccionar con el usuario [Lujan'2008] [Lujan'2011].

OBJETIVO 2.

\section{DESARROLLAR UNA SOLUCIÓN ADAPTADA A LA OPTIMIZACIÓN DE LÍNEAS DE TRANSPORTE PÚBLICO}

\section{Novedad:}

En el sector del transporte público, las opciones tecnológicas adoptadas para evitar los costosos conteos manuales (encuestadores apostados en las puertas de los vehículos o en las salidas de las dársenas o andenes) son: las alfombras sensibles a la presión en las entradas y salidas de los autobuses, microbarómetros en los neumáticos o sensores ópticos y térmicos. Pero en ningún caso proporcionan información origen-destino del usuario. La solución que se pretende desarrollar introduce como elemento diferencial la posibilidad de determinar la parada y la hora en la que un usuario sube al autobús, y la parada y la hora en la que baja. Estos datos son de vital importancia a la hora de conocer la movilidad asociada a una línea, estimar niveles de ocupación, prever posibles incidencias, etcétera.

\section{Relevancia:}

Se deberán desarrollar modelos de clasificación basados en lógica difusa o inteligencia artificial [Zheng'2002] [Khanam'2008]. Las entradas serán los datos extraídos de las muestras tras un procesado previo. Este preprocesado de datos tiene la finalidad de adecuar el flujo de datos a la capacidad de 
cómputo del sistema en tiempo real. Como salidas se obtendrán patrones realistas de comportamiento asociados a matrices de probabilidad OrigenDestino. Será necesario analizar estadísticamente los márgenes de error para consensuar mejoraras en la precisión del sistema. Para ello, se diseñará un software que permita adecuar los parámetros de procesamiento de datos a la metodología de adquisición. Finalmente, se validarán los módulos desarrollados con objeto de obtener resultados de impacto y se evaluarán las posibles patentes.

Algunas consideraciones básicas a tener en cuenta para el desarrollo del sistema automático de planificación de rutas propuesto son:

- Los sistemas de adquisición de datos no precisarán la interacción del individuo ni degradarán los servicios que se le estén prestando.

- Los sondeos deberán realizarse de forma individualizada y anónima. Es decir, tener la certeza de que la persona que acaba de salir es la que entró en un instante determinado y no otra, sin la necesidad de identificarla mediante datos personales.

- Se precisará la viabilidad de sondeo de forma continuada en lugares de pública concurrencia y sobre grandes masas humanas.

- Deberá existir una adaptación a la algorítmica de toma de decisiones y clasificación que permita agrupar perfiles sujetos a determinadas condiciones, con el fin de eliminar las muestras que aporten información redundante.

- El sistema deberá garantizar un intervalo de trabajo mínimo que asegure su adaptabilidad a las especificaciones de cada caso (condiciones de adquisición de la información y adecuación de la misma para su procesado, tolerancias permitidas, formas de representación de resultados, etcétera).

- Deberán idearse mecanismos alternativos de validación de resultados: los simuladores permitirán crear escenarios con alto grado de semejanza.

- Las propuestas deben ser de carácter innovador y de relevancia científica. 
- Es requisito indispensable tener en cuenta la rentabilidad de los diseños en función del valor estimado de la información que se pretenda obtener.

\subsection{Contenido y alcance de la tesis}

El trabajo de tesis ha concluido con el desarrollo de un prototipo y puesta en práctica de un piloto de validación de una plataforma que se puede denominar como sistema global de conteo automático de pasajeros a bordo de una línea de autobuses, con información origen-destino. Este sistema estima la matriz de movilidad O-D de una línea de transporte público, y determina el grado de ocupación en tiempo real, además de una previsión de carga a bordo con el objetivo de realizar una planificación eficaz de rutas. Todo ello se consigue mediante la detección, y conveniente procesamiento y transmisión de la información detectada, de los dispositivos emisores de radiofrecuencia (wifi o bluetooth) que los pasajeros suelen llevar consigo (teléfono móvil, smartphone, tablet, pc, ipod, etc.).
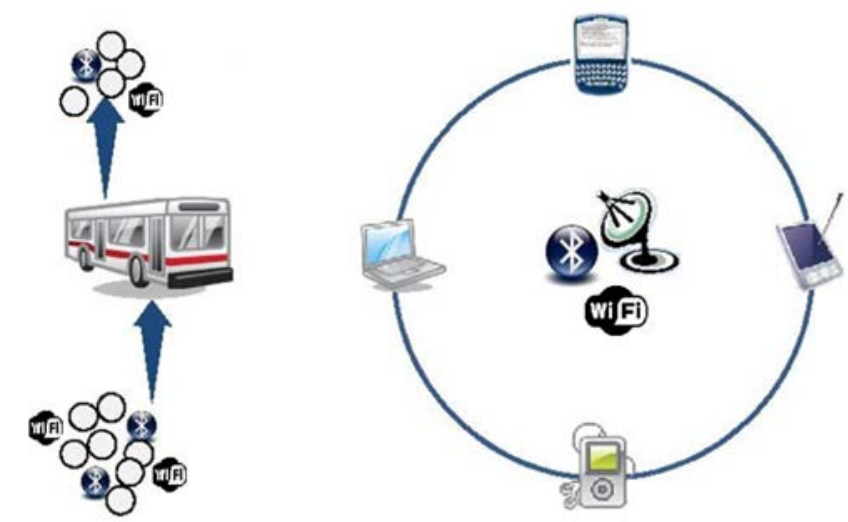

Figura 2. Gráfico conceptual

Se espera que con esta información, el sistema permita a las operadoras realizar una gestión más eficiente de los recursos y mejorar el nivel de servicio al cliente. Como ya se ha introducido, entre las posibles aplicaciones y usos del sistema se encuentran:

- Planificación: estimación de la matriz de trayectos origen-destino (O-D) de una línea de autobús, a partir de las muestras generadas por las detecciones de los dispositivos activados

- Gestión/regulación: 
o estimación de la carga actual del autobús a partir de la información suministrada en tiempo real de las validaciones acumuladas, el número de pasajeros con dispositivo detectable que suben y bajan en cada parada (hasta la actual), y la estimación histórica de la matriz O-D.

o previsión de la carga de pasaje en paradas subsiguientes a partir de la estimación de la carga "actual" y la estimación histórica de la matriz O-D.

- Otros: estimación del nivel (porcentaje) de fraude, segmentación por perfil de usuario para la mejor adecuación/idoneidad publicitaria, etc.

Para validar el uso de esta técnica, es necesario calibrar el nivel de ajuste o error, estudiar la viabilidad de cálculo de la matriz de trayectos (matriz O- D) a partir de la muestra generada por los pasajeros que viajan con su dispositivo wifi o bluetooth activo, y valorar su capacidad de estimación de la carga en tiempo real. Esta validación se llevará a cabo a través de unos parámetros de decisión y un estudio que permitirá tener en cuenta las variables y condicionantes que podrían existir para cada línea de autobús, así como los condicionantes externos (meteorológicos, día de la semana, hora, etc.). Además, se evalúa la posibilidad de hacer previsiones de la carga del autobús en tiempo real.

El alcance de la tesis pretende la validación del sistema propuesto sin perder de vista el concepto de producto dado su carácter innovador, mediante la construcción de un software procesador de datos, el desarrollo de la tecnología necesaria, la validación experimental con pruebas piloto y la construcción del sistema como producto acabado.

El sistema permitirá a las empresas de transporte ser capaces de determinar, en tiempo real y futuro, el nivel de ocupación de todos los vehículos entre paradas, sin desvincular el origen del destino. 

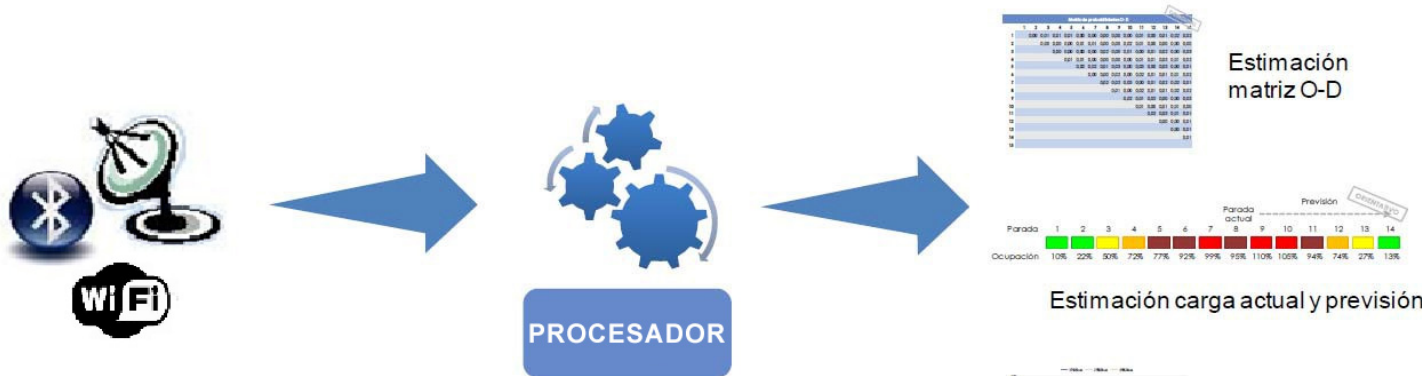

Estimación carga actual y previsión

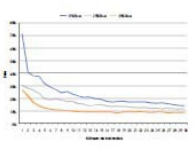

Errores de estimación

Figura 3. Cadena de valor

\subsubsection{Antecedentes}

La normas europeas de regulación en materia de calidad del servicio prestado al viajero y, consecuentemente, las distintas compañías de transporte que operan dicho servicio, establecen los ratios máximos en el grado de ocupación de los vehículos que configuran los compromisos de calidad (de 3 a 4,5 pasajeros $/ \mathrm{m}^{2}$; control de autobuses completos consecutivos, etc.). Asimismo, los nuevos Sistemas de Ayuda a la Explotación (SAE) de 3a Generación, son los que se están dotando los operadores de transporte, incluyen o deberían incluir, entre otras prestaciones:

- Algoritmos de regulación avanzados y parametrizables

- Localización automática y continua

- Paneles de Información al Usuario en paradas e interior de Autobuses

- Niveles de ocupación del Autobús

- Sistemas Avanzados de Información al Usuario

Así, el problema del conteo automático de pasajeros viene siendo abordado, desde hace tiempo, por los distintos departamentos de Planificación del Servicio, de Regulación, de Validación y Venta o de Telecomunicaciones en muy diversas compañías de autobuses, metro, tranvía y ferrocarril. En definitiva no es posible regular sin conocer el estado de carga de los vehículos; no se pueden planificar las frecuencias de paso sin disponer de los patrones de uso, ni configurar el mapa de líneas si se carece de la información pareja sobre el origen y destino de los trayectos más habituales; por último, disponer de todos estos datos 
requiere del concurso de tecnologías varias en el ámbito de la información y las telecomunicaciones (TIC).

\subsubsection{Justificación de la necesidad del trabajo de tesis como solución al problema planteado}

Para seguir garantizando cotas altas de calidad de servicio al ciudadano, se considera interesante y conveniente la incorporación de sistemas e instalaciones de conteo global que, desde la perspectiva del usuario, supongan una mejora en la gestión del autobús urbano y faciliten el uso del mismo, a la vez que permitan el análisis de la información para su estudio con el fin de obtener una mejora continua del servicio y explotación. Con esta perspectiva se consigue:

- Mejorar la calidad del servicio tanto en la explotación en tiempo real como en futuro, gracias a la información disponible para su análisis.

- Contribuir en la detección de incidencias y disminuir su número y el tiempo de resolución de las mismas, ya que supone la incorporación de nuevos elementos de control.

- Publicar información en tiempo real del estado y disponibilidad de todos y cada uno de los itinerarios de autobús para que el usuario del mismo pueda en cada momento tomar la mejor de las alternativas disponibles en función de sus necesidades.

- Agilizar y coordinar la toma de decisiones, actuando como mecanismo de engrase para los demás sistemas de monitorización que permitan disponer de la mayor cantidad de información disponible en cada momento en lo que se refiere a la movilidad y el transporte colectivo.

El sistema como ya se ha comentado, permite a las entidades gestoras de los servicios de transporte público la mejor planificación y gestión diaria de su red. A nivel de planificación, permite adecuar los servicios de transporte público a la demanda. Con la estimación de la matriz de trayectos origen-destino, y dada su fiabilidad, se pueden plantear reestructuraciones de líneas para satisfacer la demanda de los usuarios. En cuanto a la gestión y regulación, permite ofrecer información online de los niveles de ocupación de los autobuses y de los niveles esperados/previstos para las paradas subsiguientes. Así, las entidades gestoras 
podrían enviar refuerzos a una parada determinada a partir de la cual la previsión de carga estuviera por encima de la capacidad del autobús.

Este planteamiento precisa de una plataforma tecnológica que ayude a gestionar y supervisar, en tiempo real y futuro, la explotación de las redes de transporte, desde el punto de vista de los tres criterios básicos de la misma: tráfico, seguridad y mantenimiento. Para garantizar el cumplimiento de estos criterios de explotación, es clave disponer de información sobre el uso de la red que permita, mediante un cuadro de mando, monitorizar el acercamiento de la realidad a los objetivos de operación y mantenimiento.

En la actualidad, sólo se dispone de una parte de la información de conteo de pasajeros necesaria, concretamente la que se pueda operar a través del sistema de validación (billetaje). Aun tratándose de un sistema de muy altas prestaciones está diseñado para el control y supervisión del acceso a bordo del pasaje sin contemplar su relación con el conteo de bajadas, por lo que es insuficiente para determinar el número de pasajeros a bordo. Por consiguiente, parece clara la necesidad de disponer de una plataforma tecnológica que englobe ambos conteos, el de subida y el de bajada, mantenga la vinculación entre el origen y el destino del pasaje (la matriz OD), y permita el intercambio automático de información con los demás sistemas locales existentes (ya sea esta información referente a explotación, mantenimiento o seguridad) con vistas a impedir la degradación del servicio prestado por el transporte colectivo (como consecuencia de una menor eficiencia en la explotación del día a día o en la gestión y resolución de incidencias).

El factor innovador del trabajo de tesis consiste fundamentalmente en la potencia informativa del sistema desarrollado, la utilización de tecnología inalámbrica para la detección (a bordo o en dársena) de los dispositivos personales, y la definición de los métodos de estimación/extrapolación a partir de la muestra. La solución propuesta aprovecha el porcentaje cada vez mayor de pasajeros que suele llevar consigo algún dispositivo equipado con wifi o bluetooth detectable mediante un dispositivo compatible con dichos estándares inalámbricos operando a la misma frecuencia de trabajo. De esta manera se extrae una muestra del censo. El sistema no realiza un escrutinio 
(universo) si no una encuesta (muestra, es decir, un dato heurístico que puede hacerse plausible tras su calibrado posterior.

Con la utilización conjunta de ambos estándares se pretende abundar en éste propósito, aprovechando la "segmentación" que se observa en el mercado de dispositivos móviles, dónde la mayoría de los smartphones, provistos de conectividad wifi, incorporan una release bluetooth con apagado automático (pasa a modo no visible IOS y android). Y, viceversa, los dispositivos con bluetooth always on carecen de wifi (No es cierto, por ejemplo las blackberry (6,9 \% cuota 2012) permite ambos y symbiam también 6\% cuota mercado 2012) (¿Qué ocurre con los terminales que llevan ambas interfaces activas? ¿Cómo sabemos que es el mismo dispositivo?). Es decir, la utilización conjunta de ambos estándares garantiza una mayor uniformidad en la distribución de dispositivos entre la población, fortaleciéndose por tanto la validez estadística del proceso y la capacidad predictiva de la matriz OD así obtenida. En el momento de redactar este trabajo de tesis (2015) ya existen releases BT basadas en el nuevo estándar 3.0 o Low Energy, que eliminan el problema descrito anteriormente.

Para validar este sistema se requiere la realización de un test del que poder obtener los márgenes de error esperados en cada caso, utilizando para ello un modelo del comportamiento real. La precisión de la estimación dependerá de la proporción del número de dispositivos en relación al número real de pasajeros y del número total que hay en cada autobús en un momento dado. Se trata de obtener, en definitiva, la "tabla de fiabilidad" del sistema, que relaciona el grado de exactitud en la estimación de conteo, es decir, cómo fluctúa el margen de error en la estimación de la cuenta en función del porcentaje de dispositivos detectados. En definitiva, esta relación fijará un valor umbral en el porcentaje mínimo de dispositivos presentes necesarios (el "índice de penetración" con wifi o bluetooth), lo que puede redundar en posteriores recomendaciones para el incremento de la fiabilidad.

Para la implementación de la prueba, además del validado del sistema, se requiere el desarrollo de las componentes hardware y software con los que obtener los datos de conteo reales, transmitirlos y procesarlos. 
La automatización del conteo que aquí se propone, pretende reemplazar al tradicional equipo humano dedicado esporádicamente al conteo manual (uno o dos encuestadores apostados en las puertas de los vehículos o en las salidas de las dársenas o andenes) que, necesariamente, sólo cubre una muestra de las líneas, del calendario o de las franjas horarias. Pero la bondad del sistema aquí propuesto, su verdadero núcleo, no sólo se hallaría en éste aumento de eficiencia - el siempre deseable hacer lo mismo por menos- si no en la inteligencia añadida a la gestión de esos recursos - la filosofía smart city del más por menos-, al obtenerse unas matrices OD de capacidad predictiva mucho mayor. Es, en la comparación de estas distintas potencias predictivas, donde se establece el grado de exactitud (el nivel de error, si se quiere decir así) de ambos métodos, manual o automático.

\subsubsection{Descripción en relación al problema planteado del estado de la tecnología en España y en el extranjero, y como el sistema propuesto va más allá del estado del arte.}

En este intento de sustitución de la encuesta manual, se han introducido con éxito desigual algunos sensores y dispositivos no intrusivos, con el objetivo en algunos casos de contar el pasaje que sube y baja, y en otros de obtener directamente la carga a partir del peso soportado por el autobús.

Las opciones tecnológicas desarrolladas hasta el momento son:

- La instalación de alfombras sensibles a la presión en las entradas y salidas de los autobuses

- El uso de microbarómetros, instalados en el interior de los neumáticos o en los sistemas de suspensión.

- El uso de sensores por haz de luz infrarroja.

- La vigilancia por video. Reconocimiento de imágenes.

- La instalación de sensores térmicos

Además, las tecnologías anteriores están limitadas al control del flujo, y en ningún caso capturan información origen-destino. Estas tecnologías son adecuadas cuando se trata de conocer la afluencia o aumentar la seguridad en tiendas, centros comerciales, museos, terminales aéreas, de autobuses o trenes.

El análisis que determina la inviabilidad de las opciones anteriores ha sido realizado previamente, confirmándose en todas ellas su imprecisión y/o elevado 
coste. En algunos casos, el nivel de exactitud siquiera es una variable dependiente de la inversión en equipos.

La conclusión común a todas estos "ensayos" establece que la realización del conteo no consiste tan sólo en la dotación de determinados dispositivos contadores como en la prestación de un servicio de conteo que implemente un sistema capaz de:

- Calibrar unos equipos hardware encargados de la recolección de datos.

- Procesar estos datos mediante el software adecuado a la finalidad del conteo.

- Mantener inmune el funcionamiento del sistema en un entorno cambiante.

El elemento diferencial de la presente propuesta respecto a las opciones ya desarrolladas radica en la información origen-destino que genera, muy valiosa cuando se quiere conocer la movilidad asociada a una línea de autobús, o hacer una previsión de su nivel de ocupación en un momento dado. El rastreo de dispositivos wifi o bluetooth (de la mac address de los mismos) ofrece la información del trayecto de cada pasajero detectado.

Con este trabajo de tesis se incorpora una nueva tecnología a la lista de alternativas posibles en sistemas de conteo automático de pasajeros, con el convencimiento de que dicha innovación proporciona información más fiable y completa, y supondrá en las compañías que lo pongan en práctica un valor actual neto de la inversión mucho menor que la requerida por los otros sistemas ensayados.

\subsubsection{Resumen técnico de las principales actividades del trabajo de tesis}

A continuación se presenta un resumen del enfoque fundamental del trabajo de tesis en sus dos bloques constituyentes principales: (A) el subsistema de adquisición, filtrado y transmisión (en adelante, AFT), y (B) el subsistema procesador que incluye extracción, conteo y predicción.

\subsubsection{Adquisición, filtrado y transmisión}


Determinar el nivel de ocupación de los autobuses y, en el mismo proceso, vincular el origen del pasaje con su destino, requiere detectar e identificar la ubicación de los pasajeros en determinadas situaciones (al subir al autobús, al bajar o al permanecer a bordo), "leyendo la identificación" y relacionando dicha lectura con la localización exacta de cada autobús en su recorrido. Se podría decir que, para realizar esta lectura es necesario "etiquetar" (con un tag, elemento asociado biunívocamente al pasajero que se va a contar). Evidentemente, tanto la etiquetación como la lectura se realizan de forma totalmente anónima, transparente y ajena para al usuario. Los tags permiten "identificar" al pasaje cuando sube o baja del autobús (cuando "permanece en" o "desaparece del" ámbito del lector). Así, la lectura de cada tag no solo es útil para su contabilización sino que también procesa la identificación del mismo y la relaciona con los otros parámetros significativos del autobús (parada, línea, itinerario...) y del evento (marcas de fecha, hora y tipo: subida o bajada). Por consiguiente, la gestión de datos explota esta terna: cifras de conteo, marcas de fecha y hora, y coordenadas de geoposición.

El sistema desarrollado aprovecha a modo de tag el dispositivo provisto de conexión wifi o bluetooth que muchos pasajeros llevan consigo. De esta manera, la detección de tales dispositivos se utiliza para estimar el número de pasajeros que hay en todo momento a bordo, cuando han subido o donde bajan.

En el proceso de adquisición y transmisión de los datos que permitan esta estimación se distinguen, pues, tres partes claramente diferenciadas: la detección de dispositivos propiamente dicha, el procesado de los datos obtenidos resultantes de esta detección y, por último, la transmisión al host de una tabla de los datos procesados.

\subsection{Detección de dispositivos y adquisición de datos}

Un lector a bordo del autobús realiza lecturas periódicas de los dispositivos emisores de radiofrecuencia dentro de la zona de cobertura de su antena. Estas lecturas no tienen por qué coincidir en su totalidad con la muestra deseada: la de los pasajeros embarcados. Concretamente, la antena puede captar dispositivos en vehículos o peatones próximos. Este problema se subsana con el procesado posterior de los datos. Los parámetros configurables en esta etapa son la periodicidad de las lecturas y el posicionamiento y directividad de las antenas. Es 
interesante analizar la conveniencia de intensificar el número de lecturas o escaneos en las proximidades de las paradas, cuando la variabilidad de los pasajeros es mayor.

Se abordó esta fase del trabajo de tesis secuenciando los dos estándares de comunicación, bluetooth y wifi. Ambos operan a la misma frecuencia de transmisión, utilizan protocolos similares y conllevan la identificación del dispositivo emisor. Sin embargo, en la tecnología bluetooth coinciden dos factores cuya consideración la hicieron tecnología candidata para el arranque: su scanner reviste mayor sencillez tecnológica y su “índice de penetración” entonces, era bastante superior al wifi. Así, empezando el desarrollo por la aplicación de detección de los bluetooth, estábamos en condiciones de abordar el módulo wifi en un tiempo menor, y disponiendo de unos primeros resultados de operativa que permitían arrancar en paralelo las siguientes etapas del desarrollo.

\subsection{Filtrado de datos}

La finalidad de esta etapa es determinar los dispositivos que hay a bordo del vehículo entre paradas consecutivas. Para conseguir esto se utiliza un filtrado temporal de los datos obtenidos en la etapa anterior. Es decir, se tomarán como dispositivos a bordo, aquellos dispositivos que hayan sido detectados en repetidas lecturas no necesariamente sucesivas en el período comprendido entre dos paradas. Para ello es esencial disponer de un mecanismo de identificación de parada (SAE, apertura de puertas, GPS...). Identificada ésta, la tabla con la lista de dispositivos a bordo queda unívocamente asignada a la parada que le corresponde, asignación de la que resultan las combinaciones origen-destino, el elemento diferenciador de esta propuesta.

\subsection{Transmisión al host}

Una vez obtenidos los datos en la etapa anterior se han de transmitir a un host remoto para su tratamiento posterior, tal como se explica más adelante. Esta transmisión se realizará vía GPRS ó UMTS, después de cada parada, de manera que el sistema trabaje en tiempo real. 0 bien mediante un protocolo de saltos que además aporta servicios de valor añadido tanto a la compañía de transporte como a los pasajeros. Este protocolo se explica en el capítulo 4 de este documento de tesis. 


\subsubsection{Simulación, conteo y predicción}

Como enfoque preliminar del sistema desarrollado, se presentan a continuación los métodos de previsión y los resultados que se esperan obtener: la caracterización de las líneas de autobús, la metodología de la simulación, la estimación de la matriz 0-D, la estimación de la carga en tiempo real y la previsión de la carga.

\subsection{Caracterización de una línea}

Una parte fundamental de este estudio, del que dependen la calidad, el grado de representatividad de la realidad de las simulaciones, y por lo tanto todos los resultados que se obtendrán es la definición de una línea.

A continuación, se muestra una lista preliminar de elementos caracterizadores para una línea que se deben incorporar.

- Tipo de día: laboral o festivo, o incluso diferenciar cada día de la semana

- Hora o intervalo horario

- Meteorología/Previsión meteorológica: lluvia, etc.

- Características de la línea de transporte público:

o número de la línea

o número de paradas

o subidas y bajadas medias por parada

o capacidad del recurso (autobús) asignado

o porcentaje de trayectos de movilidad obligada

o probabilidad de que un pasajero lleve un dispositivo detectable

\subsection{Simulación de una línea de autobús a partir de la generación estocástica de pasajeros}

Dado un conjunto de parámetros caracterizadores de una línea (en concordancia con lo descrito anteriormente), se generará un conjunto de líneas de dichas características.

Los autobuses serán simulados mediante la generación estocástica de pasajeros. Dados el número de paradas, el número medio de subidas y bajadas en cada una y la capacidad del autobús, se generará un conjunto de pasajeros que conformará una instancia de ese autobús. 


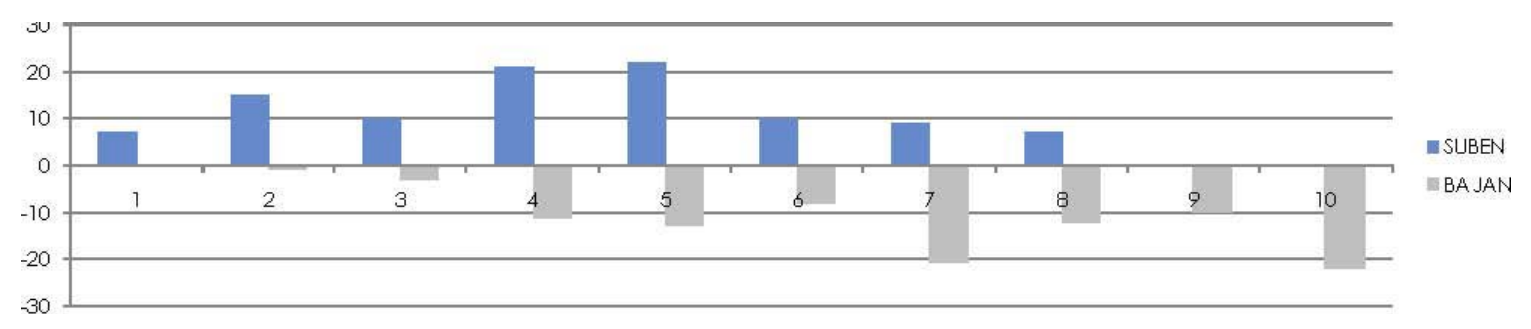

Figura 4. Histograma de subidas y bajadas de un autobús con un recorrido de 10 paradas

Para que la simulación sea lo más fiel posible a la realidad es necesaria la introducción de variabilidad (parámetros estocásticos) en el procedimiento. Por ejemplo, para el número de pasajeros que suben y bajan en cada parada, un trabajo de campo permitirá ajustar una o varias distribuciones empíricas.

Para introducir el porcentaje de pasajeros con dispositivo detectable se deberá decidir aleatoriamente (según la probabilidad) si un pasajero lleva o no activado el wifi y/o el bluetooth de su dispositivo.

Esta variabilidad que se introduce en el modelo, tiene que ser lo más fiel posible a la realidad, dado que de ella dependen directamente los resultados que se obtienen de la simulación.

Otro concepto que se debe incluir en el modelo es la movilidad obligada. Con una cierta probabilidad habrá trayectos (parejas origen-destino) de movilidad obligada, que se repetirán, por ejemplo, de lunes a viernes (pasajeros que sólo disponen de ese medio y en ese horario para desplazarse). Esta característica es especialmente crítica para nuestro análisis. La movilidad obligada hace que nuestras observaciones sean repetitivas y no aporten información nueva, y por eso puede establecer barreras o umbrales de error que no podamos mejorar. Dicho de otra manera, las sucesivas muestras no aportarán información nueva y no ayudarán a explicar el comportamiento de la población. Por tanto, la movilidad obligada produce muestras relacionadas o dependientes, pudiendo dar lugar a estimadores sesgados de la matriz 0-D y la carga en tiempo real del autobús. Con todo, cuanto más "urbana" es una línea, menor es su porcentaje de movilidad

\subsection{Estimación de la Matriz O-D - Planificación}

Uno de los resultados esperados de este trabajo de tesis es la obtención de la matriz de movilidad de las líneas. El aprendizaje de la matriz de probabilidades 0- 
D se realizará mediante la combinación de las instancias diarias de la matriz 0-D que generarán los pasajeros con un dispositivo activado y detectado.

\begin{tabular}{|c|c|c|c|c|c|c|c|c|c|c|c|c|c|c|c|}
\hline \multicolumn{16}{|c|}{ Matriz de probabilidades O-D } \\
\hline & 1 & 2 & 3 & 4 & 5 & 6 & 7 & 8 & 9 & 10 & 11 & 12 & 13 & 14 & 10 \\
\hline 1 & & 0,00 & 0,01 & 0,01 & 0,01 & 0,00 & 0,00 & 0,00 & 0,00 & 0,00 & 0,01 & 0,00 & 0,01 & 0,02 & 0,02 \\
\hline 2 & & & 0,00 & 0,00 & 0,00 & 0,01 & 0,01 & 0,00 & 0,00 & 0,02 & 0,01 & 0,00 & 0,00 & 0,00 & 0,00 \\
\hline 3 & & & & 0,00 & 0,00 & 0,00 & 0,00 & 0,02 & 0,00 & 0,01 & 0,00 & 0,01 & 0,02 & 0,00 & 0,03 \\
\hline 4 & & & & & 0,01 & 0,01 & 0,00 & 0,00 & 0,00 & 0,00 & 0,01 & 0,01 & 0,03 & 0,01 & 0,02 \\
\hline 5 & & & & & & 0,02 & 0,02 & 0,01 & 0,03 & 0,00 & 0,03 & 0,00 & 0,03 & 0,00 & 0,01 \\
\hline 6 & & & & & & & 0,00 & 0,00 & 0,02 & 0,00 & 0,02 & 0,01 & 0,01 & 0,01 & 0,02 \\
\hline 7 & & & & & & & & 0,02 & 0,02 & 0,03 & 0,00 & 0,01 & 0,02 & 0,02 & 0,01 \\
\hline 8 & & & & & & & & & 0,01 & 0,00 & 0,02 & 0,01 & 0,01 & 0,02 & 0,02 \\
\hline 9 & & & & & & & & & & 0,02 & 0,01 & 0,02 & 0,00 & 0,00 & 0,03 \\
\hline 10 & & & & & & & & & & & 0,01 & 0,00 & 0,01 & 0,01 & 0,00 \\
\hline 11 & & & & & & & & & & & & 0,02 & 0,03 & 0,01 & 0,01 \\
\hline 12 & & & & & & & & & & & & & 0,00 & 0,00 & 0,01 \\
\hline 13 & & & & & & & & & & & & & & 0,00 & 0,01 \\
\hline 14 & & & & & & & & & & & & & & & 0,01 \\
\hline 15 & & & & & & & & & & & & & & & \\
\hline
\end{tabular}

Tabla 1. Ejemplo de matriz de probabilidades origen-destino.

La matriz de probabilidades origen-destino, mapea la movilidad asociada a una línea y refleja la demanda de los usuarios. Con cada nueva instancia, se actualizará dicha matriz. El tamaño mínimo de la muestra (número de instancias históricas de la matriz 0-D generada por las detecciones de dispositivos que hay que acumular) para que el error de estimación esté por debajo de un cierto umbral se determinará mediante simulación. 


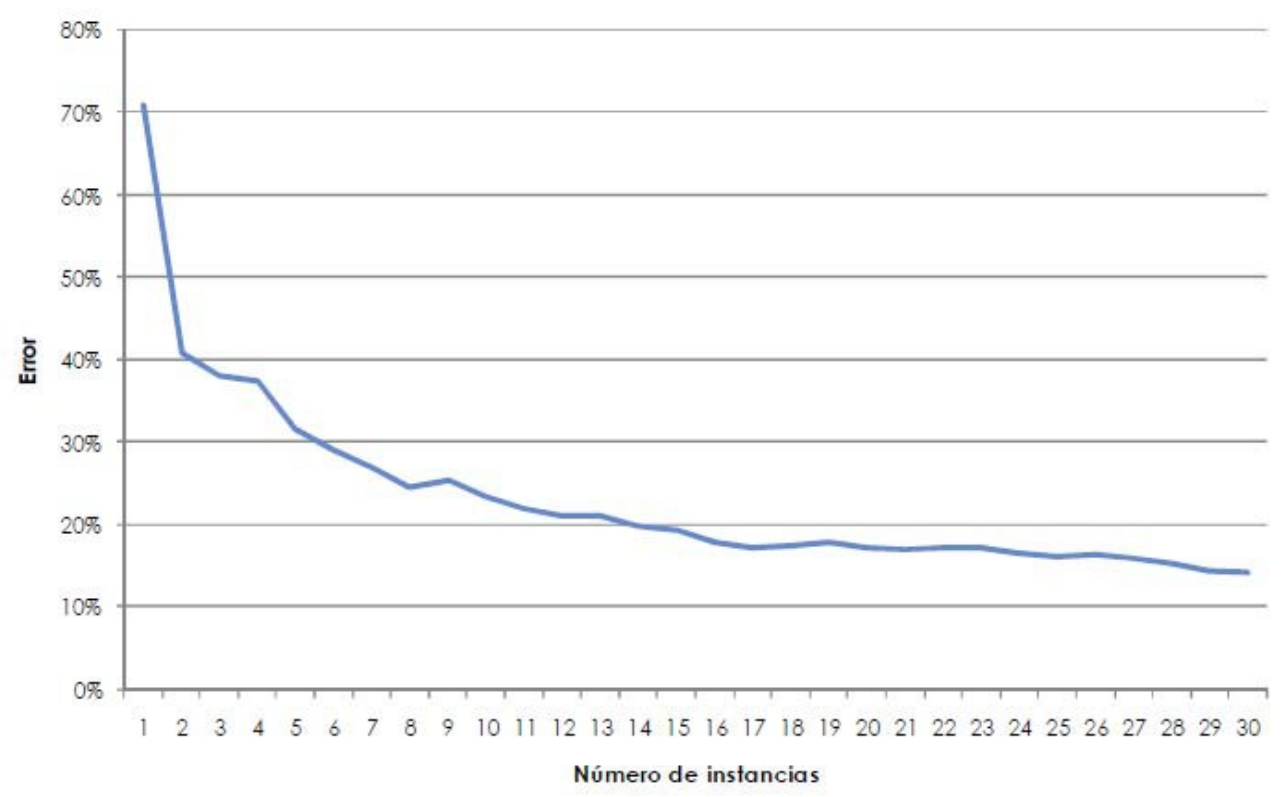

Figura 5. Impacto esperado del número de muestras de la matriz 0-D en el error de estimación

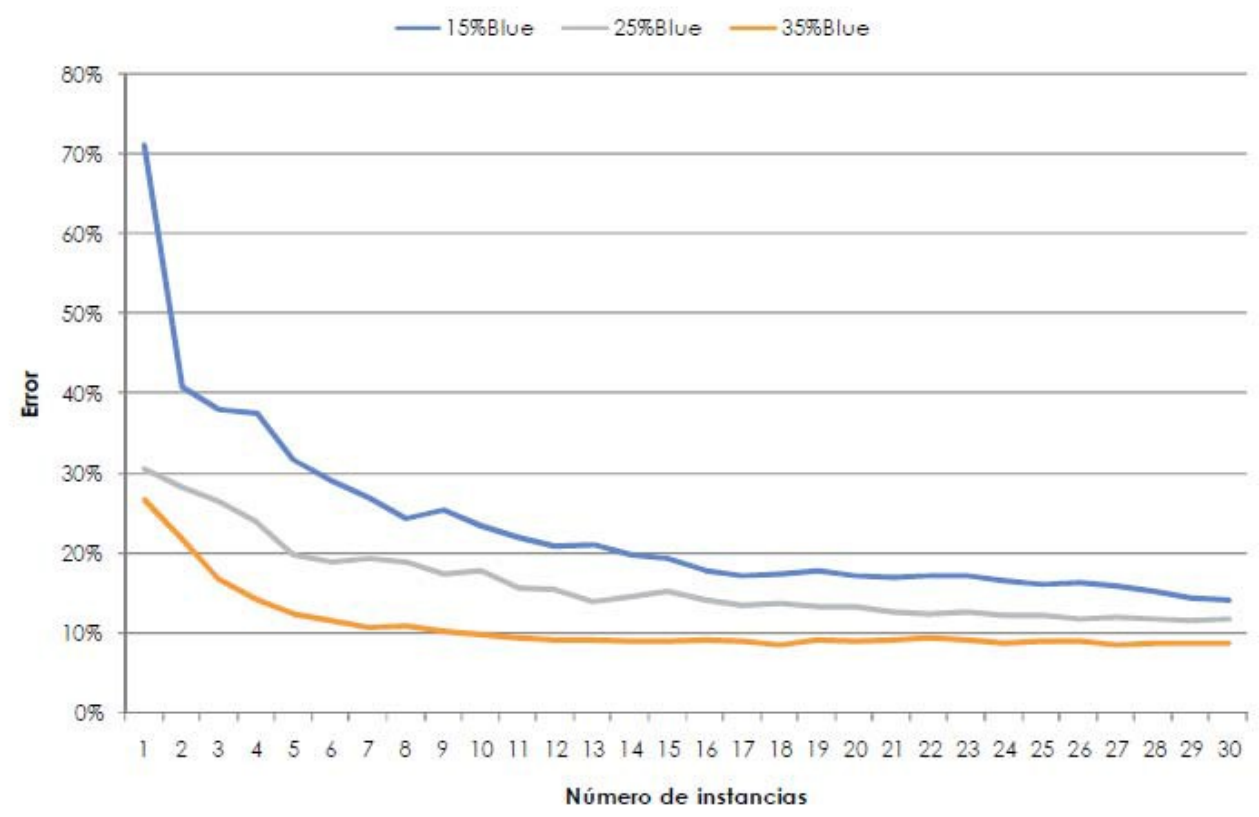

Figura 6. Impacto esperado del nivel de dispositivos detectados en el error de estimación de la matriz O-D

\subsection{Estimación de cargas On-line.}

A partir de las validaciones acumuladas en cada parada, las subidas y bajadas de pasajeros cuyo dispositivo es detectado y los datos históricos, se estimará la 
carga del autobús. Se deberán calibrar los parámetros de los pesos a la observación actual on- line y a los datos históricos.

Para asegurar la validez del resultado, se calculará con simulaciones el error de la estimación de carga para cada instancia (línea de las mismas características que la que se querrá estudiar en la realidad) de la batería de datos. Entonces se dará un valor medio del error y un intervalo o banda de confianza para dicho error.

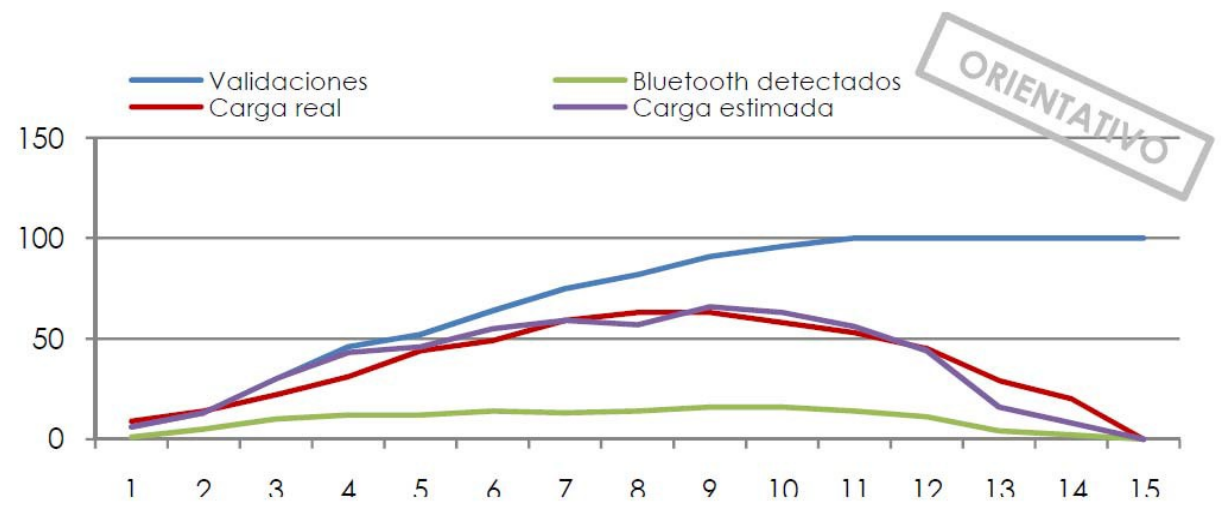

Figura 7. Ejemplo de estimación de la carga por tramos. Núm. Parada vs carga (número de pasajeros)

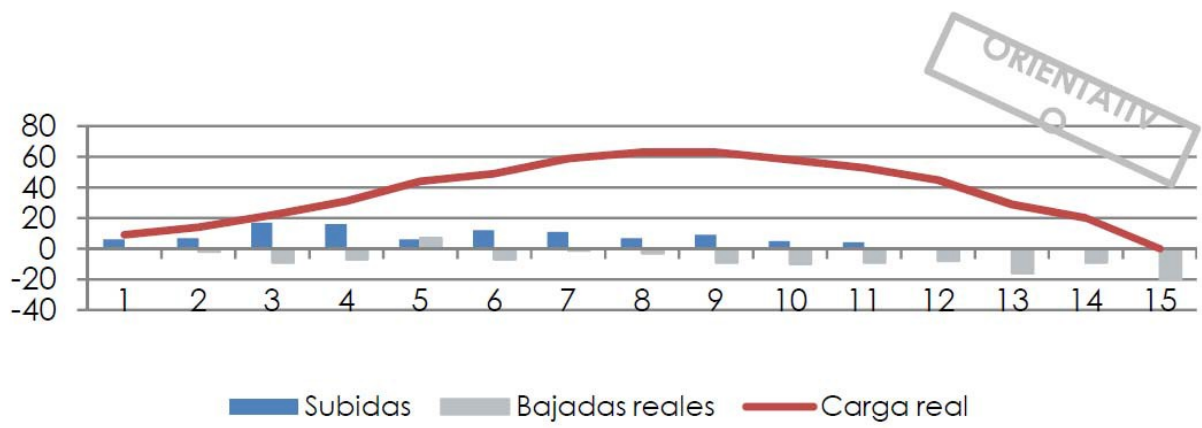

Figura 8. Subidas, bajadas y cargas reales en cada parada una línea.

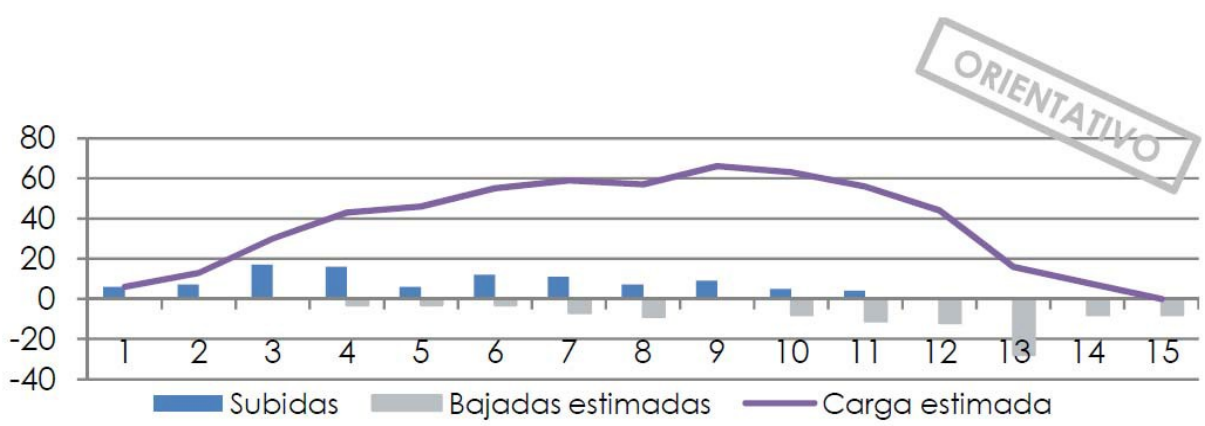

Figura 9. Subidas reales, y estimaciones de las bajadas y la carga. 
Mediante la estimación de cargas on-line se espera etiquetar en cualquier momento los autobuses de una red, según su nivel de ocupación (carga). Si se puede delimitar el error de estimación, se prevé factible estimar en qué intervalo de ocupación se encuentra la carga de un autobús en un momento dado. Se pintará el estado del autobús en un cierto tramo de un color en función de si la ocupación está entre el 0 y 25\% (verde), 25 y 50\% (amarillo), 50 y 75\% (naranja), 75 y 95\% (granate) o si es mayor que 95\% (rojo).

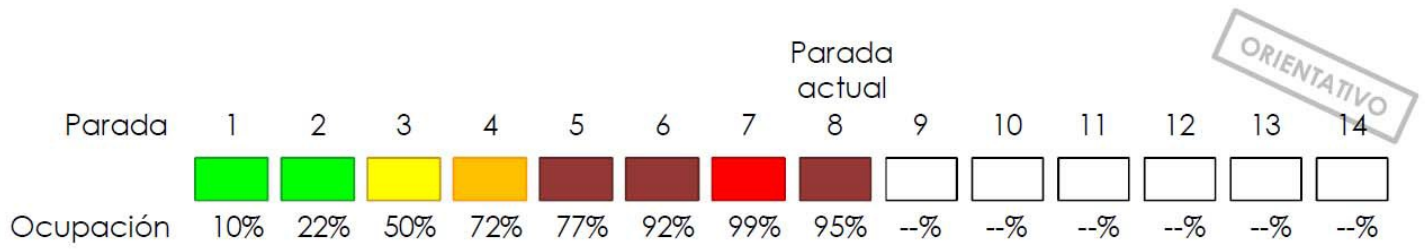

Figura 10. Estimación on-line de la carga

Se definirá más de una medida de error, para poder contemplar la estimación globalmente, pero también tener un control del error en los tramos de mayores cargas (mayor ocupación).

\subsection{Previsión de carga}

En caso de querer hacer una previsión de las cargas futuras, en tramos subsiguientes, se estudiará la posibilidad de hacerla a partir del histórico de cargas (estimación de la matriz 0-D) de la línea correspondiente.

Dada la estimación actual de la carga del autobús, se estudiará la posibilidad de hacer una previsión de la carga en los próximos tramos en base a la estimación histórica de la matriz O-D.

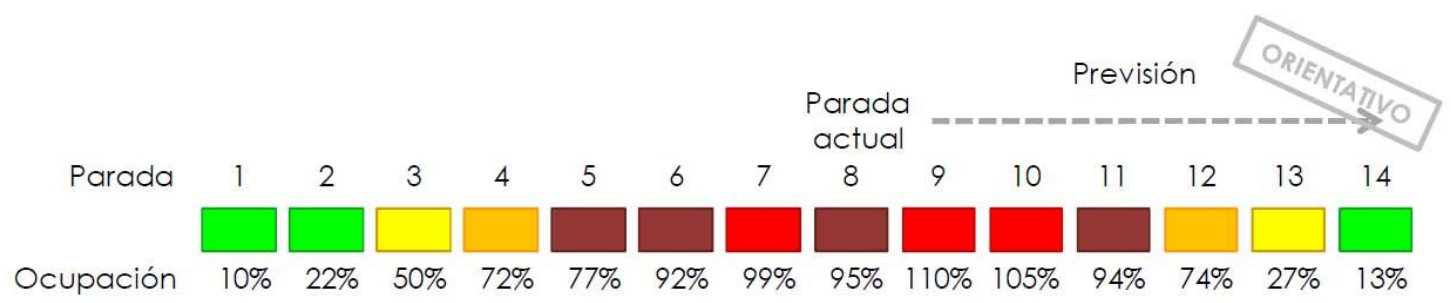

Figura 11. Estimación on-line de la carga y previsión para las próximas paradas 


\section{CAPÍTULO 2: Sistema de adquisición y filtrado.}

Continuando con la búsqueda de un sistema eficaz de localización en todos y cada uno de los espacios que el ser humano puede ocupar, es evidente la necesidad de diferenciar dos posibles entornos, los entornos cerrados y los entornos abiertos.

La localización en entornos abiertos con cobertura satelital está cubierta desde la aparición del Sistemas de Posicionamiento Global (GPS) [Ortiz'2003], al cual han convergido todas las tendencias existentes anteriormente.

El sistema GPS se basa en la utilización de varios satélites que proporcionan posiciones en tres dimensiones, velocidad y tiempo, las 24 horas del día, en cualquier parte del mundo y en todas las condiciones climáticas. Al no haber comunicación directa entre el usuario y los satélites, el GPS puede dar servicio a un número ilimitado de usuarios.

El mayor problema de esta tecnología se encuentra en la escasa sensibilidad a la hora de obtener señal procedente del satélite debido a la baja potencia de recepción. Lo que provoca la pérdida de cobertura cuando se obstruye la línea de visión directa (LOS). Esto supone una gran limitación en cuanto a utilizar el posicionamiento a partir de satélites en lugares donde no existe LOS entre ellos y el terminal de GPS.

De entre todas las propuestas de localización Indoor aportadas hasta hoy, no se ha llegado a una solución convergente y utilizable que dé cabida a una innovación tecnológica de relevancia tal y como supuso el GPS en entornos abiertos. Quizás pueda deberse a que ninguna de estas soluciones ha creado una necesidad en la sociedad debida a que produzca alguna ventaja o comodidad con respecto a no usar alguna de estas aplicaciones.

Este capítulo se centra en el estudio de un tema muy actual relacionado con la localización de personas por medio de su terminal móvil en emplazamientos cerrados, sin cobertura satelital, como aulas, departamentos, laboratorios, oficinas, servicios, cafeterías, etc. o abiertos como patios, jardines y otros. En definitiva se analizan los sistemas actuales más relevantes en lo que a localización en entornos Indoor se refiere, lugares en los que los GPS resultan ineficaces. 


\subsection{Estado del arte de los sistemas de localización en entornos Indoor}

Hasta el día de hoy, con la aparición nuevos estándares de comunicaciones inalámbricas, existen un elevado número de combinaciones entre protocolos y tecnologías que pueden utilizarse en interiores de edificios. Las más destacadas son Bluetooth, Wifi [IEEE802.11], RFID [Stephen'2008] y ZigBbee [IEEE802.15.4], en combinación con sistemas empotrados o redes de sensores.

\subsubsection{Soluciones con estándar Bluetooth}

Bluetooth es una tecnología diseñada para ofrecer conectividad a redes personales mediante un dispositivo móvil de forma económica. Permite conectar múltiples aparatos Bluetooth: ordenadores portátiles, PDAs, teléfonos móviles, etc., y ofrece conexión a una LAN o WAN a través de un punto de acceso. Bluetooth consigue un canal de comunicación de 721 kbps en un radio de acción de 10 metros, ampliable hasta 100 metros por medio de repetidores. La frecuencia que utiliza está entre 2,4 y 2,48 GHz, cuya gran ventaja es que es un rango de frecuencias abierto. Además, y debido a su concepción de tecnología móvil y económica, tiene un consumo de energía bajo. Para transmitir a una distancia de 10 metros, emplea $1 \mathrm{~mW}$ de potencia, mientras que para llegar a los 100 utiliza $100 \mathrm{~mW}$.

La aplicación práctica de esta tecnología es la posibilidad de montar redes inalámbricas en lugares donde haya dificultad para hacerlo de forma convencional, aunque hoy en día para este propósito se oferta otra tecnología, basada en los estándares del IEEE 802.11 que ofrece mayor ancho de banda y radio de conectividad. Por ello, Bluetooth se dirige más a la comunicación de dispositivos.

Existe un sistema de localización mediante Bluetooth [González'2002] [González'2003] para aplicaciones como comercio móvil y museos electrónicos, ambas de gran relevancia en el tema que nos ocupa. Básicamente, la idea es la ya mencionada en estas páginas: la implementación de servicios que requieren el conocimiento de la posición del usuario en tiempo real, para enviar información relativa del contexto al destinatario final.

El sistema, denominado Red de Localización Bluetooth [González'2002], transmite información de la posición del terminal móvil a los servidores, sin la participación del usuario. No es objeto de restricciones debido a la pérdida de línea de vista y funciona con dispositivos comerciales ya existentes (dispositivos con 
Bluetooth o terminales móviles de datos que admitan una tarjeta de expansión). El BLN está compuesto por pequeños nodos Bluetooth que establecen una topología de red espontánea con la inicialización del sistema. Puede coexistir con dispositivos Bluetooth que no son parte del sistema de localización, como impresoras y auriculares.

\subsubsection{Soluciones con estándar Wifi}

Hoy en día las tecnologías para red de área local (LAN) inalámbrica están implantadas en hoteles, cafés, aeropuertos y otros edificios en los que se considera rentable una oferta de acceso a Internet de alta velocidad. Estas nuevas infraestructuras también soportan localización de dispositivos móviles, por lo que las aplicaciones basadas en la posición para entornos de área local resultan viables.

En el ámbito del patrimonio y el turismo, se han puesto en funcionamiento redes de este tipo de forma experimental en museos (Metropolitan Museum de Nueva York, Tate Modern Museum de Londres...), excavaciones arqueológicas (Atapuerca, en combinación con Bluetooth), hoteles (Hotel Royal Sonesta de Boston), parques de temáticos (Disney World), obviamente con la idea de ofertar servicios basados en la filosofía de suministrar al usuario información de interés ligada a su posición.

Las redes inalámbricas cubren áreas de hasta 75 metros en el interior de edificios, y de 300 metros en el exterior, ampliables a varios kilómetros mediante antenas. Están implementadas en un grupo de estándares, conocido como 802.11 que comprende varias modalidades, cada una de ellas con unas características de ancho de banda y alcance determinadas. La modalidad más popular es la que se conoce como 802.11 b, introducida en 1997. Opera a una frecuencia de $2,4 \mathrm{GHz}$, y su índice de transferencia de datos es de $11 \mathrm{Mbps}$. Esta tasa se ve ampliamente superada por el estándar 802.11a, que llega a ofrecer 55Mbps en una frecuencia de funcionamiento de $5 \mathrm{GHz}$. El problema es que dicha parte del espectro no está disponible en países como España, Italia, Portugal o Alemania, pues es de uso restringido militar.

La localización mediante redes locales inalámbricas puede llevarse a cabo de diferentes maneras. La más sencilla es la basada únicamente en el punto de acceso más cercano al terminal. Este método confunde a menudo la planta del edificio, pues 
es fácil que la antena más cercana a un usuario ubicado en una determinada planta sea la misma que la correspondiente a un usuario situado en una planta superior, si la posición sobre el piso es similar. Por otra parte la señal es vulnerable debido a las interferencias, lo que puede afectar, además de a la precisión, a la seguridad de la comunicación.

Existe otra propuesta muy interesante [Ekahau'2015] basada en el almacenamiento de medida de potencia de señal en diferentes puntos del recinto cubierto. La técnica, conocida como WiFi mapping, arroja resultados más exactos que los métodos de triangulación celular, ofreciendo una precisión de 1 a 20 metros. Además, este sistema es sensible a los cambios de altura, es decir, reconoce fácilmente la planta del edificio en la que está el usuario.

\subsubsection{Soluciones con RFID}

La identificación por radiofrecuencia RFID [Stephen'2008] aporta alternativas y soluciones, factibles y viables, para el efectivo flujo de información, elemento estratégico en la actualidad. Representa una solución para el intercambio de datos a distancia. El RFID es una clara evidencia del alto nivel de avance de la microelectrónica, ingeniería de materiales, tecnología de radiofrecuencia aplicada y de la informática consistente en la miniaturización de dispositivos de radio que interactúan con el entorno.

Presenta una serie de aportes a todas las actividades de registro y transmisión de datos relativos a la identificación y especificaciones de artículos que físicamente puedan llevar un tag. Por ello, las aplicaciones no sólo se deben a la etiqueta o tag en sí, sino que se requieren dispositivos, codificación de datos, protocolos de información, comunicación y sistemas informáticos para lograr lo que se desea: intercambio eficaz y eficiente de información, telecomunicación y flexibilidad.

Comprendiendo lo anterior, para implantar un sistema RFID básico se necesita normas, hardware y software, tales como: detectores o lectores-ePc, capacidad de memoria adicional para datos complementarios, protocolos de comunicación y de anticolisión, y un sistema informático ad-hoc, como el SAVANT. 


\subsubsection{Soluciones con estándar ZigBee}

Existe un proyecto de sistema de localización mediante la tecnología ZigBee como aplicación de domótica [Lobillo'2008] cuyas fortalezas suponen una solución de ahorro energético y de aumento de confortabilidad en el entorno del hogar. Se fundamenta en un sistema de localización basado en las redes de sensores: el usuario interactúa con su hogar con su simple presencia. Un algoritmo estima la posición de la tarjeta coordinadora portada por el usuario respecto a unas balizas (motes) colocadas en posiciones conocidas.

La interfaz UPnP configura la red domótica en función de las medidas que la aplicación proporciona: posición, temperatura, humedad y luminosidad. Es una solución domótica de fácil instalación y bajo coste.

\subsection{Propuesta de un sistema concreto}

Como posible aplicación para la localización en entornos cubiertos puede estar la del control del personal en complejos de grandes empresas, por ejemplo, ya que se trata de buscar una solución que resulte interesante y de utilidad para la mejora en la calidad de gestión, control, supervisión y seguridad. Lógicamente luego se aplicará para el conteo de pasajeros en líneas de autobuses de transporte público.

En esta sección se detalla el estudio de las diferentes estrategias y dispositivos tecnológicos para la implementación de este sistema de seguimiento en emplazamientos divididos en zonas, así como la localización e identificación de personas a partir de la detección desde el punto de acceso multi-interfaz, y la gestión y presentación de información proporcionada por los dispositivos de localización.

Estas propuestas de aplicaciones de seguimiento tienen multitud de utilidades, entre las que destaca el aumento de la productividad de las empresas y sus empleados. Destacan estudios relacionados como [Lobillo'2008] [Guerrero'2007] [Murata'1993] [Ollikainen'2002] [Hii'2005] [Matthieu'2005].

\subsubsection{Descripción general del sistema}

La mayoría de los fabricantes de dispositivos Bluetooth (BT) [Ollikainen'2002] convencionales establecen como alcances máximos de estos dispositivos unas distancias comprendidas entre 10 y 20 metros en espacio libre, es decir, sin obstáculos que obstruyan la línea de visión directa. De modo que de existir 
alguno de éstos, como pueden ser paredes o puertas, el alcance podría verse reducido de forma considerable. Este aspecto negativo para otro tipo de utilidades puede resultar beneficioso en sistemas como el que aquí se propone, ya que ayuda a delimitar zonas más reducidas, como pequeñas habitaciones, laboratorios, departamentos, etc. Incluso zonas de paso que puedan resultar interesantes de controlar.

De este modo, una "estación" que lleve instalado un dispositivo BT y situado en una posición estratégica, que normalmente será el centro de la zona a controlar o una zona de paso, será capaz de detectar otros dispositivos BT que estén a su alcance. El problema surge con la detección de dispositivos en posibles zonas contiguas, puesto que es posible que no lleguen a superarse las pérdidas de propagación de la señal y sean detectados. Estos casos excepcionales se compensan reduciendo la potencia de transmisión en la estación, aunque no hay lugar a error debido a que la estación de la zona contigua también lo detectaría.

Las tecnologías comerciales que la gente adquiere junto con sus teléfonos móviles o PDAs dan la posibilidad de localizar personas y hacer su seguimiento de forma discreta. El principio de localización con el sistema que se implementa en esta tesis se basa en una de las debilidades de los sistemas Bluetooth, "el alcance".

La mayoría de los fabricantes de Bluetooth establecen como alcances máximos de estos dispositivos unas distancias comprendidas entre 10 y 20 metros en espacio libre, es decir, sin obstáculos que obstruyan la línea de visión directa. De modo que de existir alguno de éstos, como pueden ser paredes o puertas, el alcance se vería reducido de forma considerable.

De este modo, un dispositivo "estación" que lleve instalado un Bluetooth y situado en una posición estratégica que, normalmente será el centro de la habitación, será capaz de detectar otros dispositivos Bluetooth que estén a su alcance. El problema surge con la detección de dispositivos en las habitaciones contiguas, puesto que es posible que no lleguen a superarse las pérdidas de propagación de la señal y sean detectados. Estos casos excepcionales se compensan reduciendo la potencia de transmisión en la estación, aunque no hay lugar a error puesto que la estación de la habitación contigua también lo detectaría. Las estaciones implementadas en esta propuesta están formadas por un sistema 
embebido o empotrado que tiene conectado por USB un dispositivo Bluetooth y un interfaz WIFI.

A los empotrados, que funcionan bajo un Sistema Operativo Linux, se les han instalado inicialmente los drivers de ambos hardware y posteriormente se ha elaborado una aplicación en $\mathrm{C}$ que se encarga de realizar la búsqueda de dispositivos, reconocidos por detección de respuesta, solicitando información de identificación, como el nombre del terminal, dirección MAC, etc. El bucle, en cada detección, recoge la información que identifica el dispositivo y la envía por medio de un socket TCP por el interfaz WIFI a la dirección IP del ordenador que contendrá el programa de seguimiento y monitorización de todas las detecciones. De modo que se crean tantos sockets como dispositivos Bluetooth sean detectados.

El programa de seguimiento y monitorización es una aplicación Java en NetBeans IDE 6.1 que representa en una interfaz gráfica las detecciones de cada estación enlazadas con la estancia o emplazamiento correspondiente. Esta información es recibida por los distintos sockets de las distintas estaciones, con posibilidad de recibimiento simultáneo, ya que la gestión de los sockets se realiza de forma paralela con hilos de java.

Muchas de las tecnologías descritas en el estado del arte también son válidas para realizar la prouesta, puesto que todas son inalámbricas y proporcionan identificación de la situación del elemento físico que van con ellas al ser detectadas por una estación u otra. Sin embargo las principales razones de la elección de la tecnología Bluetooth para realizar el sistema de adquisición (búsqueda y localización de individuos) son:

- La inclusión de esta tecnología en terminales móviles y PDAs.

- Alcance ligeramente más elevado, en torno a 10 metros.

- Tecnología más implantada.

- Facilidad de adquisición.

- Discreción para un mejor seguimiento.

- No necesita insertar ningún elemento (tag) en algún objeto que lleve el individuo en cuestión. 
- Fácil gestión de la búsqueda y detección así como obtención de información desde un sistema embebido Linux que disponga de Bluetooth.

- Detección de forma rápida y efectiva.

\subsubsection{Arquitectura y estructura del sistema}

Las estaciones que cubren cada zona están compuestas por el conjunto de sistema embebido conectado por USB con un dispositivo BT convencional y un interfaz WIFI.

Un ordenador de monitorización que contendrá el programa principal en Java gestionará los sockets por medio de hilos concurrentes para recibir la información de las estaciones y la representará adecuadamente. La comunicación entre las estaciones y el ordenador de monitorización se realizará por medio de una red Ethernet, a través de los dispositivos WIFI de las estaciones y puntos de acceso como se muestra en la figura 12.

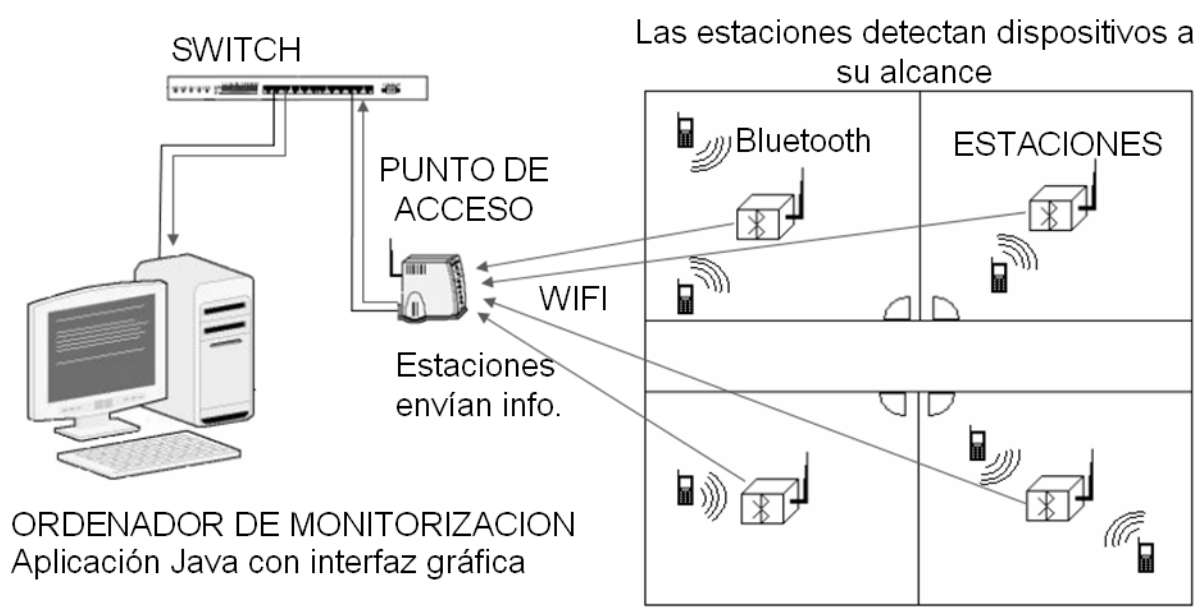

Figura 12. Estructura del sistema.

\subsubsection{Estaciones detectoras}

Las estaciones están distribuidas por todo el edificio situándose de forma única por estancia, zona o área de paso a controlar. De modo que interactuará con los dispositivos BT, detectándolos e identificándolos para comunicar al programa principal del ordenador de monitorización que un dispositivo con identificador y dirección MAC determinada ha sido detectado en la zona de detección correspondiente a la estación en cuestión. Esta información será transmitida por la red Wifi hasta el punto de acceso más cercano, que dirigirá los paquetes a la 
dirección IP establecida en los sockets de la aplicación de las estaciones, que se corresponde con el ordenador de monitorización.

Para detectar e identificar los dispositivos se utiliza la pila de protocolos de BT para Linux, BlueZ [Bluez'2015], que incluye las herramientas necesarias para detectar, conectar y obtener información básica de los dispositivos BT cercanos.

La aplicación de las estaciones es idéntica para todas y cada una de ellas. Está implementada en lenguaje $\mathrm{C}$ y realiza un escaneo de todos los dispositivos BT que están a su alcance por medio de solicitudes inquiry, a partir de la cuál se obtendrá una lista que contendrá las direcciones MAC de los dispositivos detectados. A continuación se recorre la lista analizando una a una todas las direcciones. Para cada una de ellas se solicita de forma opcional y con la instrucción hci_read_remote_name, el "nombre del dispositivo", y se crea un socket TCP para transmitir esta información al programa principal.

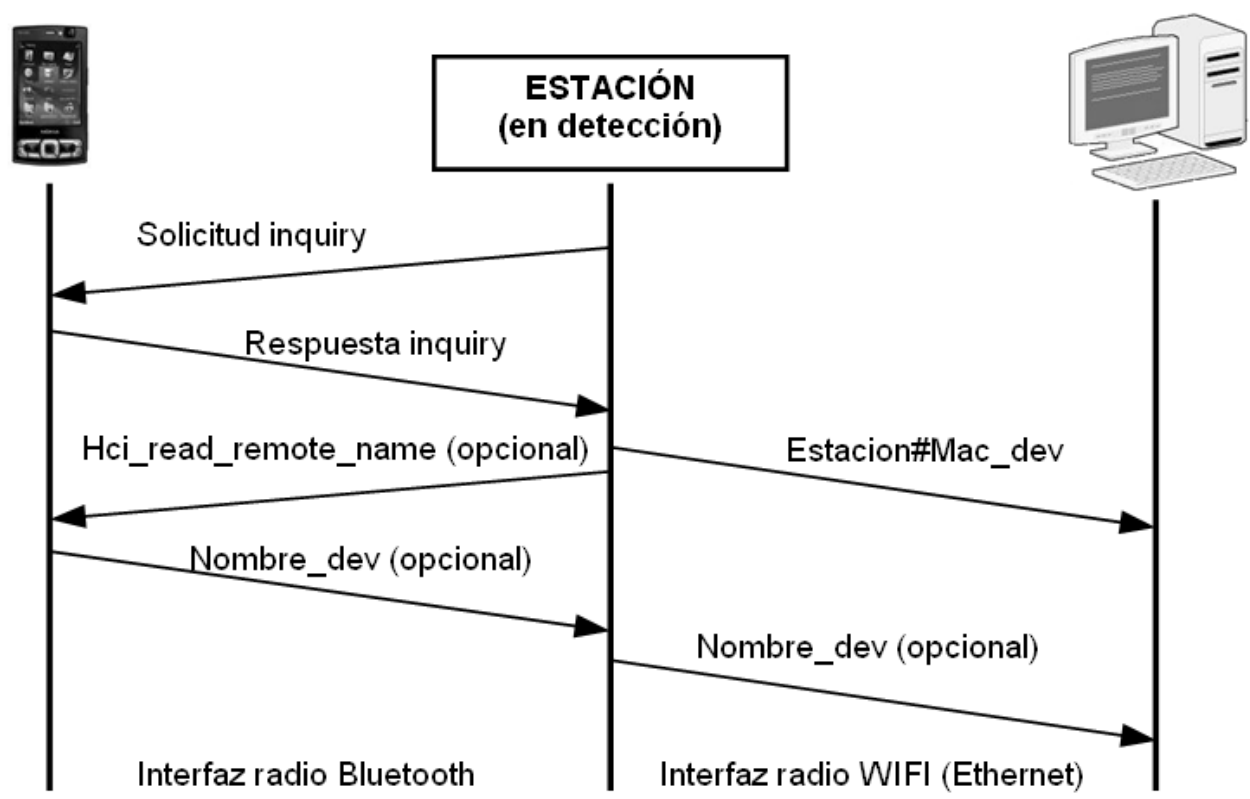

Figura 13. Diagrama de secuencia de una detección.

\subsubsection{Servidor de monitorización}

Es el equipo donde se encuentra en ejecución el programa de seguimiento y monitorización, aplicación Java que representa en una interfaz gráfica las detecciones de cada estación enlazadas con la zona o acceso correspondiente. Esta información es recibida por los distintos sockets que crean las distintas estaciones, 
con posibilidad de recepción simultáneo, ya que la gestión de los sockets en este programa de seguimiento se realiza de forma paralela con hilos de java [Pavon'2005]. Más adelante se muestra en detalle la programación y funcionamiento de esta aplicación, al igual que la de los empotrados.

\subsubsection{Sistema empotrado}

Existe un sistema embebido en el mercado que resulta óptimo para ejecutar esta aplicación de búsqueda de dispositivos en cada estación. Este sistema es la Foxboard LX832 [Fox'2015], que posee dos puertos USB para la conexión de las interfaces Wifi y Bluetooth, y un puerto Ethernet para el acceso al empotrado. El SDK (Software Development Kit) es totalmente abierto, y su entorno Linux permite ejecutar la aplicación. El procesador es AXIS ETRAX 100LX 100MIPS, su memoria flash es de 8 MB y la RAM de 32 MB. Ofrece la posibilidad de implementar y compilar el código en otro ordenador más potente y posteriormente trasladar el ejecutable a éste. Además es de tamaño muy reducido y tiene un coste muy bajo, condición necesaria para que el sistema resulte rentable, ya que se precisa de un empotrado por estación detectora. Se alimenta con una única fuente de alimentación de 5 Voltios consumiendo poco más de un vatio (280mA).

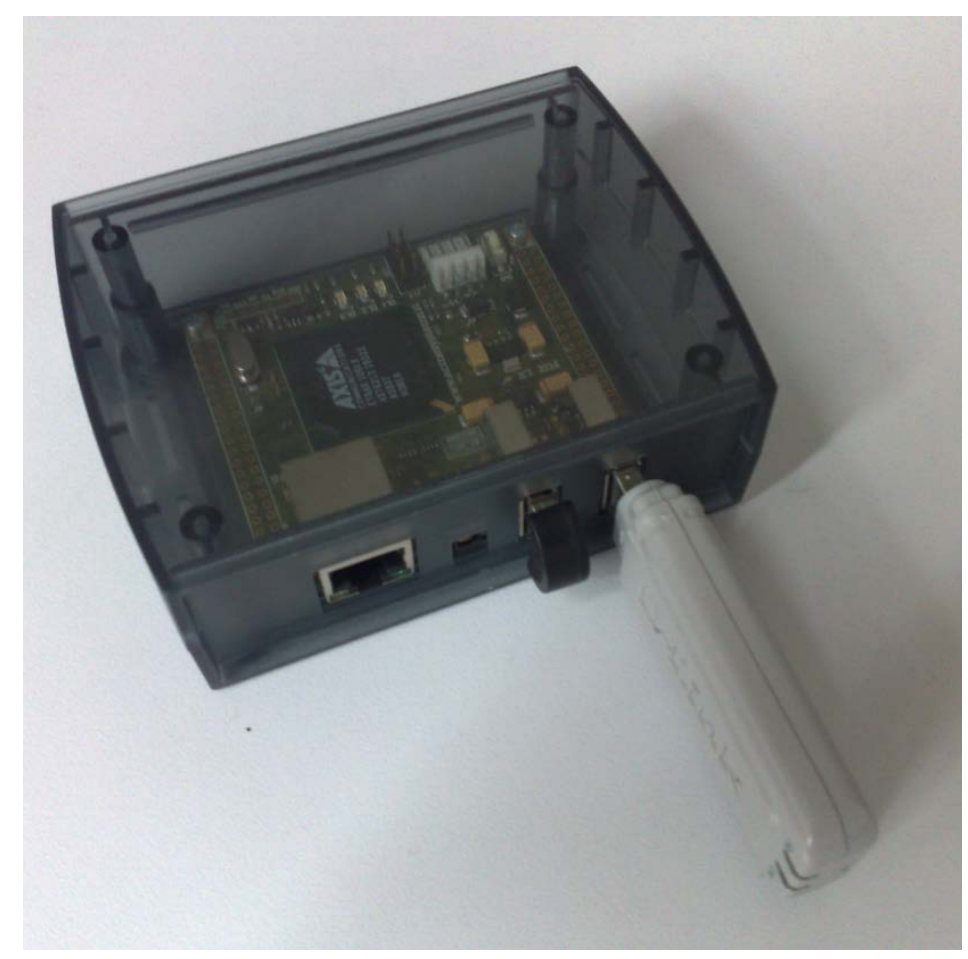

Figura 14. Estación formada por el sistema empotrado Foxboard LX832 y los periféricos USB de Bluetooth y Wifi. 
La placa permite extender otras conexiones como puertos serie, puertos paralelos, puertos IDE y otras líneas I/O, soldando los respectivos pines en las tiras J6, J7 y J10. En la figura 15 se pueden apreciar estas conexiones además de otros componentes como LEDs, puentes, un pulsador, etc.

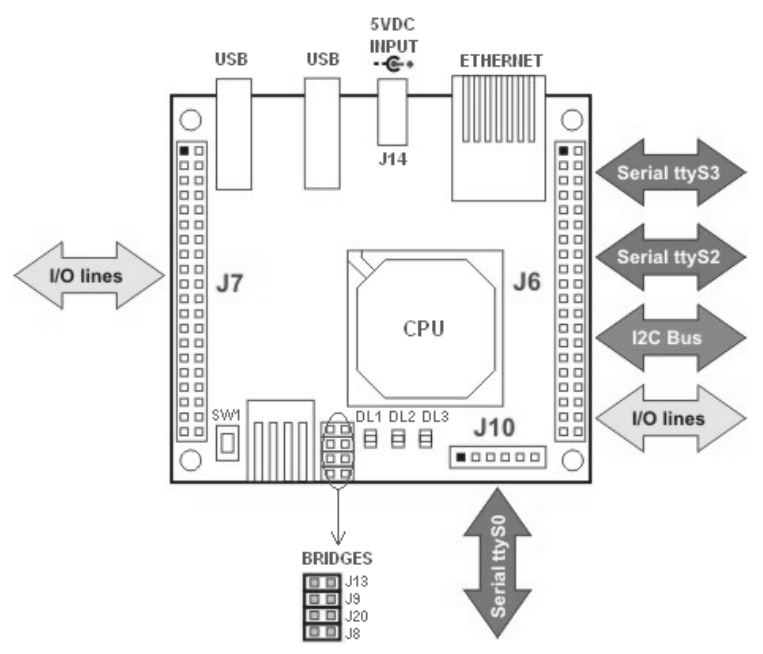

Figura 15. Diagrama de bloques del sistema Foxboard LX832.

- J14 es la entrada de alimentación de corriente continua de 5 Voltios.

- J10 es un puerto serie que se usa para la consola del sistema.

- $\quad S W 1$ es el botón de reset de la placa

- $\quad$ El diodo LED DL1, de color rojo, puede ser definida por el usuario para actuar con alguna aplicación software. En el arranque es usada por el Kernel para indicar un error, normalmente cuando no se establece una dirección MAC al adaptador Ethernet. Este error se explica más adelante.

- El DL2, de color amarillo, muestra el tráfico LAN sobre el interfaz Ethernet.

- El $D L 3$, de color verde, está conectado directamente a la entrada de alimentación, indicando el estado encendido de la placa.

- De los puentes, el J13, si se cierra, apaga la placa, y el $J 9$ la resetea. En arranque, si se cierra el $J 20$, se habilita carga de una imagen del sistema desde el puerto serie, y si se cierra el $J 8$ se habilita desde el puerto Ethernet.

\subsubsection{Programación de la aplicación de detección de las estaciones}

El código C que se implementa para la realización del escáner de dispositivos BT utiliza las librerías de BlueZ: bluetooth.h, hci.h y hci_lib.h. En ellas se describen 
principalmente algunas de las funciones y estructuras de datos que son necesarias para establecer la conexión entre las interfaces BT y para realizar la solicitud de identificación de cada uno de los dispositivos detectados.

Ésta es la parte principal de la aplicación, desde la cual, una vez obtenidos los identificadores (direcciones MAC), se llamará a otra función implementada en el mismo código que realizará el envío del identificador de estación junto con la MAC del dispositivo BT detectado.

\subsubsection{Detecciones BT}

En primer lugar es necesario realizar una reserva de memoria para almacenar en ella la lista de dispositivos detectados durante el proceso de inquiry. Esta lista estará formada por estructuras de datos que contendrán el identificador MAC de cada uno de los dispositivos BT que respondan a las solicitudes inquiry.

Las funciones que se utilizan para el establecimiento de la conexión, solicitud de identificación y extracción de la MAC de la estructura de datos son las siguientes:

- Hci_get_route(): Devuelve el identificador local de BT.

- Hci_open_dev(): Se usa para abrir el socket BT pasándole el identificador local obtenido con la función hci_get_route().

- Hci_inquiry(): Se encarga de realizar la solicitud inquiry y devuelve la lista con las estructuras de datos correspondientes a cada uno de los dispositivos que responden a la solicitud. Entre otros, posee un parámetro de entrada de tipo bandera que le permite habilitar la opción de limpiar la memoria caché antes de que realice la siguiente búsqueda de dispositivos.

- Ba2str(0: Extrae la dirección MAC de cada una de las estructuras de datos que forman la lista de dispositivos detectados.

Una vez que se obtiene la lista con la información que identifica a los dispositivos que han estado al alcance del BT de la estación, es necesario recorrer la lista con un bucle for para extraer las direcciones MAC una a una con la función ba2str(). De esta forma, en cada una de las iteraciones del bucle se obtendrá la dirección MAC que se concatenará al final de una cadena tipo char de 3 caracteres, de los cuales, los dos primeros identifican la estación en números decimales (de 1 a 
99) y el tercer carácter, '\#', se utiliza como separador, quedando una cadena de texto de la siguiente forma:

EE\#MM:MM:AA:AA:CC:CC

Por último, antes de que finalice la iteración, se realiza una llamada a la función clienteSocket() pasándole esta cadena de caracteres.

La diferencia de las aplicaciones pertenecientes a las distintas estaciones reside en los dos primeros caracteres (EE) de la cadena, ya que serán caracteres numéricos distintos para cada estación. De modo que todas las cadenas de texto enviadas por la aplicación de la estación 5, por ejemplo, serán:

05\#MAC_address_of_BT_phone_detected

\subsubsection{Socket TCP para envió de la información}

El envío de la cadena de texto descrita en el apartado anterior es realizada por la función clienteSocket (0 implementada en el mismo código C de la aplicación a continuación del main().

Esta función recibe como parámetro de entrada cadenas de texto de 20 caracteres de tamaño, y crea un socket TCP con el servidor implementado en el programa de monitorización. Una vez establecida la conexión, realiza el envío de la cadena de caracteres. Por último, cierra el socket una vez que la escritura en el socket finaliza de forma correcta.

Es válido utilizar cualquier número de puerto libre siempre y cuando coincida con el establecido en la clase Servidor.java que forma parte del programa principal de monitorización y que se describe en los siguientes apartados.

La dirección IP del servidor es siempre la del host donde corre dicho programa principal de seguimiento y monitorización. En los siguientes apartados se establece la 192.168.1.100.

\subsubsection{Aspectos críticos}

Los dispositivos que no están conectados se encuentran en estado stanby, realizando escuchas cada 1,28 segundos sobre 32 saltos de frecuencias. En este estado se encuentran los dispositivos BT (slave) a detectar [Helpy'2015]. 
Al pasar al estado inquiry, la estación actúa como master enviando mensajes en 16 saltos de frecuencias a las unidades slave. Si no hay respuesta retransmite en otros 16 saltos de frecuencia. Cuando las unidades slave responden desde sus direcciones MAC se establecen la conexiones y pasan a estado active. En este momento, ya se conoce la dirección MAC de los dispositivos BT al alcance de la estación.

La función hci_inquiry() posee algunos de los parámetros críticos para que el sistema funcione de forma correcta:

- Tiempo de inquiry: es necesario establecer un tiempo que permita descubrir todos los dispositivos al alcance. La especificación recomienda 10,24 segundos, pero con un tiempo tres veces superior al tiempo entre escuchas ( $3 \times 1.28$ segundos).

- Bandera IREQ_CACHE_FLUSH: Permite limpiar la caché antes de la siguiente búsqueda de dispositivos para que en una iteración no aparezcan dispositivos detectados en las anteriores iteraciones.

Por otro lado, notar que en la implementación del proceso de obtención de información del dispositivo BT detectado, no se obtiene el nombre del dispositivo (nombre_dev), ya que solo interesa obtener un dato que identifique con seguridad y de manera única el dispositivo detectado. No existe otro mejor que la dirección MAC, debido a que es única para cualquier dispositivo fabricado y se obtiene al establecer la conexión.

\subsubsection{Compilación de la aplicación}

Desde el mismo sistema Linux donde se implementa el código C de la aplicación es posible realizar su compilación.

El fabricante del empotrado facilita un Compilador WEB para subir el archivo de C, compilar el código fuente y obtener el ejecutable .out. Este compilador es válido para aplicaciones sencillas donde las librerías que se utilizan están incluidas en el SDK donde se ejecuta el compilador [ACM'2015]. No es el caso ya que esta aplicación hace uso de las librerías BlueZ. Por lo tanto es necesario tener instalado el Axis SDK en el PC Linux donde se va a compilar la aplicación con las librerías de Bluez también instaladas. 
Para realizar la compilación hay que acceder al directorio /home/fox/devorad-R_01 y ejecutar el comando:

\# . init_env

A continuación crear una carpeta con el nombre de la aplicación en el directorio /apps, y acceder a él:

\# cd /home/fox/devorad-R_01/apps/aplicacion/

En este directorio es donde hay que copiar el código fuente de la aplicación y crear el archivo Makefile con el siguiente texto:

$A X I S \_U S A B L E \_L I B S=U C L I B C G L I B C$

include \$(AXIS_TOP_DIR)/tools/build/Rules.axis

PROGS = detector_bluetooth

all: \$(PROGS)

\$(PROGS): \$(PROGS).o

$$
\$(C C) \$(L D F L A G S) \$ \wedge \$(L D L I B S)-o \$ @
$$

clean:

rm -f \$(PROGS) *.o core

Por último, es necesario instalar el compilador cruzado haciendo make crisaxis-linux-gnuuclibc, y compilar definitivamente la aplicación con make. En el mismo directorio se generarán los archivos:

detector_bluetooth

detector_bluetooth.o

El primero de ellos es el ejecutable, que no lleva la extensión .out al compilarlo de esta manera. En este momento ya está el ejecutable listo para cargarlo en la Foxboard.

\subsubsection{Preparación de los empotrados}

La preparación de los empotrados requiere cierta dedicación debido a la complejidad del sistema por su exclusividad en los procedimientos de instalación y configuración, modo de compilación de aplicaciones, reducido tamaño de su 
memoria flash, capacidad de procesamiento, etc. Pero el procedimiento a seguir para configurar todas las estaciones es idéntico, excepto la aplicación, que, como se explica en el apartado anterior, es distinta en todas las estaciones debido al número que identifica a cada una de ellas.

La Foxboard viene por defecto con una imagen de Linux instalada, pero es necesario crear una en la que se activen los dispositivos USB conectados: el Bluetooth y el interfaz Wifi. Con esta imagen cargada en el empotrado, se procede a la configuración de ambos interfaces para el correcto funcionamiento de la aplicación. Y por último se introduce el ejecutable de la aplicación.

Tanto la imagen como las configuraciones de las interfaces y el ejecutable deben ser cargadas en la memoria flash del empotrado para que no se pierdan al cortar la alimentación. Para el desarrollo de la aplicación, instalación de controladores de los dispositivos conectados y para la recompilación del kernel de Linux del empotrado se utiliza la herramienta Phrozen SDK de ACME SYSTEMS [20], la cuál puede funcionar bien en sistemas operativos Windows, usando una máquina virtual de Linux como VMware [VMware'2015], o bien en sistemas Linux.

\subsubsection{Activación de los dispositivos BT y WIFI USB}

En primer lugar se instala el SDK (Software Development Kit) del sistema embebido sobre el sistema Linux del PC donde se trabaja, y se ejecuta el menú de configuración desde el directorio /devorad-R2_01 con el comando:

\section{\# make menuconfig}

Desde este menú se pueden seleccionar los dispositivos que se van a instalar.

Para habilitar el dispositivo Bluetooth hay que seleccionar la opción "driver settings" del menú de configuración, después a "Bluetooth BlueZ libraries and tools", donde se seleccionan las dos opciones que aparecen: "OpenObex" y "ussp-push".

Del mismo modo se habilita el dispositivo Wifi, seleccionando la opción "driver settings" del menú de configuración, pero a continuación se accederá a la opción "Enable WLAN support" seguido de "wireless tool”, donde se activa la opción "C1" correspondiente a la interfaz Wifi USB.

Para generar la imagen es necesario guardar la configuración realizada y salir del menú de configuración. Tras la ejecución de los comandos: 


\section{\#./configure}

\# make

se obtiene la imagen en el directorio /devorad-R2_01, la cuál aparece con el nombre "fimage".

Para cargar la imagen en el empotrado es necesario conectar el sistema en red con el PC Linux o Windows. Por defecto, el interfaz de red Ethernet de la Foxboard viene configurado con la dirección IP 192.168.0.90. Por tanto, la interfaz del PC debe de tener dirección IP de la misma red, por ejemplo 192.168.0.2. Tras la conexión de ambos interfaces mediante un cable de red, se debe comprobar que la red funciona correctamente haciendo ping desde el PC a la dirección IP del empotrado. A partir de aquí se puede realizar la transferencia de la imagen.

Desde Windows, se puede utilizar el programa flashFOX.exe [Crispin'2013]. Para habilitar el envío de paquetes entre el PC y el empotrado vía Ethernet se deben instalar las librerías de WinPcap para Windows. Colocando la imagen en la misma carpeta que el ejecutable flashFOX.exe y accediendo al mismo desde la consola de comandos se iniciará la transferencia con los siguientes comandos:

\section{C:/Image_folder/flashFox -l}

Esto muestra los dispositivos de red activos del PC, de los cuales se debe identificar el que se está utilizando, de modo que si es el segundo se coloca un 2 en el siguiente comando:

\section{C:/Image_folder/flashFOX - $d 2-F-i$ fimage}

Antes de llevar a cabo esta sentencia es necesario realizar el puente J8 "Ethernet flashing" en el empotrado para que se ejecute un pequeño código ROM instalado en su procesador AXIS que permite interactuar con el PC que transfiere la imagen. Tras reiniciar el sistema, arrancará cargando la nueva imagen.

Desde Linux o desde una máquina virtual de Linux en Windows, pero preparados con la herramienta Phrozen SDK y el SDK del sistema Foxboard, también se puede realizar la transferencia de la imagen ejecutando desde consola los comandos:

\# . init_env 
\# boot_linux $-F-i$ fimage

En este momento debe apagarse el sistema Foxboard, hacerle el puente J8 y volverlo a encender. De este modo comenzará a cargarse la imagen y se reiniciará automáticamente cuando concluya la transferencia.

Pero la forma más sencilla de actualizar la imagen, tanto desde Windows como desde Linux, es mediante un explorador WEB, accediendo a la URL http://192.168.0.90. Desde aquí se puede seleccionar la imagen en la opción "Total upgrade" y pulsar "Upgrade" para que comience el proceso de transferencia, mediante el cuál se perderán todos los datos del sistema. Tras finalizar la carga, la unidad se reiniciará automáticamente con la nueva imagen configurada.

\subsubsection{Configuración del dispositivo BT y WIFI USB}

Una vez que el sistema tiene habilitados e instalados los dispositivos Bluetooth y Wifi se procede a la configuración de los mismos. Para ello es necesario acceder al embebido. Ello se realizará manteniendo la configuración de red establecida para la transferencia de la imagen.

La Foxboard tiene un servidor telnet siempre activo y escuchando por el puerto 23, por lo que desde cualquier PC con la consola de Linux o con la consola de comandos de Windows se puede realizar un acceso por telnet simplemente ejecutando:

telnet 192.168.0.90

Pedirá identificación y contraseña, que son las siguientes:

axis loggin: root

Nota: La tecla ENTER no es aceptada para confirmar loggin, hay que utilizar CTRL-J.

Password: pass

A partir de aquí los comandos se ejecutan directamente en la Foxboard.

Para configurar el dispositivo BT basta con cargar las librerías de Bluetooth Bluez con el comando:

\# cdBluez-start 
A modo de prueba, se puede intentar detectar otro dispositivo BT desde la estación para asegurar su correcto funcionamiento. Con el comando hcitool scan se puede realizar un escáner de dispositivos BT.

Para configurar la interfaz Wifi de forma permanente para que arranque con una configuración predeterminada se debe activar e identificar en primer lugar la interfaz inalámbrica:

\# /etc/init.d/wíreless start

\# ifconfig

El adaptador Wifi instalado viene por defecto con dirección IP 192.168.10.90, codificación WEP deshabilitada, canal 1, modo ad-hoc y essid: FOX.

Para que el adaptador Wifi se inicie con la configuración de la red inalámbrica deseada es necesario modificar el archivo /etc/conf.d/net.wireless con el siguiente contenido:

\# the name of your usb device

$D E V="$ rausbo"

\# the ip that you want to use

$I P=" 192.168 .1 .90 "$

SUBNET="255.255.255.0"

\# set your essid

ESSID="ssid de la red wifi"

\# use the wíreless as default route?

WIFI_DEFAULT="0"

WIFI_GATEWAY="192.168.1.254"

\# the channel that we want to use

CANNEL="1"

\# ad-hoc or manager

MODE="Managed" 
\# wep encryption

$K E Y="$ clave wep de la red wifi"

Guardando la configuración deseada, la Foxboard arrancará siempre preparada para acceder a la red inalámbrica.

Este fichero es igual para todas las estaciones, con la diferencia de la IP, que no pueden repetirse entre ninguna de las interfaces Wifi de la misma red.

Por último, es necesario modificar la dirección MAC del interfaz Ethernet. Esto es debido a que tras la carga de una imagen, se establece como dirección MAC la 00:40:8C:CD:00:00, lo que provoca el parpadeo del LED rojo en la placa. Esto sucede para que el programador cambie la dirección MAC tras introducir una nueva imagen, ya que puede generar conflicto si se desea conectar en red con otra estación, como es el caso. De modo que se deben cambiar las direcciones MAC de los interfaces Ethernet de todas las estaciones, y que ninguna de ellas coincida. La primera mitad de la dirección pertenece al fabricante, por lo que sólo se podrán modificar los seis últimos dígitos.

La modificación de la MAC se realiza accediendo por telnet y ejecutando el comando:

\section{bootblocktool -a SERNO 00408CXxxxxx}

Donde $x x x x x x$ son los seis dígitos modificables.

Tras reiniciar el sistema el LED rojo estará apagado y se habrá establecido la nueva dirección MAC. Además se puede observar cómo el LED naranja parpadea con el tráfico de datos por el interfaz Ethernet.

Para evitar confusiones, lo ideal es que todas las direcciones MAC de las estaciones comiencen por 00:40:8C:CD:00:XX, y que los dos últimos dígitos $\mathrm{XX}$ coincidan con los de la parte de identificación de host de la dirección IP de la interfaz Wifi y, a su vez, con el número de estación establecido en la aplicación de detección de dispositivos. Así, por ejemplo, la estación número 5 tendrá como dirección IP para la interfaz Wifi la 192.168.1.5, como dirección MAC la 00:40:8C:CD:00:05 para la interfaz Ethernet, y los mensajes que envíe la aplicación por el socket al programa de monitorización tendrán el formato: 05\#MAC_phone. Las direcciones IP de los 
interfaces Ethernet de todas las estaciones no se modificará (192.168.0.90) debido a que sólo se usa a la hora de la preparación de la estación.

El ordenador de monitorización deberá estar conectado a la misma red que todas las estaciones, y tendrá una dirección IP por encima de la 99, por ejemplo, la 192.168.1.100.

\subsubsection{Configuración de la aplicación en el empotrado}

El lugar donde se tiene que almacenar el archivo ejecutable de la aplicación generado tras la compilación (detector_bluetooth.out) es en el directorio /mnt/flash/ para que quede guardado de forma permanente en la memoria flash. Ubicado en este directorio, no se perderá al cortar la alimentación del sistema.

La copia de este fichero se puede realizar con cualquiera de los siguientes protocolos: SFTP, SCP o FTP. Desde Windows se puede usar el programa WinSCP para conectarse con cualquiera de estos protocolos y poder ver en modo de árbol de directorios las carpetas del disco duro del PC donde estamos trabajando y las de la Foxboard. De modo que podemos coger el ejecutable detector_bluetooth.out y arrastrarlo al directorio /mnt/flash/del empotrado.

Una vez guardado en la memoria flash se puede probar su ejecución accediendo de nuevo mediante telnet y ejecutando la sentencia:

\# ./detector_bluetooth.c

Comenzarán a aparecer las cadenas de texto generadas en cada detección cada cierto tiempo (el establecido en inquiry), siempre y cuando el programa de monitorización esté corriendo y se encuentre conectado a la red inalámbrica. En este programa también se podrán visualizar las detecciones.

Es necesario que las detecciones comiencen a producirse por sí solas en el momento en que la estación sea encendida, ya que tras un corte en el suministro eléctrico provocaría el estancamiento del sistema. Todas las estaciones volverían a arrancar pero no se empezaría a ejecutar la aplicación de detección de cada sistema. Para solucionar este problema basta con crear un archivo en el directorio $/ m n t / f l a s h / e t c / r c 2 . c$ con el nombre S99Aplic y que contenga la ejecución de la aplicación:

$$
\text { \#! /bin/sh }
$$




\section{./mnt/flash/detector_bluetooth.out}

Así, al iniciar el sistema, siempre se ejecutará la aplicación.

Si el programa de monitorización no se está ejecutando, la estación, al detectar algún dispositivo BT, intentará sin éxito crear el socket y tras poco tiempo pasará de nuevo a detectar hasta que se active el programa y se pueda establecer el socket.

\subsubsection{Software de monitorización}

El programa que representa las detecciones de los dispositivos es una interfaz gráfica Java. Permite llevar a cabo el seguimiento de una persona portadora de un terminal móvil con Bluetooth. Utiliza una base de datos MySQL para guardar las detecciones recibidas de cada estación en un instante determinado. Además vincula el número de estación que envía la detección con un emplazamiento concreto, y la dirección MAC de la interfaz BT del terminal detectado la vincula con el nombre completo del individuo.

La clase principal es Servidor.java. Contiene un objeto de la clase Ventana.java que es la interfaz gráfica del programa. También puede contener varios objetos de la clase ThreadServidor.java que se encargan de la gestión concurrente de los sockets.

\subsubsection{Base de datos}

Son tres tablas las que componen el esquema de la base de datos: Detecciones, Empleados y Estaciones.

La tabla Detecciones tiene su atributo $M A C$ vinculado con el atributo $M A C$ de la tabla Empleados, y su atributo estacion está vinculado con el atributo estacion de la tabla Estaciones. Por lo tanto, se trata de claves foráneas para esta tabla. 


\begin{tabular}{|l|}
\hline \multicolumn{1}{|c|}{ Detecciones } \\
\hline id (INTEGER) \\
fechayhora (TIMESTAMP) \\
MAC (VARCHAR(17)) \\
estacion (INTEGER) \\
\hline
\end{tabular}

\begin{tabular}{|c|}
\hline Empleados \\
\hline nombre (VARCHARCHAR(17)) \\
nom_dev (VARCHAR(45)) \\
\hline
\end{tabular}

\begin{tabular}{|l|}
\hline \multicolumn{1}{|c|}{ Estaciones } \\
\hline ubicacion (VARCHAR (INTEGER) \\
tipo (VARCHAR(45)) \\
\hline
\end{tabular}

Figura 16. Vista de los tipos de atributos de las tablas.

Desde la interfaz gráfica se pueden añadir empleados y estaciones a sus respectivas tablas desde los formularios correspondientes. Cuando se produce una detección, el servidor socket recibe la cadena de texto con el número de estación y dirección MAC del dispositivo detectado, datos que se añaden a la tabla Detecciones junto con la fecha y hora de la detección y el identificador, id, que numera la detección por orden de ocurrencia.

Es necesario establecer y manejar conexiones a un servidor de bases de datos MySQL desde la aplicación Java con la API JDBC (Java Database Connectivity). Se trata de un conjunto de clases e interfaces que permiten a cualquier programa Java acceder a sistemas de bases de datos de forma homogénea. Con esta API no es necesario escribir un programa para acceder a Sybase, otro programa para acceder a Oracle, y otro programa para acceder a MySQL; si no que se puede crear un sólo programa que sea capaz de enviar sentencias SQL a la base de datos apropiada.

La aplicación de Java debe tener acceso a un controlador o driver JDBC adecuado. Este controlador es el que implementa la funcionalidad de todas las clases de acceso a datos y proporciona la comunicación entre el API JDBC y la base de datos real. De una manera muy simple, al usar JDBC se pueden hacer tres cosas: establecer una conexión a una fuente de datos (como por ejemplo a una base de datos), mandar consultas y sentencias a la fuente de datos o procesar los resultados.

En el proyecto Java hay que añadir una unidad de persistencia con las librerías hibernate y asociarla con el conector JDBC. Así, aparecerán las clases 
Detecciones.java, Empleados.java y Estaciones.java, cuyos objetos de esas clases se corresponderán con nuevas filas en las tablas de la base de datos.

Las consultas a la base de datos se realizan con sentencias tipo Query. Por ejemplo, para seleccionar la estación de la tabla Estaciones cuyo atributo estacion es igual a est:

Query query1 = em.createQuery("SELECT e FROM Estaciones e WHERE e.estacion = :est");

Donde est es un parámetro que se puede modificar asignándole una variable o unos caracteres, como por ejemplo asignarle un entero que se corresponde con los dos primeros caracteres de la cadena de texto "mensaje":

query1.setParameter("est", Integer.parseInt(mensaje.substring(0, 2)));

De este modo se puede obtener el objeto de la clase Estaciones que cumple la sentencia SQL impuesta en el query. Continuando con el ejemplo, la siguiente línea añade como atributo estacion al objeto deteccion de la tabla Detecciones la estación u objeto de la tabla Estaciones que cumple la sentencia query:

deteccion.setEstacion(((Estaciones)query1.getResultList(0.get(0)));

Esta última sentencia es de escritura en la base de datos, pues se está introduciendo un atributo en la tabla Detecciones, en la fila correspondiente al objeto deteccion, que puede corresponderse a un objeto ya existente o se puede haber creado nuevo:

deteccion = new Detecciones();

Tras añadir cualquier atributo o crear cualquier objeto que vaya a ser añadido a la base de datos, debe de realizarse la transacción. Para el ejemplo anterior:

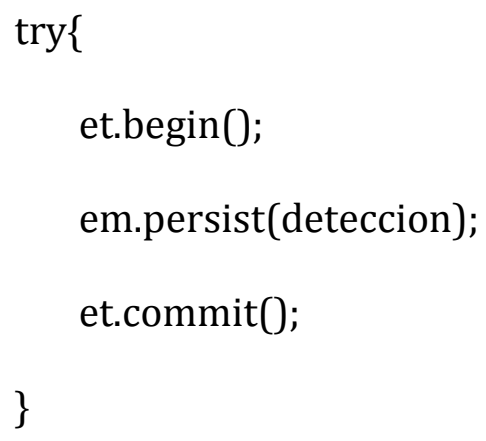




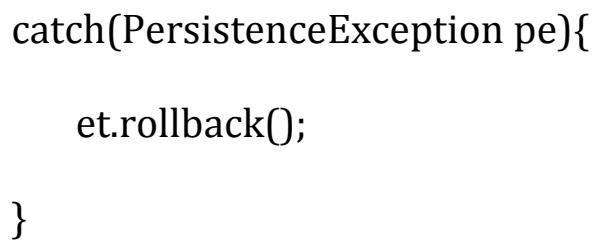

\subsubsection{Interfaz gráfica}

Al arrancar el programa, el método main(0 crea un nuevo y único objeto servidor de la clase Servidor, en cuyo constructor se crea también un único objeto ventana de la clase Ventana, que extiende JFrame.

Este objeto ventana tiene una barra de herramientas tipo JMenuBar con tres JMenu's cuyos nombres son "Archivo", "Editar" y "Ver". Además cada uno de éstos tiene varios JMenuItem's (ver figura 17).

La ventana también tiene dos JPanel's, uno al lado del otro y justo debajo de la barra de herramientas. El de la derecha contiene los cinco botones tipo JButton: "Ultimas detecciones", "Estaciones", "Empleados", "Búsqueda” y "Mapa”. El de la izquierda muestra las diferentes vistas dependiendo del botón pulsado, se le asigna el nombre de contenido.

En los métodos boton_ActionPerformed() se crean nuevas clases tipo JPanel que se añaden al contenido. Estas clases son las siguientes: VerDetecciones.java, VerEstaciones.java, VerEmpleados.java, Búsqueda.java y Mapa.java. Las tres primeras son paneles que contienen las tablas de la misma forma que se encuentran almacenadas en la base de datos.

Otros paneles que se añaden a contenido son los que se crean en los métodos ActionPerformed() de los JMenuItem. Estos JPanel's son los formularios que se implementan en las clases: AñadirEmpleados.java, EditarEmpleados.java, EliminarEmpleados.java, AñadirEstaciones.java, EditarEstaciones y EliminarEstaciones.java.

La ventana, en la parte inferior, también tiene un JTextArea llamado verbose que representa a modo de consola todos los errores que puedan suceder en la ejecución del programa así como los mensajes recibidos por los sockets y las confirmaciones de escrituras en la base de datos. 


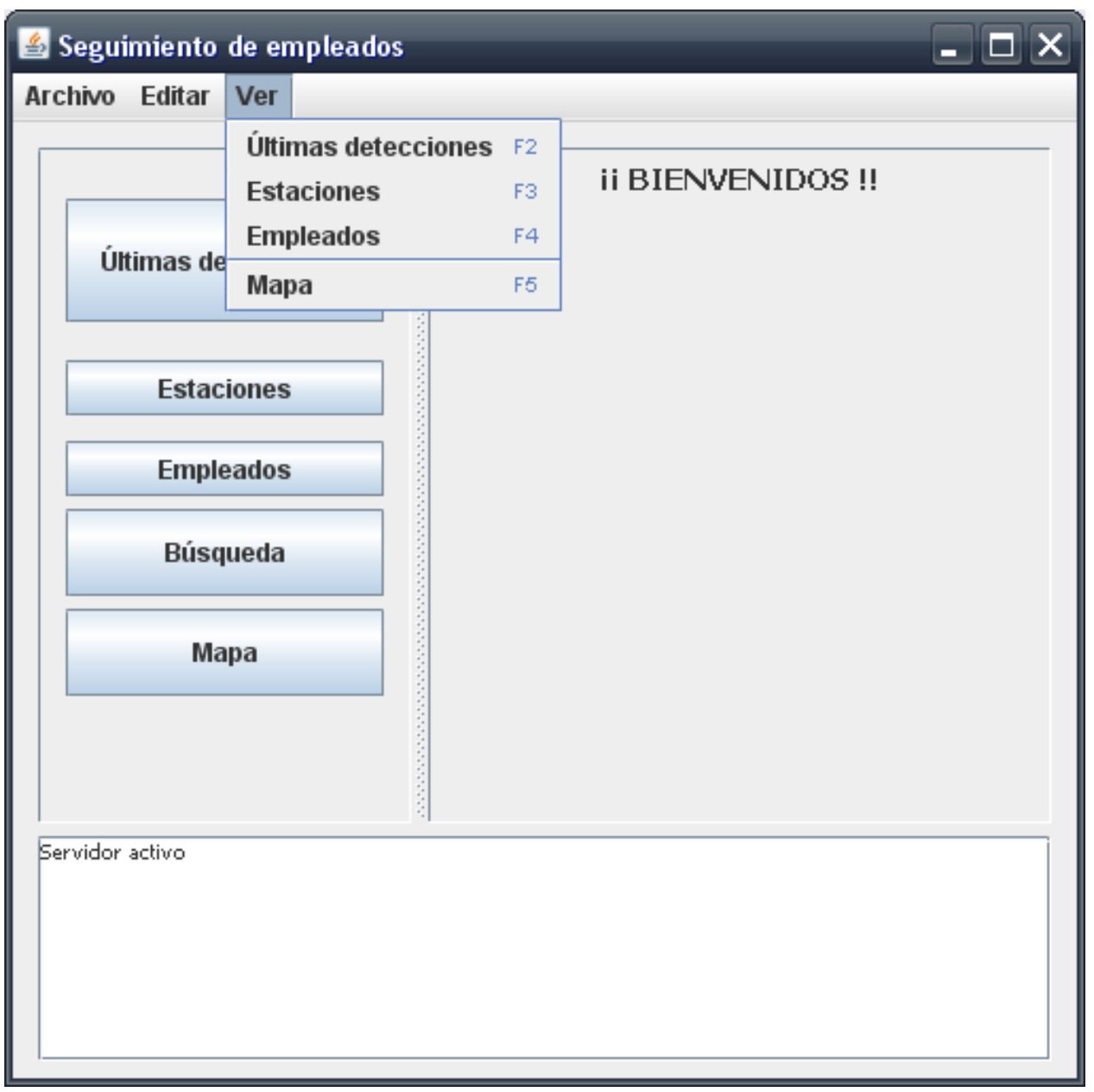

Figura 17. Captura del programa de monitorización al inicio.

\subsubsection{Gestión concurrente de los sockets}

En el método runServer(O de la clase Servidor.java se crea un socket servidor cuyo puerto debe coincidir con el establecido en el cliente socket de la aplicación de las estaciones. En el momento en el que cualquier estación comience a abrir un socket y el servidor lo acepte, se crea un nuevo objeto de la clase ThreadServidor.java que se encargará, en su método run(), de gestionar esta conexión y de recibir la cadena de texto que informa de la detección. De forma concurrente podrán establecerse conexiones en el caso en que varias estaciones envíen detecciones al mismo tiempo.

\subsubsection{Procesado de la información}

En la clase TheradServidor.java, una vez que se recibe el mensaje de la estación, se descompone identificando con los dos primeros caracteres a la estación que lo envía, y con los 17 últimos a la dirección MAC del dispositivo BT detectado. En este momento, se comprueba si existe algún empleado en la tabla Empleados de 
la BD cuya dirección MAC coincida con la recibida en el mensaje, utilizando una consulta query. Si existe, se crea una nueva detección en la tabla Detecciones y se rellenan sus atributos con los respectivos métodos set. Para los atributos estacion y empleados es necesario pasarles los objetos completos, ya que se crearon como claves foráneas. Por tanto, hay que hacer consultas a la base de datos para obtener estos objetos, estacion y empleado, que se correspondan con el número de estación y dirección MAC de la detección recibida.

\subsection{Prueba y Evaluación del sistema}

El mejor modo de probar la funcionalidad del sistema es montándolo en un escenario determinado tal y como corresponde a un trabajo con carácter innovador como el presentado en este documento de tesis. El lugar elegido es el Antiguo Cuartel de Antigones, edificio en el que reside la ETSIT de la UPCT.

Las pruebas más representativas son aquellas en las que se pueda comprobar si en las distintas zonas, pasillos o accesos a controlar, se produce de forma correcta y efectiva la detección con el paso del individuo. Por tanto, en primer lugar, se eligen estos puntos para la colocación de las estaciones.

Tras la colocación de las estaciones y el PC donde se realiza la monitorización los resultados, resulta imprescindible la colocación de un punto de acceso Wifi que permita conectar todas las estaciones en la misma red. En las pruebas realizadas, el punto de acceso se conecta mediante un cable Ethernet al PC de monitorización. En otros entornos, podría ser necesaria la utilización de más puntos de acceso por la separación de las estaciones.

Una vez establecida la red, se encienden todas las estaciones y se ejecuta el programa. Lo primero que hay que hacer en el programa es añadir las estaciones, rellenando el formulario "Añadir Estación" (que se accede desde la barra de herramientas en el menú "Editar") en el que se especifica el número de estación, el tipo de estación (si es de tipo zona, pasillo acceso o estancia) y la descripción de la ubicación. A continuación añadir los empleados que se deseen controlar, rellenando el formulario de "Añadir Empleado" donde se especifica su nombre completo, la dirección MAC del Bluetooth de su terminal móvil y, opcionalmente, el identificador de su Bluetooth. 
A partir de aquí cualquiera de los empleados que se acerquen a las estaciones, irán siendo detectados y su recorrido entre los distintos puntos controlados quedará almacenado en la base de datos.

Los resultados obtenidos dependen en gran medida del tiempo de inquiry establecido. Si se establece un tiempo por encima de 10 segundos, se obtienen unos resultados muy favorables en cuanto al número de dispositivos descubiertos simultáneamente, pero las detecciones se estarán produciendo cada intervalos de tiempo muy elevados, lo que puede impedir la detección de empleados cuando crucen las zonas de alcance en menos de ese tiempo. Pero el establecimiento de un tiempo menor de 3.88 segundos $(3 \times 1.28)$ disminuye de forma considerable el porcentaje de descubrimientos de dispositivos al alcance.

Experimentalmente, con 25 dispositivos en la zona de detección de una estación, el número de dispositivos detectados varía en función del tiempo de inquiry o tiempo de detección. Este tiempo, múltiplo de 1.28, se ha variado hasta 6.4 segundos obteniendo un porcentaje de detecciones como el que se muestra en la gráfica de la figura 18.

Porcentaje de detecciones

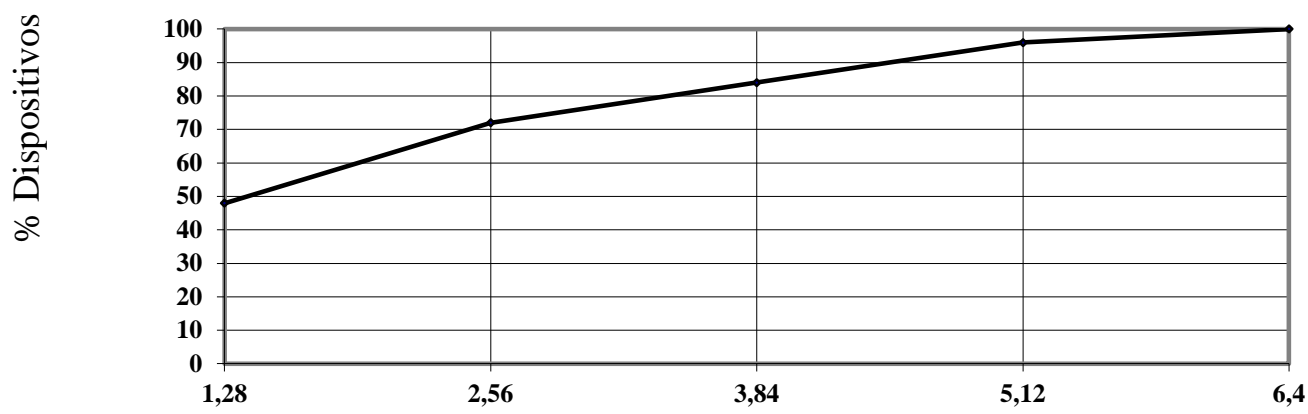

Tiempo de detección (s)

Figura 18. Resultado experimental del porcentaje de dispositivos detectados en función del tiempo de detección.

Con 6,4 segundos se obtiene una probabilidad muy alta de que se detecten todos los dispositivos. Incluso con 3.84 segundos, se obtiene un resultado muy 
satisfactorio en zonas de paso donde el número de dispositivos a detectar simultáneamente pueda ser muy inferior. Estos porcentajes están muy próximos a los obtenidos de forma analítica y teórica en el artículo [Peterson'2006], en su tabla Devices Discovered by Inquiry Time. Las diferencias existentes se deben a la consideración de canal perfecto en su análisis teórico.

Por otro lado, todas las detecciones que se producen son enviadas al programa de monitorización de forma rápida y segura, ya que disponen de una red de mucha capacidad para el escaso tráfico que se produce.

Tras la realización de una prueba, se observa que en la tabla de detecciones aparece muchas veces la misma detección con horas de 3 segundos de diferencia. Esto se debe a que las estaciones envían detecciones cada 3 segundos aproximadamente, por lo tanto, si un empleado se encuentra al alcance de la estación durante más tiempo, se producirán muchas detecciones.

Se comprueba que se obtienen unos resultados muy satisfactorios, pero existe demasiada información como para poder obtener a simple vista un seguimiento de un determinado empleado. En el siguiente apartado se comenta cómo solventar estos problemas.

Para solventar el problema de detecciones repetidas, lo que se hace es que en la clase ThreadServidor, cuando se recibe una nueva detección, se comprueba si se ha producido una detección desde la misma estación y de la misma dirección MAC como máximo unos 10 segundos antes. Para ello hay que observar su atributo fechayhora. Cuando sólo se ha producido una detección igual en esos 10 segundos, se sigue almacenando la detección. Pero si ocurre una nueva detección a menos de 10 segundos de la segunda repetida, lo que se hace es sobrescribir esta última. De modo que la primera detección quedará guardada y la segunda se irá sobrescribiendo hasta que no se produzca una nueva detección 10 segundos antes que la anterior. Quedando reflejado en la base de datos primera detección y la última. 
Úlimas detecciones:

\begin{tabular}{|c|c|c|c|c|}
\hline$N^{\circ}$ & Empleado & Estación & Fechay Hora & MAC \\
\hline 1 & Sergio Lujan & 2 & $2008-11-0508: 28: 33.0$ & $00: 1$ C:9A:F8:7D:A5 \\
2 & Sergio Lujan & 4 & $2008-11-05$ 08:28:46.0 & $00: 1$ C:9A:F8:7D:A5 \\
3 & Sergio Lujan & 6 & $2008-11-0508: 29: 02.0$ & $00: 1$ C:9A:F8:7D:A5 \\
4 & Sergio Lujan & 6 & $2008-11-0510: 46: 12.0$ & $00: 1$ C:9A:F8:7D:A5 \\
5 & Sergio Lujan & 4 & $2008-11-0510: 46: 27.0$ & $00: 1$ C:9A:F8:7D:A5 \\
6 & Sergio Lujan & 3 & $2008-11-0510: 47: 09.0$ & $00: 1 C: 9 A: F 8: 7 D: A 5$ \\
7 & Sergio Lujan & 3 & $2008-11-0511: 15: 02.0$ & $00: 1 C: 9 A: F 8: 7 D: A 5$ \\
8 & Sergio Lujan & 4 & $2008-11-0511: 15: 18.0$ & $00: 1 C: 9 A: F 8: 7 D: A 5$
\end{tabular}

Estaciones:
\begin{tabular}{|c|c|c|}
\hline Estación & Tipo & Ubicación \\
\hline 2 & Acceso & Entrada a la Universidad \\
3 & Estancia & Cafetería \\
4 & Pasillo & Pasillo de departamentos \\
6 & Estancia & Departamento
\end{tabular}

Figura 19. Capturas del programa de monitorización: detecciones un empleado concreto y tabla de estaciones.

En la figura 19 se observa cómo el empleado "Sergio Lujan" ha sido detectado por la estación 2 (detección 1), "Entrada a la universidad”, a su hora de entrada al trabajo, sobre las 8:30h. Segundos después pasa por el "Pasillo de departamentos" (detección 2), y otros pocos segundos después llega a su "Departamento" (detección 3) donde permanece hasta las 10:46h (detección 4, que representa la última detección en esa zona). Pasa por el pasillo de nuevo (detección 5) y accede a la "Cafetería" de la Universidad (detección 6). A las 11:15h sale de la cafetería (detección 7) de vuelta pasando por el pasillo de los departamentos (detección 8).

El programa, además, ofrece la posibilidad de colocar un mapa de distribución en planta al que añadir las estaciones, permitiendo la visualización de forma gráfica de los recorridos de los empleados y la hora exacta de cada paso por los puntos controlados.

A continuación se pueden ver algunas capturas de la interfaz gráfica, de sus formularios y tablas: 


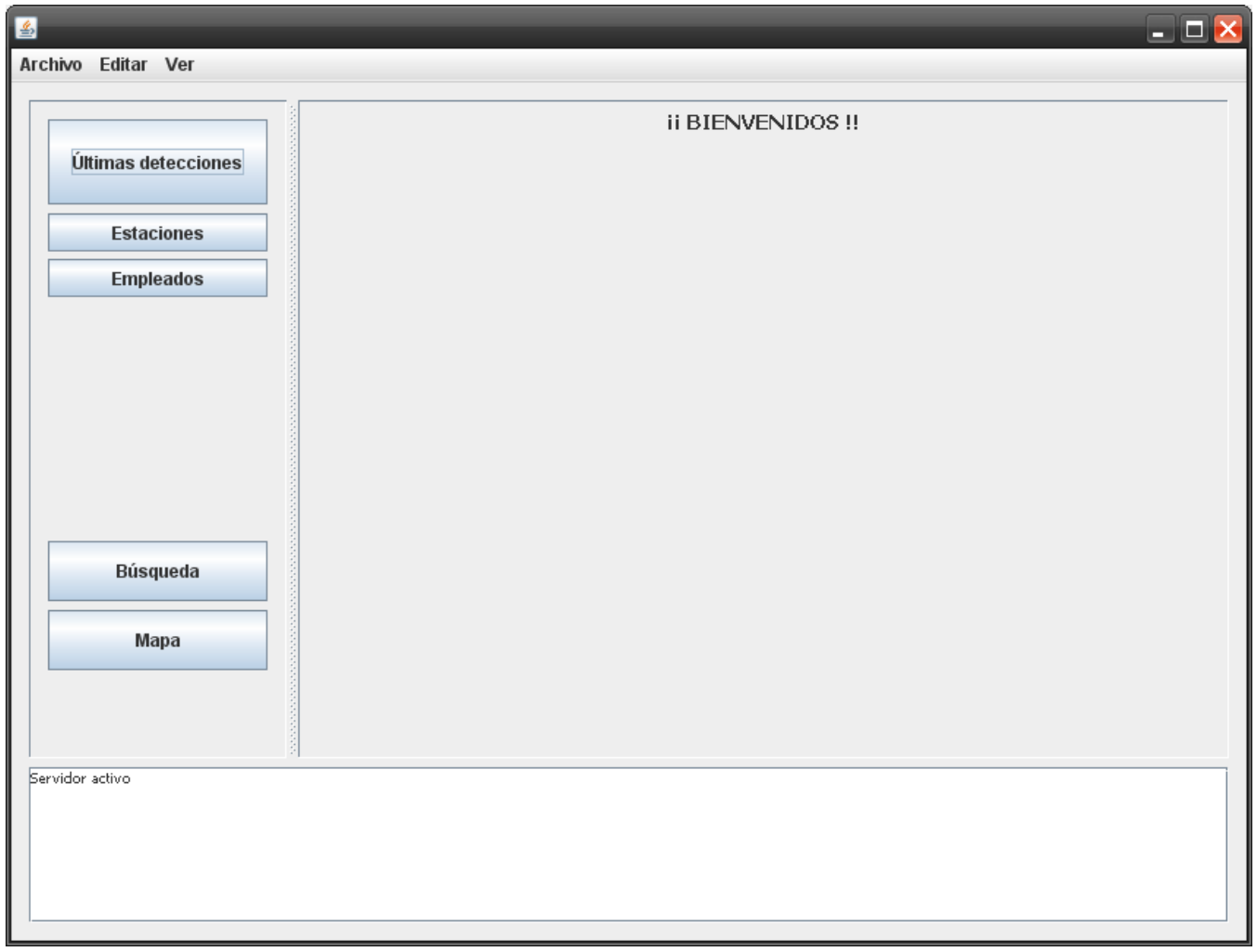

Figura 20. Capturas del programa de monitorización: panel de bienvenida.

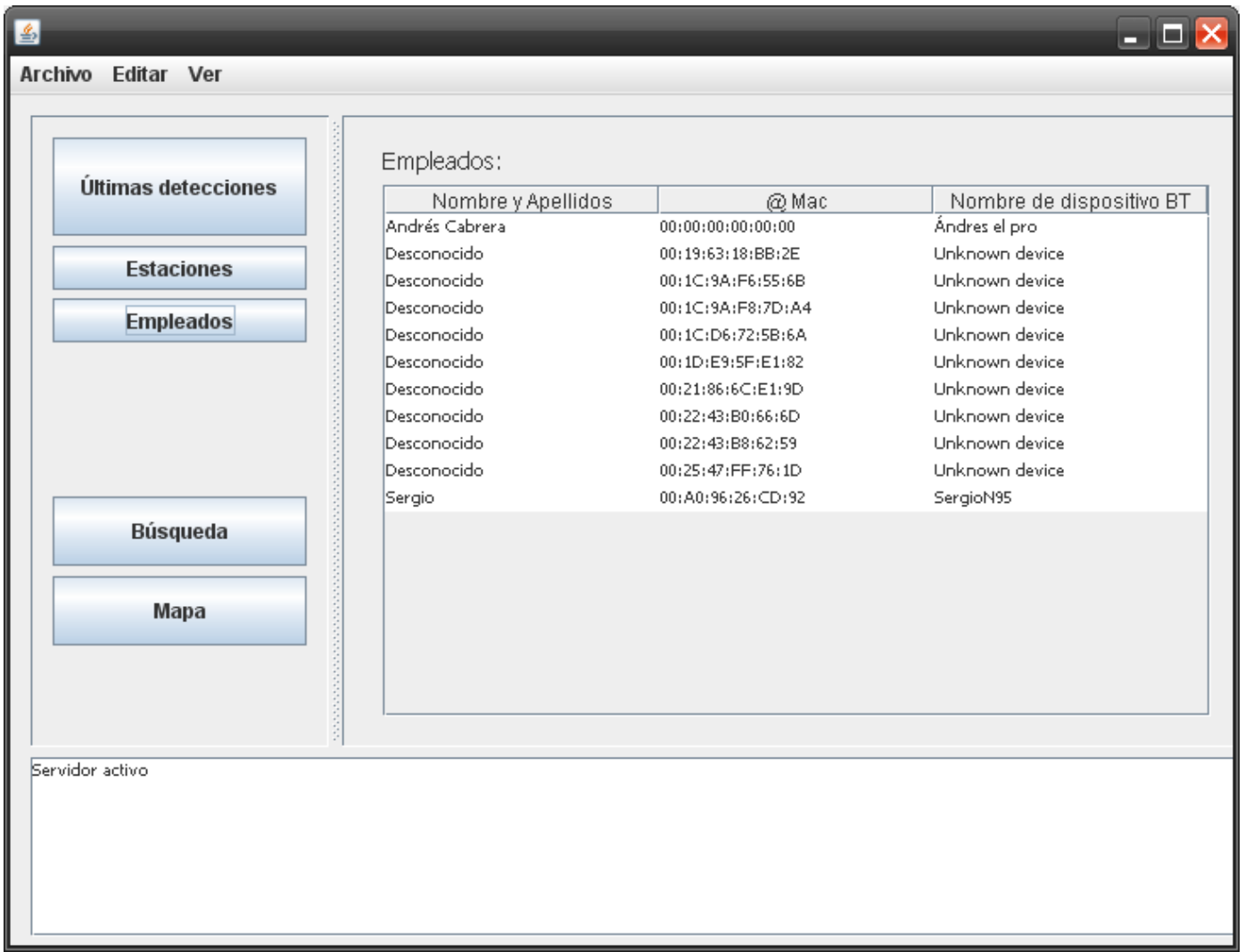

Figura 21. Capturas del programa de monitorización: empleados registrados en la base de datos. 


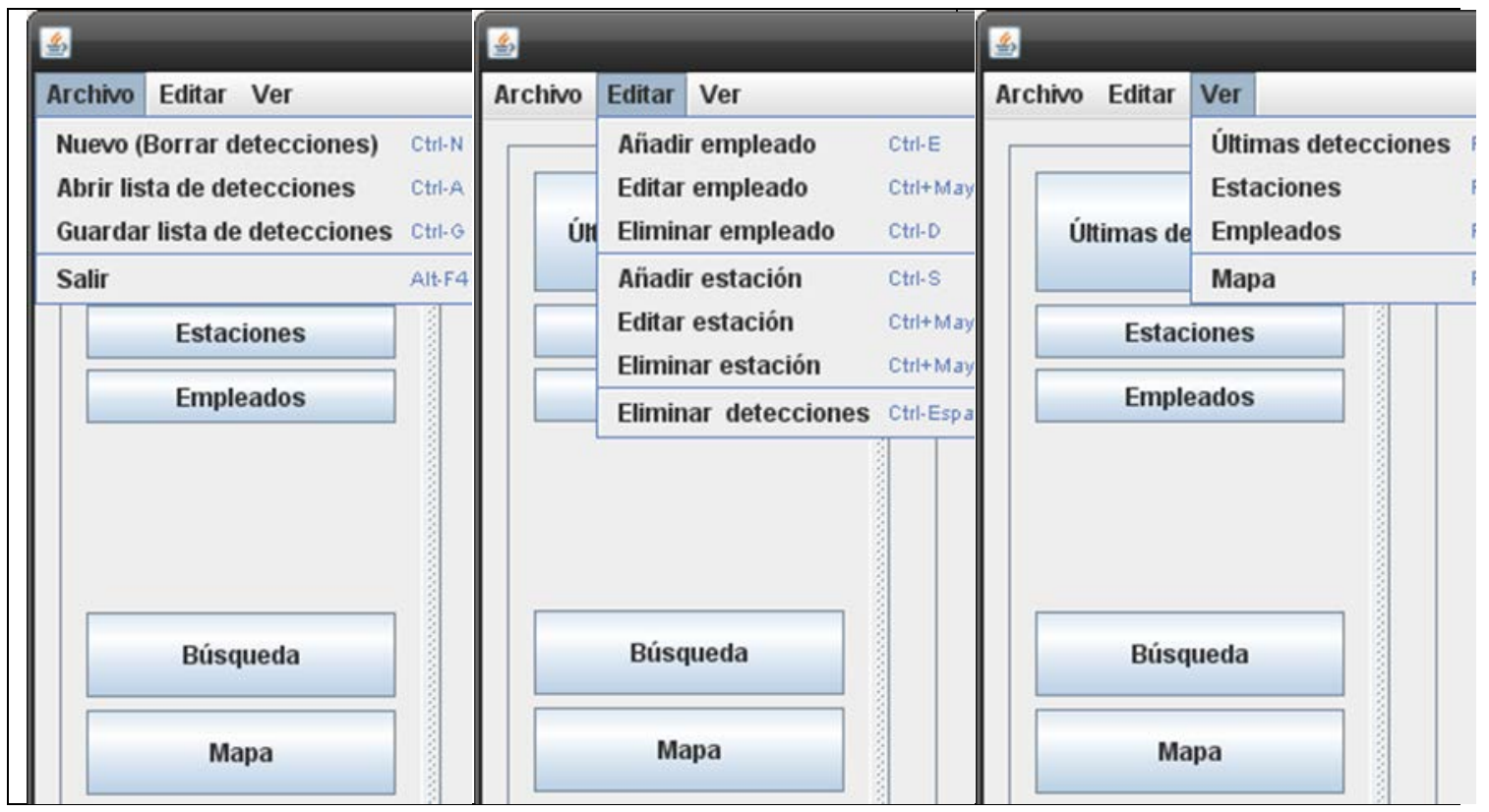

Figura 22. Capturas del programa de monitorización: herramientas.

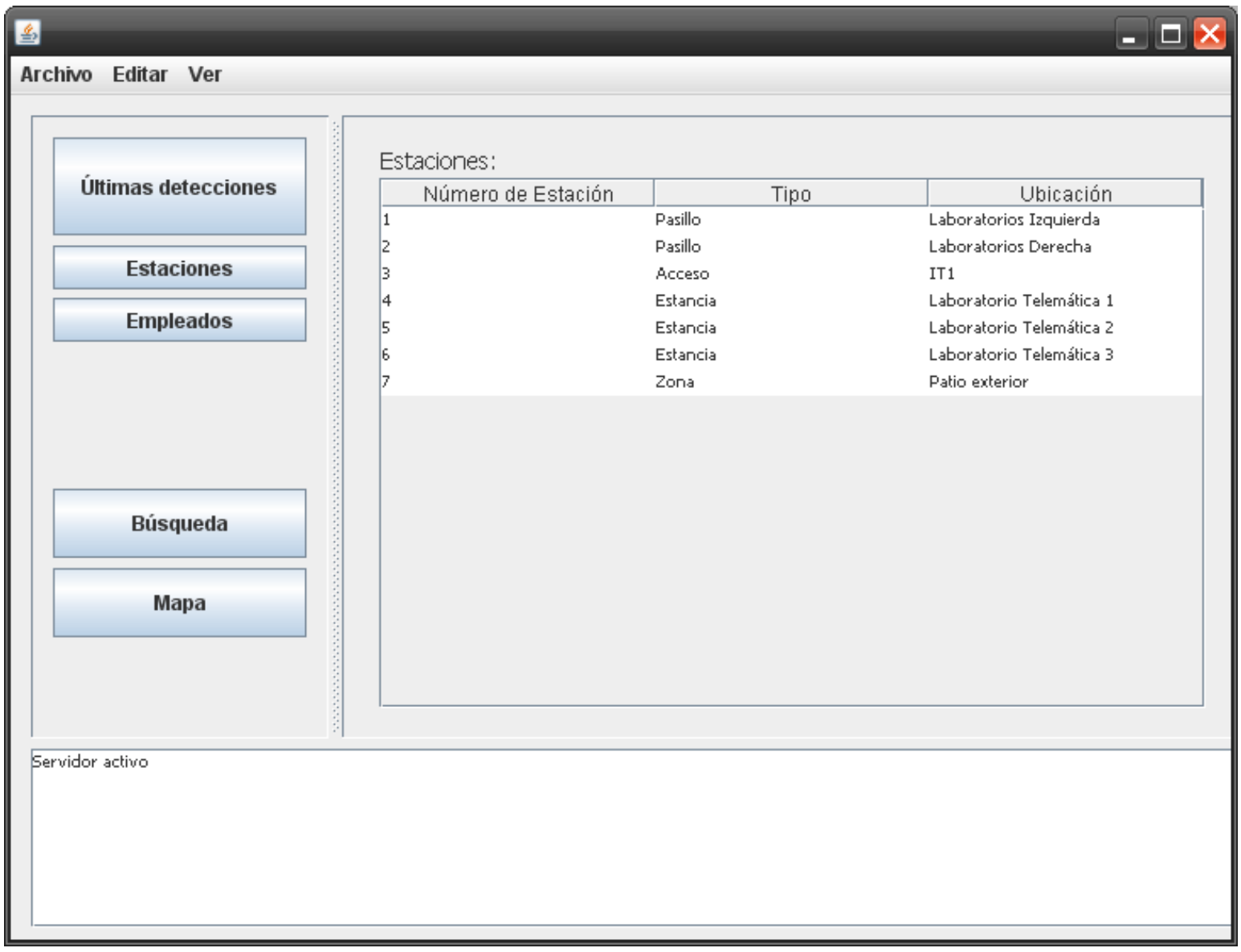

Figura 23. Capturas del programa de monitorización: estaciones instaladas. 
Seleccione la detección que desea eliminar:

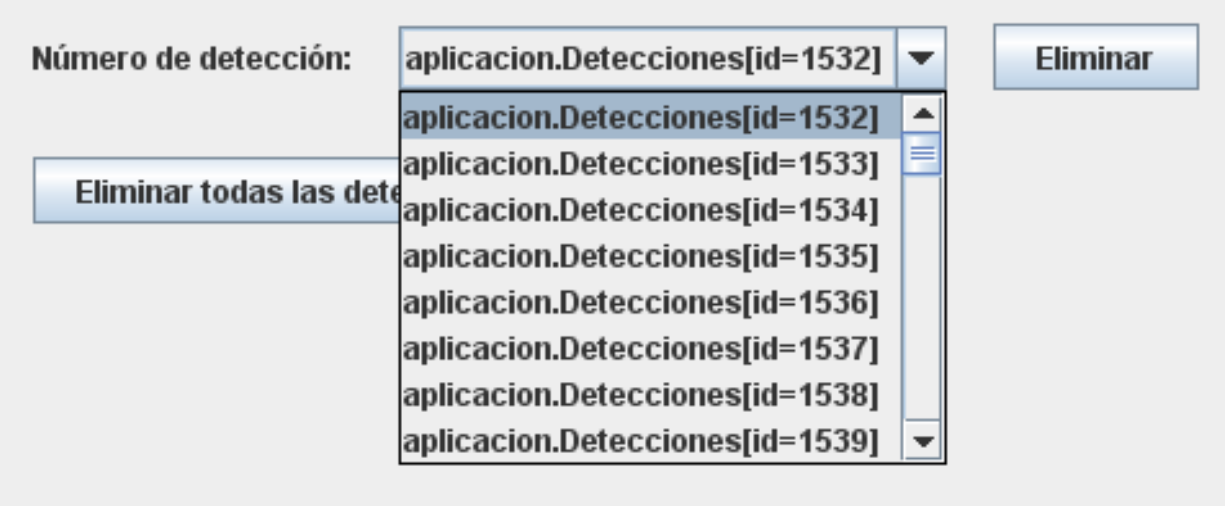

Figura 24. Capturas del programa de monitorización: formulario para eliminar detecciones (de una en una o todas a la vez).

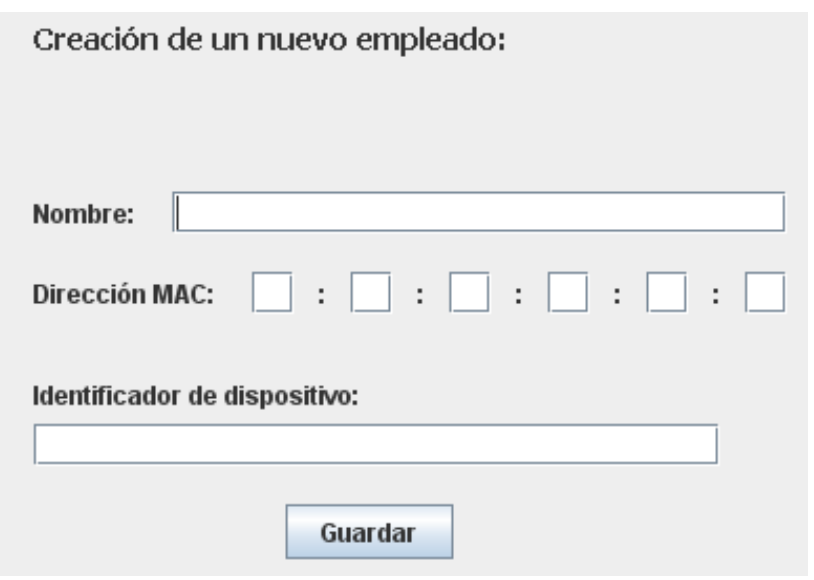

Figura 25. Capturas del programa de monitorización: formulario para registrar empleados.

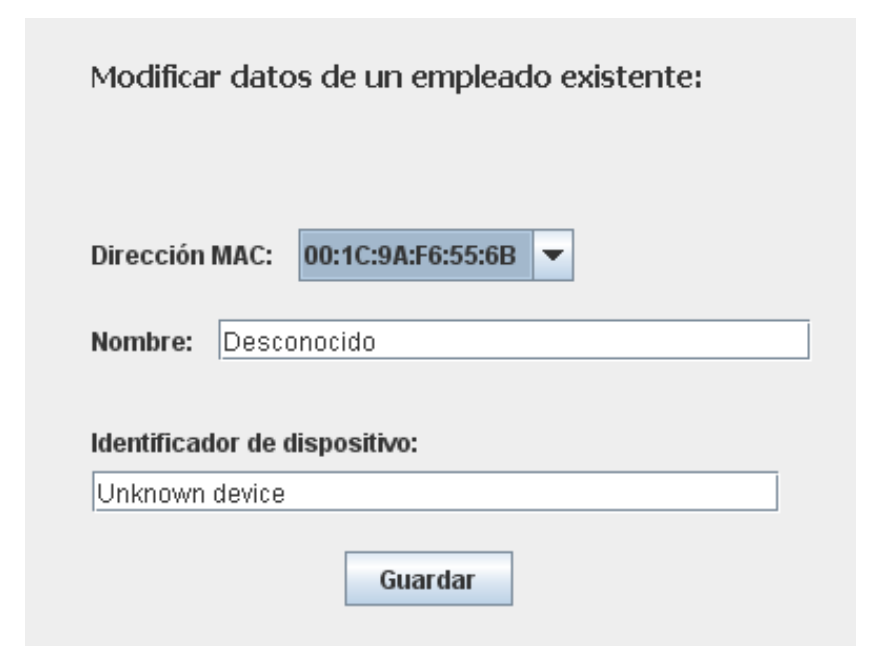

Figura 26. Capturas del programa de monitorización: formulario para editar datos de los empleados registrados. 
Seleccione la MAC del empleado que desea eliminar:

Dirección MAC:

00:1C:9A:F6:55:6B

Eliminar

Figura 27. Capturas del programa de monitorización: formulario para eliminar empleados a partir de la dirección MAC.

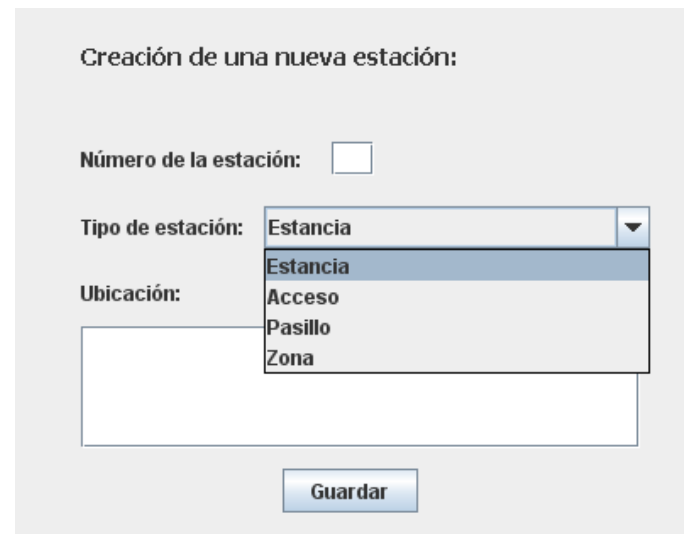

Figura 28. Capturas del programa de monitorización: formulario para añadir una nueva estación.

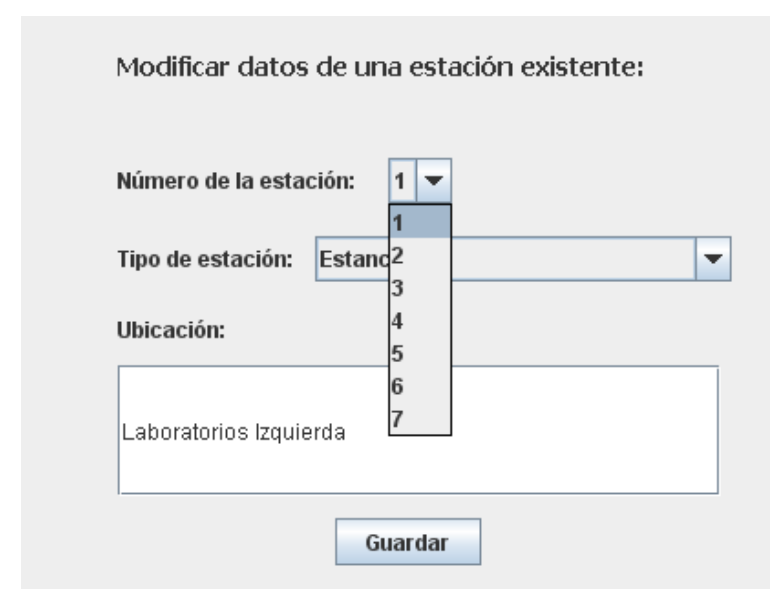

Figura 29. Capturas del programa de monitorización: formulario para modificar los datos de una estación

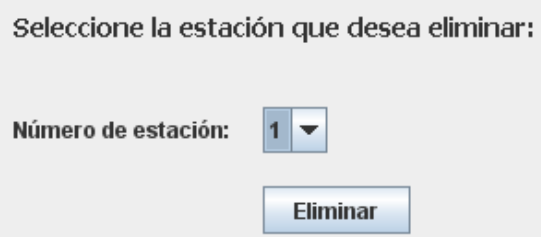

Figura 30. Capturas del programa de monitorización: formulario para eliminar una estación. 


\subsection{Uso del sistema para control de asistencia en entornos docentes}

La seguridad se ha vuelto un aspecto fundamental en cualquier área y en cualquier lugar. Diferentes sectores y mercados verticales han decidido implementar soluciones de seguridad que brinden la protección necesaria para las instalaciones y edificios, así como para las personas que residen, trabajan o visitan dichos lugares.

En los centros educativos los sistemas de control de acceso se han convertido en un elemento esencial. Su uso busca regular y controlar el ingreso, así como monitorizar aspectos laborales y de asistencia.

La implementación de la mayoría de sistemas de seguridad parte de la necesidad de protegerse de la delincuencia común o de los diferentes actos delictivos y esto también aplica en el caso de las instituciones educativas.

El objetivo de un control de acceso en centros educativos es conocer quién ingresa a los mismos, realizar una identificación de estudiantes, empleados y visitantes para garantizar que el personal autorizado puede circular por las instalaciones y los alumnos de enseñanzas obligatorias asisten a clase.

En las instituciones educativas la implementación del control de ingreso puede hacerse de dos formas, una se enfoca en el control de acceso a la institución y otra sólo para las áreas restringidas. La primera opción implica que el control se hace desde la entrada principal y se autoriza el ingreso a través de una barrera física ya sea tipo torniquete o arco. En la segunda el estudiante puede entrar a la universidad sin tener una barrera en los accesos principales y el control de acceso se hace en facultades o edificios administrativos. En ambas opciones, la implementación del sistema para regular la entrada le permite a las instituciones hacer la impresión del carnet estudiantil sobre la misma tarjeta de control de acceso.

Las soluciones para regular el acceso se instalan dependiendo de las necesidades de cada institución y de manera personalizada, pues esto depende del tipo de universidad, si es privada o pública. En una institución privada, el control puede hacerse en accesos peatonales y vehiculares, además de oficinas administrativas. Para modelos de instituciones públicas se puede utilizar 
únicamente en puertas a nivel de facultades u oficinas administrativas y accesos vehiculares para empleados de la institución.

Controlar el ingreso de visitantes (incluso de los mismos empleados) dentro de una institución es una de las posibilidades de este tipo de soluciones de seguridad e identificación. Una de las principales ventajas de un sistema de control de acceso es que el usuario final puede tener la marcación con el registro de hora y fecha, lo cual es utilizado para poder hacer desde control de asistencia a clase o hasta la implementación de tiempos y asistencia para respaldo de liquidación de nómina para empleados.

Usualmente las zonas dónde se usa control de acceso son definidas por el responsable de seguridad de la institución educativa; la tecnología no está limitada para ser usada en aulas de clase, salones o auditorios. Sin embargo, es poco usual que las universidades implementen controles de acceso electrónicos a un aula de clase por razones de presupuesto limitado. No obstante algunas instituciones instalan controles en las aulas para que sólo el docente pueda abrirla en el momento de la clase, esto con dos objetivos: eliminar el uso de llaves y generar un control de tiempo y asistencia del educador.

Conforme la tecnología avanza y nuevas alternativas, como la propuesta en este trabajo de tesis, se verán cada vez más universidades y centros educativos en general que emplean controles electrónicos en aulas, dado que el principal beneficio es poder determinar en tiempo real si el estudiante se encuentra o no dentro del mismo lugar.

La identificación de los diferentes grupos de personas que ingresan a las universidades como empleados, estudiantes, visitantes o exalumnos se puede efectuar agrupándolos dependiendo del tipo de usuario y configurándolos desde el mismo sistema de administración de control de acceso, o también se pueden emitir y personalizar los carnets con diseños diferentes que permitan hacer la diferenciación de forma visual.

\subsubsection{Tecnologías implementadas}

Hoy en día el mercado de la seguridad electrónica presenta una amplia gama de tecnologías para implementar los controles de acceso. Las credenciales más usadas varían dependiendo de las necesidades. 
Mifare es una de las credenciales más utilizadas, pues puede integrarse con sistemas de transporte masivo, siendo un beneficio adicional que puede agregarse al carnet. Sin embargo cada día las tarjetas inteligentes sin contacto siguen ganando fuerza, como es el caso de iCLASS SE, pues es una tarjeta que nace para ser implementada en soluciones de control de acceso de forma nativa, con los beneficios de poder contar con todos los servicios anteriormente mencionados.

Algunas instituciones están explorando la posibilidad de trabajar con tarjetas de múltiple tecnología en donde se manejen desde Mifare para integración con transporte masivo e iCLASS SE para la implementación de control de acceso de forma segura y los servicios internos de las instituciones educativas.

El uso de tarjetas bancarias (tarjetas débito) dentro del mismo carnet es algo que también puede verse como un servicio adicional.

\subsubsection{Seguridad e identificación}

Existen diferentes niveles de seguridad para un sistema de control de acceso en los centros educativos. Lo primero es la personalización del carnet estudiantil que en este caso puede ser una tarjeta de control de acceso. Dentro de la personalización se pueden tener varios elementos de seguridad que hacen este documento único y difícilmente falsificable. El usuario final puede agregar desde hologramas metálicos personalizados embebidos en el mismo plástico, como las que tiene las tarjetas de crédito Visa o MasterCard, hasta laminados que puedan incluir elementos seguros micro texto, marcas UV (Ultra Violeta) e imágenes holográficas entre otras.

El otro punto a evaluar es la tecnología de aproximación utilizada en el sistema de control de acceso; la que más se está utilizando es la que se conoce en el mercado como tarjeta inteligente de no contacto, una tarjeta que le permite a la institución implementar múltiples servicios asociados a la tarjeta adicionalmente al control de acceso.

Las tarjetas inteligentes no sólo permiten asociar otros servicios al uso de la credencial, también posibilitan a las instituciones manejar niveles de seguridad diferentes. En el caso de iCLASS SE el sistema permite blindar a la universidad a través de sus llaves Elite, es decir, sólo las tarjetas codificadas con estas llaves son las únicas que pueden ser validadas por los lectores de la institución. 
Otra forma de incrementar la seguridad dentro de un control de acceso es el uso de formatos corporativos como por ejemplo el Corporate 1000 de HID, un programa que proporciona a los profesionales de seguridad la capacidad de estandarizar una única tarjeta. Este formato mejora la administración de la codificación de las tarjetas, ya que HID podrá asegurar la no duplicidad de códigos de las tarjetas. Es importante resaltar este punto a la hora de la implementación de una solución de control de acceso, ya que no pueden existir códigos repetidos, pues cada código es el que finalmente va a identificar al usuario dentro del sistema.

La información de cada tarjeta está centralizada en un base de datos que la cual puede ser transmitida a través de la misma red de datos de la institución, teniendo en cuenta que la gran mayoría de controladores del mercado ya tienen la interfaz Ethernet integrada.

Cabe destacar que los datos almacenados dentro de un sistema de control de acceso son muy valiosos, ya que entregan información de respaldo al usuario para la toma de decisiones en casos como liquidación de nómina, control de asistencia o investigación.

Como en cualquier sistema que almacene datos es necesario hacer una administración de ellos mantenimiento, Back Up y eliminación de algunos que no sea relevantes.

Las instituciones que incorporan controles de acceso electrónicos pueden inclusive llegar a relacionar la base de datos del sistema de control de acceso con su plataforma de servicios escolares para ofrecer y obtener prestaciones adicionales.

\subsubsection{Más allá del control de acceso}

Las tarjetas de control de acceso pueden ser utilizadas en otros espacios dentro de las instituciones educativas. La implementación de servicios en bibliotecas, pago en cafeterías y pago de estacionamientos son algunos de los servicios que pueden asociarse a la tarjeta, también se han habilitado para el uso de los lockers y dormitorios, y cada día se hacen más comunes con el uso de cerraduras electromecánicas.

Otros de los servicios que pueden asociarse a la tarjeta es el pago de fotocopias, máquinas expendedoras, etc. De alguna manera y en especial las 
universidades buscan convertir la tarjeta de control de acceso o la identificación/carnet del estudiante, en una credencial de servicios, que además de abrir puertas, tiene otras aplicaciones ligadas a la identificación como las arriba descritas.

Hoy en día son muchas las instituciones educativas que están implementando soluciones de control de acceso e identificación para incrementar la seguridad y la asistencia a clase a la vez que optimizan procesos administrativos y mejoran la eficiencia empresarial donde la solución propuesta en este capítulo de tesis, puede ser una alternativa económica y eficaz.

\subsubsection{Sistema CADI}

Se trata de una aplicación educativa innovadora donde se ha puesto en práctica el sistema de adquisición y filtrado descrito en las secciones anteriores de este capítulo de tesis, como elemento de control de asistencia a clases allí donde es obligatorio o bien el profesor lo requiere en función del tipo de actividad formativa que se desarrolle.

CADI es una plataforma basada en tecnologías web para la creación, desarrollo e implementación de ambientes educativos heterogéneos. Ofrece una interfaz gráfica de usuario para que los alumnos puedan interactuar con el único requisito de contar con un dispositivo genérico compatible con WiFi. Su innovador diseño permite al profesor seleccionar y combinar los recursos desarrollados para crear actividades muy variadas, mostrándose en cada caso los contenidos adaptados a cada dispositivo concreto y superándose así las fallas de estandarización existentes en la actualidad en el mundo de los dispositivos móviles. La utilidad del sistema fue evaluada a través de la realización de un proyecto piloto a un grupo de alumnos veteranos. La experiencia mostró que el uso del sistema ayudó a los estudiantes a conseguir mejores resultados académicos en su evaluación. También mostró que los alumnos no tuvieron ningún problema en interactuar con el sistema desde el principio, promoviendo en última instancia su aprendizaje activo [Cabrera'2012].

\subsubsection{Integración de componentes}

En cuanto al hardware en sí, CADI se integra en una carcasa de 187 x 187 x $40 \mathrm{~mm}$, con los siguientes componentes: el PC empotrado (con un disco duro SSD de 
estado sólido, la placa base, el procesador y un módulo de memoria RAM), un AP WiFi reducido y un adaptador USB a Ethernet. La figura siguiente ilustra la integración y la distribución espacial de los componentes.

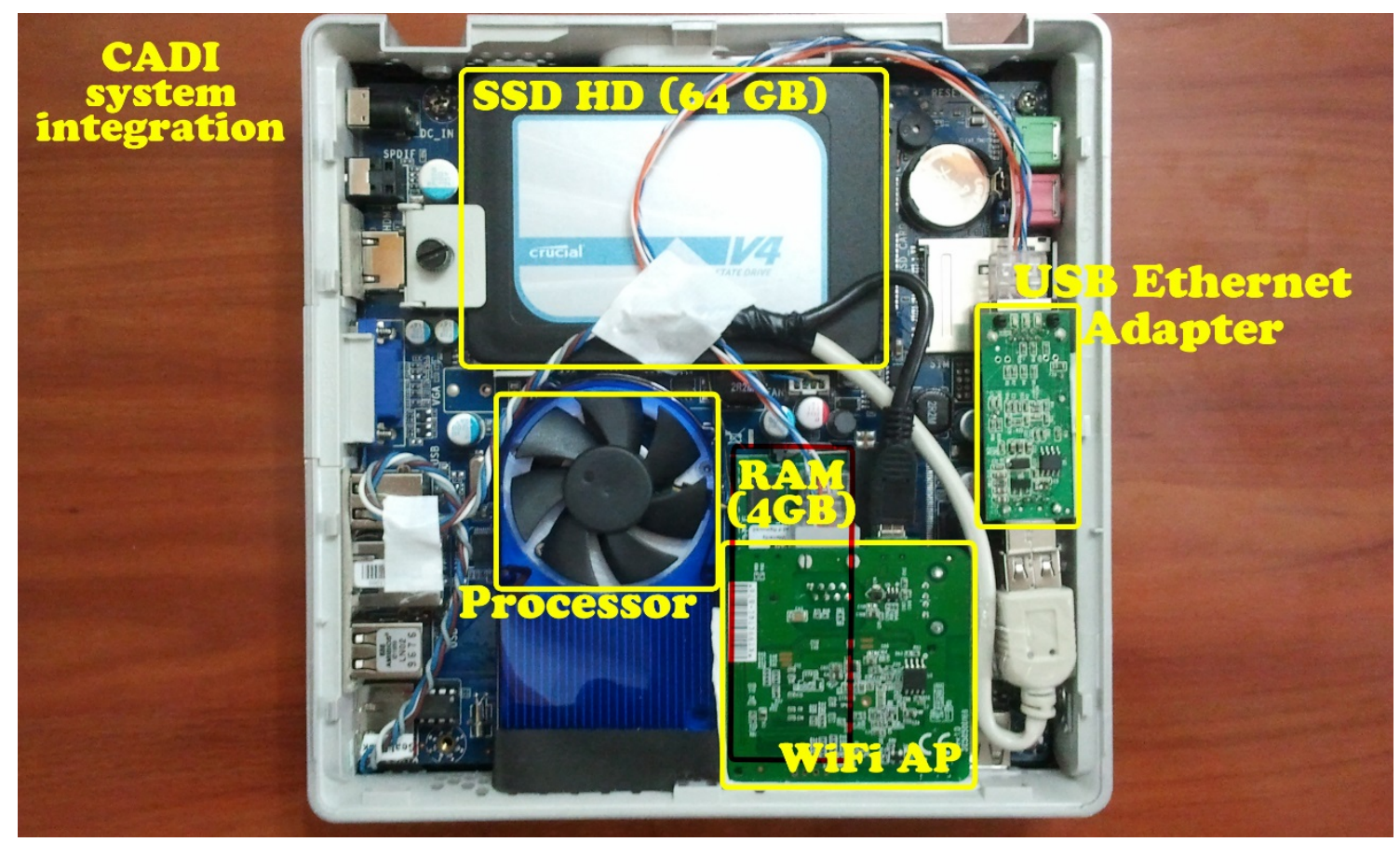

Figura 31. Integración y distribución de los componentes en el prototipo

Esta configuración específica permite que el sistema se ejecuta en el PC al mismo tiempo que despliega una red WiFi dedicada a través del punto de acceso. Por otra parte, la adición del adaptador de USB a Ethernet permite la liberación de la interfaz Ethernet nativa de la placa base la cual sigue estando libre para una operación en línea opcional de la plataforma.

\subsubsection{Arquitectura software}

La arquitectura software del sistema está implementada sobre una instalación estándar del famoso gestor de contenidos web Typo3 WCMS (Web Content Management System, http://www.typo3.org) en el servidor. Dicho gestor está escrito en el lenguaje PHP y usa una base de datos MySQL para almacenar de forma persistente toda la información que maneja. Además, Typo3 provee varios mecanismos para expandir su funcionalidad básica, a través del gestor de extensiones, el denominado Extension Manager. Este módulo del CMS permite la adición de nuevas características y módulos al sistema siguiendo un proceso de importación e instalación de ficheros llamados extensiones (extensions). 
Por otro lado, Typo3 cuenta con un framework para la creación de extensiones llamado kickstarter (que, a su vez, es una extensión) y que ha sido usado para desarrollar cada una de las UBCs (Unidades Básicas de Comunicación) del sistema CADI, decir, las preguntas, encuestas, cuestionarios, foros, el módulo de estadísticas e informes así como el control de acceso.

La arquitectura software del sistema desarrollado cumple con los principios clásicos de diseño software en términos de extensibilidad (ya que se pueden desarrollar e instalar como extensiones otras UBCs en cualquier momento), escalabilidad y reusabilidad (ya que se pueden crear de forma estable y fácil ilimitadas instancias de las UBCs instaladas). 


\section{CAPÍTULO 3: Sistema de estimación de Matrices Origen- Destino}

Para lograr el máximo de eficiencia posible en los recursos que se dedican al transporte público, resulta especialmente útil disponer de herramientas de previsión de la demanda que permitan evaluar de forma anticipada las consecuencias económicas y ambientales de determinadas inversiones y elegir las más adecuadas para el desarrollo de las ciudades y de sus habitantes. Dicho de otro modo, la optimización de los recursos disponibles para el transporte público, ya sean energéticos, humanos o de infraestructuras, requiere un conocimiento preciso de los movimientos de los pasajeros, es decir, de cómo se distribuye el flujo de pasajeros entre las diferentes paradas o estaciones de la línea.

Habitualmente, las autoridades y empresas de transporte llegan a este conocimiento mediante encuestas que se hacen a los usuarios. La metodología utilizada para este fin es muy variada. En este sentido, resulta muy interesante [Ibeas'2007], donde se recogen muchas de las recomendaciones sacadas de [Ortuzar'2011]. Sin embargo, todos los métodos utilizados para hacer encuestas comparten algunas características básicas, de las que posiblemente la más determinante es que la recolección de datos se realizará en un período muy largo de tiempo y para todos los días de la semana, para poder así llegar a conclusiones suficientemente precisas y adaptadas a los cambios estacionales. Justamente esto es lo principal desventaja, dado que implica tanto un coste muy elevado, como un tiempo demasiado largo antes de poder disponer de los datos buscadas. Todos los autores coinciden en este punto. Así, en [Caceres'2007] se remarca la necesidad de gran cantidad de tiempo y el precio elevado de los métodos tradicionales de obtención de datos sobre el flujo de vehículos en carreteras (ya sea por monitorización de matrículas, como por encuestas o recuento manual), y en [Dawei'2008] se especifican algunos de los apartados de este gasto en el caso de autobuses, como son las derivadas de la contratación de personal para repartir las encuestas y procesarlas después, aparte de que se comentan problemas intrínsecos del método, como el hecho de que no todos los pasajeros rellenen la encuesta que les dan o que, por ejemplo, el índice de respuesta dependa del nivel de aglomeración en la salida. 
En las siguientes secciones se describe el desarrollo de una sistema que permite la obtención y filtrado de datos con información origen-destino de usuarios de transporte publico basándose en la extracción de información de un archivo de texto plano (*.txt), para posteriormente ser almacenada en una base de datos de la cual se filtran y presenta únicamente la información necesaria según corresponda, además del desarrollo de funciones de análisis con la información de la BD y construcción de la matriz de probabilidades origen destino.

\subsection{Sistema de adquisición de datos adaptado a la red de transporte}

La implementación de este prototipo se ha centrado en la detección de dispositivos Bluetooth activos y visibles en el autocar.

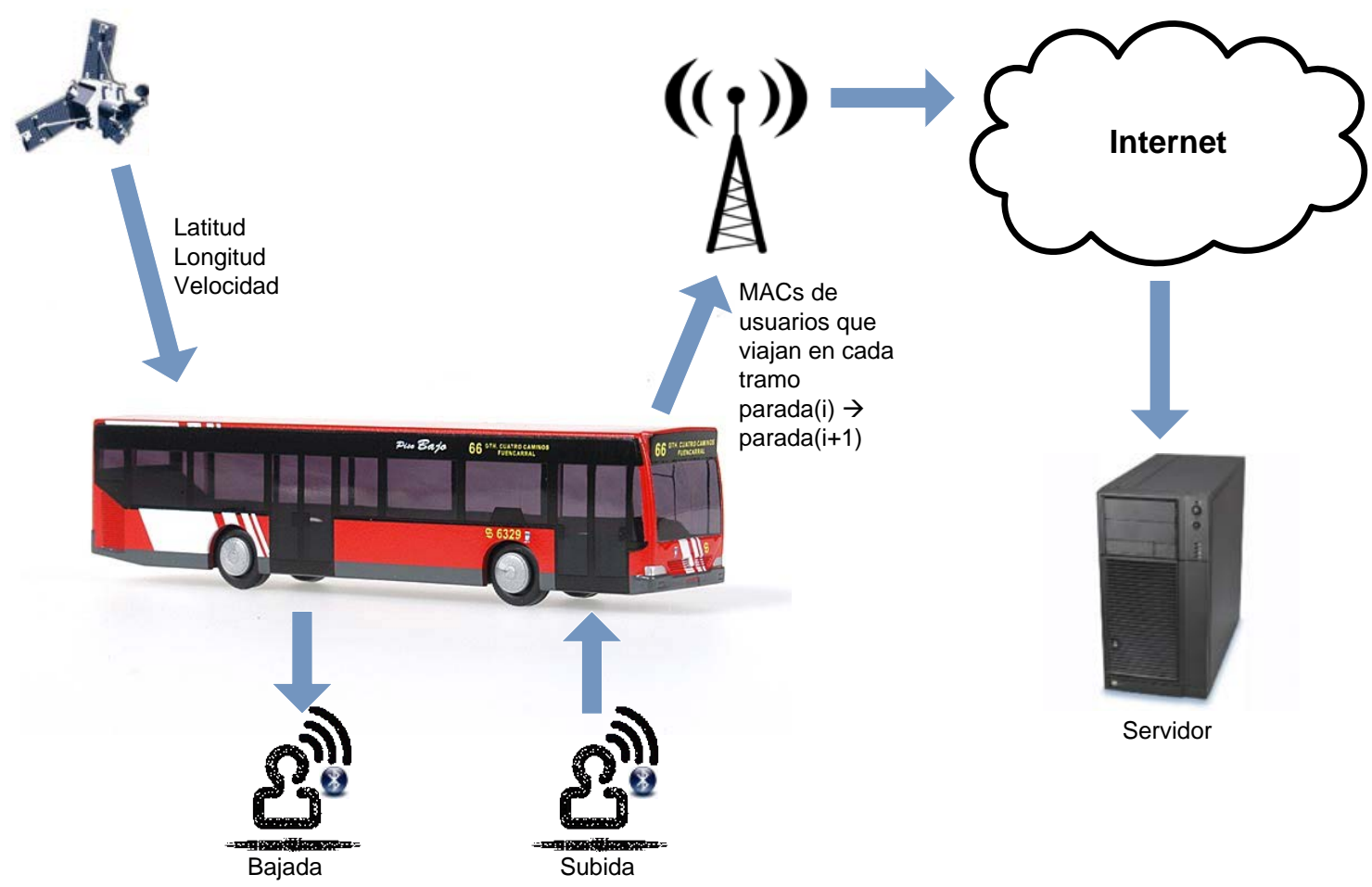

Figura 32. Gráfico conceptual del proceso de detección.

Las detecciones obtenidas son previamente procesadas para descartar información errónea y pasan a componer la matriz origen-destino que, a lo largo del tiempo, establece unas probabilidades de ocupación para cada tramo e irán asociadas a una probabilidad de error. Así se determina en qué medida la detección de los interfaces Bluetooth ayudarán al fin propuesto. 
Con todo, el sistema incorpora el hasheado de las muestras de conteo al iniciarse su procesado, por lo que no se conserva ninguna traza que pudiera, siquiera remotamente, vincularse con los datos personales. Los tags permiten "identificar" al pasaje cuando sube o baja del autobús (cuando "permanece en" o "desaparece del" ámbito del lector). Así, la lectura de cada tag no solo es útil para su contabilización, sino que también procesa la identificación (no personal) del mismo y la relaciona con los otros parámetros significativos del autobús (parada, línea, itinerario...) y del evento (marcas de fecha, hora y tipo: subida o bajada). Por consiguiente, la gestión de datos explota esta terna: cifras de conteo, marcas de fecha y hora, y coordenadas de geoposición.

Esta herramienta aprovecha a modo de tag el dispositivo Bluetooth que muchos pasajeros llevan consigo. De esta manera, la detección de tales dispositivos se utiliza para estimar el número de pasajeros que hay en todo momento a bordo, cuándo han subido o dónde bajan.

En el proceso de captación y transmisión de los datos que permitan esta estimación se distinguen tres partes claramente diferenciadas: la detección de dispositivos propiamente dicha, el procesado de los datos obtenidos resultantes de esta detección y, por último, la transmisión al host de los ficheros con los datos procesados.

Un detector a bordo del autobús realiza lecturas periódicas de los dispositivos Bluetooth dentro del radio de cobertura de su antena. Estas lecturas no tienen por qué coincidir en su totalidad con la muestra deseada: la de los pasajeros embarcados. Concretamente, la antena puede captar dispositivos en vehículos o peatones próximos. Este problema se subsana con el procesado posterior de los datos. Los parámetros configurables en esta etapa son la periodicidad de las lecturas y el posicionamiento y directividad de las antenas. Es interesante el análisis de la conveniencia de intensificar el número de lecturas Bluetooth en las proximidades de las paradas, cuando la variabilidad de los pasajeros es mayor.

Para obtener la máxima información que facilite la determinación de cuáles de los dispositivos Bluetooth detectados fueron de pasajeros embarcados en el autocar, se realiza de procesado de las detecciones. Para ello, se descartan las 
detecciones que son recogidas cuando el autocar se acerca a una parada, ya que multitud de transeúntes o usuario que esperan otros autocares pueden ser detectados.

Es conveniente seleccionar diversas políticas de descarte para testear en el escenario real y poder así determinar la más apropiada.

Una vez obtenidos los datos en la etapa anterior se han de transmitir a un host remoto para su tratamiento posterior, tal como se explica en posteriores epígrafes. Esta transmisión se realizará vía GPRS, después de cada parada o cada cierto tiempo, de manera que el sistema trabaje en tiempo real recibiendo información por slots de tiempo reducidos. En el próximo capítulo, analizaremos una alternativa de comunicación desarrollada que permite conexiones más robustas, económicas y a mayores distancias.

Para el diseño del prototipo encargado de las detecciones, preprocesado y envío de datos se ha utilizado un sistema embebido con las siguientes características:

- Placa base en formato Mini-ITX.

- $\quad$ Procesado ATOM 525 FANLESS, Dual Core de 1,8 GHz.

- $\quad$ Chipset Intel NM10.

- $\quad$ Módulo de memoria DDR2 $800 \mathrm{MHz}$ de 2GB.

- $\quad$ Disco duro de 250GB.

- Conexión Gigabit Lan (10/100/1000Mb).

- $\quad$ Cuatro puertos USB 2.0.

- Ranura para PCI.

- Ranura Mini PCIExpress.

- $\quad$ Puerto ATA 133.

- Cuatro puertos SATA2.

- $\quad$ Conector PS2.

- Salida VGA.

- Salida de Audio 5.1.

- Fuente de alimentación estabilizada DC-DC 12V.

- Función POWER ON BY AC. Activación automatica al volver la tensión de entrada. 


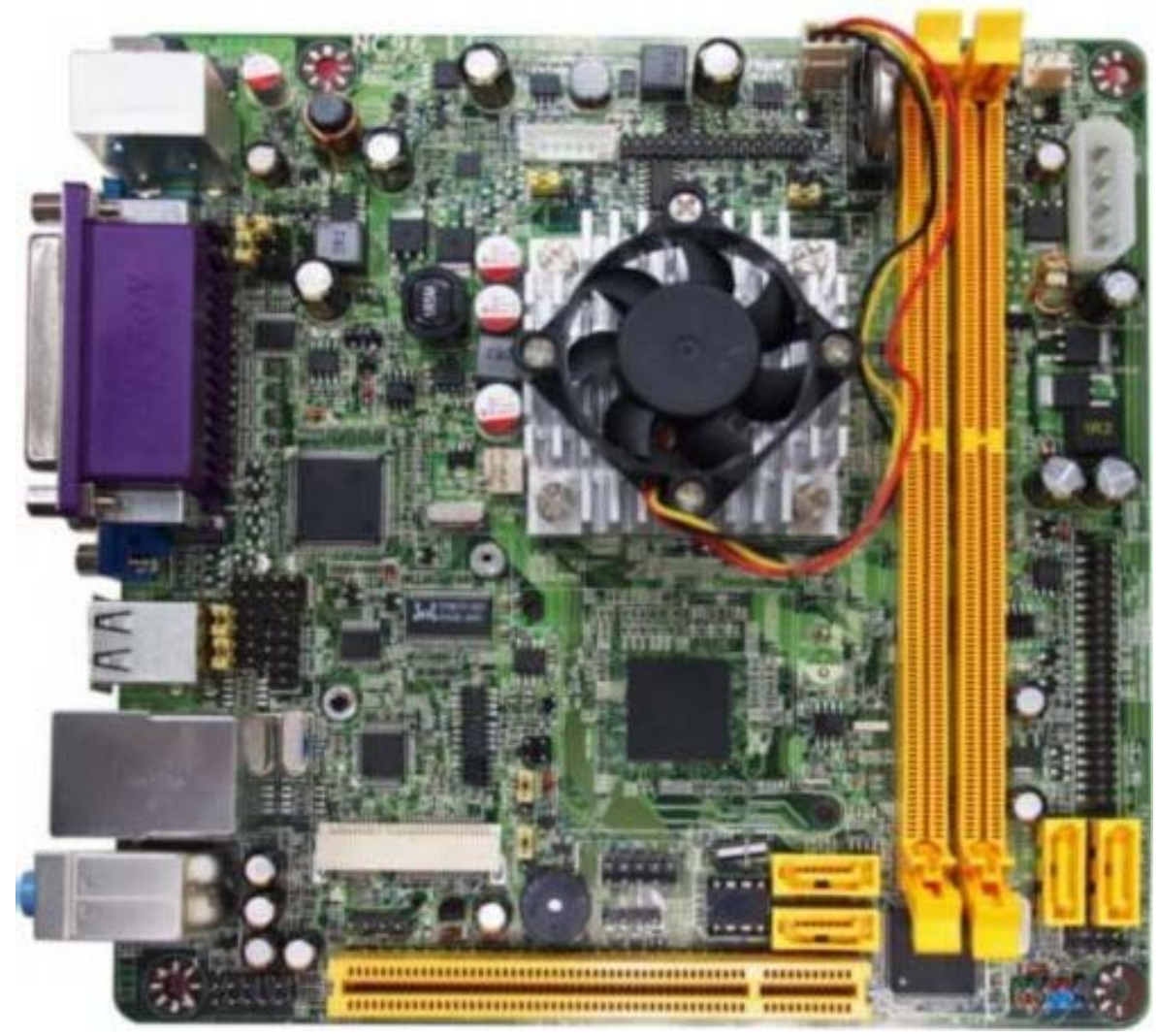

Figura 33. Placa base.

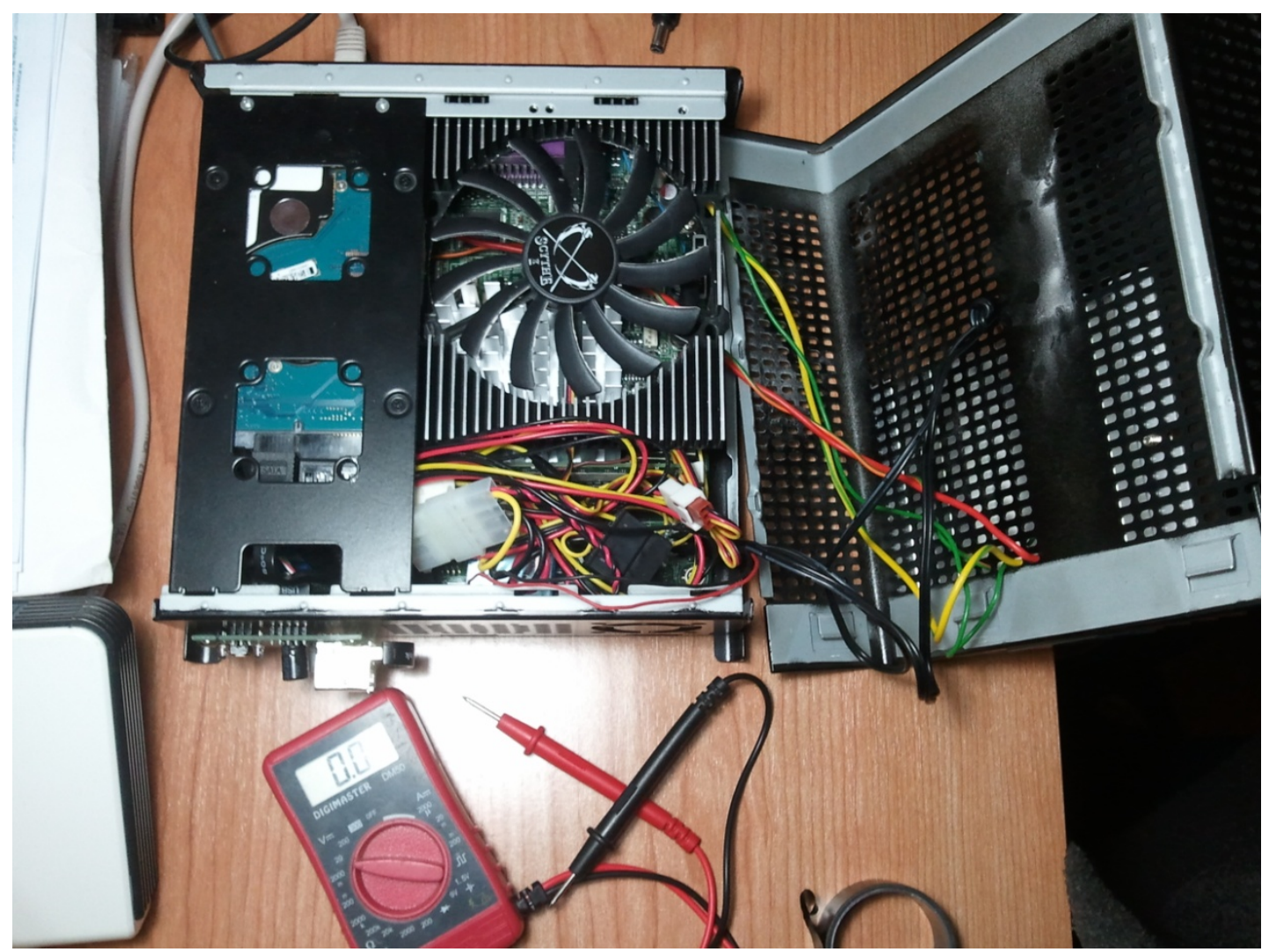

Figura 34. Sistema embebido. 
Cabe destacar la importancia de la fuente de alimentación integrada en la placa base con capacidad para trabajar con tensiones que varíen entre 10 y 36 voltios. Como sabemos, tanto el prototipo como el dispositivo final deben estar preparados para funcionar a las tensiones de trabajo de los autocares ya que, al ir embarcados en ellos, no se dispondrá de suministro de red a 230VAC. Ello conlleva la necesidad de soportar cambios bruscos en la tensión suministrada que son propios del vehículo y que se deben a los altibajos energéticos que se producen con la batería y alternador.

Este hardware es complementado con un router capaz de proporcionar conectividad a Internet vía Ethernet a través de un módem 3G convencional. Sus características son las siguientes:

- 1 Puerto WAN / LAN de 10/100Mbps, Puerto USB 2.0 para Módem 3G, un Mini Puerto USB para el suministro de energía.

- Botón de Configuración Rápida de Seguridad, Botón de Reseteo, Switch de Modo.

- Alimentación 5VDC/1.0A.

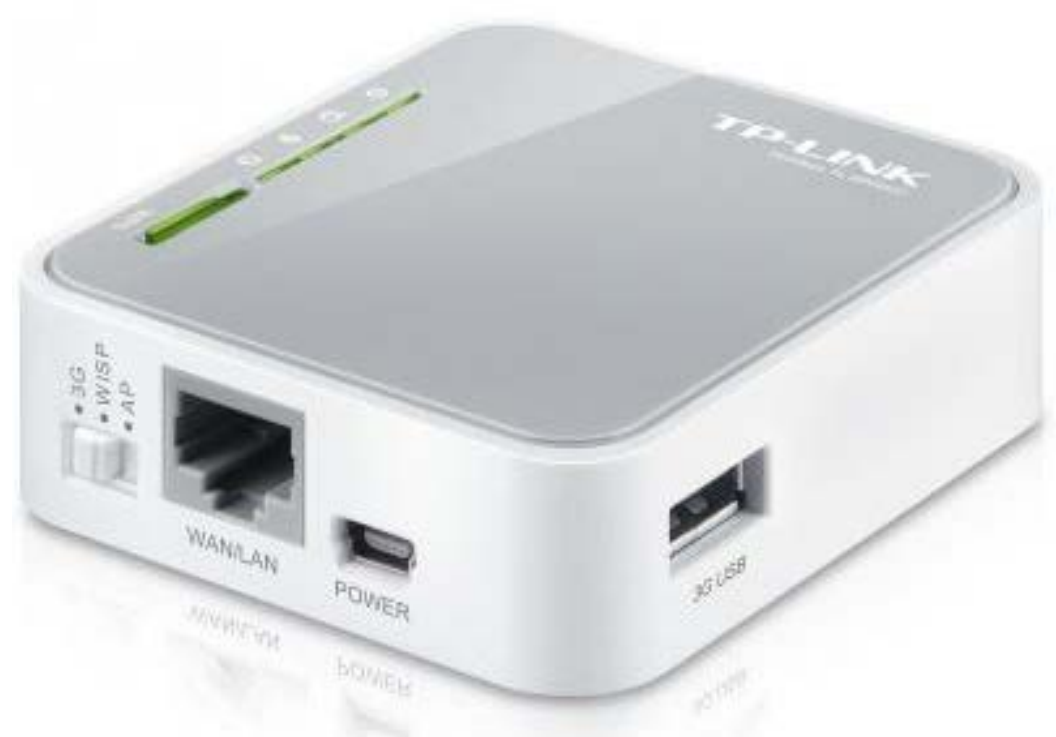

Figura 35. Router con puerto USB para módem 3G.

El módem utilizado para el testeo del prototipo ha sido suministrado por el proveedor de servicios Vodafone España y sus características son las siguientes: 
- Velocidad de descarga HSDPA: 14,4 Mbps.

- Velocidad de subida HSUPA: 5,7 Mbps.

- Compatible con routers a través de USB.

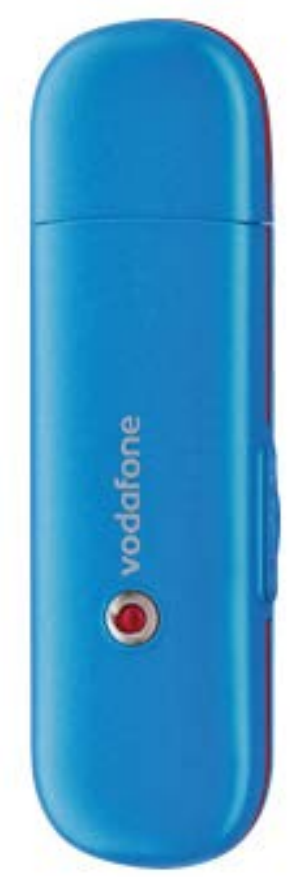

Figura 36. Módem 3G Vodafone.

La posibilidad de utilizar la tensión de $5 \mathrm{~V}$ proporcionada por la fuente de alimentación de la placa base, simplifica notablemente la circuitería a emplear para el sistema de alimentación.

También se ha dotado al sistema de un circuito interruptor controlado por puerto serie con el fin de posibilitar reinicios del hardware de comunicaciones controlado por software desde el empotrado. Es un elemento importante para prevenir los cuelgues del router ya que este sistema queda embarcado en un autocar de forma permanente. 

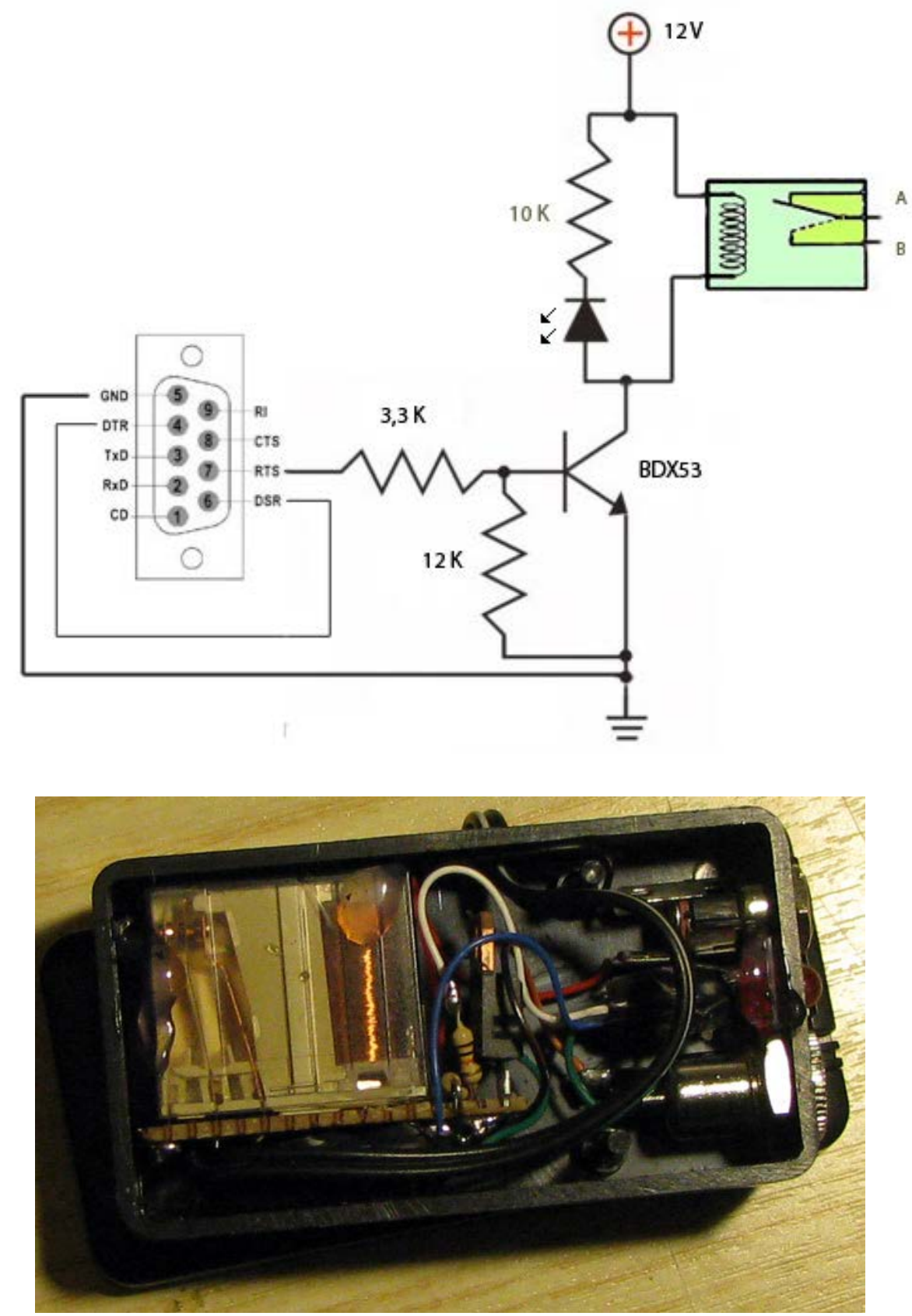

Figura 37. Circuito de activación/desactivación de alimentación del router a través de puerto RS232.

El resto del hardware utilizado son conectados a través de puertos USB y tampoco requiere alimentación adicional a la suministrada por este puerto $(5 \mathrm{Vdc})$ : un dispositivo Bluetooth y un módulo GPS.

Las especificaciones del Bluetooth son:

- Obediente con la especificación de Bluetooth V2.0.

- Operación de la clase 1. 
- $\quad$ Interfaz del USB de la velocidad completa, obediente con USB 2.0.

- Indicación del LED.

- Software aumentable.

- Antena incorporada.

- Banda de frecuencia: venda del ISMO 2.4GHz.

- Tasa de datos máxima: 3Mbps.

- Sensibilidad: -89dBM en 0.1\% AZUFAIFAS.

- Rango de operación del 100M.

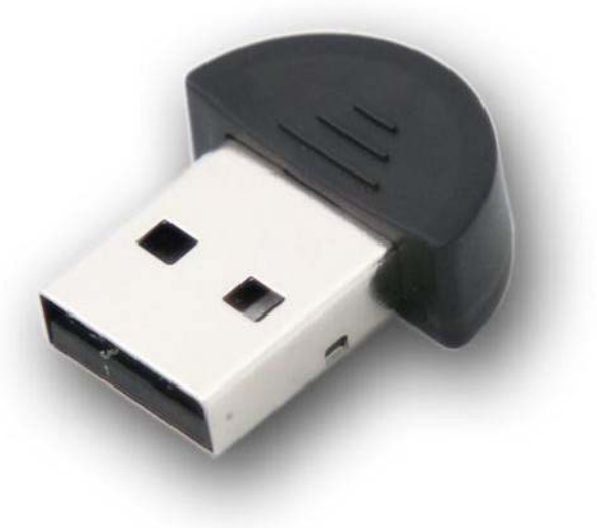

Figura 38. Dispositivo Bluetooth.

El módulo GPS tiene las siguientes características:

- Totalmente estanco.

- Una única conexión USB.

- Emula un puerto serie RS-232.

- Formato de salida: NMEA-0183 v2.20 a 4800 baud 8N1.

- Mensajes NMEA Soportados: GGA, GSA, GSV, RMC, GLL y VTG.

- Longitud del cable: 2 metros.

- Sujeción: Magnética.

- Compatible con sistemas Unix. 


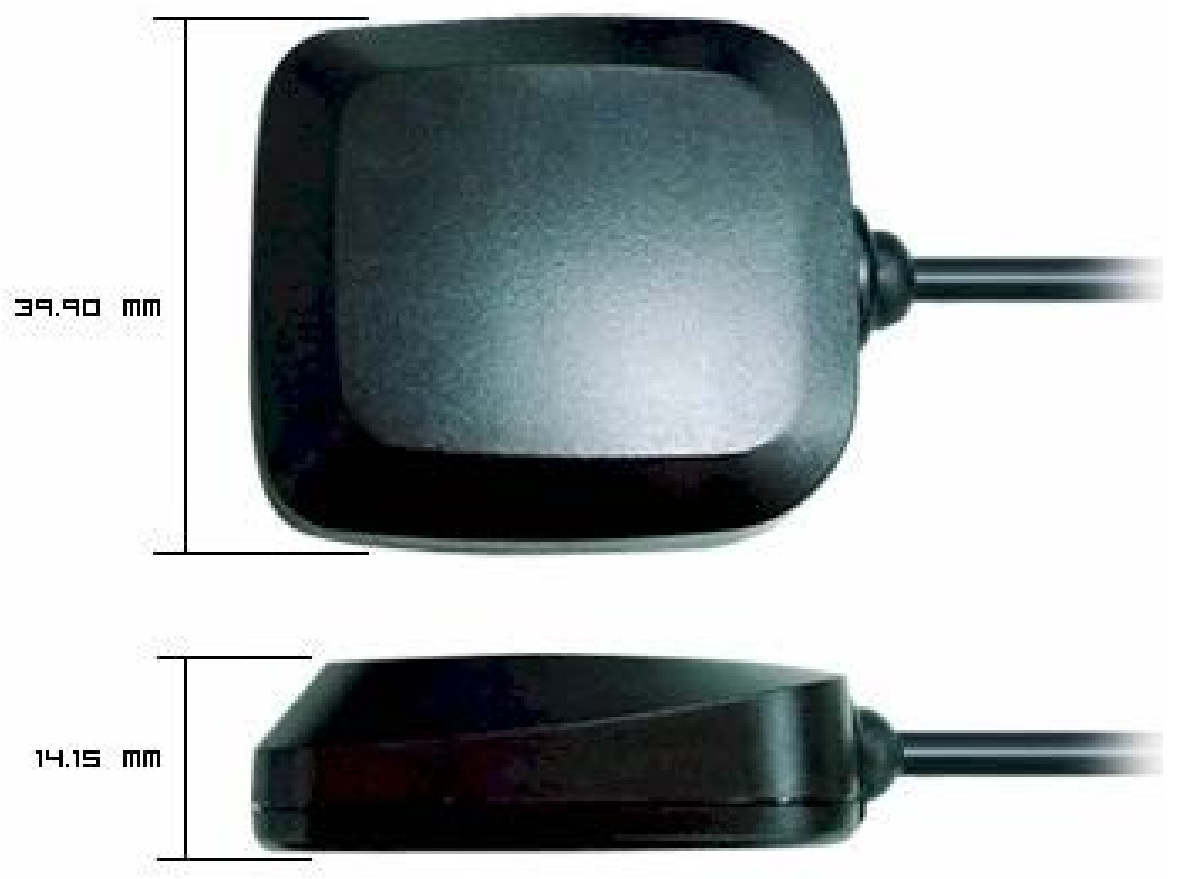

Figura 39. Módulo GPS.

A modo de resumen, podemos decir que el sistema embebido es conectado directamente a la tensión auxiliar del autocar $(12 \mathrm{Vdc})$. A éste se le conecta: por USB el GPS y el Bluetooth, y por Ethernet el router que, a su vez, es alimentado por los 5 Vdc de otro de los puertos USB. El cable de alimentación del router pasa por el circuito de desconexión controlada por el puerto RS232 del empotrado. El circuito de desconexión mediante relé (contacto normalmente cerrado) es alimentado a través del cable amarillo (12Vdc) de la fuente de alimentación del sistema embebido.

Respecto al software, se han desarrollado dos ejecutables en lenguaje C: uno encargado llevar a cabo el sondeo periódico de dispositivos Bluetooth y generación de ficheros, y otro encargado de detectar la llegada a una parada a través de GPS. El primero de ellos, llamado "Cliente", consulta periódicamente un fichero que recoge el identificador de la última parada registrada por el segundo, llamado "Paradas".

También se han desarrollado numerosos scripts con ejecución periódica mediante CRON para tareas de obtención de datos del GPS, empaquetado y envío de información a servidores externos, comprobación de funciones de los procesos y conectividad a Internet, etc.

Uno de los principales aspectos clave a la hora de preparar la parte software del prototipo ha sido el de utilizar el sistema de archivos BTRFS sobre el que instalar 
el sistema operativo Ubuntu 11.04. Este sistema se comporta mejor a la hora soportar apagones bruscos.

Para describir de forma global el proceso de obtención de datos, se presenta un ejemplo gráfico en el que un autocar va desde la parada número 1 a la parada número 2, situadas 100 metros la una de la otra. Se asume que el tramo comienza en el instante 0 segundos y finaliza en el instante 20 segundos. El margen o radio de detección de parada es de 10 metros y el tiempo entre escaneos BT es de 4 segundos.

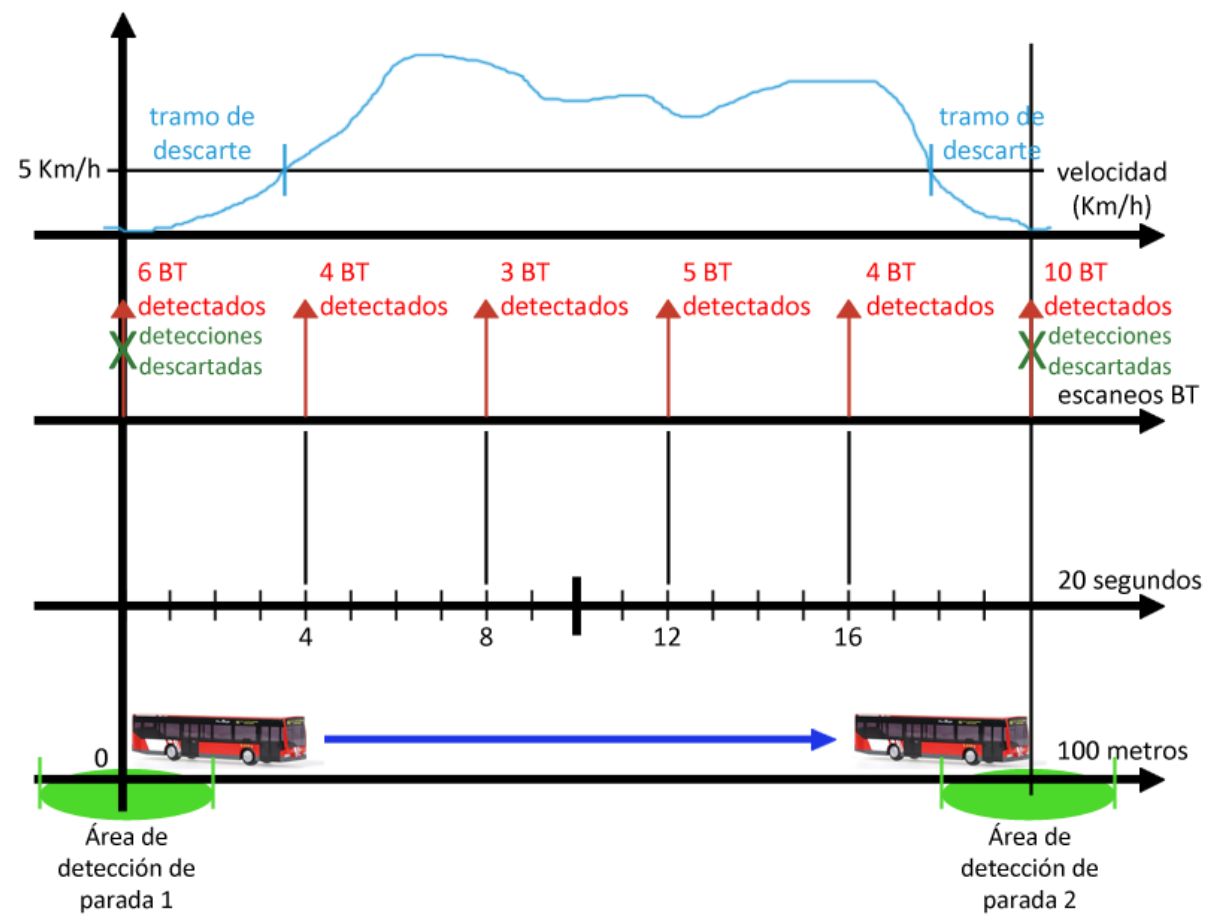

Figura 40. Ejemplo de proceso de recopilación de datos.

Como se puede observar, las detecciones realizadas en los instantes 0 y 20 segundos, son descartadas en el preproceso debido que en esos instantes:

- El autocar no había salido de la zona de detección de parada (sombra verde).

- El autocar no había superado la velocidad mínima de $5 \mathrm{Km} / \mathrm{h}$.

Son muchas las posibles circunstancias que pueden darse pero, tras semanas de pruebas, las consideraciones descritas anteriormente han sido suficientes para esta parte del pre procesado. 


\subsection{Matrices Origen-Destino}

El conocimiento adquirido por las encuestas no sería suficientemente útil si no hubiera una cuantificación ordenada de los datos recopilados. Una de las maneras más utilizadas para representar los datos de las encuestas sobre el flujo de viajeros de una determinada línea de autobús o metro es representarlas con una matriz cuadrada en la que el término de la fila y la columna j indica la cantidad de pasajeros que, habiendo entrado en la estación o parada $y$, salen por la estación o parada j. Por este motivo estas matrices se llaman matrices origen-destino o simplemente matrices OD. En [Dawei'2008] se comenta que las probabilidades de entrar en una parada y salir en otra permanecen más estables en el tiempo que el número concreto de pasajeros que lo hacen. Dicho de otra manera, los términos absolutos son poco indicativos para un momento determinado, por lo que habitualmente los datos se presentan normalizadas, es decir, los términos se dividen por el número total de pasajeros, de forma que el término ij de la matriz indica la probabilidad de que un pasajero que ha entrado en la parada o estación y salga por la j.

Aunque tradicionalmente, como se ha dicho, las matrices OD han encontrado mediante encuestas, su elevado precio y la poca rapidez para obtenerlas han hecho conveniente encontrar otros métodos que permitan llegar a este conocimiento acortando el tiempo dedicado. Tener los resultados cuanto antes se ha convertido en una cuestión crítica en determinados casos, como, por ejemplo, en el transporte urbano con autobuses. Esto sucede no sólo porque en este entorno las matrices OD raramente son invariantes con el tiempo, sino porque la toma de decisiones de las administraciones locales sobre las estructuras o el diseño de las líneas de autobús puede ser también más rápida que en el caso de las infraestructuras ferroviarias o de carreteras, la previsión de las cuales, por su complejidad intrínseca, no puede ser $\tan$ adaptativa.

Muchos autores han propuesto en los últimos años varios métodos que intentan minimizar tanto el coste como el tiempo de obtención de las matrices OD. Una forma de reducir el coste de recogida de los datos es sustituir las encuestas o el recuento manual por una recopilación de los datos obtenidos a partir de los sistemas ADC (Automatic Data Collection), como, por ejemplo, los de venta automática de 
billetes. El problema de estos sistemas, para el caso del transporte con autobuses, es que no suelen obtener datos del momento en que baja el pasajero ya veces tampoco el momento en que entra. Hay autores, como por ejemplo [Wang'2011], que intentan obtener los datos de las subidas de pasajeros correlacionando temporalmente los datos recogidos por las tarjetas de pago y los datos de ubicación del vehículo en el momento en que se hace el pago (cuando entra el pasajero al autobús). El sistema no proporciona datos de las bajadas de los pasajeros. En [Zhao'2007] se explica, sin embargo, cómo obtener de forma aproximada una matriz OD sólo con los datos de subida de los pasajeros. Para conseguirlo, se considera que los viajeros hacen a lo largo del día una cadena de transbordos que los llevan de nuevo al punto de origen al final de la jornada.

Así, los datos de subida de unos pasajeros por la mañana permiten estimar sus datos de salida después del último transbordo. El método, por tanto, obliga a dos hipótesis muy razonables: la primera, que los pasajeros terminan el viaje donde el empiezan y, la segunda, que los transbordos los hacen sólo caminando y que la distancia no es demasiado grande.

Desgraciadamente, estas hipótesis, tal como se comenta en [Kostakos'2010], pueden ser demasiado restrictivas en el caso de estudiar una red sólo de autobuses.

Actualmente se han desarrollado comercialmente sistemas de recuento automático de pasajeros basados en diversas tecnologías, como la detección de cabezas con cámaras de video vigilancia [Boon'2007] o mediante sensores de rayos infrarrojos, pero tienen un precio demasiado elevado para las administraciones locales, además de problemas de detección precisa en determinadas circunstancias (ver [Kostakos'2010]). Además, muchos de estos sistemas permiten saber cuántos pasajeros entran en una parada y cuantos salen, pero eso no es suficiente para averiguar la matriz OD, dado que sólo tendríamos como datos las sumas de los elementos de cada columna y cada fila.

En este contexto diversos autores han intentado hacer uso de la telefonía móvil y de su extendida implantación para obtener datos de los flujos de personas en el transporte. Por ejemplo, en [Caceres'2007], para estudiar el flujo de coches en autopistas, se plantea utilizar los datos de registro de los teléfonos de los ocupantes que recogen las operadoras en sus células GSM. Básicamente lo que se propone es 
llegar a cuantificar el flujo de vehículos correlato la ocupación media con la cantidad de teléfonos registrados. Naturalmente, este método no es aplicable al caso los autobuses, porque la ocupación de cada vehículo abarca un intervalo mucho mayor de pasajeros que en un turismo, aparte de que la precisión de una célula GSM (sobre todo en áreas rurales) es muy baja y el sistema dependería de la cesión de los datos por parte de las operadoras. En cualquier caso, no se plantea el uso de la característica de conectividad Bluetooth de los teléfonos móviles, a diferencia de Kostakos y otros [Kostakos'2010], los que sí utilizan esta posibilidad para autobuses, resultando un sistema de obtención de matrices OD que divide por 20 el coste de sistemas de recuento de personas ([Kostakos'2010], p. 1799) y que, por tanto, hacen más fácilmente extensible el sistema a toda una flota.

En [Montero'2012] también se utilizan datos de las conexiones Bluetooth de los pasajeros para hacer una estimación de las matrices OD variantes con el tiempo. Se comenta que las matrices OD no son observables directamente y lo que se hace es ajustar una matriz semilla obtenida habitualmente por encuestas con los datos de conectividad Bluetooth observadas.

\subsection{Escenario de capturas: Prueba piloto}

La Universidad Politécnica de Cataluña y la Universidad Politécnica de Cartagena han desarrollado un sistema de estimación de las matrices OD fundamentado en la conectividad Bluetooth de los terminales móviles. Concretamente, se ha puesto en práctica un sistema en un autobús de Barcelona con el objetivo de obtener una matriz OD de los pasajeros con Bluetooth activo. Estos tipos de sistemas se basan habitualmente en el hecho de que un dispositivo Bluetooth puesto en el modo descubrible ha de responder a una demanda de descubrimiento de otro dispositivo enviando 18 caracteres hexadecimales (12 que son un identificador único para cada dispositivo existente y 6 que indican el tipo de dispositivo) (ver [IEEE'2005]). El núcleo del sistema se encarga del escaneado periódicamente. Naturalmente, para obtener una indicación de lo que podría ser la matriz OD, los datos de los dispositivos Bluetooth deben ser correlados con la posición del vehículo en el momento de la detección. Esto se consigue con un dispositivo GPS que en varios momentos transfiriendo a la memoria persistente del sistema la posición y el tiempo, por lo que puede obtenerse para cada dispositivo 
descubierto la parada origen y la parada destino. Lógicamente, los datos obtenidos se deben procesar de alguna manera, con el fin de rechazar las espurias, debidas, por ejemplo, a otros vehículos que durante un tramo van paralelos al autobús. El objetivo básico era filtrar la matriz OD de dispositivos Bluetooth activos así obtenida y dejarla sin datos erróneos.

Obtener una matriz OD relativa sólo a los pasajeros con terminales con Bluetooth activo sería satisfactorio si todos los pasajeros llevaran un terminal móvil en estas condiciones, pero la realidad es bien diferente. En efecto, el Instituto Nacional de Tecnologías de la Comunicación, dependiendo del Ministerio de Industria, Energía y Turismo, señala en un estudio de 2012 [Perez'2012] que alrededor del 50\% de los usuarios de telefonía móvil tiene un Smartphone y que de éstos, el 98\% dispone de conectividad Bluetooth y un 89\% de Wi-Fi. El dato más importante, sin embargo, es que el 18\% siempre tienen el dispositivo Bluetooth en modo descubrible, mientras que el $61 \%$ sólo lo conecta cuando va a hacer OS 4 . Por lo tanto, podemos esperar que el alrededor de un 9\% de los pasajeros que entran en un autobús lleven un teléfono móvil con Bluetooth en modo descubrible y, por tanto, en condiciones de ser detectado por el sistema desarrollado.

\subsection{Modelado del conjunto de terminales Bluetooth activos}

Llegado este punto debemos plantearnos si la matriz OD obtenida sólo con los datos de Bluetooth es extrapolable a la totalidad de los pasajeros y si, como consecuencia, el sistema implementado puede servir para obtener la matriz OD global de todos los pasajeros, lleven o no teléfono móvil. Para hacer esto, se ha elaborado un estudio teórico [Albarracin'2014] que permitirá evaluar el error que supondría considerar que la matriz OD derivada sólo de los datos de conectividad Bluetooth es la matriz OD global, así como los parámetros de los que depende este error y qué valores deberían tener para minimizarlo. Las conclusiones a que se llegue deberán permitir decidir si el sistema implementado puede ser una buena herramienta automática para encontrar matrices OD en líneas de autobuses. Además, todo el estudio será también válido en el caso de considerar dispositivos WiFi en lugar de dispositivos Bluetooth. 


\subsubsection{Propiedades de la variable aleatoria de Poisson}

La función de densidad de probabilidad de una variable aleatoria de Poisson de parámetro $\lambda$ viene dada por

$$
f_{X}(k ; \lambda)=\frac{e^{-\lambda} \cdot \lambda^{k}}{k !}
$$

La esperanza y la varianza de una variable aleatoria de este tipo son, respectivamente:

$$
\begin{gathered}
E(X)=\lambda \\
\sigma_{X}^{2} \equiv E(X-E(X))^{2}=\lambda
\end{gathered}
$$

Consideramos ahora la suma de $n$ variables aleatorias de Poisson $X_{i}$ independientes de parámetro $\lambda_{i}$. Se puede ver fácilmente que la v.a. $Y=\sum_{n} X_{i}$ es también una v.a. de Poisson y su esperanza y varianza vienen dadas por:

$$
E(Y)=\sigma^{2}(Y)=E\left(\sum_{n} X_{i}\right)=\sum_{n} E\left(X_{i}\right)=\sum_{n} \lambda_{i}
$$

Como consecuencia inmediata, si en vez de sumar $n$ v.a. de Poisson, obtenemos la media de $n$ v.a. de Poisson de parámetro $\lambda$, igual en todos los casos, tenemos

$$
Y=\frac{1}{n} \sum_{n} X_{i} \Rightarrow E(Y)=\frac{1}{n} \sum_{n} \lambda=\lambda
$$

En general, como que $\sigma_{X}^{2} \equiv E(X-E(X))^{2}=E\left(X^{2}\right)-E^{2}(X)$ 


$$
\sigma_{k X}^{2}=E\left((k X)^{2}\right)-E^{2}(k X)=E\left(k^{2} X^{2}\right)-(k E(X))^{2}=k^{2} E\left(X^{2}\right)-k^{2} E^{2}(X)=k^{2} \sigma_{X}^{2}
$$

y, como que, por otra parte, $\sigma_{X \pm Y}^{2}=\sigma_{X}^{2}+\sigma_{Y}^{2}$, vemos que, siendo $Y=\frac{1}{n} \sum_{n} X_{i}$ la suma de $n$ v.a. de Poisson independientes de parámetro $\lambda$, se cumple

$$
\sigma_{Y}^{2}=\frac{1}{n^{2}} \cdot \sigma_{\sum x}^{2}=\frac{1}{n^{2}}(n \cdot \lambda)=\frac{\lambda}{n}
$$

o escrito de otra manera, si $h \geq 0$

$$
E(h \cdot \lambda-h \cdot X)^{2}=h^{2} \cdot \lambda
$$

Para estudiar el subconjunto de persones que tienen activa la conexión bluetooth de su móvil, lo modelaremos como un sistema donde entra una realización proveniente de una va. de Poisson y donde cada uno de estos elementos saldrán o no del sistema con una probabilidad $p$ y 1- $p$ respectivamente. Trataremos de ver que distribución podemos esperar a la salida (fig. 32)

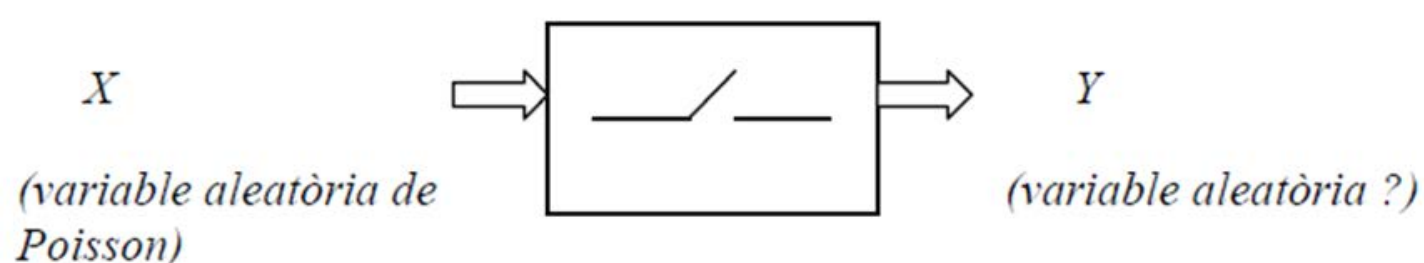

(prob. $p$ )

Figura 41. Modelo del sistema

Por hipótesis,

$$
P\{X=n\}=e^{-\lambda} \cdot \frac{\lambda^{n}}{n !} \quad n=0,1,2, \ldots
$$




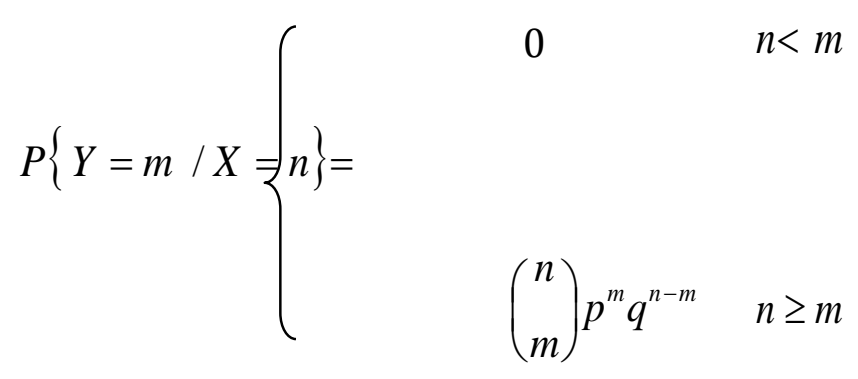

Por tanto,

$$
P\{Y=m\}=\sum_{n=0}^{\infty} P\{Y=m / X=n\} \cdot P\{X=n\}=\sum_{n=m}^{\infty}\left(\begin{array}{l}
n \\
m
\end{array}\right) p^{m} q^{n-m} e^{-\lambda} \frac{\lambda^{n}}{n !}=
$$

$\sum_{n=m}^{\infty}\left(\begin{array}{l}n \\ m\end{array}\right) p^{m} q^{n-m} e^{-\lambda} \frac{\lambda^{n}}{n !}=\frac{e^{-\lambda} p^{m}}{m !} \sum_{n=m}^{\infty} \frac{n !}{(n-m)^{!}} q^{n-m} \frac{\lambda^{n}}{n !} \quad$, y multiplicando por $\lambda^{m} \cdot \lambda^{-m}=1$

tenemos,

$$
P\{Y=m\}=e^{-\lambda} \cdot \frac{(\lambda \cdot p)^{m}}{m !} \sum_{n=m}^{\infty} \frac{n !}{(n-m) !} \frac{(\lambda \cdot q)^{n-m}}{n !}=e^{-\lambda} \cdot \frac{(\lambda \cdot p)^{m}}{m !} \sum_{i=0}^{\infty} \frac{(\lambda \cdot q)^{i}}{i !}
$$

El sumatorio de la expresión anterior se corresponde con el desarrollo en serie de Taylor de la exponencial:

$$
P\{Y=m\}=e^{-\lambda} \cdot \frac{(\lambda \cdot p)^{m}}{m !} e^{\lambda q}, \quad \text { y recordando que } q=1-p, \text { obtenemos }
$$

finalmente 


$$
P\{Y=m\}=e^{-\lambda p} \cdot \frac{(\lambda \cdot p)^{m}}{m !} \quad m=0,1,2, \ldots
$$

que se corresponde con una distribución de Poisson de parámetro $\lambda \cdot p$

\subsubsection{Aplicación a les matrices OD}

Por definición, una matriz $M=\left(m_{i j}\right)$ puede ser una matriz OD si

$$
m_{i j}=0 \quad \forall i \geq j
$$

(7)

$$
\sum_{i, j} m_{i j}=1
$$

Sea $k$ el número medio de pasajeros por trayecto. Estimaremos la matriz $k \cdot M$ para una realización $P=\left(X_{i j}\right)$, donde cada $X_{i j}$ sea una distribución de Poisson de parámetro $k \cdot m_{i j}$.

Considerando tota la matriz $P$, la esperanza será $E(P)=\left(E\left(x_{i j}\right)\right)=\left(k \cdot m_{i j}\right)=k \cdot\left(m_{i j}\right)$ i

El error de esta estimación vendrá dado por

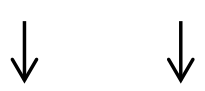

$$
E\left(\|k M-P\|^{2}\right) \equiv E\left(\sum_{i, j}\left(k m_{i j}-x_{i j}\right)^{2}\right)=\sum_{i, j} E\left(k m_{i j}-x_{i j}\right)^{2}=\sum_{i, j} k m_{i j}=k \sum_{i, j} m_{i j}=k
$$


Nosotros estimamos, de hecho, $M$ y no $k M$, es decir, estimamos la matriz normalizada, pere el error se deduce fácilmente como consecuencia:

$$
\begin{aligned}
& E\left(\left\|M-\frac{1}{k} P\right\|^{2}\right)=E\left(\left\|\frac{1}{k}(k M-P)\right\|^{2}\right)=E\left(\frac{1}{k^{2}}\|k M-P\|^{2}\right)= \\
&(8) \\
& \downarrow \\
&=\frac{1}{k^{2}} E\left(\|k M-P\|^{2}\right)=\frac{1}{k^{2}} \cdot k=\frac{1}{k}
\end{aligned}
$$

En vez de una realización, sin embargo, hacemos la media de $n$ realizaciones, es decir, en vez de la matriz $(P)$ tendremos $\left(P_{n}\right)=\frac{1}{n} \sum_{i=1}^{n}\left(P_{i}\right)$ y, por tanto, el error será

$$
E\left(\left\|k M-P_{n}\right\|^{2}\right)=E\left(\sum_{i, j}\left(k \cdot m_{i j}-\frac{\sum_{l} x_{i j, l}}{n}\right)^{2}=\sum_{i, j} E\left(k \cdot m_{i j}-\frac{\sum_{l} x_{i j, l}}{n}\right)^{2}\right.
$$

Teniendo en cuenta que $\frac{\sum_{l} x_{i j, l}}{n}$ es una media de variables aleatorias de Poisson y aplicando (4), podemos escribir

$$
E\left(\left\|k M-P_{n}\right\|^{2}\right)=\frac{1}{n} \sum_{i, j} k \cdot m_{i j}=\frac{1}{n} \cdot k \sum_{i, j} m_{i j}=\frac{k}{n}
$$

y de aquí, de forma análoga a como hicimos en (9), podemos deducir que 
(10)

$$
E\left(\left\|M-\frac{1}{k} P_{n}\right\|^{2}\right)=E\left(\left\|\frac{1}{k}\left(k M-P_{n}\right)\right\|^{2}\right)=\frac{1}{k^{2}} E\left(\left\|k M-P_{n}\right\|^{2}\right)=\frac{1}{k^{2}} \cdot \frac{k}{n}=\frac{1}{k n}
$$

A continuación tendremos en cuenta para hacer la estimación de la matriz OD sólo la parte de los pasajeros que tienen activo el sistema bluetooth de su móvil. Considerando que la probabilidad de tener el bluetooth activo es uniforme y igual a $p$, si llamamos $B=\left(b_{i j}\right)$ a la matriz de los elementos de la cual son el número de pasajeros que tienen el bluetooth activo entre cada par de paradas, sabemos per (6) que cada $b_{i j}$ será una v.a. de Poisson de parámetro

$$
\lambda_{b}=p \cdot k \cdot m_{i j}
$$

Por tanto, el error que cometemos al estimar la matriz $M$ con la matriz $B$, viene dado, según (9), por

$$
E\left(\left\|M-\frac{1}{k p} B\right\|^{2}\right)=\frac{1}{k p}
$$

y cuando hacemos la media de las diferentes matrices $B_{i}$, según (11), el error será

$$
E\left(\left\|M-\frac{1}{k p n} \sum_{i=1}^{n} B_{i}\right\|^{2}\right)=\frac{1}{k p n}
$$


En realidad, en la simulación se aproxima en (13) el término $\frac{1}{k p} B$ por $\frac{B}{\sum_{s, t} b_{s, t}}$ , per la qual cosa debemos plantearnos averiguar cómo será el error que esperamos tener y que vendrá dado, en el caso de considerar un único trayecto, por

$$
\varepsilon_{\text {esp }}=E\left(\left\|M-\frac{B}{\sum_{s, t} b_{s, t}}\right\|^{2}\right)
$$

Para verlo, tendremos en cuenta la equivalencia aproximada [Mood'1974]

$$
E\left[\frac{X}{Y}\right] \approx \frac{E[X]}{E[Y]} \cdot\left(1+\frac{\sigma^{2}(Y)}{(E(Y))^{2}}\right)-\frac{\operatorname{Cov}[X, Y]}{(E(Y))^{2}}
$$

donde

$$
\operatorname{Cov}[X, Y]=E[X, Y]-E[X] \cdot E[Y]
$$

Estudiamos ahora la expresión $\quad E\left[\frac{b_{i j}}{\sum_{s, t} b_{s t}}\right]$. Podremos escribir, según (16),

$$
E\left[\frac{b_{i j}}{\sum_{s, t} b_{s t}}\right] \approx \frac{E\left(b_{i j}\right)}{E\left(\sum_{s, t} b_{s t}\right)} \cdot\left(1+\frac{\sigma^{2}\left(\sum_{s, t} b_{s t}\right)}{\left[E\left(\sum_{s, t} b_{s t}\right)\right]^{2}}\right)-\frac{\operatorname{Cov}\left[b_{i j}, \sum_{s, t} b_{s t}\right]}{\left[E\left(\sum_{s, t} b_{s t}\right)\right]^{2}}
$$

Sin embargo, de acuerdo con (3), (7) y (12), si suponemos que las v.a. $b_{\mathrm{ij}}$ son independientes, podemos escribir

$$
E\left(b_{i j}\right)=p k m_{i j}
$$




$$
\begin{aligned}
& E\left(\sum_{s, t} b_{s t}\right)=p k \sum_{i, j} m_{i j}=p k \\
& \sigma^{2}\left(\sum_{s, t} b_{s t}\right)=E\left(\sum_{s, t} b_{s t}\right)=p k \\
& \operatorname{Cov}\left(b_{i j}, \sum_{s, t} b_{s t}\right)=\sum_{s, t} \operatorname{Cov}\left(b_{i j}, b_{s t}\right)
\end{aligned}
$$

No obstante, $\sum_{s, t} \operatorname{Cov}\left(b_{i j}, b_{s t}\right)=\sum_{s, t}\left\{E\left(b_{i j}, b_{s t}\right)-E\left(b_{i j}\right) \cdot E\left(b_{s t}\right)\right\}$

y si los $b_{i j}$ son independientes entre sí, como hemos supuesto,

$E\left(b_{i j}, b_{s t}\right)=E\left(b_{i j}\right) \cdot E\left(b_{s t}\right) \quad($ si $i j \neq s t)$ i $\quad E\left(b_{i j}, b_{i j}\right)-E\left(b_{i j}\right) \cdot E\left(b_{i j}\right) \equiv \sigma^{2}\left(b_{i j}\right)$ (para el caso $i j=s t$ ), podremos escribir

$$
\sum_{s, t} \operatorname{Cov}\left(b_{i j}, b_{s t}\right)=\sum_{s, t}\left\{E\left(b_{i j}, b_{s t}\right)-E\left(b_{i j}\right) \cdot E\left(b_{s t}\right)\right\}=\sigma^{2}\left(b_{i j}\right)=E\left(b_{i j}\right)=p \cdot k \cdot m_{i j}
$$

Así pues, substituyendo (19)-(22) en (18),

$$
E\left[\frac{b_{i j}}{\sum_{s, t} b_{s t}}\right] \approx \frac{p \cdot k \cdot m_{i j}}{p \cdot k} \cdot\left(1+\frac{p \cdot k}{(p \cdot k)^{2}}\right)-\frac{p \cdot k \cdot m_{i j}}{(p \cdot k)^{2}}=m_{i j}
$$

Podemos hacer un desarrollo parecido para la varianza. La aproximación equivalente a (16) es [Mood'1974]

$$
\sigma^{2}\left[\frac{X}{Y}\right] \approx\left[\frac{E[X]}{E[Y]}\right]^{2} \cdot\left(\frac{\sigma^{2}(X)}{(E(X))^{2}}+\frac{\sigma^{2}(Y)}{(E(Y))^{2}}-\frac{2 \cdot \operatorname{Cov}(X, Y)}{E(X) \cdot E(Y)}\right)
$$


y en nuestro caso, teniendo en cuenta (19)-(24)

$$
\begin{aligned}
E\left[m_{i j}-\frac{b_{i j}}{\sum_{s, t} b_{s t}}\right)^{2} \approx E\left\{\left[\frac{b_{i j}}{\sum_{s, t} b_{s t}}-E\left[\frac{b_{i j}}{\sum_{s, t} b_{s t}}\right]\right]^{2}\right\} \equiv \sigma^{2}\left[\frac{b_{i j}}{\sum_{s, t} b_{s t}}\right] \approx \\
\approx\left[\frac{E\left[b_{i j}\right]}{E\left[\sum_{s, t} b_{s t}\right]}\right]^{2} \cdot\left(\frac{\sigma^{2}\left(b_{i j}\right)}{\left(E\left(b_{i j}\right)\right)^{2}}+\frac{\sigma^{2}\left(\sum_{s, t} b_{s t}\right)}{\left(E\left(\sum_{s, t} b_{s t}\right)\right)^{2}}-\frac{2 \cdot \operatorname{Cov}\left(b_{i j}, \sum_{s, t} b_{s t}\right)}{E\left(b_{i j}\right) \cdot E\left(\sum_{s, t} b_{s t}\right)}\right)= \\
=\frac{\left(p \cdot k \cdot m_{i j}\right)^{2}}{(p \cdot k)^{2}} \cdot\left(\frac{p \cdot k \cdot m_{i j}}{\left(p \cdot k \cdot m_{i j}\right)^{2}}+\frac{p \cdot k}{(p \cdot k)^{2}}-\frac{2 p \cdot k \cdot m_{i j}}{p \cdot k \cdot m_{i j} \cdot p \cdot k}\right)=m_{i j}^{2}\left(\frac{1}{p k m_{i j}}+\frac{1}{p k}-\frac{2}{p k}\right)
\end{aligned}
$$

Es decir,

$$
E\left(m_{i j}-\frac{b_{i j}}{\sum_{s, t} b_{s t}}\right)^{2}=\frac{1}{p k}\left(m_{i j}-m_{i j}^{2}\right)
$$

Así pues, volviendo a (15) y teniendo en cuenta (25)

$$
\begin{aligned}
& \varepsilon_{\text {esp }}=E\left(\left\|M-\frac{B}{\sum_{s, t} b_{s, t}}\right\|^{2}\right)=E\left\{\sum_{i, j}\left(m_{i j}-\frac{b_{i j}}{\sum_{s, t} b_{s t}}\right)^{2}\right\}=\sum_{i, j} E\left(m_{i j}-\frac{b_{i j}}{\sum_{s, t} b_{s t}}\right)^{2}= \\
& =\sum_{i, j}\left[\frac{1}{p k}\left(m_{i j}-m_{i j}^{2}\right)\right]=\frac{1}{p k}\left(\sum_{i, j} m_{i j}-\sum_{i, j} m_{i j}^{2}\right)=\frac{1}{p k}\left(1-\|M\|^{2}\right)
\end{aligned}
$$


Por tanto, según (7), podemos afirmar que el error de nuestra aproximación será

$$
\varepsilon_{\text {esp }} \approx \frac{1}{p k}\left(1-\|M\|^{2}\right)<\frac{1}{p k}
$$

y, en consecuencia, la simulación será más precisa cuanto mayor sea p y k, es decir, cuanto mayor sea el número medio de pasajeros y el porcentaje de personas con Bluetooth activo.

Para n muestras el error se escribiría así

$$
\varepsilon_{\text {esp }} \approx \frac{1}{n p k}\left(1-\|M\|^{2}\right)<\frac{1}{n p k}
$$

Los resultados de las simulaciones en [Alabarracin'2014] muestran que incluso con un porcentaje de BT activos del 5\% es posible predecir matrices OD con el método expuesto con errores inferiores al 5\% de la norma de la matriz base, siempre que el número de trayectos iguales estudiados sea suficientemente grande. En cualquier caso, el error disminuye si el porcentaje de terminales con BT activos aumenta, pero un porcentaje en torno a este $5 \%$ es esperable, como se ha explicado, por las costumbres actuales de la población. En conclusión este estudio demuestra que los sistemas desarrollados de adquisición y filtrado expuestos inicialmente y el sistema de simulación que se explica a continuación son herramientas válidas de cara al cumplimiento de los objetivos y alcance previstos en este trabajo de tesis y que se describieron en el capítulo primero.

\subsection{Desarrollo del generador de matrices O-D}

El software de matrices O-D para sistemas de transporte público (SIMOD) se nutre de la información proporcionada por una estación de detección que incorpora el sistema de adquisición descrito en el capítulo 2. Ésta va empotrada dentro del autobús y será la encargada de capturar los registros y almacenarlos en ficheros de texto plano (*.txt) para su tratamiento. La información del fichero que almacenaremos se genera durante un trayecto parada-origen y parada-destino, 
recogiendo las detecciones de los bluetooth de las personas que viajan en el autocar en el trayecto que va desde una parada origen hasta una parada destino. También incluye datos adicionales de la línea y del trayecto.

Estos archivos que poseen una estructura especial serán tratados mediante una aplicación que será desarrollada en Php para re-estructurar la información almacenándola en una base de datos MySql, la cual se filtra de acuerdo a una serie de requisitos que serán explicados posteriormente.

Las distintas tecnologías que han sido utilizadas se resumen brevemente a continuación:

- App Server: es un paquete de software que instala de forma automática lo necesario para el desarrollo Web, muy útil y sobe todo práctico.

- PHP: Se ha elegido este lenguaje de programación para el desarrollo de esta aplicación porque es un lenguaje de programación de uso general de código del lado del servidor originalmente diseñado para el desarrollo web de contenido dinámico. Elegido por su rapidez, versatilidad y eficiencia, permite la conexión a diferentes tipos de servidores de bases de datos tales como MySQL (gestor de base de datos que utilizaremos), PostgreSQL, Oracle, ODBC, DB2, Microsoft SQL Server, Firebird y SQLite.

- MySql: Se seleccionó este gestor de base de datos porque es un software de código abierto, licenciado bajo la GPL de la GNU, además de por ofrecer velocidad al realizar las operaciones, lo que le hace uno de los gestores con mejor rendimiento. Otra característica es su bajo costo en requerimientos para la elaboración de bases de datos, ya que debido a su bajo consumo puede ser ejecutado en una máquina con escasos recursos sin ningún problema.

- FTP: Protocolo escogido para la transmisión de los archivos que se van descargando desde el cliente FTP por su velocidad y por la independencia del sistema operativo de cada equipo.

- Dreamweaver: Seleccionado porque es una herramienta destinada a la construcción, diseño y edición de sitios, videos y aplicaciones Web basados en estándares. Actualmente es uno de los programas más 
utilizados en el sector del diseño y la programación web, por sus funcionalidades, su integración con otras herramientas

\subsubsection{Arquitectura del software}

La información proporcionada consta de ficheros en texto plano generados por el sistema de adquisición Bluetooth, que obtiene una serie de parámetros que se encuentran organizados de la siguiente manera figura 42.

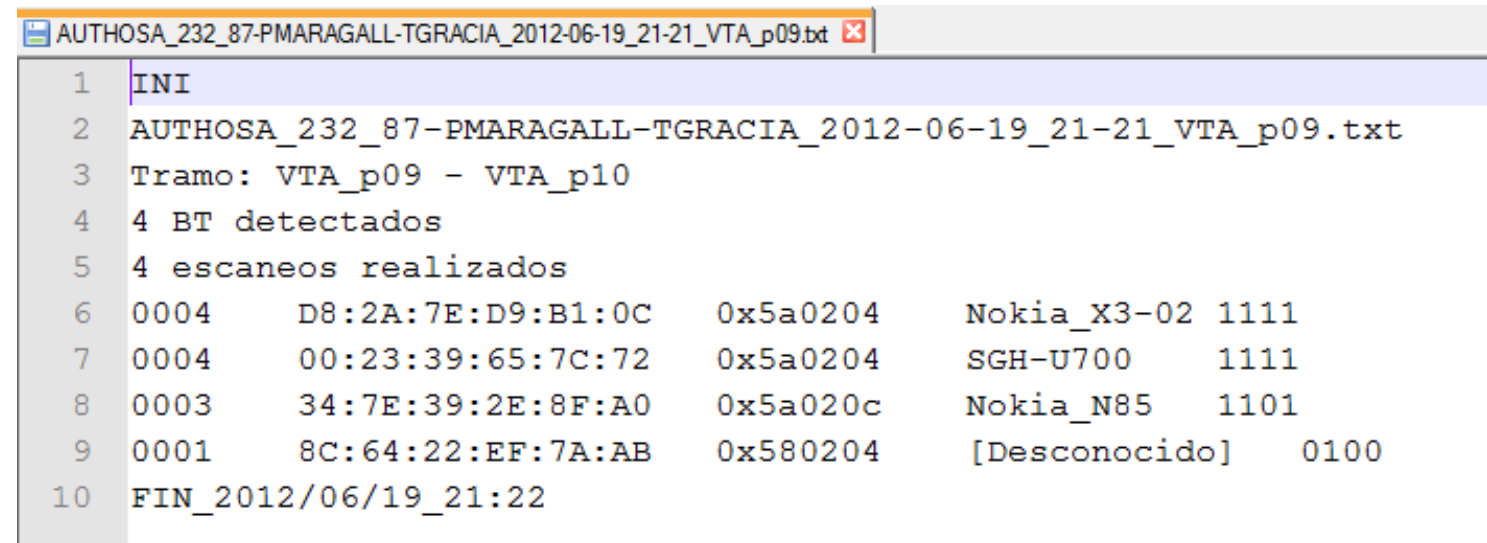

Figura 42. Archivo de texto plano generado por el dispositivo Bluetooth incrustado en el autobús.

Como se puede apreciar en la figura 42, el archivo de texto plano contiene toda la información que necesitamos y tenemos como objetivo organizarla.

Se han planteado una serie de diagramas de procesos que serán explicados a continuación. Primero partiremos del diagrama general (ver Diagrama 1) en el que se detalla de forma general las actividades a realizarse en el desarrollo.

Como se aprecia en el Diagrama 1, la primera parte consiste en obtener la información en texto plano como ya se ha comentado en ocasiones anteriores. Una vez obtenida la información se analizan los archivos partiendo de la premisa que todos tienen la misma estructura (ver figura 43), porque han sido almacenados basándose a una estructura determinada, que proporciona como resultado final los ficheros de texto que serán analizados.

En el Diagrama 2 se detalla el proceso seguido a la hora de recorrer cada fichero, identificando la información relevante y necesaria para ser almacenada y organizada en las tablas de la base de datos que se va a desarrollar con el gestor MySql. 
Una vez aclarados los puntos de partida, procedemos a delimitar la información necesaria con el fin de generar criterios de selección sobre la información que debemos procesar.

En el Diagrama 3 observamos un esquema de los métodos que se utilizaron para procesar la información. Se parte de que después de recorrer el fichero y de analizar su contenido se verifique si existen parámetros invariables, y si existiera crearíamos una tabla llamada Cabecera de archivo (Cab_Arc), y de existir parámetros variables aplicaríamos una tabla cuyo nombre sea detalle o descripción de las detecciones (Dis_det). Hecho esto analizamos la información (figura 43) y al mismo tiempo se irán predefiniendo los campos para esquematizar la base de datos en su primera instancia.

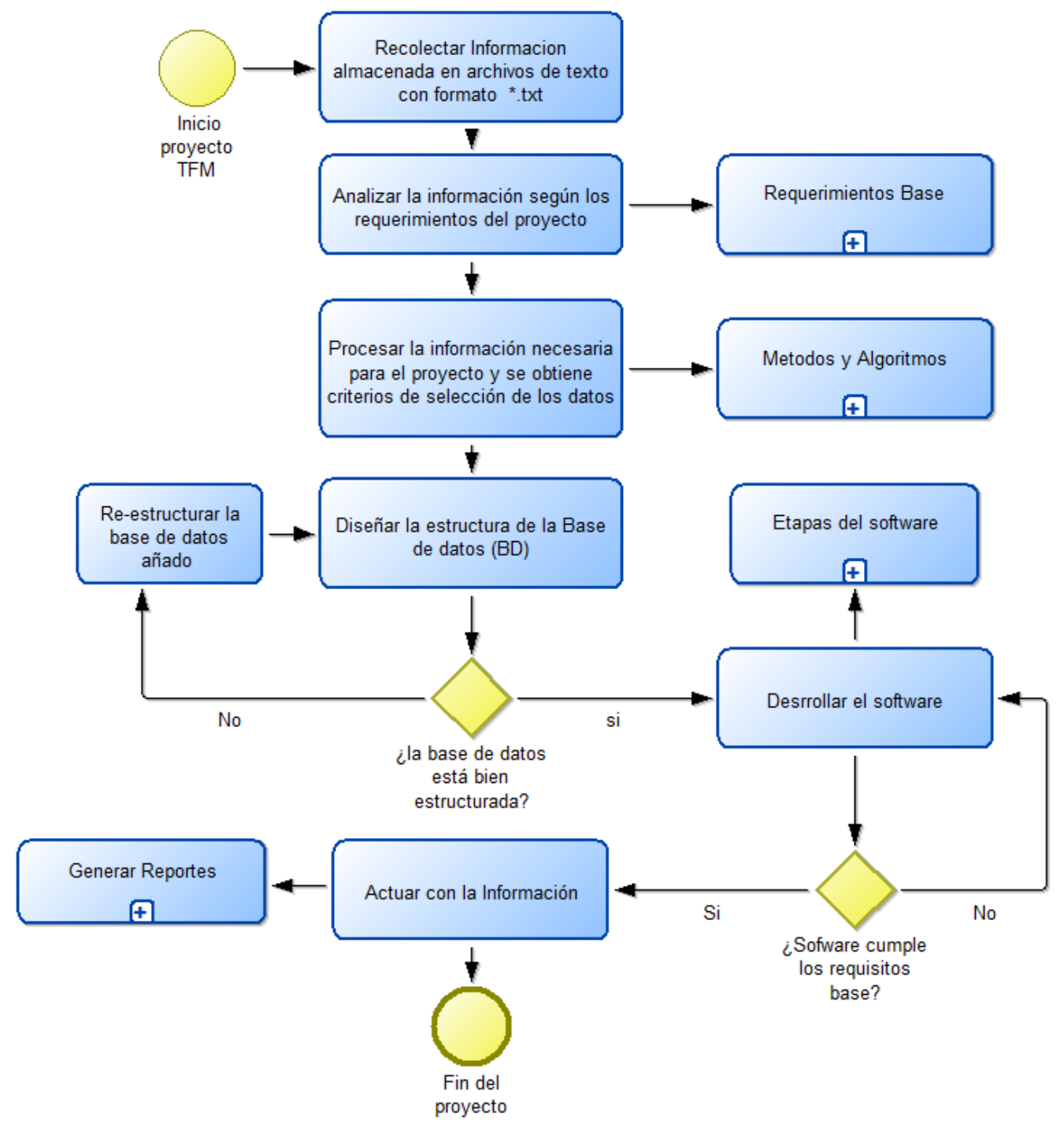

Diagrama 1. Diagrama general de la arquitectura de desarrollo 


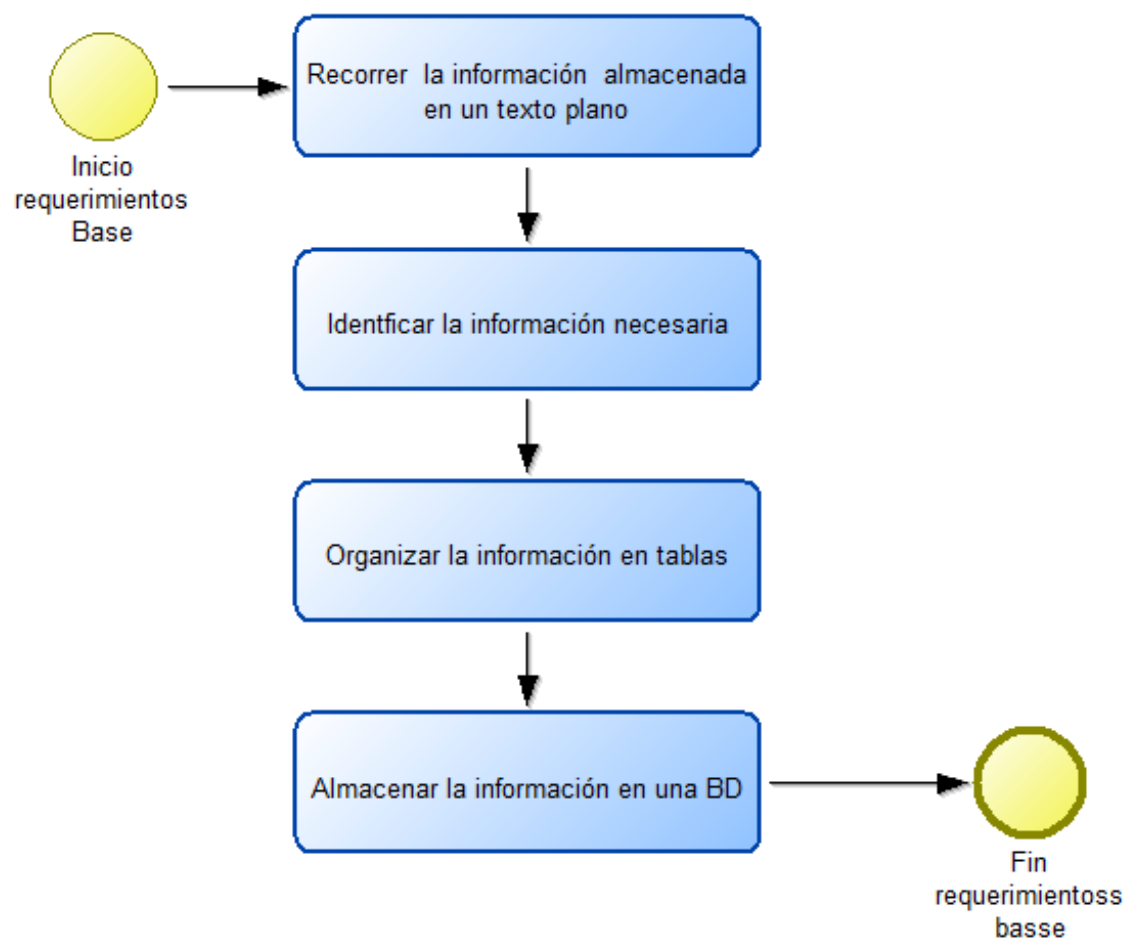

Diagrama 2. Diagrama requisitos base del sistema 


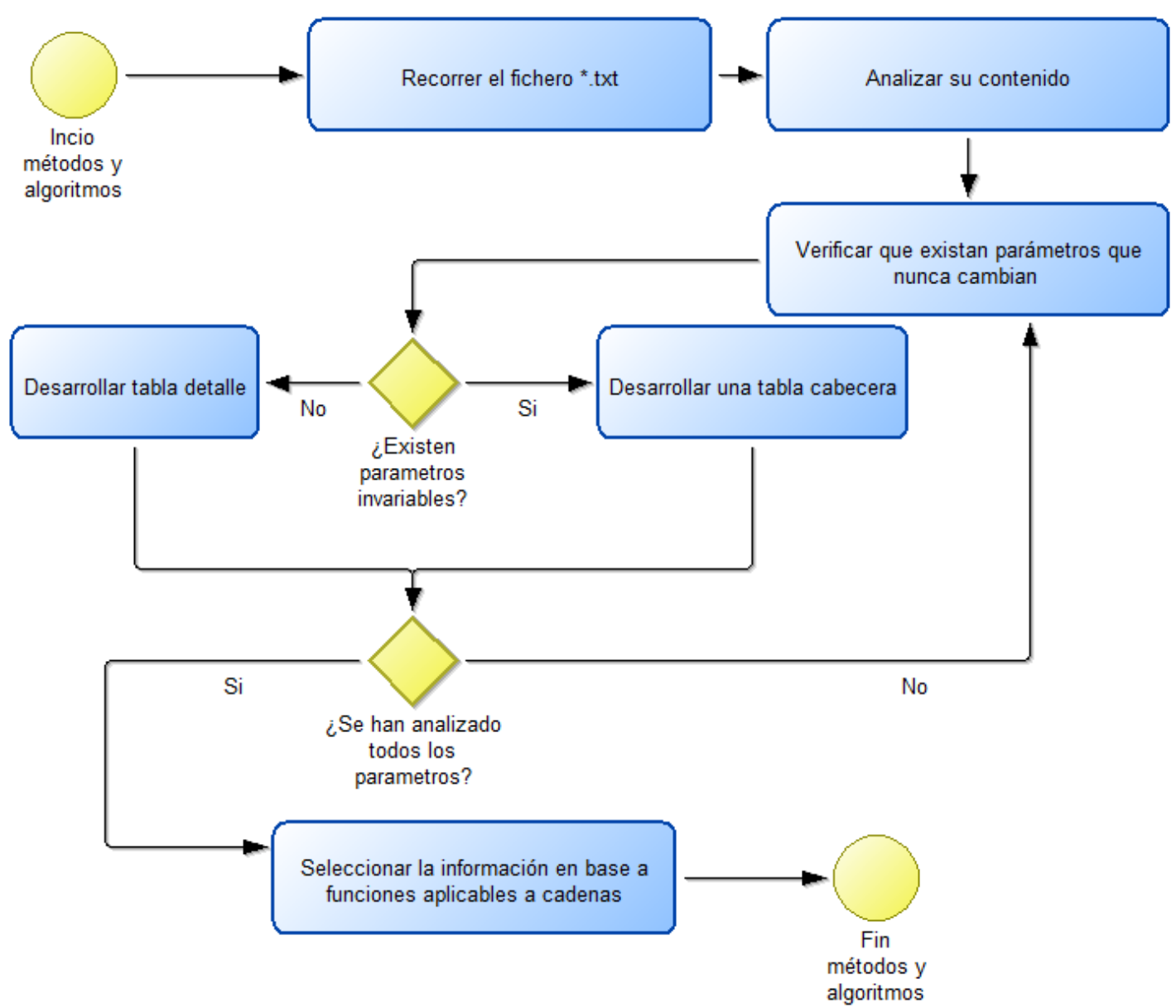

Diagrama 3. Diagrama requisitos base del sistema

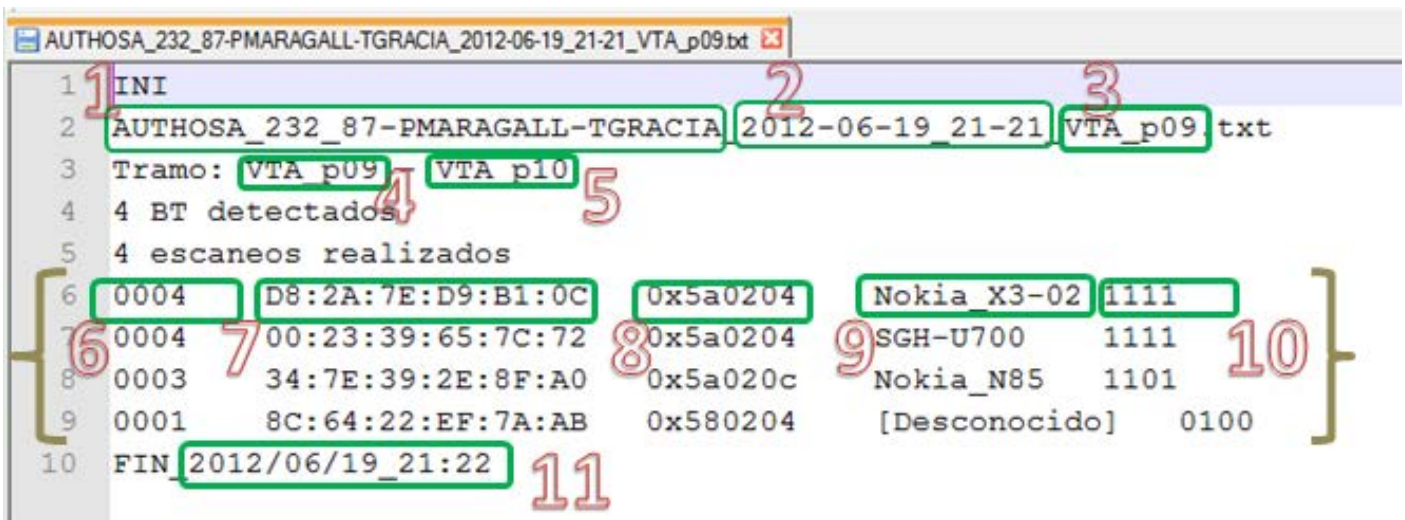

Figura 43. Archivo de texto plano generado por el dispositivo Bluetooth: (1) Línea de autobús; (2) fecha y hora de inicio de recorrido; (3..4) parada de origen; (5) parada destino; (6) número de veces que ha sido detectado por el dispositivo bluetooth; (7) MAC del dispositivo; (8) código del fabricante; (9) nombre del dispositivo; (10) vector de detecciones; (11) fecha y hora de acabado del recorrido.

Partiendo de un pre-diseño de la base de datos (Las tablas Cabecera y Detalle relacionados por un campo clave) se definen las tablas adicionales necesarias, ver figura 44. 


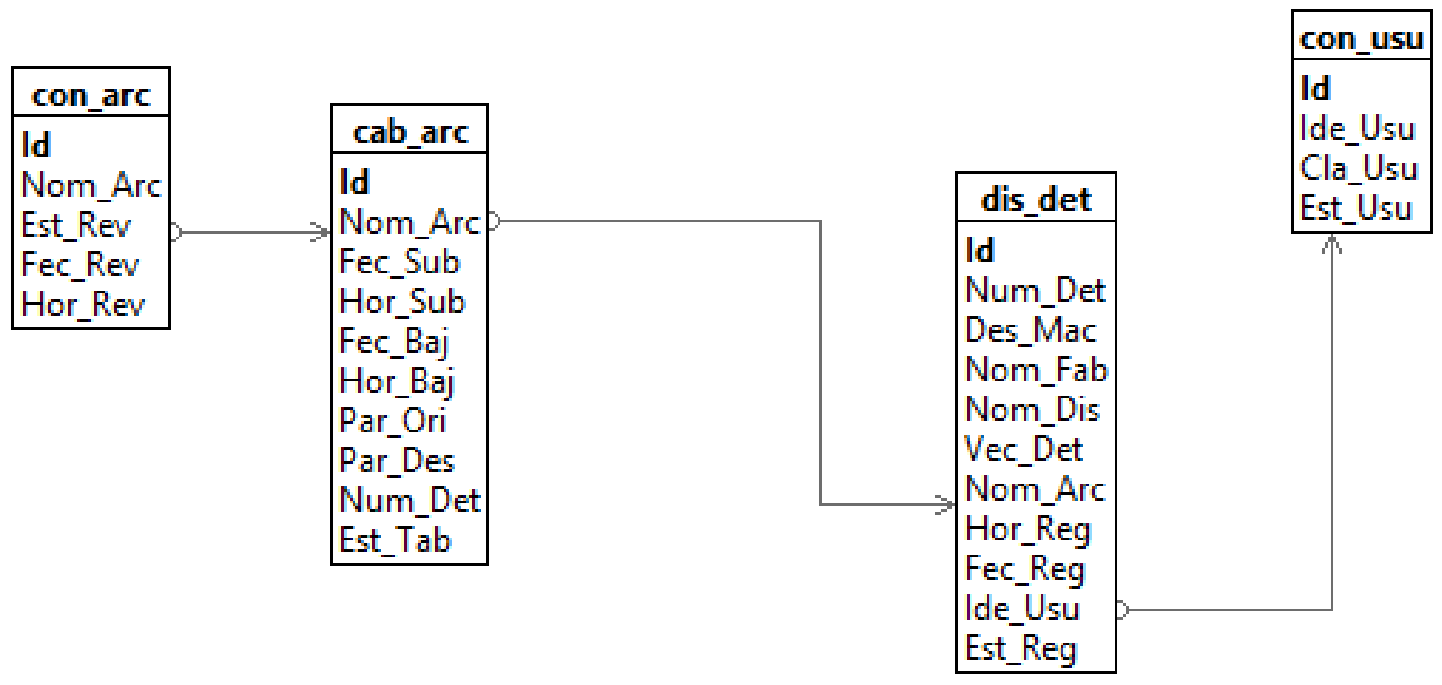

Figura 44. Diseño de la base de datos CtrlBus (control de autobús) con las siguientes tablas: control de archivos (Con_arc); cabecera de archivo (cab_arc); dispositivos detectados (dis_det) y una tabla para control de usuarios (con_usu).

Continuando con la secuencia del diagrama General Diagrama 1 se analizará la estructura de la base de datos para comprobar si cumple con los requisitos (comprobar que la BD contenga la información requerida que se muestra en la figura 43), también podemos ver la Tabla 2 y Tabla 3 que nos ejemplifica una pequeña comprobación de la información que necesitamos comparándola con la información necesaria expresada en la figura anteriormente mencionada.

\begin{tabular}{|c|c|c|}
\hline \multirow{4}{*}{ Dis_Det } & Campo & Descripción \\
\cline { 2 - 3 } & Num_Det & Número de detecciones ver Figura 43 (6) \\
\cline { 2 - 3 } & Des_Mac & Descripción de la Mac ver Figura 43 (7) \\
\cline { 2 - 3 } & Nom_Fab & Nombre del Fabricante ver Figura 43 (8) \\
\cline { 2 - 3 } & Nom_Dis & Nombre del dispositivo ver Figura 43 (9) \\
\cline { 2 - 3 } & Vec_Det & Vector de detecciones ver Figura 43 (10) \\
\cline { 2 - 3 } & Nom_Arc & Nombre del archivo ver Figura 43. Segunda línea del \\
& fichero \\
\hline
\end{tabular}

Tabla 2. Comprobación de los requisitos tabla dispositivos detectados

\begin{tabular}{|c|c|c|}
\hline \multirow{4}{*}{ Cab_Arc } & Campo & Descripción \\
\cline { 2 - 3 } & Nom_Arc & $\begin{array}{c}\text { Nombre del archivo ver Figura 43. Segunda línea del } \\
\text { fichero. }\end{array}$ \\
\cline { 2 - 3 } & Fec_Sub & Fecha de subida ver Figura 43 (2). \\
\cline { 2 - 3 } & Hor_Sub & Hora de subida ver Figura 43 (2). \\
\cline { 2 - 3 } & Fec_Baj & Fecha de bajada ver Figura 43 (11). \\
\cline { 2 - 3 } & Hor_Baj & Hora de bajada ver Figura 43 (11). \\
\hline
\end{tabular}




\begin{tabular}{|c|c|c|}
\hline \multirow{2}{*}{} & Par_Or & Parada origen ver Figura 43 (4). \\
\cline { 2 - 3 } & Par_Des & Parada destino ver Figura 43 (5). \\
\cline { 2 - 3 } & Num_Det & $\begin{array}{c}\text { Numero de detecciones ver cuarta línea del fichero de } \\
\text { texto Figura 43 }\end{array}$ \\
\hline
\end{tabular}

Tabla 3. Comprobación de los requisitos tabla cabecera de archivo

Una vez comprobados los datos necesarios para cumplir con los requisitos del sistema, se elabora el software para generar los informes (matriz origendestino). Para el desarrollo del software se realizó el siguiente diagrama ver Diagrama 4, en el que se explican los procesos a seguir para el desarrollo del mismo.

Primero se establecen los criterios de organización de la información, para luego programarlo y verificar que se adecúen a lo que realmente necesitamos. Para ello, se almacena la información temporalmente en matrices (vectores bidimensionales), se prueban y se verifican los resultados por pantalla. Por último, se establecen las sentencias SQL que serán utilizadas.

Las sentencias serán testeadas en primera instancia en el editor SQL del software de MySql-front. Esto ayudará a realizar unas sentencias más robustas y concretas. Una vez completado este proceso, la información ya será apta para su registro en la base de datos. 


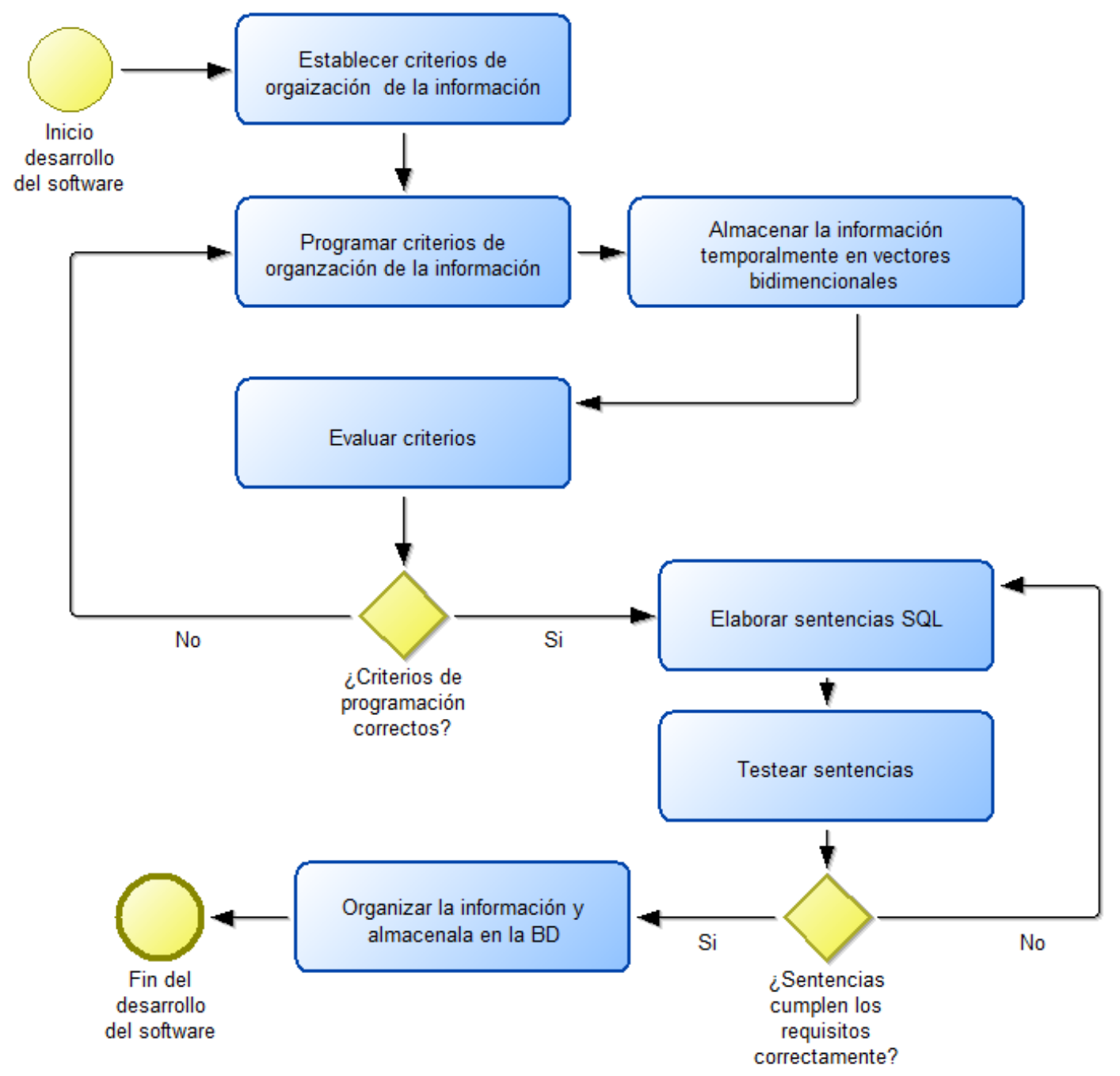

Diagrama 4. Procesos considerados para este desarrollo software

Como se puede apreciar en el Diagrama 5, para la creación de reportes se establecen filtros que permitirán mostrar u ocultar la información en base un campo que se ha establecido en la tabla dis_det y cab_arc (ver Figura 44), donde la tabla dis_det posee un campo Est_Tab que permite dar de baja o activar el archivo de texto, y la tabla dis_det dispone de un campo llamado Est_Reg que permitirá determinar si la línea de detección es correcta o no.

En ningún momento se elimina la información de la base de datos puesto que se trabaja con estados (atributo estado) que permitirá activar o desactivar el registro. 


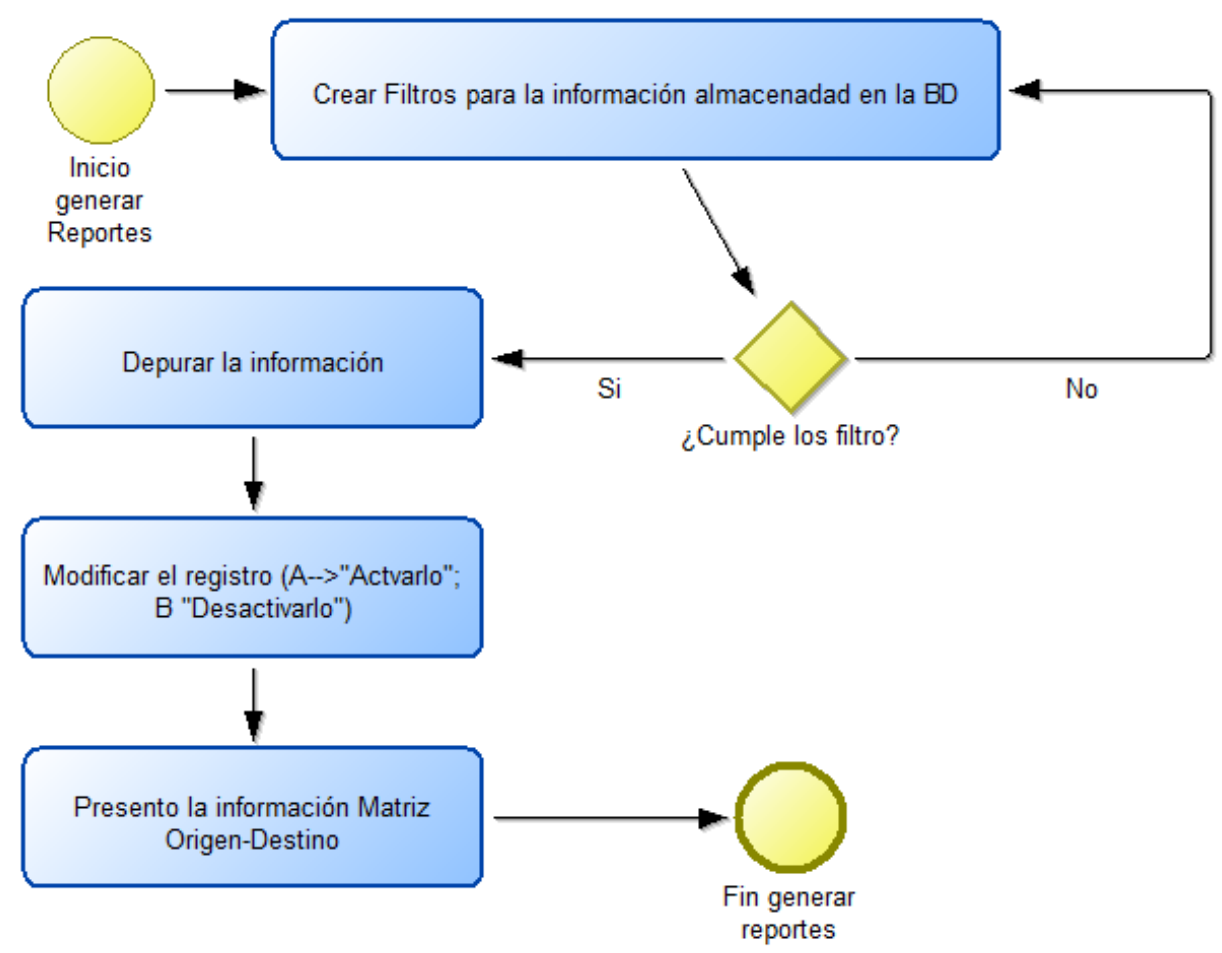

Diagrama 5. Procesos para generar reportes

\subsubsection{Análisis y diseño de la base de datos.}

Para el diseño de la base de datos se ha establecido una nomenclatura y estructura de caracteres. Los nombres de las tablas y de los campos deben hacer referencia a la información que almacena cada tabla y cada campo. Otra norma que se aplica para establecer el nombre, tanto de la tabla como el campo, deberá ser la siguiente: se optará por las tres primeras letras de la equivalencia a la palabra del campo y la separación se realizará por medio del signo underscore "_" o guion bajo. Es decir, para definir el campo nombre de archivo seleccionaremos las 3 iniciales de cada palabra descriptiva como se mencionó anteriormente y como resultado obtendríamos el siguiente campo Nom_Arc $\rightarrow$ "Nombre de archivo" ver figura 44 para contemplar cada uno de los nombres de los campos de la base de datos.

El diseño de la base de datos parte de una tabla cabecera de archivo y otra de detalle o dispositivos detectados, es decir, tendríamos una relación de uno a muchos, donde una cabecera puede tener de uno a varios dispositivos detectados y un dispositivo detectado tendría una sola cabecera en ese trayecto.

Para controlar quien accede a esta información se ha considerado una tabla usuarios (Tabla 4) que nos permitirá trabajar con sesiones de usuarios. De la misma manera, se ha creado una tabla de control (Tabla 5) que es la que nos permitirá 
controlar los archivos ya procesados, el instante de tiempo en que se procesaron y qué usuario intervino en la realización de dichas operaciones.

\begin{tabular}{|c|c|c|}
\hline \multirow{4}{*}{ Con_Usu } & Campo & Descripción \\
\cline { 2 - 3 } & Ide_Usu & Identificación de usuario \\
\cline { 2 - 3 } & Cla_Usu & Clave de usuario \\
\cline { 2 - 3 } & Est_Usu & Estado de Usuario "A" $\rightarrow$ Alta; "B" $\rightarrow$ Baja \\
\hline
\end{tabular}

Tabla 4. Tabla control de usuario

\begin{tabular}{|c|c|c|}
\hline \multirow{4}{*}{ Con_Arc } & Campo & Descripción \\
\cline { 2 - 3 } & Nom_Arc & Nombre del archivo \\
\cline { 2 - 3 } & Fec_Rev & Fecha de revisión del archivo. \\
\cline { 2 - 3 } & Hor_Rev & Hora de revisión del archivo. \\
\cline { 2 - 3 } & Est_Rev & Estado de archivo "A" $\rightarrow$ Alta; "B" $\rightarrow$ Baja \\
\hline
\end{tabular}

Tabla 5. Tabla control archivo

\subsubsection{Software de simulación}

A continuación se describe el software desarrollado en base a los objetivos planteados inicialmente. En los próximos apartados se hablará de las funciones claves del sistema y de las principales líneas de programación.

Se presentará un diagrama que englobe las funciones del sistema. Además, se justificara el uso de alguna instrucción que sea considerada necesaria para esta aplicación.

\subsubsection{Diseño funcional}

Para este desarrollo se ha elegido el lenguaje de programación Php, por ser un lenguaje muy potente y ampliamente utilizado en entornos web. Los datos provienen del sistema de detección descrito en anteriores apartados en forma de texto plano. Este dispositivo se encuentra embarcado en el autobús y comienza a realizar detecciones periódicas de dispositivos bluetooth. Cuando el autobús parte de una parada origen hacia una parada destino ver figura 45. 


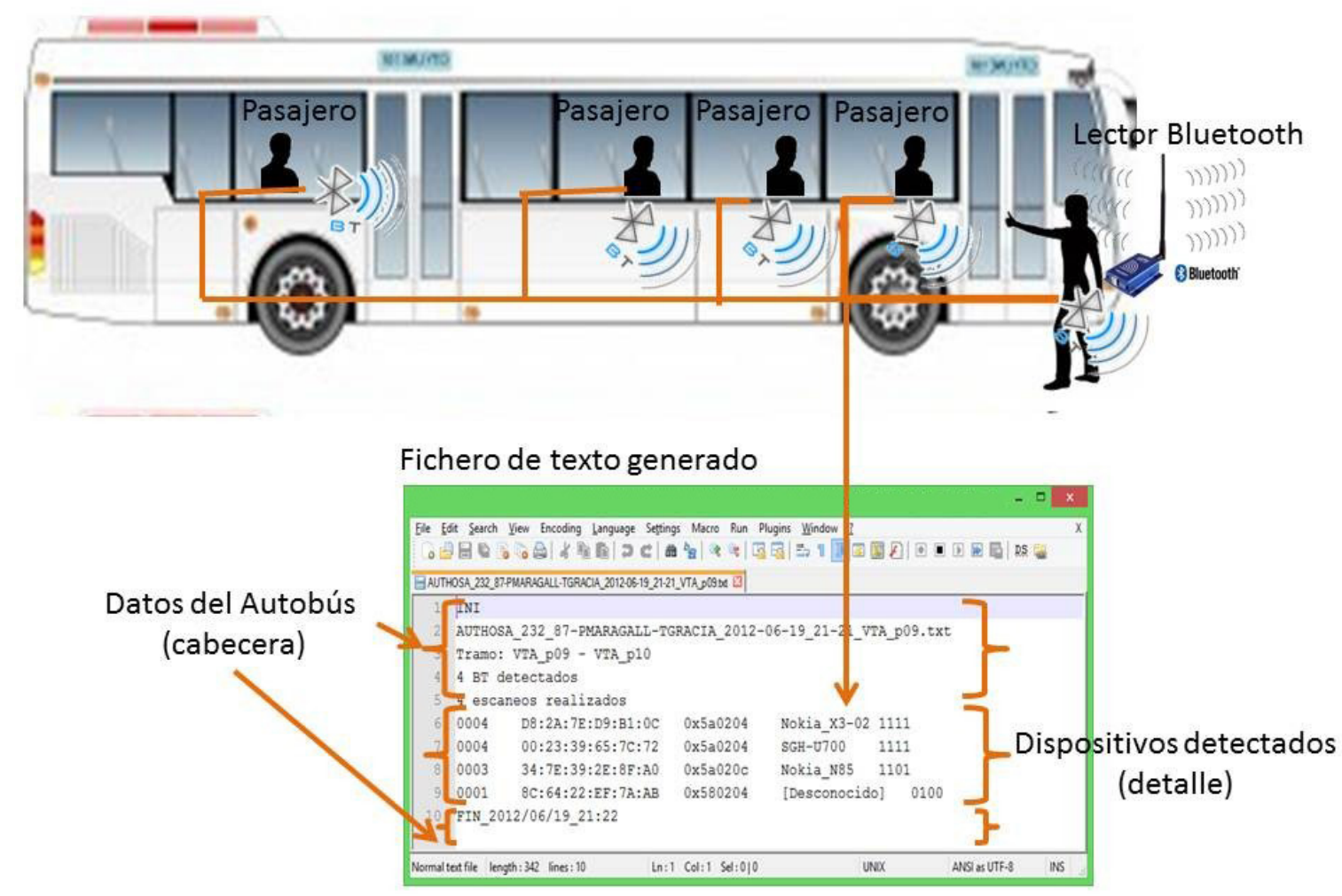

Figura 45. Esquema del funcionamiento del software externo que me permitirá recolectar la información de los pasajeros.

El archivo de texto plano que genera el sistema de detección lo podemos observar en la figura 45, la información de estas detecciones será almacenada con un formato único para todos los archivos.

Haciendo una breve reseña de los procesos del sistema desde su ejecución hasta el envío de la información a la base de datos y su correspondiente tratamiento, se seguirá la siguiente secuencia:

- Lanzamiento del sistema (vía web).

- Controlar de Usuario (Sesiones web).

- Subir archivos obtenidos previamente por la aplicación (aplicación externa).

- Procesar la información.

- Almacenar temporalmente en vectores.

- Realizar operaciones SQL (inserción, modificación, eliminación, etc.) en la base de datos.

- Generar matrices origen-destino. 
- Cálculos de probabilidad.

En el Diagrama 6 se describe la funcionalidad de esta parte del sistema.

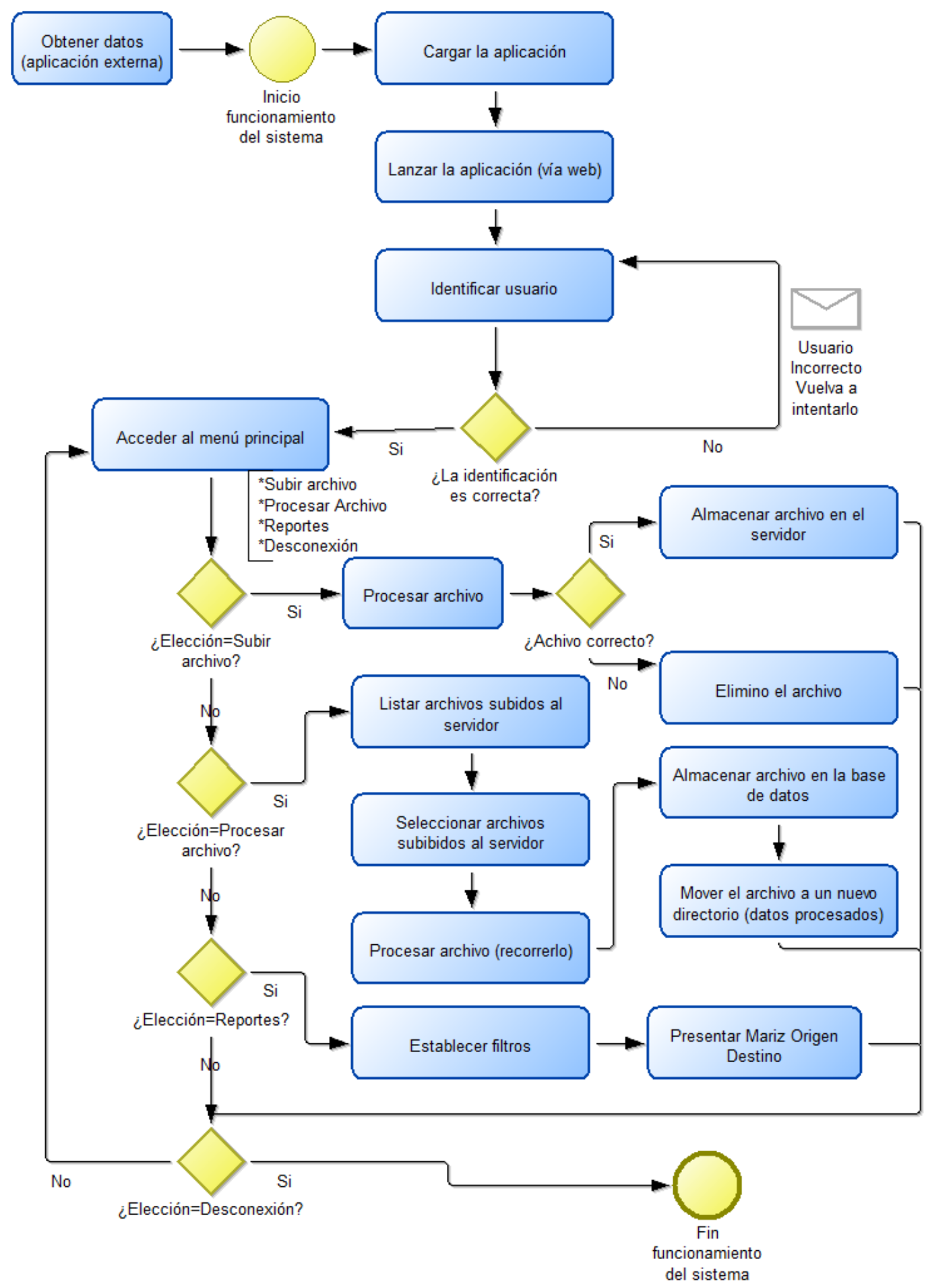

Diagrama 6. Procesos del sistema

\subsubsection{Desarrollo del software}

Para conseguir un desarrollo óptimo de la aplicación se ha optado por la programación en niveles o capas. La programación por capas es una arquitectura 
cliente-servidor en el que el objetivo primordial es la separación de la lógica de negocios de la lógica de diseño. Un ejemplo básico consiste en separar la capa de datos de la capa de presentación al usuario.

La ventaja principal de este estilo es que el desarrollo se puede llevar a cabo en varios niveles y, en caso de que sobrevenga algún cambio, sólo se ataca al nivel requerido sin tener que revisar una mayor parte del código. Además, permite la creación de un diseño escalable.

\subsubsection{Control de Usuario (Sesiones web).}

La base de datos que se utiliza contendrá una tabla para los usuarios llamada Con_Usu, control de usuario (ver figura 44 y más detalles en la Tabla 5). Esta tabla nos permitirá controlar el acceso al sistema por sesiones.

Primeramente se creó el fichero index.php, el cual será el primer archivo que se invoque para nuestro inicio de sesión. Prácticamente es puro código html (ver figura 46).

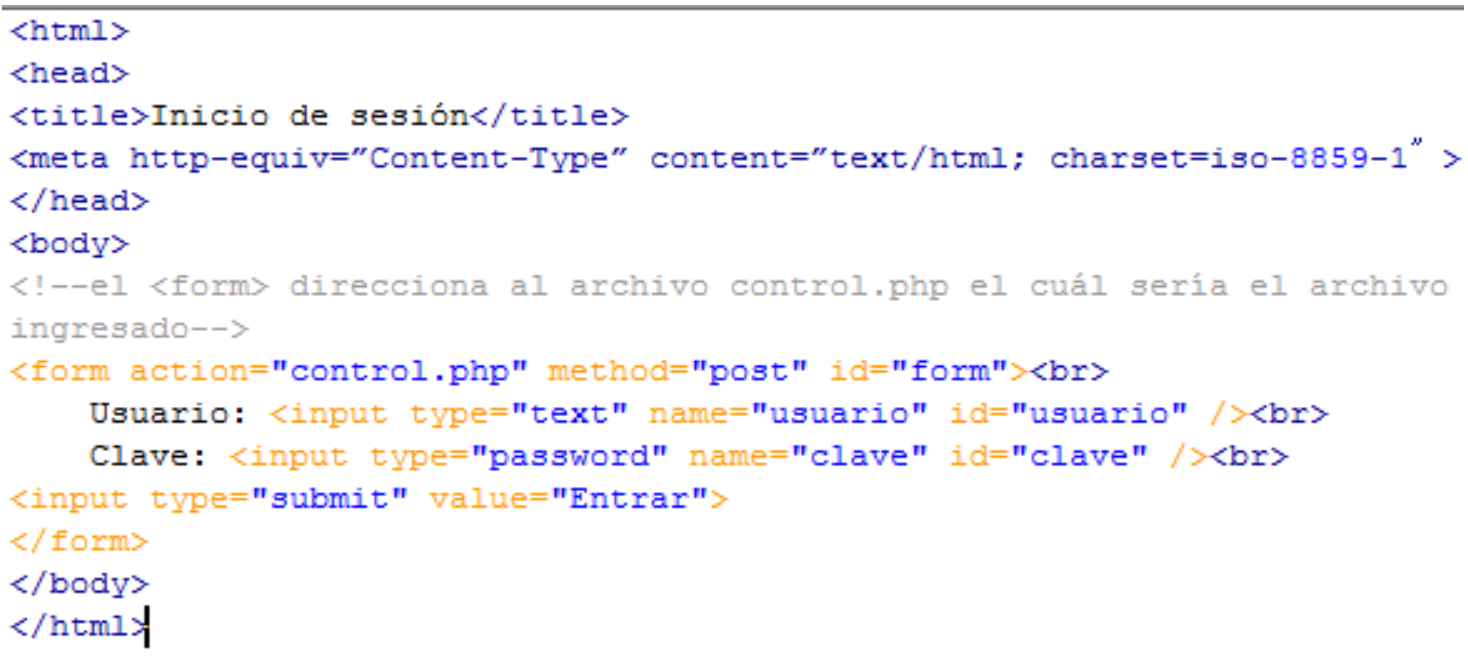

Figura 46. Página principal Index.php

Como se puede apreciar en la figura 46 , el $<$ form $>$ direcciona al archivo control.php el cuál sería el archivo encargado de realizar la validación del usuario y contraseña ingresado. En este archivo establecemos la conexión a la base de datos (figura 48) y verificamos el usuario tal como se muestra en la figura 47. 


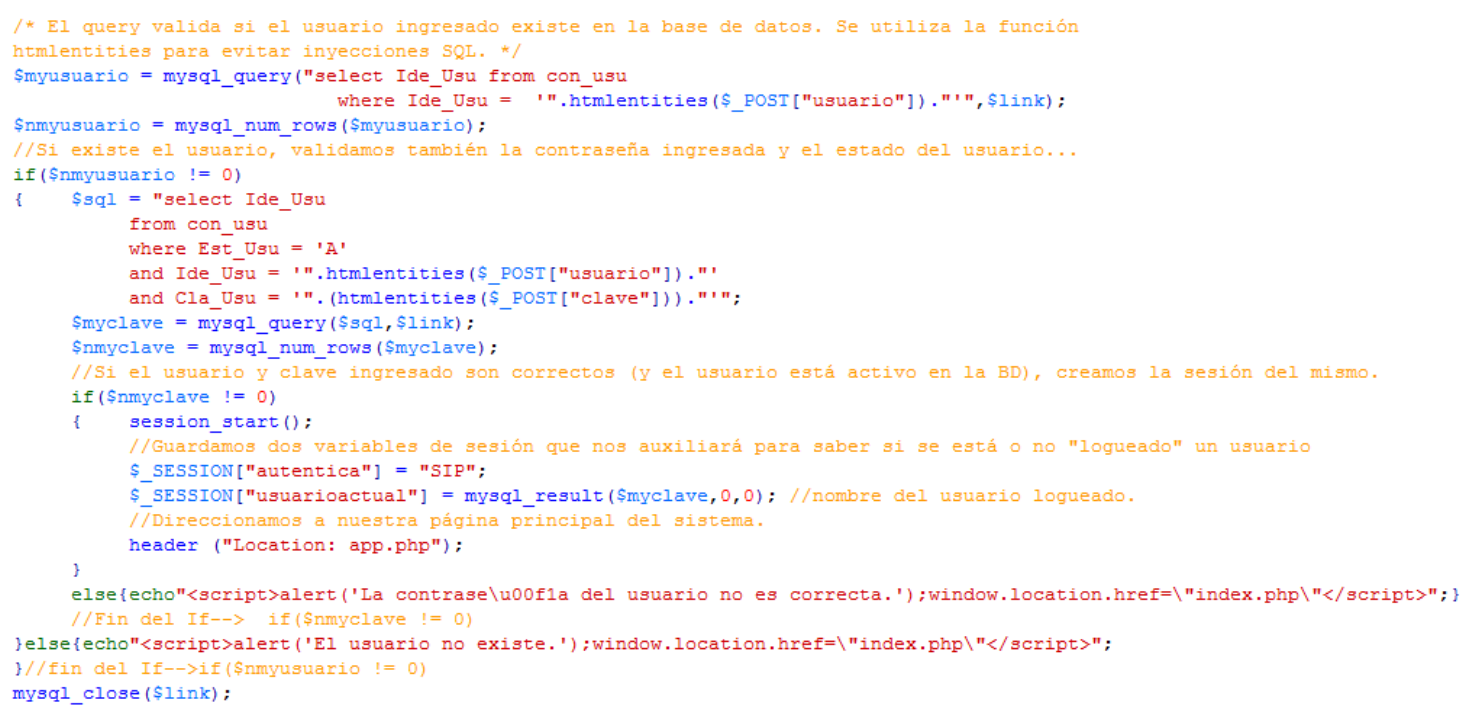

Figura 47. Archivo Control.php

Una vez verificado si el usuario existe el sistema le dará acceso al menú de opciones del sistema, que le permitirá procesar la información de acuerdo a las opciones de seguridad que se ha desarrollado y podrá acceder al menú de opciones.

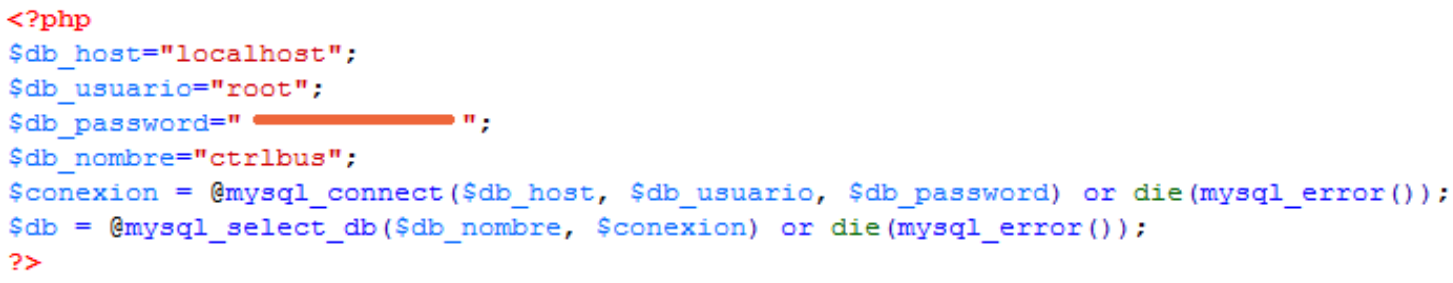

Figura 48. Código de conexión a la BD

Si el login es correcto llamaremos a la página inicial en la cual comprobaremos que existe realmente una sesión activa porque de lo contrario regresaríamos al usuario a la pantalla de comprobación de usuarios para volver a validar sus datos ver figura 49.

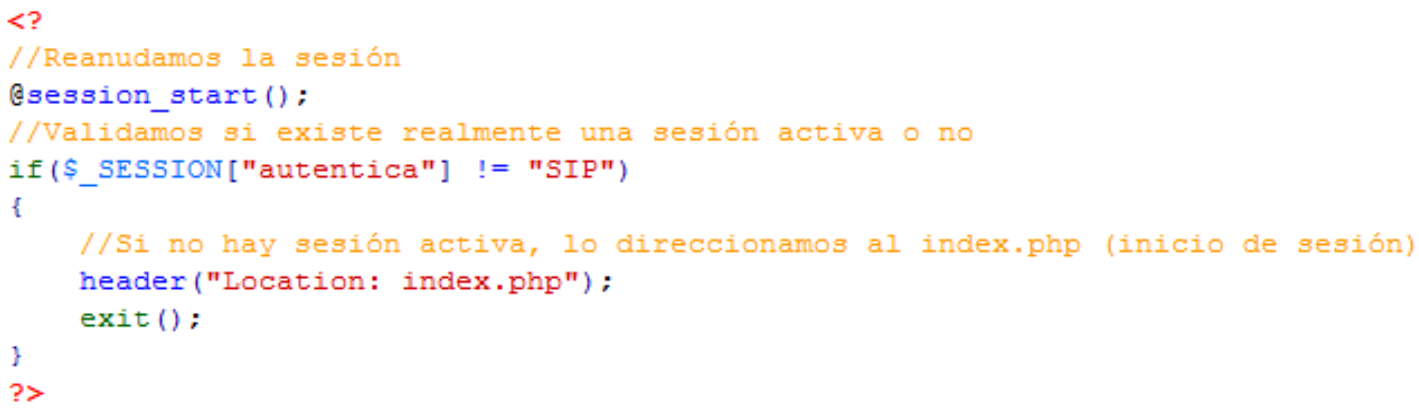

Figura 49. Control de sesiones activas.

La interfaz de usuario inicial se muestra en la figura 50 , donde se pueden apreciar los campos requeridos para el control de usuario. 


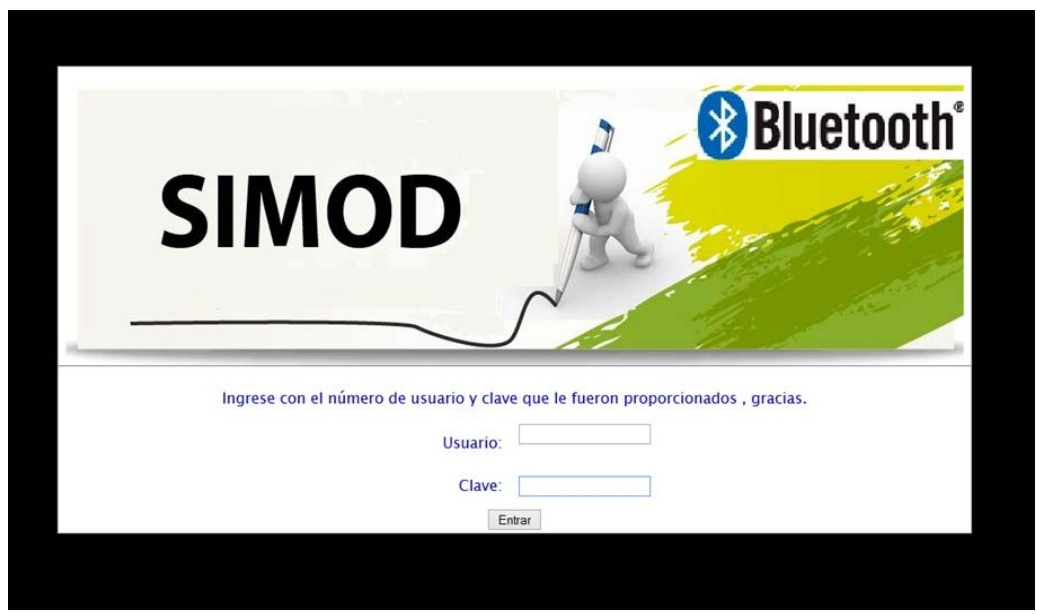

Figura 50. Control de usuarios en la capa de presentación

\subsection{Subir archivos (*.Txt)}

Para analizar el tratamiento de la información (los archivos tipo texto generados por el sistema de detección) debemos tener obligatoriamente los archivos en nuestro servidor. Para ello, después de haber activado una sesión de usuario tendremos disponible la opción de menú en la capa de presentación. El menú principal en formato web se presenta en la figura 51.

Una vez que tenemos acceso al menú principal debemos optar por la primera opción disponible (cargar archivos al servidor).

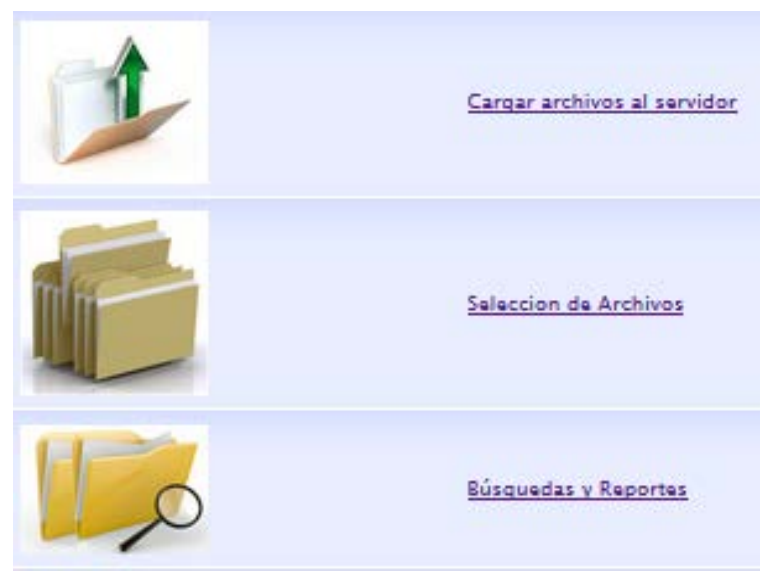

Figura 51. Menú principal

El siguiente paso es seleccionar el archivo y enviarlo al servidor como se muestra gráficamente en la figura 52 . 

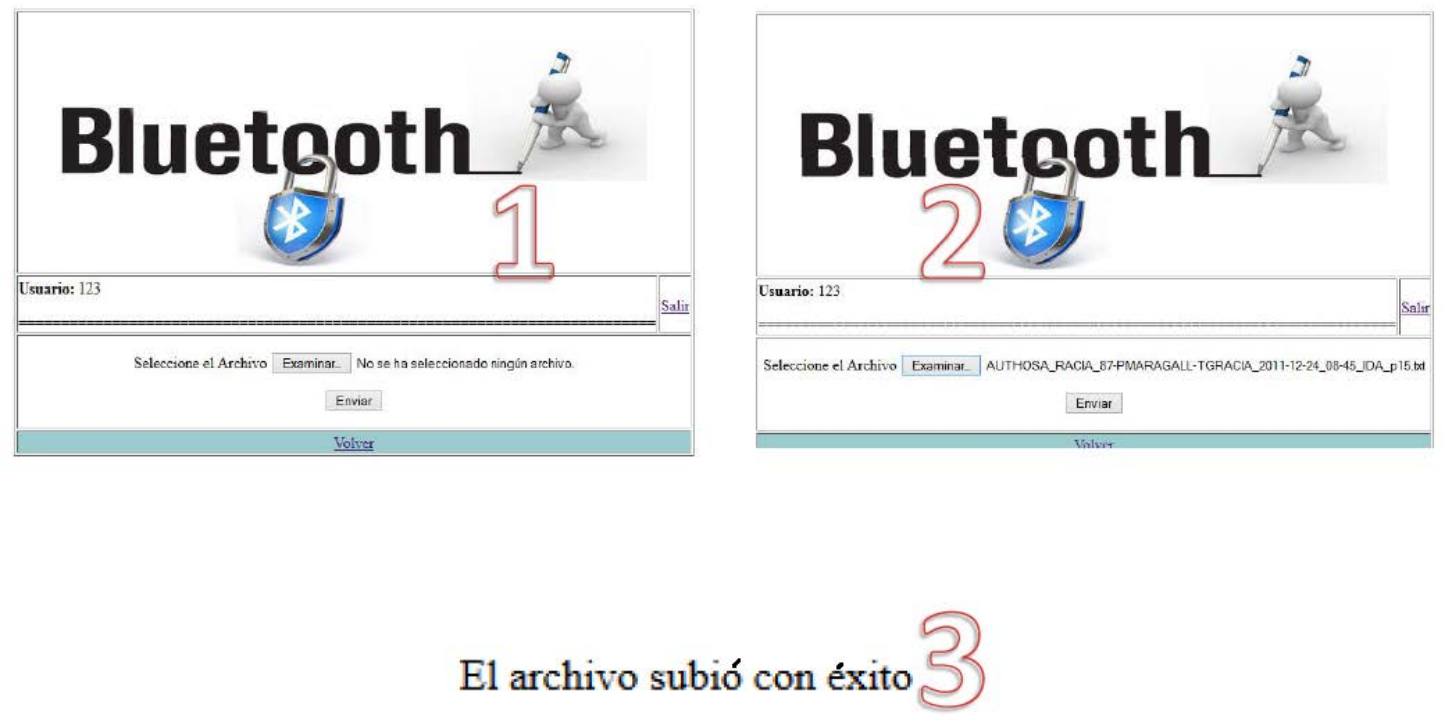

Figura 52. Pasos para cargar archivos al servidor (1) selecciono el archivo; (2) archivo seleccionado; (3) mensaje de confirmación.

Una vez cargado el archivo, quedara listo para ser procesado en el directorio raíz del servidor. Para que el archivo sea válido, debe ser texto plano (*.txt) y que contenga los parámetros predefinidos por el sistema como la palabra inicio en la primera línea y fin en la última. Así mismo, el archivo debe contener un mínimo de 6 líneas que serían las líneas que dan forma a la tabla cabecera del proyecto.

\subsection{Procesado de la información}

Una vez seleccionados los ficheros disponibles del listado de archivos (ver figura 53), se cargan en la base de datos.

\subsection{Almacenamiento y selección de la información}

Para seleccionar la información que se necesita almacenar, el primer paso es recorrer el archivo. Una vez hecho esto, se almacenará la información en vectores tipo matriz que permitirán organizar dicha información para seguidamente enviarla a la base de datos MySql. Este proceso se describe en el Diagrama 7.

En la figura 53 se muestra la selección de archivos en el paso 1 y el envío de archivos en el paso 2. Si la carga es exitosa, se visualizará un resumen como el mostrado en la figura 54. 


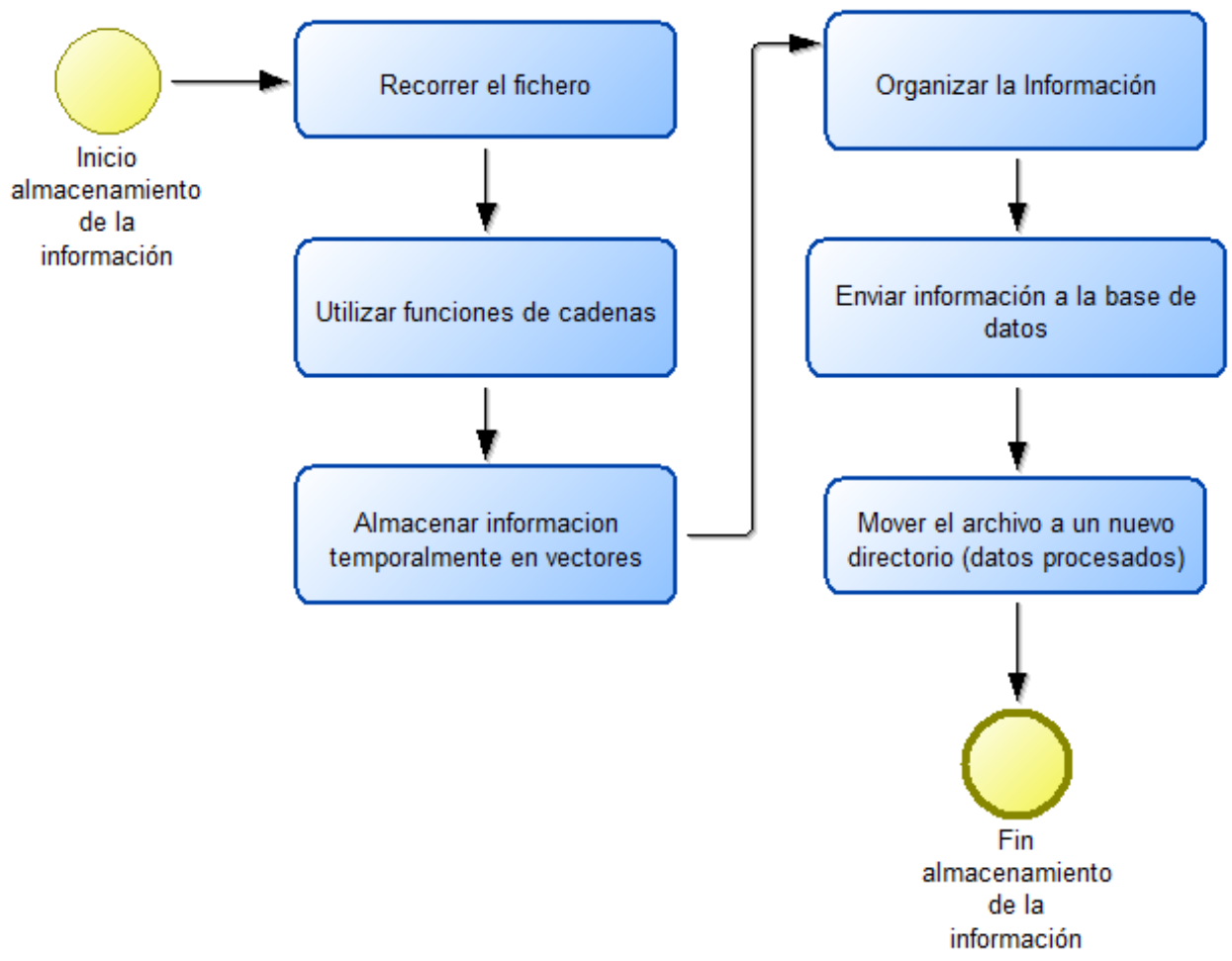

Diagrama 7. Procesos considerados para el desarrollo del software 

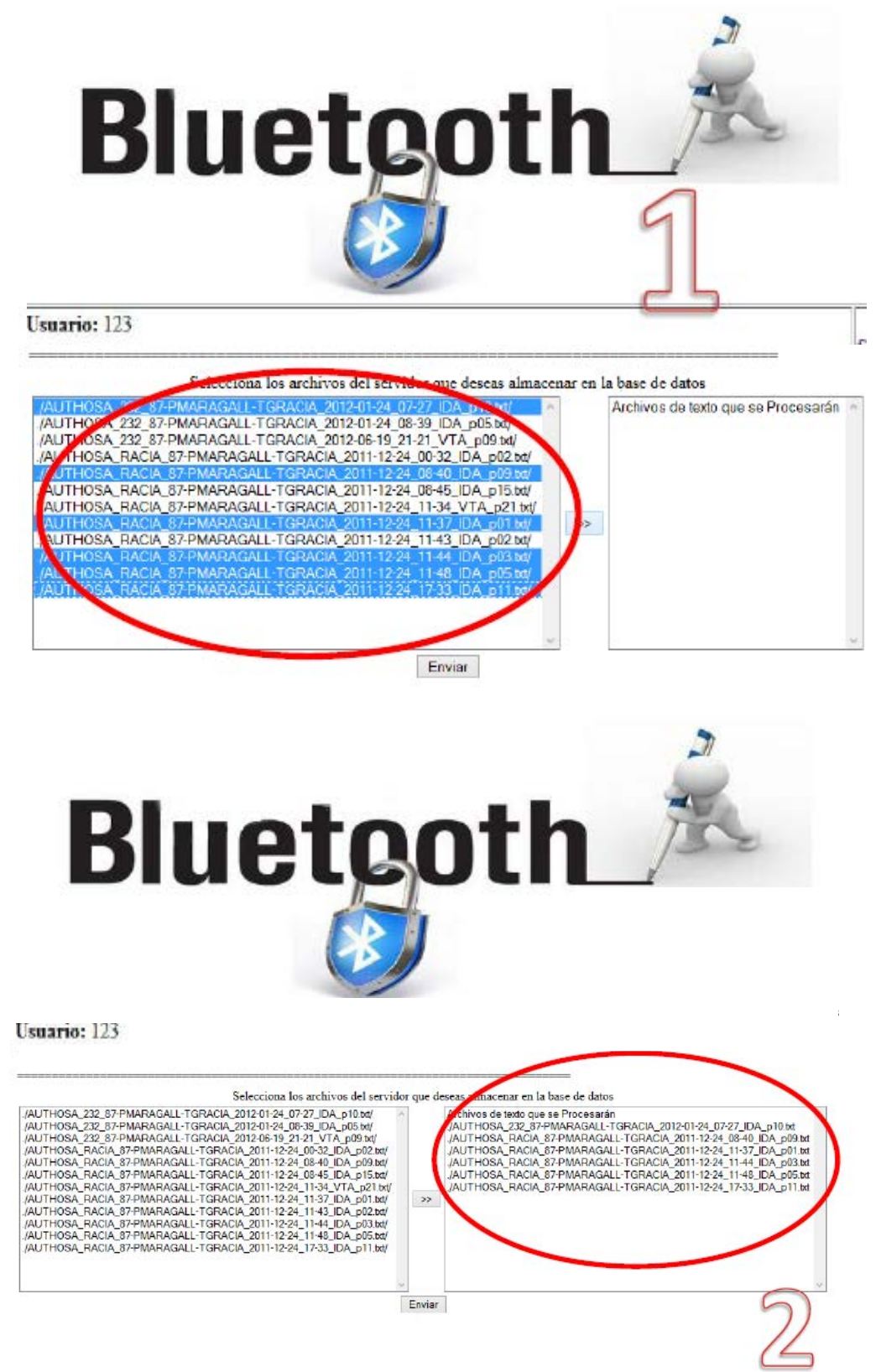

Figura 53. Pantalla del menú selección de archivos (1) Selección de archivos; (2) Archivos listos para procesar. 


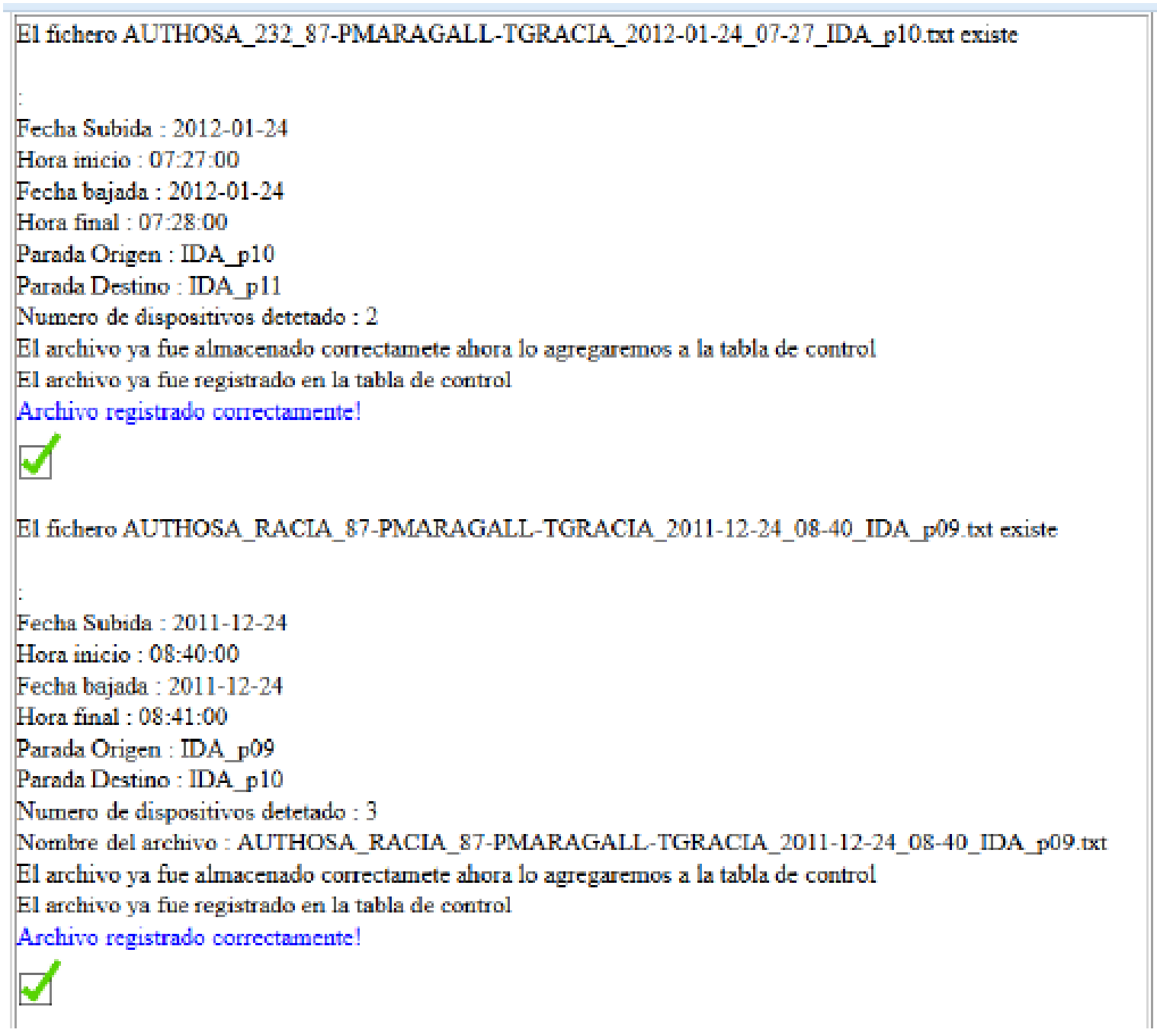

Figura 54. Mensaje de almacenamiento correcto en la base de datos.

A continuación se presentan algunas de las funciones aplicadas para la extracción de la información (tabla 6).

\begin{tabular}{|c|c|c|}
\hline & Función & Descripción \\
\hline \multirow{4}{*}{ Cadenas } & strlen & Obtiene la longitud de un string. \\
\hline & substr & Devuelve parte de una cadena. \\
\hline & substr_replace & $\begin{array}{l}\text { Reemplaza el texto dentro de una porción de un } \\
\text { string. }\end{array}$ \\
\hline & split & $\begin{array}{l}\text { Divide una cadena en una matriz mediante una } \\
\text { expresión regular. }\end{array}$ \\
\hline \multirow{6}{*}{ Archivos } & fopen & Abre un fichero o una URL. \\
\hline & fwrite & Escritura de un archivo en modo binario seguro. \\
\hline & fclose & - Cierra un puntero a un archivo abierto \\
\hline & file_exists & Comprueba si existe un fichero o directorio. \\
\hline & File & Transfiere un fichero completo a un array. \\
\hline & Unlink & Borra un archivo. \\
\hline
\end{tabular}




\begin{tabular}{|c|c|c|}
\hline & Mkdir & Crea un directorio \\
\cline { 2 - 3 } & move_uploaded_file & Mueve un archivo subido a una nueva ubicación \\
\hline
\end{tabular}

Tabla 6. Funciones para cadenas y manejo de archivos

\subsection{Informes}

Los informes permitirán determinar la matriz origen destino de la línea de autobús especificada, basándose en filtros de selección para la presentación de resultados. Para la obtención de reportes no se ha generado ninguna tabla adicional, por lo tanto, la base de datos queda tal cual estuvo en una primera instancia (ver figura 43). Los informes se generan a partir de sentencias SQL que filtran los datos. Para ello también se realiza una preselección de la información, es decir, verificar que los registros de nuestra tabla dispositivos detectados, estén considerados como válidos y no válidos, o no utilizables según corresponda.

Para que un registro sea considerado como válido, debe cumplir al menos alguno de los requisitos del apartado Filtrado de información del menú principal (figura 55).

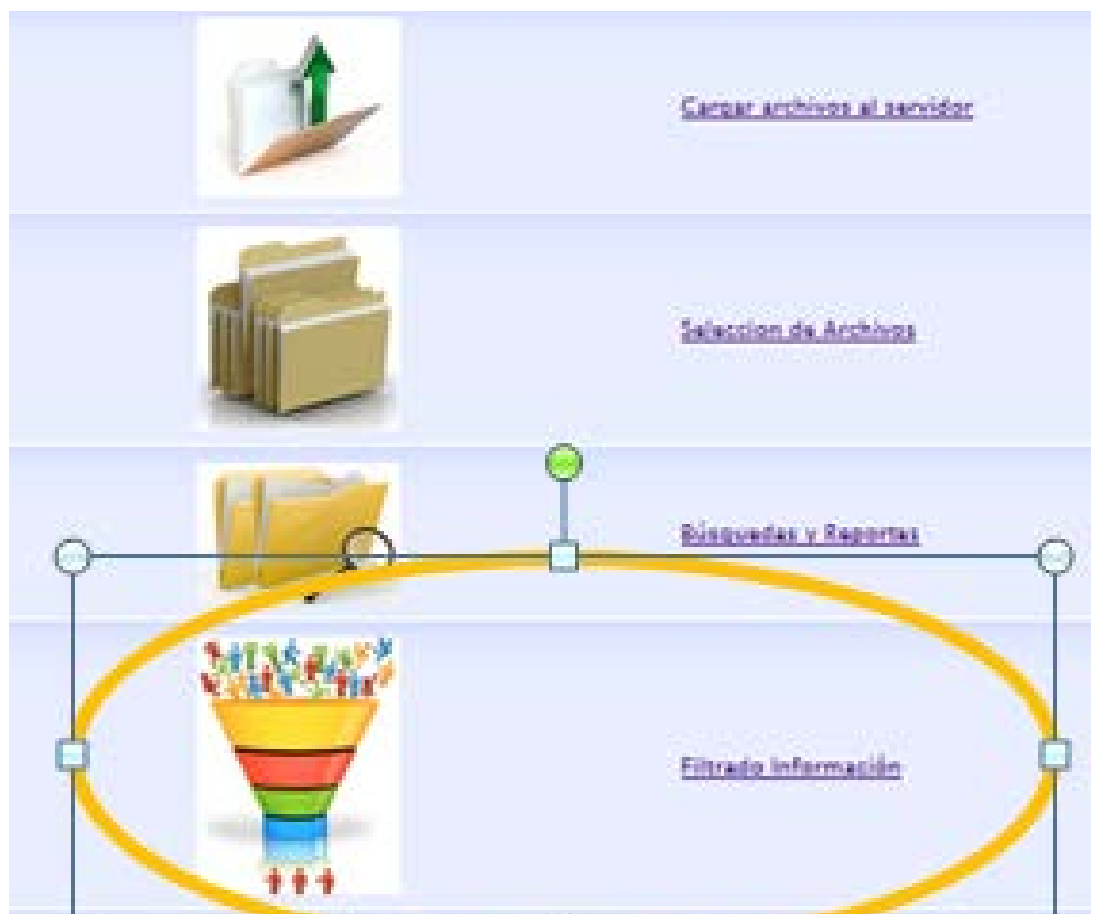

Figura 55. Opción: filtrado de datos del menú principal.

En este menú se pueden establecer criterios que deben cumplir los vectores de detección. En el registro serán dados de baja a través del campo estado todos aquellos que no cumplan los requisitos de selección y en futuras revisiones no será devuelto en las consultas SQL. 


\subsection{Matriz O-D}

Para la elaboración de la matriz O-D es necesario comprender cómo se lleva a cabo el proceso real de adquisición de datos. Para ello, se ha desarrollado un esquema en el que se detalla cómo es el proceso de selección de valores para la matriz O-D que presentaremos. Tomamos como referencia el número de paradas que ejecuta un autobús, es decir el recorrido que este realiza. En cada parada el autobús tiene la posibilidad de dejar pasajeros, recogerlos o no ejercer ninguna acción lo que excluiría dicha parada. Po ejemplo, en la figura 56 vemos que el autobús parte de la parada nำ hasta la parada no14 en ese trayecto ha habido paradas en las cuales se ha detenido a dejar pasajeros o a subirlos al autobús, y en algunos casos ninguna de las dos alternativas anteriores. Cabe recalcar que por cada parada se genera un archivo como el de la figura 42, el cual proporcionará la información de cada pasajero que se encuentra en el autobús después de que el dispositivo genere varios barridos y se registre a través de un vector de control en el fichero tipo texto.
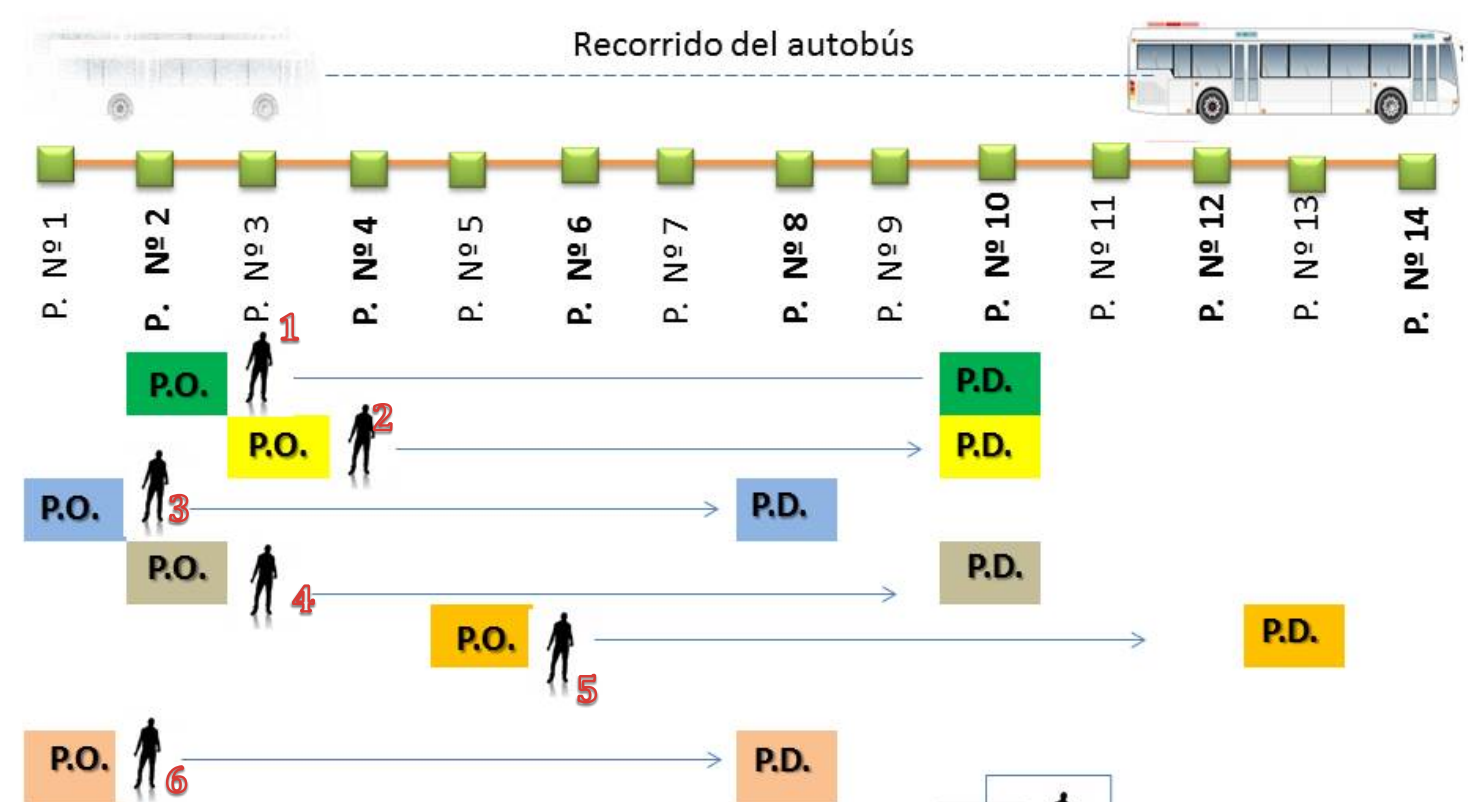

Leyenda

\begin{tabular}{|l|l|lll|}
\hline P2-P10 & P1-P8 & P5-P13 & P10 - P11 & Recorrido \\
P3-P10 & P2-P10 & P1-P8 & $\begin{array}{l}\text { P.O. Parada Origen } \\
\text { P.D. Parada Destino }\end{array}$
\end{tabular}

Figura 56. Proceso de determinación de parada origen y parada destino del pasajero. 
Continuando con el análisis de la figura 56 vemos que entre los trayectos de autobús (P. №1 hasta P. №14) han intervenido 7 pasajeros, que han subido y han bajado, algunos en paradas diferentes y otros en las mismas. Por ejemplo, el pasajero №1 sube en la parada №2 y se baja en la parada №10, teniendo el mismo recorrido que el pasajero № 4 lo cual indica que en la matriz de origen-destino habrán dos pasajeros que permanecieron en el mismo recorrido del autobús desde la parada inicial, en este caso la №2 y su destino final que fue la parada №10

Trataremos la matriz origen-destino que construimos en base a los criterios de selección mencionados anteriormente, agrupando registros y clasificándolos para que el procesado de información ofrezca un resultado coherente, se puede decir que la matriz origen-destino nos permitirá determinar cuántas personas han hecho uso del servicio de autobús, es decir cuántas personas han subido y cuántas han bajado de acuerdo al número de paradas, todo esto según su recorrido establecido (desde la parada №1 hasta la parada №14).

La matriz 0-D resultante de la figura 56 se muestra en la figura 57. Como se puede apreciar, la matriz nos indica que desde la parada №1 hasta la parada №8 de acuerdo con el recorrido del autobús han permanecido 2 personas en este trayecto, que se han subido en la parada №1 y se han bajado en la parada №8, al igual que en la parada №2 hasta la parada №10, mientras que desde la parada №3 hasta la №10 únicamente ha permanecido en el trayecto 1 persona al igual que de la parada №5 hasta la parada №13. Ahora, si nos fijamos en la figura 56 vemos que el pasajero №7 tiene parada de origen pero no para destino, con lo cual determinamos que se ha subido en la parada № 10 y se ha bajado en la parada más próxima en este caso la №11. De no ser así y si el pasajero tuviese aparición en los registros de forma esporádica se le consideraría como usuario valido tomando en cuenta que su dispositivo pudiese presentar fallos a la hora de la detección. Por ejemplo. Si el pasajero apareciese en el registro de la parada №1 y posteriormente apareciese en el registro de la parada № 6 y №7, se considera como pasajero válido y se diría que esta persona tuvo un recorrido desde la parada №1 hasta la №7. De lo contrario se diría que el pasajero subió en la parada №1 y bajo en la siguiente en este caso la №2. Se deduce como pasajero válido porque se asume que su dispositivo puede estar averiado y presentar un mal funcionamiento. 


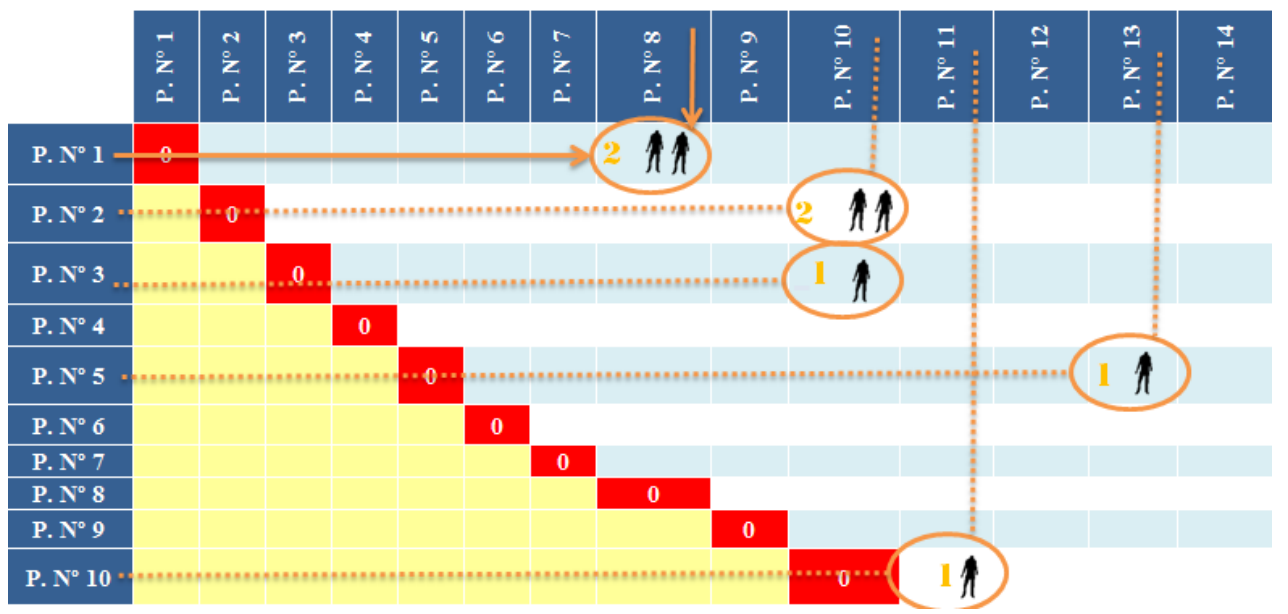

Figura 57. Matriz OD del trayecto de la parada №1 hasta la parada №14.

Ahora, en la matriz O-D se observa que su diagonal presenta como valor el número "0", lo que nos indica que en ese punto no es posible que se suba o baje alguien, porque estaríamos tratando con la misma parada y es un poco ilógico que alguien suba y baje en la misma parada. Es decir ninguna persona podría subir en la parada №1 y bajar en la parada №1 ni subir en la №2 y bajarse en la misma.

Una vez que hemos obtenido la matriz origen destino, estamos listos para obtener la matriz de probabilidad, que será explicada a continuación.

\subsection{Matriz de Probabilidad.}

La matriz de probabilidad se realiza basándose en la probabilidad de Laplace que nos dice que si un experimento cualquiera puede dar lugar a un número finito de resultados posibles, y no existe ninguna razón que privilegie unos resultados en contra de otros, se calcula la probabilidad de un suceso aleatorio A, según la regla de Laplace como el cociente entre el número de casos favorables a $\mathrm{A}$, y el de todos los posibles resultados del experimento (1) [Spiegel'1982].

$$
P_{[A]}=\frac{\text { Número de casos favorables a } A}{\text { Número de casos posibles }}
$$

Tomando en consideración la teoría de probabilidad de Laplace (1) podemos decir que para la matriz de probabilidad resultante de la figura 57, tomando en consideración que el número de casos posibles es 7 y el número de casos favorables varía en función de la parada origen destino, mostraremos a continuación el proceso de cálculo de la matriz de probabilidad. 


$$
\begin{gathered}
P_{[\text {Matriz } O D]}=\frac{\text { Número de casos favorables matriz } O D}{\text { Número de elementos matriz } O D} \\
P_{[1,8]}=\frac{2}{7}=0,29 \\
P_{[2,10]}=\frac{2}{7}=0,29 \\
P_{[3,10]}=\frac{1}{7}=0,14 \\
P_{[5,13]}=\frac{1}{7}=0,14 \\
P_{[10,11]}=\frac{1}{7}=0,14
\end{gathered}
$$

A continuación, se analiza la tabla de probabilidad, lo cual indica que la probabilidad de que el autobús suba pasajeros en la parada №1 y los baje en la parada №8 es de 0,29 (3), al igual que desde las paradas №2 hasta la №10 (4). Así mismo la probabilidad que el autobús suba pasajeros en las paradas №3, №5, №10 y se bajen en las paradas №10, №13, №11 es de 0,14 tal como se muestra en las funciones (5), (6) y (7). Lo que visualmente se vería representado como se muestra en la figura 58.

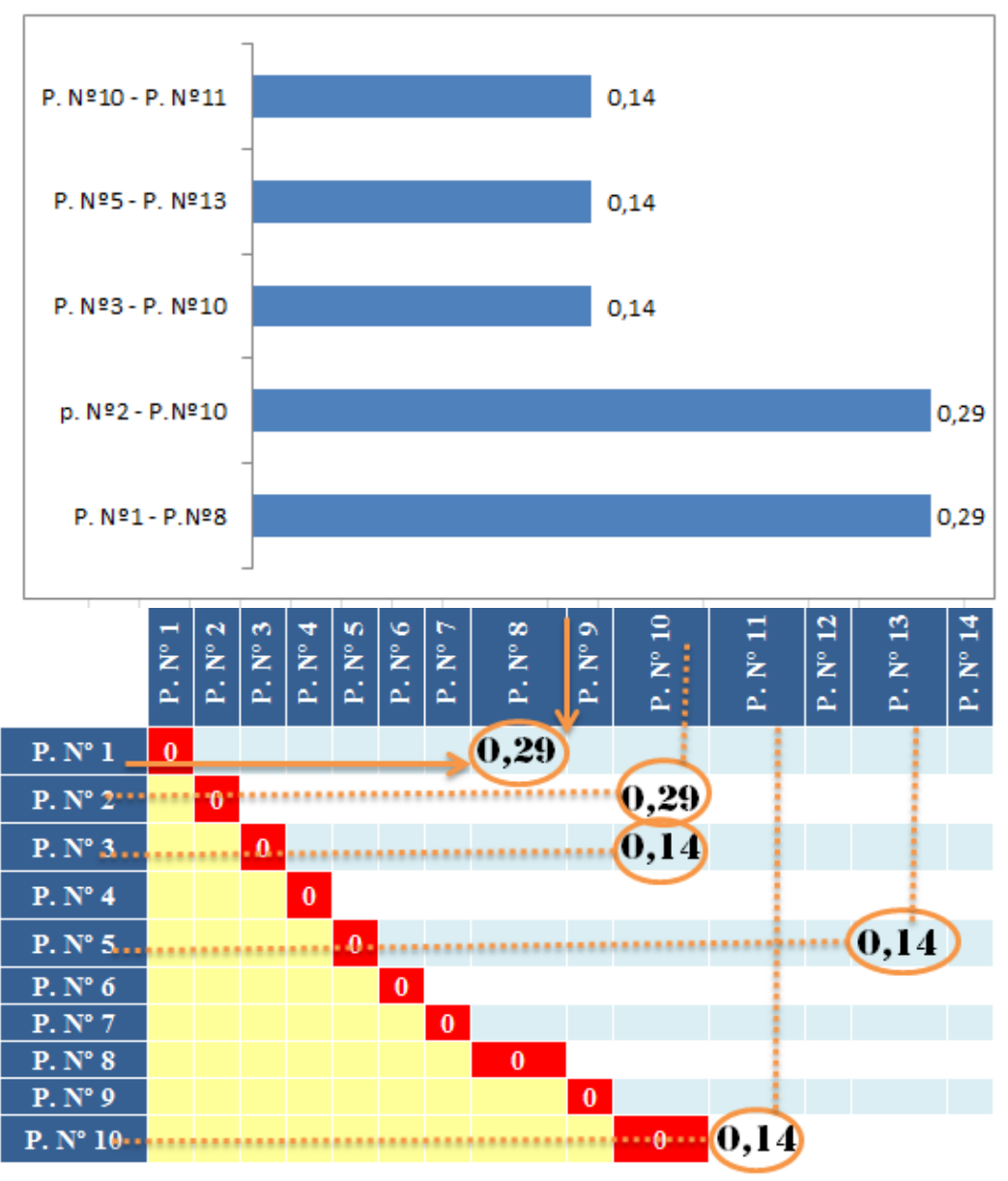

Figura 58. (a) Matriz de probabilidad, (b) Gráfica de probabilidad. 


\subsection{Tipos de informes del sistema.}

Para la elaboración de los informes del sistema se ha tomado una muestra de 15 archivos tipo texto (ver figura 59) que corresponden al recorrido del autobús desde la parada №6 hasta la parada №21. Estos archivos han sido procesados de forma manual para posteriormente comprobarlos de forma automática con el sistema.

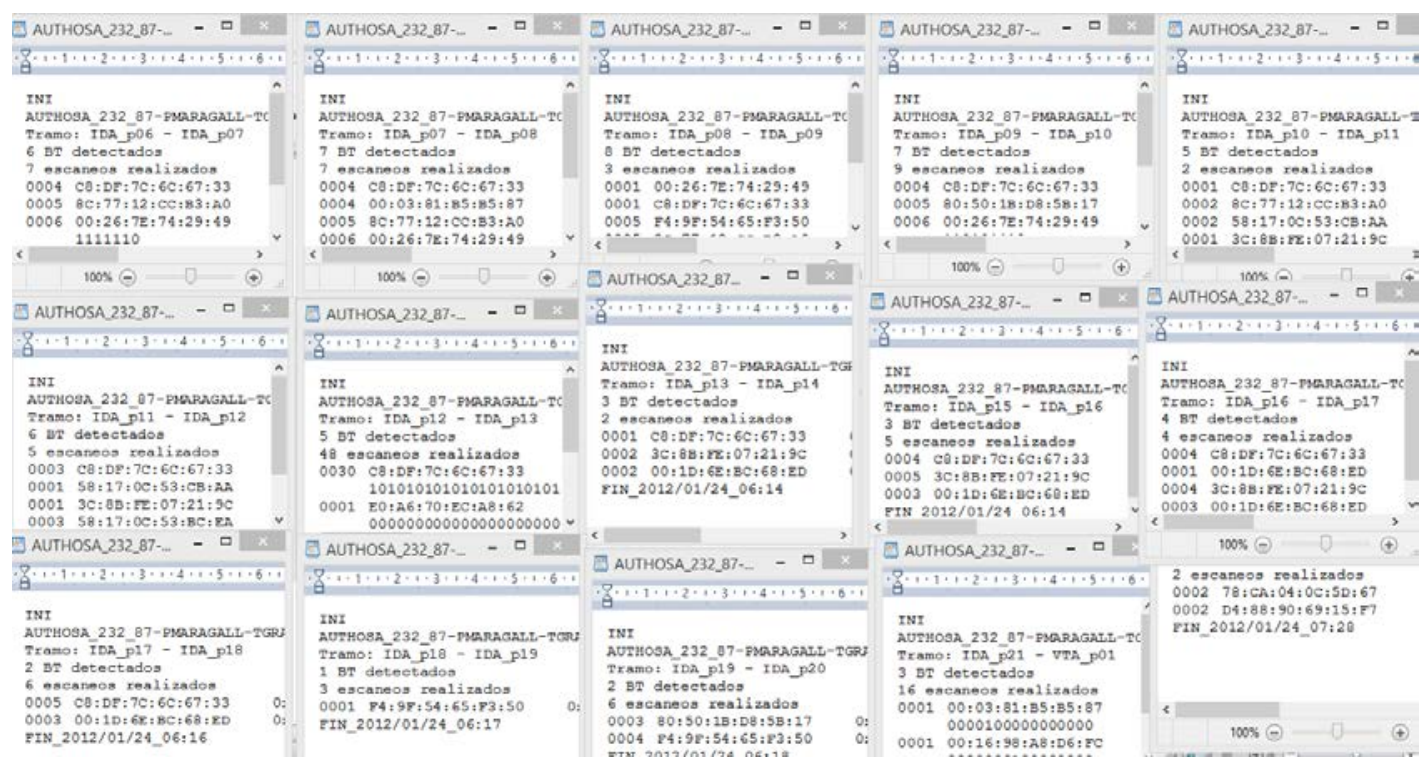

Figura 59. Documentos procesados.

Una vez elegidos los archivos pasamos a seleccionarlos para lograr un esquema de resultados coherentes según la información seleccionada. Se debe tener en cuenta que la información en este caso se procesa verificando el estado de la dirección MAC, que es la que representa a cada pasajero, y su recorrido (figura 60). 

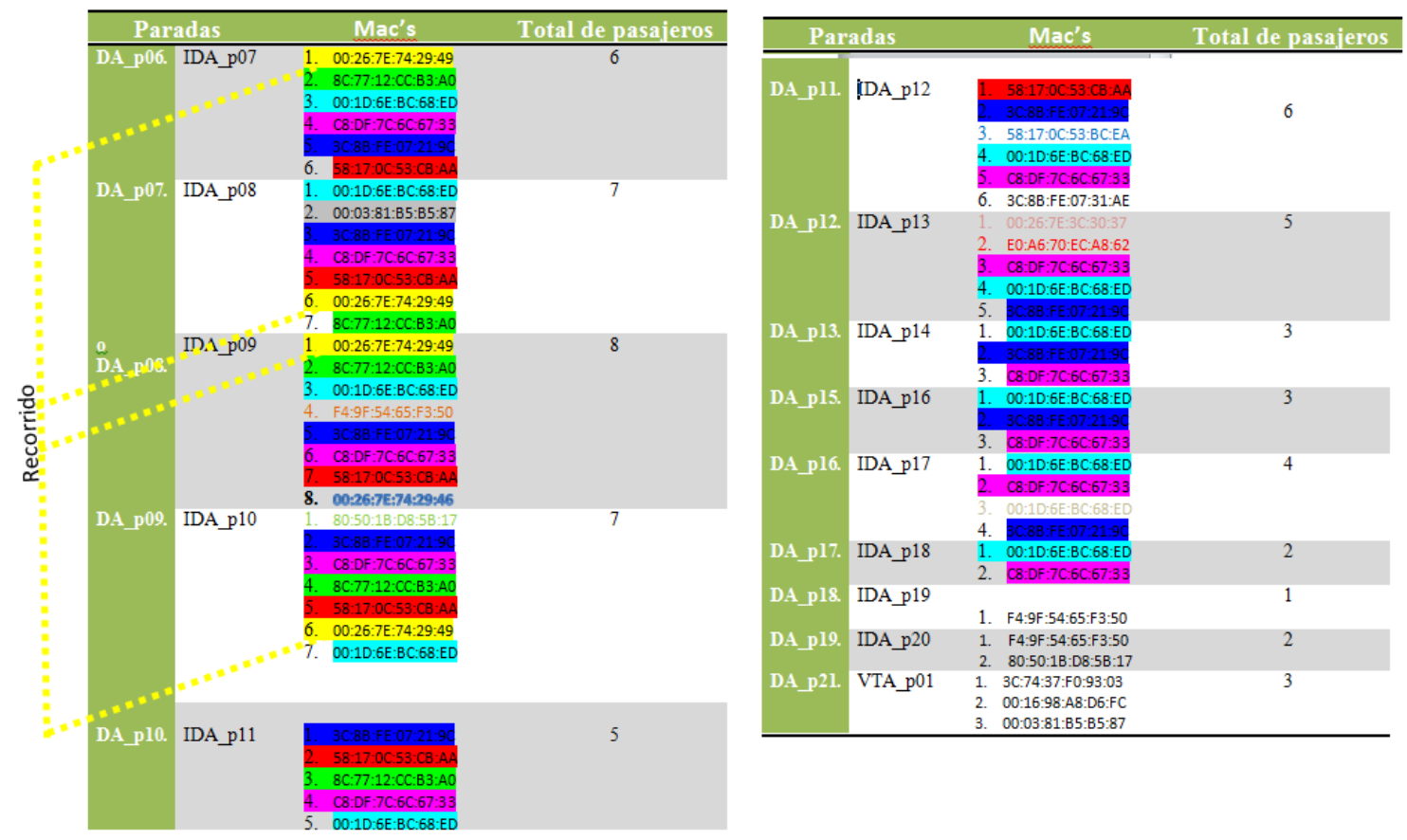

Figura 60. Tabla de Pasajeros (direcciones MAC) y su recorrido.

En la anterior figura, se puede distinguir a cada pasajero por un color por ejemplo el pasajero (00:26:7E:74:29:49 color amarillo) realiza el siguiente recorrido: Sube en la parada №6 y se baja en la №10 tal como lo representa la figura 60. Y de la misma manera para todas los pasajeros siguientes.

A partir de aquí, se puede verificar que el proceso funcione adecuadamente desde la opción reporte del menú principal (ver figura 61), mostrando otro menú donde seleccionar si deseamos la matriz O-D por fechas o por horas (ver figura 62).

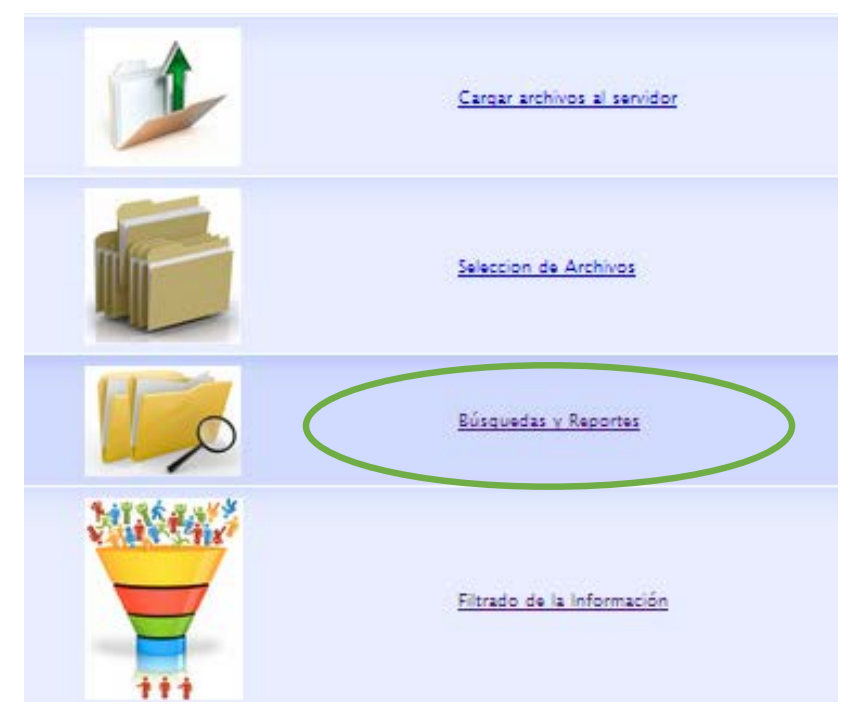

Figura 61. Selección de la opción reporte del menú principal 


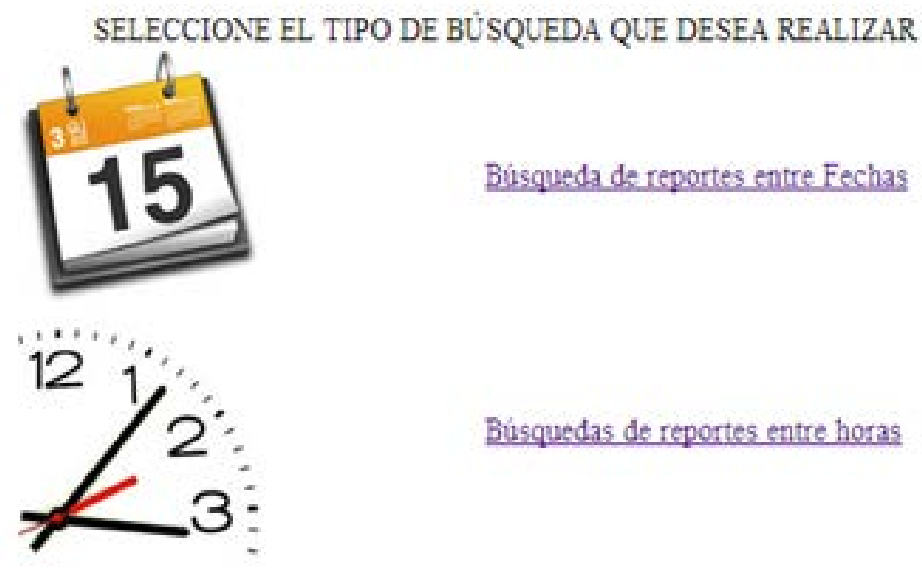

Figura 62. Sub-menú de Reportes

Para el ejemplo planteado se selecciona un reporte por fechas, es decir, una matriz 0-D por intervalos de fechas, por lo que se debe introducir las fechas para las cuales se necesita la matriz y se presiona el botón búsqueda por fecha tal como lo muestra la figura 63.

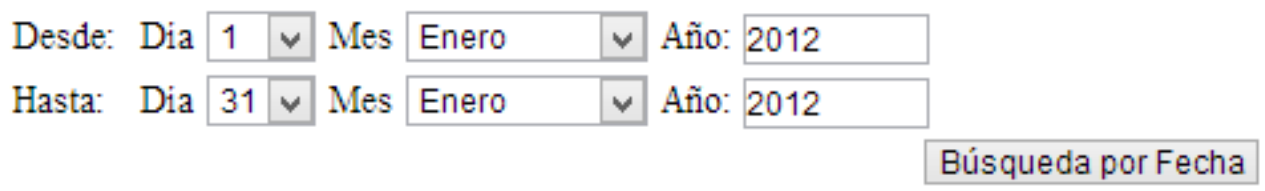

Figura 63. Pantalla del sistema para búsqueda por fechas.

Si se han realizado correctamente los pasos anteriores se obtendrá la matriz O-D tal como se muestra en la figura 64.

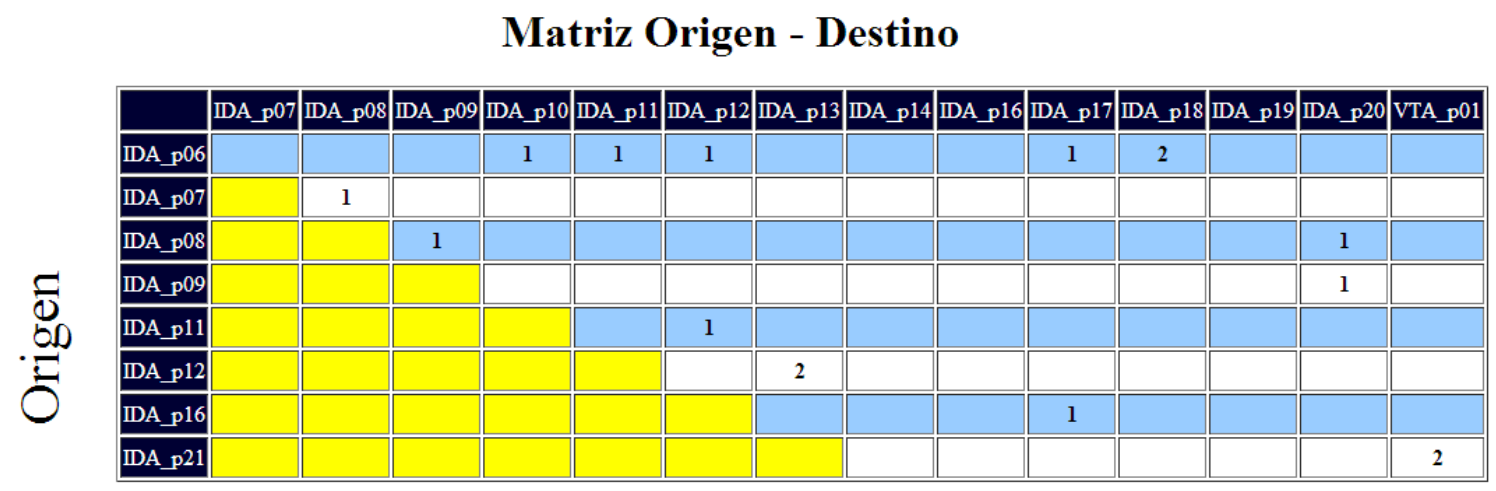

Figura 64. Matriz 0-D. 
El último paso es obtener la matriz de probabilidades, que generará la matriz de probabilidad en base a las condiciones establecidas anteriormente. Para ello, basta con pulsar el botón "ver matriz de probabilidad" (ver figura 65).

Matriz Origen - Destino

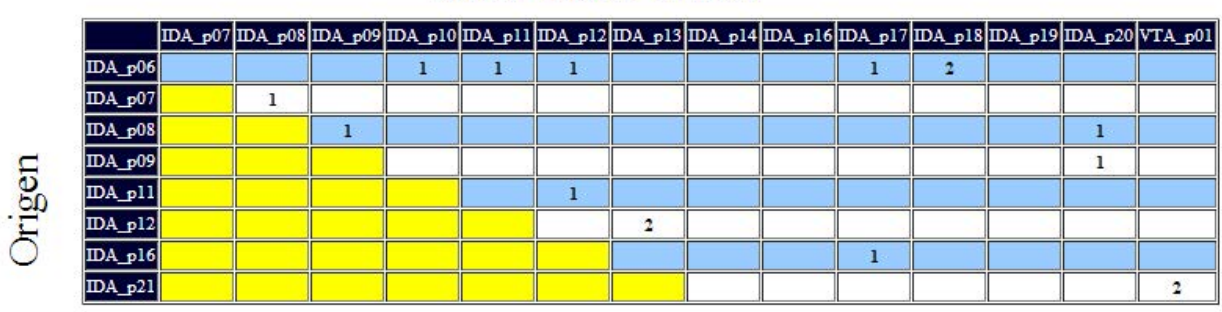

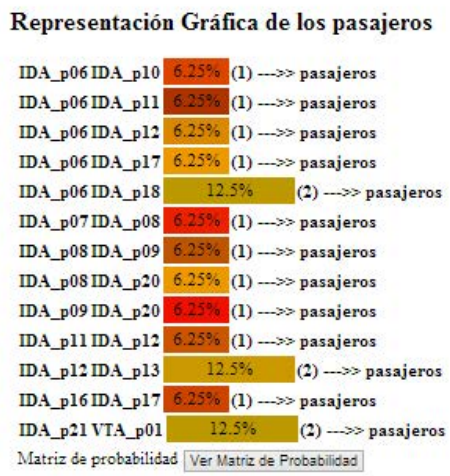

\begin{tabular}{|c|c|c|c|c|c|c|c|c|c|c|c|c|c|c|}
\hline & IDA_p07 & IDA_p08 & IDA_p09 & IDA_p10 & IDA_p11 & IDA_p12 & IDA_p13 & IDA_p14 & IDA_p16 & IDA_p1 & IDA_p18 & 8 IDA_p19 & IDA_p20 & VTA_p01 \\
\hline IDA_p06 & & & & 0.06 & 0.06 & 0.06 & & & & 0.06 & 0.13 & & & \\
\hline DA_p07 & & 0.06 & & & & & & & & & & & & \\
\hline IDA_pos & & & 0.06 & & & & & & & & & & 0.06 & \\
\hline DA_p09 & & & & & & & & & & & & & 0.06 & \\
\hline IDA_p11 & & & & & & 0.06 & & & & & & & & \\
\hline IDA_p12 & & & & & & & 0.13 & & & & & & & \\
\hline DA_p16 & & & & & & & & & & 0.06 & & & & \\
\hline IDA_p21 & & & & & & & & & & & & & & 0.13 \\
\hline
\end{tabular}

Figura 65. Pantalla del sistema Presentando la matriz 0-D

Como resultado final se obtiene la matriz de probabilidad que ha sido generada según el teorema de Laplace (figura 66).

Matriz de Probabilidad

Gráfica de Matriz de Probabilidad

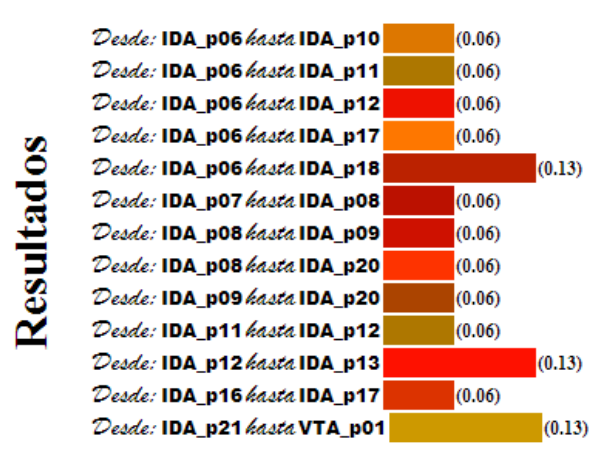

Figura 66. Matriz de probabilidad 0-D. 


\section{CAPÍTULO 4: Sistema alternativo de comunicaciones entre equipos embarcados de las líneas de transporte público}

Las Redes malladas inalámbricas (WMN) han suscitado una gran atención en los últimos años debido a sus ventajas a través del uso delas redes inalámbricas tradicionales con o sin movilidad. Una WMN puede ser vista como una mezcla de redes ad hoc y de infraestructura, con todos los beneficios de dicha arquitectura híbrida. Una red de este tipo mantiene el nivel de señal en largas distancias mediante una serie de saltos más cortos a través de nodos intermedios que no sólo regeneran la señal sino que actúan cooperativamente y de forma inteligente para ampliar la cobertura de la red y mejorar sus prestaciones. Este capítulo presenta los principales aspectos de diseño y funcionamiento de un protocolo o procedimiento nuevo de conexión que permite la extensión de la cobertura en este tipo de redes para la transmisión de mensajes de datos. El procedimiento incluye mensajes reconocimiento o confirmación de recepción de la información en el nodo de destino $y$, adicionalmente, proporciona un mecanismo de ahorro de energía, para que los nodos móviles intermedios que funcionan principalmente como gateways reenviando datos desde o hacia los nodos vecinos, no agoten sus baterías.

\subsection{Introducción}

Actualmente toda la sociedad está integrada en la llamada sociedad de la información, que según una de las definiciones de la U.E. es "Una sociedad en la que la creación, distribución y manipulación de la información forman parte importante de las actividades culturales y económicas". No hay que mirar lejos para observar que diariamente se utiliza la tecnología para comunicarse en todos los diferentes tipos de ámbito social, todas las personas utilizan tecnologías de comunicación casi sin darse cuenta en su quehacer diario: cuando aceptan una llamada con el teléfono móvil o fijo, se consulta el correo electrónico, durante el chat, en la extracción o consulta de dinero en un cajero automático o accesos y consultas a servicios mediante PDA, tablets, móvil o incluso plataformas portables de videojuegos.

Por otro lado, también tenemos software y comunicaciones en todo tipo de útiles diarios: desde el coche o la calefacción de casa hasta los juguetes de los niños. Todos estos dispositivos son susceptibles de intercomunicarse entre sí y, aunque 
pueden hacerlo por los sistemas de cables tradicionales, su mayor potencial se alcanza a través de las comunicaciones inalámbricas.

Multitud de estos aparatos electrónicos utilizan hoy en día diferentes sistemas inalámbricos con tendencia a utilizar más de una interfaz inalámbrica en un mismo dispositivo para lograr la máxima interconectividad posible entre dispositivos.

Las tecnologías inalámbricas más extendidas en la actualidad son las tecnologías WiFi, Bluetooth, red GSM/3G, infrarrojos, WiMAX... Las ventajas principales de las tecnologías inalámbricas son fundamentalmente su base en estándares internacionales, algunas operan en bandas libres de frecuencia, permiten aplicaciones de alta capacidad por encima de los 50Mbps a costes razonables tanto en interior como exterior. Mientras que los dos inconvenientes más importantes son la seguridad e integridad de los datos transmitidos y la limitación de alcance por falta de cobertura.

El protocolo descrito en este apartado aborda un tema muy actual relacionado con la problemática de la cobertura en los servicios inalámbricos. En multitud de ocasiones cuando se hace uso de la tecnología inalámbrica es apreciable que incluso con todos los avances actuales que permiten tener una gran experiencia multimedia o una comunicación perfecta con otra persona situada en cualquier lugar; las cuales hacen uso de las últimas tecnologías de codificación, multiplexación, cifrado... son inútiles ante el simple hecho de que se esté en un punto sin cobertura inalámbrica a pesar de que existen ocasiones donde desplazarse una pequeña distancia permita hacer uso de todas estas tecnologías.

La reciente emergencia desde un punto de vista económico de las comunicaciones inalámbricas así como de los dispositivos informáticos portátiles, junto con los avances en infraestructuras de comunicación ha permitido un gran crecimiento de las redes móviles. Este auge prácticamente exponencial de las redes celulares se ha basado en una combinación de tecnologías cableadas e inalámbricas.

Sin embargo, el interés tanto de la industria como de la comunidad científica en el campo de las telecomunicaciones ha cambiado recientemente hacia el desarrollo de sistemas móviles donde algunas partes de la red carecen de infraestructura fija. En este sentido, las redes ad hoc se han convertido en la 
tecnología de vanguardia en las redes de comunicaciones móviles. De hecho, estas redes constituyen el primer paso para proporcionar soluciones a un coste razonable con anchos de banda dinámicos en áreas de cobertura específicas, ya que permiten la interconexión de los nodos de la red usando directamente transceptores inalámbricos (por lo general a través de caminos con múltiples saltos) sin la existencia de una infraestructura fija. Esta es una característica muy distintiva de las redes ad hoc en comparación con redes inalámbricas tradicionales como las celulares o inalámbricas de área local (WLAN), donde los nodos se comunican entre sí sólo a través de las estaciones fijas y conocido como modo infraestructura.

Las redes malladas inalámbricas (WMN) combinan la mezcla de redes tipo infraestructura con redes ad hoc. De esta forma y partiendo de un comportamiento base tipo infraestructura, se posibilita la conexión de dispositivos fuera del alcance del punto de acceso o estación base, a través de dispositivos que de forma directa o indirecta están dentro del alcance de cobertura del punto de acceso. Actualmente, el cuello de botella de esta tecnología se encuentra en el protocolo adecuado que posibilite dicha conexión, la eficiencia energética de la comunicación y el consumo energético de los nodos. Por ello la propuesta y desarrollo de protocolos de comunicación energéticamente eficientes es sin duda una gran contribución al desarrollo global de la tecnología.

En los últimos años las WMN se han convertido en un campo de gran actividad investigadora debido a su naturaleza, como ya se ha comentado, y su potencial de aplicaciones en múltiples escenarios [Zou'2010], [Yu'2010]. Además pueden desplegarse en teoría, sobre casi cualquier tecnología inalámbrica existente como WIFI, WIMAX, 3G, LTE, etc. y coexistir [Ghazisaidi'2009]. Por lo que, a pesar de que el concepto híbrido celular/ad hoc no es nuevo [Wu'2001], [Luo'2003], se le presume un aspecto ubicuo y una línea de gran interés e investigación en los próximos años.

De hecho, estamos siendo testigos del progreso continuo en técnicas de enrutamiento, protocolos basados en la asignación de canal y el aumento de las tasas de transferencia en las WMN [Kim'2011], [Pediaditaki'2009]. Asimismo, la implementación de protocolos proactivos, reactivos e híbridos para la optimización de los tráficos en la red también está atrayendo gran atención, como en [Kum'2010]. 
Sin embargo, hay varios aspectos relacionados con la optimización de la disposición los recursos en términos de ahorro y consumo de energía en las WMN que actualmente representan un desafío para la comunidad científica. Por lo que, aunque problemas más complejos de las WMN como los relacionados con el comportamiento de los nodos [Martignon'2009] o la seguridad [Lee'2009] están siendo adecuadamente tratados, los problemas relacionados con el consumo de energía siguen siendo en la actualidad un gran freno para esta tecnología. El protocolo presentado en este trabajo aporta luz suficiente para abordar el despliegue de redes WMN con extensión de cobertura atendiendo las necesidades de ahorro de energía requeridas [Cerdan'2014] y contribuyendo por tanto a la planificación eficiente de rutas en líneas de transporte público tanto por el ahorro de costes en comunicaciones como por el uso de redundancia en las comunicaciones de interés para la planificación de la flota.

\subsection{Escenario de referencia}

En el escenario inicial (figura 67) se define como núcleo de red a un equipo único (servidor, punto de acceso o estación base) que supervisará en todo momento el funcionamiento de la red. Dispondrá de una interfaz inalámbrica y será el responsable de crear y mantener una red en modo infraestructura a la que se conectarán los diversos nodos que se agreguen a la red. Este equipo se comunicará con los terminales móviles haciendo uso de una red con topología punto a multipunto o también llamada red en modo infraestructura. Todo el tráfico generado por los nodos se dirigirá siempre a este equipo, siendo así posible el control y tarificación de todos los envíos de datos entre terminales móviles.

Asimismo se define como nodo o terminal móvil, a un equipo portátil y que esté alimentado por baterías, por lo que estará claramente definido por una baja capacidad de procesamiento y unos recursos energéticos limitados. El número máximo de nodos por red en principio no es limitado, quedando posteriormente limitado por la implementación y administración por parte del núcleo de red de los nodos. Un nodo dispondrá de dos interfaces inalámbricas, utilizándose una de ellas para la conexión directa con el núcleo de red y otra para que los terminales se comuniquen entre ellos haciendo uso de una red con una topología de nodos multipunto o también llamada red ad hoc. 
Como se ha indicado cada nodo de la red tendrá acceso a dos redes inalámbricas, una configurada en modo infraestructura con el servidor, y otra en modo ad hoc con conexión directa con los nodos vecinos de su alrededor.

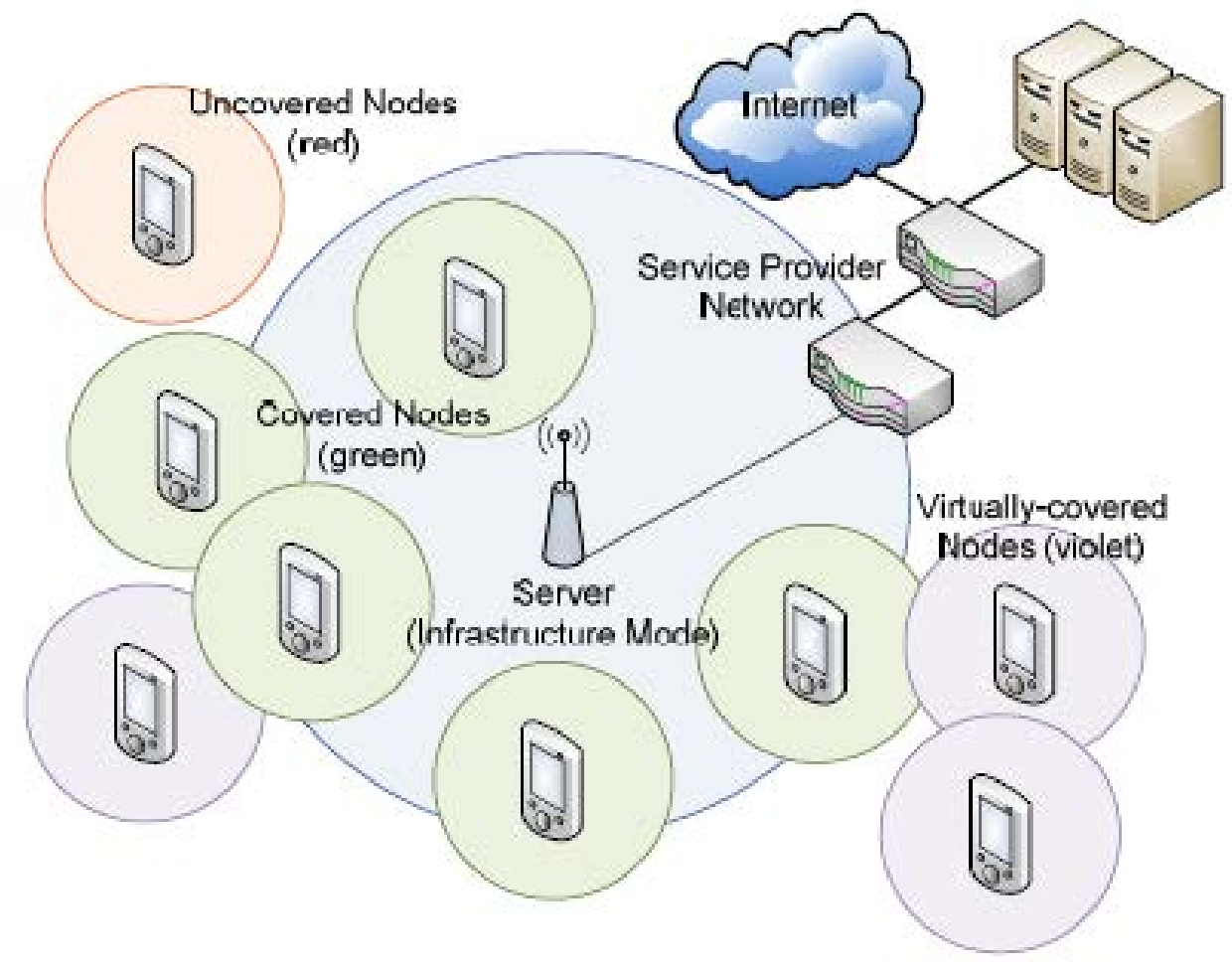

Figura 67. Entorno de referencia para el funcionamiento del protocolo

Para que este escenario sea implementado correctamente, se supone que los nodos que tienen acceso al servicio están motivados para actuar desinteresadamente [Martignon'2009]. Por lo que se considerará que los usuarios cooperan favoreciendo el correcto funcionamiento del protocolo y que en la práctica se traduce en que el usuario tiene activado por defecto en su terminal ciertos componentes software o hardware.

\subsection{Especificación del protocolo}

Cuando un terminal móvil genere un mensaje para cualquier otro terminal existirán dos posibilidades de realizar el envío:

- Si el terminal móvil que genera el mensaje está dentro del radio de cobertura del núcleo de red, el terminal enviará directamente el mensaje 
hacia el núcleo de red, con la consiguiente confirmación de la recepción por parte del núcleo de red.

- Sin embargo, si el terminal móvil no tiene conexión directa con el núcleo de red, difundirá el mensaje a la red por la interfaz configurada como ad hoc.

Cualquier mensaje de datos generado y enviado por un terminal móvil se direccionará siempre hacia el núcleo de red. Cuando un terminal móvil recibe un mensaje de otro terminal móvil; se limitará a actuar como una terminal pasarela, por lo que no analizará el mensaje. Simplemente se limitará a reenviar el mensaje para hacerlo llegar al servidor. Las consecuencias que se deducen de este comportamiento es que se obtiene como ventaja que se garantiza que todos los mensajes de datos serán correctamente cuantificados y tarificados por el servidor. Asimismo podemos asegurar que no habrá saturación en los nodos por esta operación tipo ad hoc, ya que los datos trasmitidos por medio de esta red serán difundidos sin ningún tipo de procesamiento o análisis. Mientras que como inconveniente se produce una mayor utilización de recursos, en comparación con el caso en que el terminal móvil analizara el mensaje y lo aceptara como propio al ir dirigido a él; evitando así su propagación hacia el servidor.

Pueden existir multitud de terminales móviles que actúen como pasarela entre el servidor y los terminales móviles origen y destino, por lo que no tiene por qué existir solamente uno.

Cuando el servidor tiene un mensaje para algún nodo, difunde un mensaje de testeo para conocer si el nodo destinatario está dentro de su área de cobertura.

- En caso afirmativo se procederá al envió selectivo del mensaje hacia el nodo destinatario, contestando este último con un mensaje de confirmación en la recepción.

- En caso contrario se realizará una búsqueda del destinatario haciendo uso de la red ad hoc creada por los nodos mediante la técnica de múltiples saltos. Existiendo dos posibles casos:

o Si el destinatario es localizado, el servidor enviará los datos haciendo uso del mecanismo de múltiples saltos. 
o Si el destinatario no es localizado, el servidor almacenará los datos para su posterior reintento.

Existen dos estrategias para el envío de los mensajes en los casos en los que el núcleo de red necesite utilizar múltiples saltos para llegar a nodos que son inaccesibles por comunicación directa:

- Opción A. Cuando se realice la localización del terminal móvil, conforme se vaya encaminando el mensaje de respuesta hacia el servidor ir almacenando la ruta (la cual va recorriendo el mensaje) dentro del mensaje de respuesta. Para que a continuación el servidor envíe el mensaje de datos por esa misma ruta. Con esto se obtiene una mayor optimización de los recursos energéticos, por el contrario se produce una comunicación más inestable debido a que si cualquiera de los nodos utilizados para encaminar el mensaje hacia el destino se desplaza significativamente, la conexión será infructuosa. Esto producirá que el mecanismo de envío utilizado por el protocolo deba realizar todos los pasos anteriores para intentar transmitir el paquete de nuevo. Este problema es frecuente en redes con una velocidad movilidad muy alta, como puede ser el caso que los terminales móviles estén dentro de vehículos.

- Opción B. Cuando se realice la localización del terminal móvil destinatario, ignorar la ruta por la cual se accedió a él. Por lo que el mensaje de respuesta solamente nos indicará la existencia o no de la conectividad con el terminal móvil destino. Con ello se obtiene un desaprovechamiento energético de la red debido a que el mecanismo de difusión del mensaje afectará a un número mayor de dispositivos. Sin embargo se obtiene la ventaja de asegurar con alta probabilidad la recepción del mensaje por parte del destinatario, independientemente del tipo de red que sea, ya que será un protocolo más flexible a cambios en la red.

El protocolo presentado en este apartado utiliza la opción B como estrategia, favoreciendo en este caso la fiabilidad sobre el rendimiento energético. 
Adicionalmente el protocolo incluye un mecanismo de ahorro de energía que actúa sobre los nodos que actúan de pasarela difundiendo mensajes de otros nodos hacia o desde el servidor al tener una localización cercana al borde del área de cobertura de la red en modo infraestructura. Este mecanismo consiste en anular la función de reenvío o difusión de mensajes en función del valor de un contador de reenvíos y la carga actual de la batería del nodo.

Finalmente se utilizan dos mecanismos para evitar el colapso de la red:

- Cada mensaje incorpora un contador de saltos, limitando el número de saltos que puede dar.

- Cada mensaje incorpora un identificador único que evitará que un mismo terminal reenvíe varias veces el mismo mensaje, evitando así bucles infinitos.

\subsection{Descripción del funcionamiento en cada caso de uso}

A continuación se mostrará el intercambio de mensajes del protocolo ante diversas localizaciones de los terminales móviles respecto al servidor. Se hará referencia al terminal que genera el mensaje como "Cliente origen" y al terminal destinatario como "Cliente destino". En algunos casos aparece un terminal denominado como "Cliente pasarela", debido a que actúa como pasarela entre los terminales y el servidor.

\subsubsection{Caso 1}

Un terminal móvil quiere establecer una conexión hacia otro terminal, existiendo sendas conexiones directas con el núcleo de red. Considerándose independiente la conexión directa entre los terminales. 


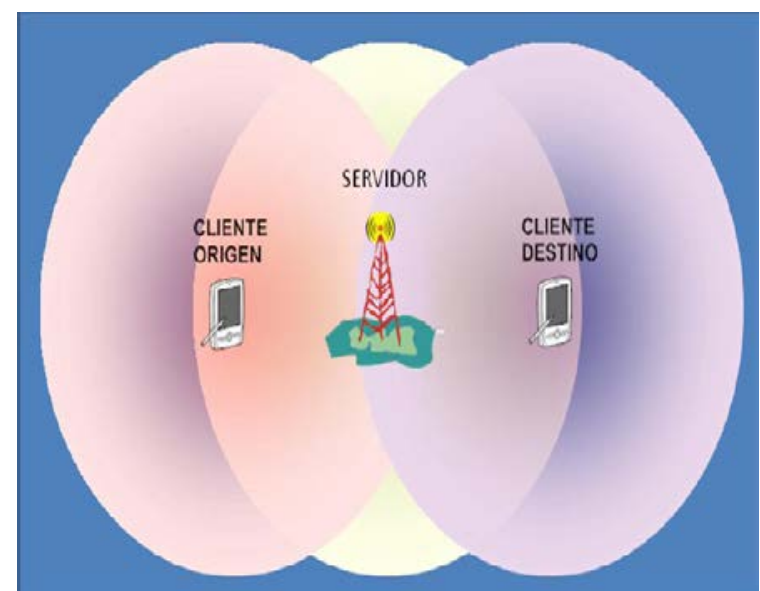

Figura 68. Escenario caso 1

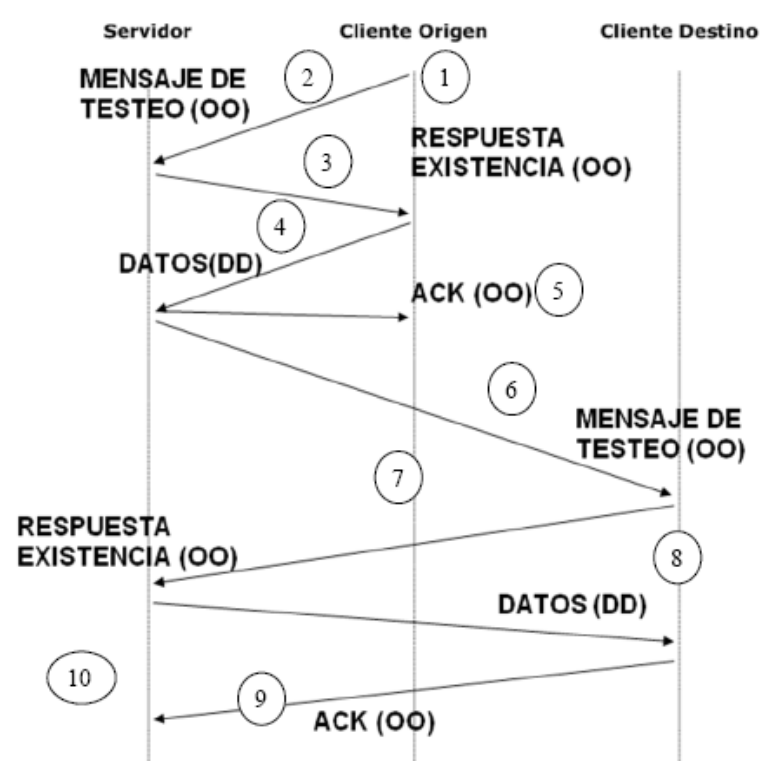

Figura 69. Intercambio de mensajes caso 1

1. Un terminal genera un mensaje de datos para otro terminal perteneciente a la red.

2. El terminal envía un mensaje de testeo para conocer la disponibilidad del servidor.

3. El servidor responde al mensaje de testeo.

4. El cliente envía el mensaje de datos hacia el servidor; puesto que tiene visibilidad directa con este; se lo envía al servidor sin utilizar ningún terminal como intermediario.

5. El servidor almacena los datos recibidos.

6. El servidor envía un mensaje de testeo para conocer la disponibilidad del cliente destino.

7. El cliente destino envía un mensaje de existencia para darse a conocer al servidor.

8. El servidor envía el mensaje de datos hacia el cliente destino.

9. El cliente destino envía un mensaje ACK (reconocimiento) al servidor para informarle que se recibieron los datos.

10. El servidor elimina los datos almacenados en su memoria. 


\subsubsection{Caso 2}

Un terminal móvil quiere establecer una conexión hacia otro terminal, existiendo una conexión directa desde el terminal generador con servidor. El terminal destinatario no tiene conexión directa con el servidor, pero sin embargo puede realizar una conexión utilizando otros terminales como intermediarios, donde uno o más de ellos disponen de conexión directa con el servidor.

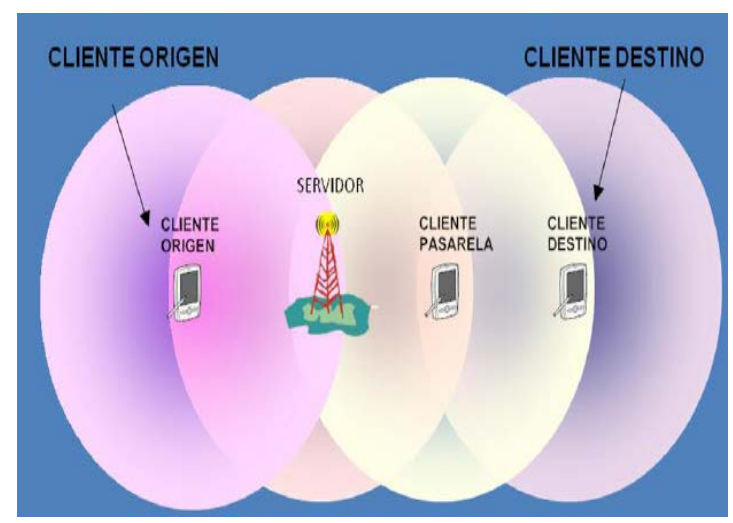

Figura 70. Escenario caso 2

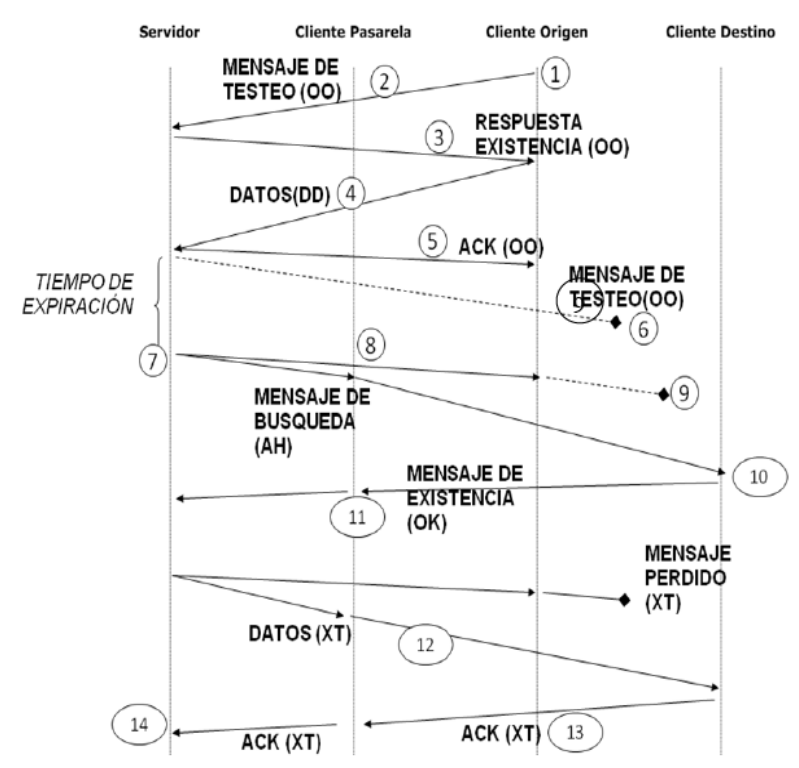

Figura 71. Intercambio de mensajes en el caso 2

1. Un terminal genera un mensaje de datos para otro terminal perteneciente a la red.

2. El terminal envía un mensaje de testeo para conocer la disponibilidad del servidor.

3. El servidor responde al mensaje de testeo.

4. El cliente envía el mensaje de datos hacia el servidor; puesto que tiene visibilidad directa con este; se lo envía al servidor sin utilizar ningún terminal como intermediario.

5. El servidor almacena los datos y comienza la búsqueda del cliente destino.

6. Expira el tiempo máximo de espera en el que el cliente destino debería haber contestado al mensaje de testeo enviado por el servidor. 
7. El servidor inicia la búsqueda del cliente destino mediante mensaje de búsqueda. Estos mensajes se propagan por la red utilizando el mecanismo de múltiples saltos.

8. El cliente pasarela y el cliente origen reciben el mensaje de búsqueda. Estos clientes a su vez reenvían el mensaje y lo registran para evitar reenviarlo de nuevo. Cada vez que se envían estos mensajes, se decrementa el contador de saltos en una unidad.

9. El cliente origen no consigue transmitirlo a ningún otro cliente.

10. El mensaje de búsqueda emitido por el cliente pasarela llega al cliente destino

11. El cliente destino envía un mensaje especial de existencia, el cual se propaga por la red utilizando el mecanismo de múltiples saltos.

12. El servidor envía los datos hacia el cliente destino, haciendo uso nuevamente del multihopping.

13. El cliente destino envía un mensaje especial ACK, el cual se propaga mediante multihopping. Hasta llegar al servidor.

14. El servidor elimina los datos almacenados en memoria.

\subsubsection{Caso 3}

Un terminal móvil quiere establecer una conexión hacia otro terminal. En este caso no existe una conexión directa desde el terminal generador con servidor de red, sin embargo puede realizar una conexión utilizando otros terminales como intermediarios, donde uno o más de ellos disponen de conexión directa con el servidor.

En este caso el terminal destinatario si dispone de conexión directa con el servidor. 


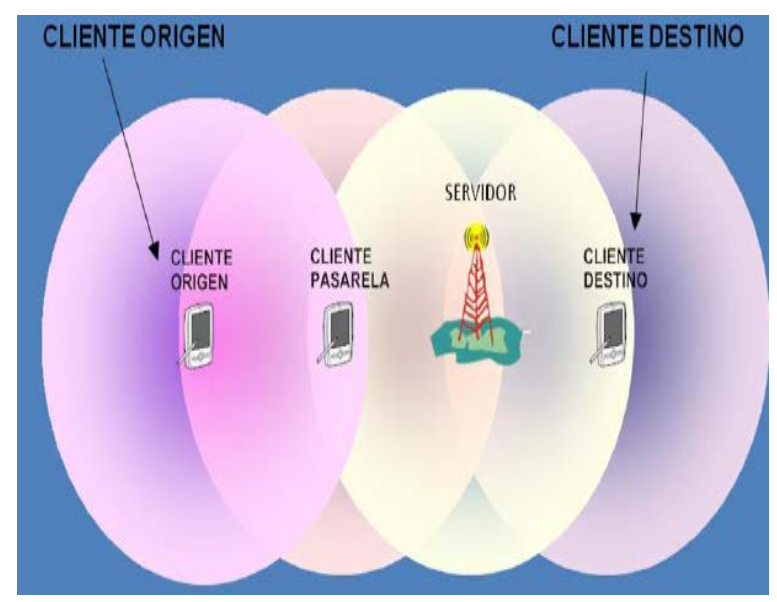

Figura 72. Escenario caso 3

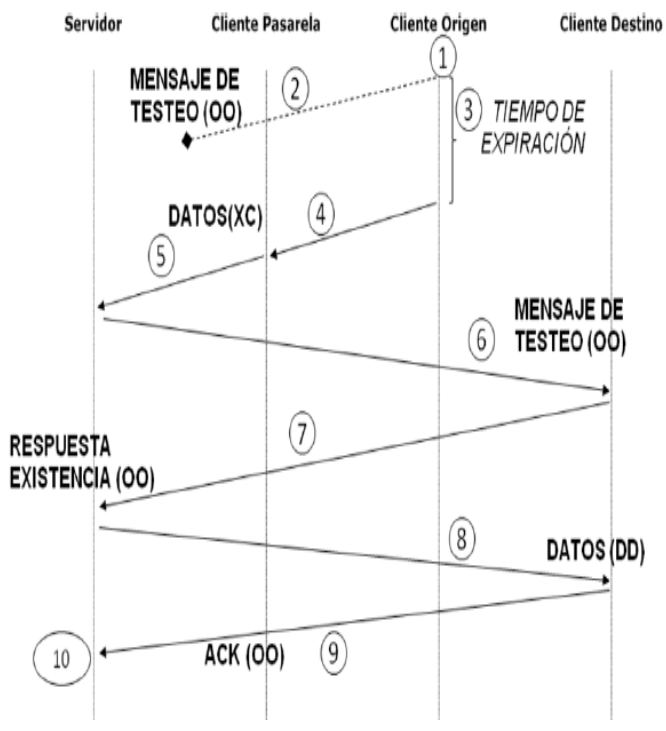

Figura 73. intercambio de mensajes en el caso 3

1. Un terminal genera un mensaje de datos para otro terminal perteneciente a la red.

2. El terminal envía un mensaje de testeo para conocer la disponibilidad del servidor.

3. Expira el tiempo máximo de respuesta por parte del servidor, debido a que el cliente origen no dispone de conexión directa con el servidor

4. El terminal envía su mensaje de datos mediante múltiples saltos, llegando el mensaje al cliente pasarela. El cliente origen desconocerá si el mensaje fue recibido por el servidor, debido a que no se producirá un mensaje de respuesta desde el servidor.

5. El cliente pasarela reenvía el mensaje automáticamente, llegando este al servidor

6. El servidor almacena los datos y comienza las búsqueda del cliente destino

7. El cliente destino envía un mensaje de existencia para darse a conocer al servidor

8. El servidor envía el mensaje de datos hacia el cliente destino.

9. El cliente destino envía un reconocimiento al servidor para informar que se recibieron los datos.

10. El servidor elimina los datos almacenados de su memoria. 


\subsubsection{Caso 4}

Un terminal móvil quiere establecer una conexión hacia otro terminal. En este caso no existe una conexión directa ni desde el terminal generador ni desde el terminal destinatario con el servidor de red, sin embargo ambos terminales pueden realizar una conexión utilizando otros terminales como intermediarios, donde uno o más de ellos disponen de conexión directa con el servidor.

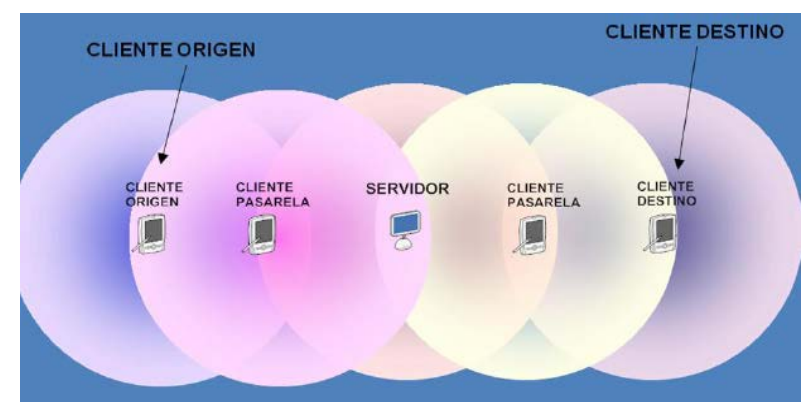

Figura 74. Escenario caso 4

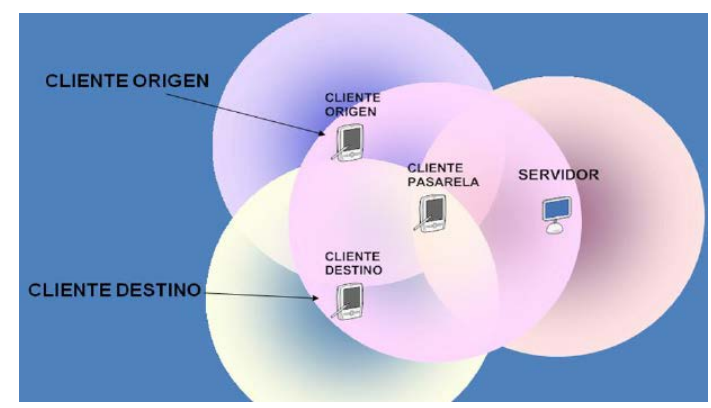

Figura 75. Escenario caso 4 alternativo

A pesar de la divergencia que existe entre las situaciones de la figura 74 y la figura 75, el sistema de funcionamiento será exactamente el mismo. La única diferencia reside en utilizar un cliente pasarela distinto para el cliente destino y el cliente origen (figura 74) o bien utilizar un cliente pasarela común para ambos (figura 75).

Este caso se puede resolver como un caso combinado del caso 2 y 3; anteriormente explicados; ya que ambos clientes no tienen acceso directo con el servidor. En este caso omitimos la ilustración del intercambio de mensajes aunque los enumeramos a continuación:

1. Un terminal genera un mensaje de datos para otro terminal perteneciente a la red.

2. El terminal envía un mensaje de testeo para conocer la disponibilidad del servidor.

3. Expira el tiempo máximo de respuesta por parte del servidor, debido a que el cliente origen no dispone de conexión directa con el servidor

4. El terminal envía su mensaje de datos mediante múltiples saltos, llegando el mensaje al cliente pasarela. El cliente origen desconocerá si el mensaje 
fue recibido por el servidor, debido a que no se producirá un mensaje de respuesta desde el servidor.

5. El cliente pasarela reenvía el mensaje automáticamente, llegando este al servidor

6. El servidor almacena los datos y comienza la búsqueda del cliente destino.

7. Expira el tiempo máximo de espera en el que el cliente destino debería haber contestado al mensaje de testeo enviado por el servidor.

8. El servidor inicia la búsqueda del cliente destino mediante mensaje de búsqueda. Estos mensajes se propagan por la red utilizando el mecanismo de múltiples saltos.

9. El cliente pasarela y el cliente origen reciben el mensaje de búsqueda. Estos clientes a su vez reenvían el mensaje y lo registran para evitar reenviarlo de nuevo. Cada vez que se envían estos mensajes se decrementa el contador de saltos en una unidad.

10. El cliente origen no consigue transmitirlo a ningún otro cliente.

11. El mensaje de búsqueda emitido por el cliente pasarela llega al cliente destino y este o envía un mensaje especial de existencia, el cual se propaga por la red utilizando el mecanismo de múltiples saltos.

12. El servidor envía los datos hacia el cliente destino, haciendo uso nuevamente de múltiples saltos.

13. El cliente destino envía un mensaje especial ACK, el cual se propaga mediante múltiples saltos hasta llegar al servidor.

14. El servidor elimina los datos almacenados en memoria. 
4.4.5 Mejora del tráfico cursado en la red de comunicaciones con el uso del protocolo propuesto

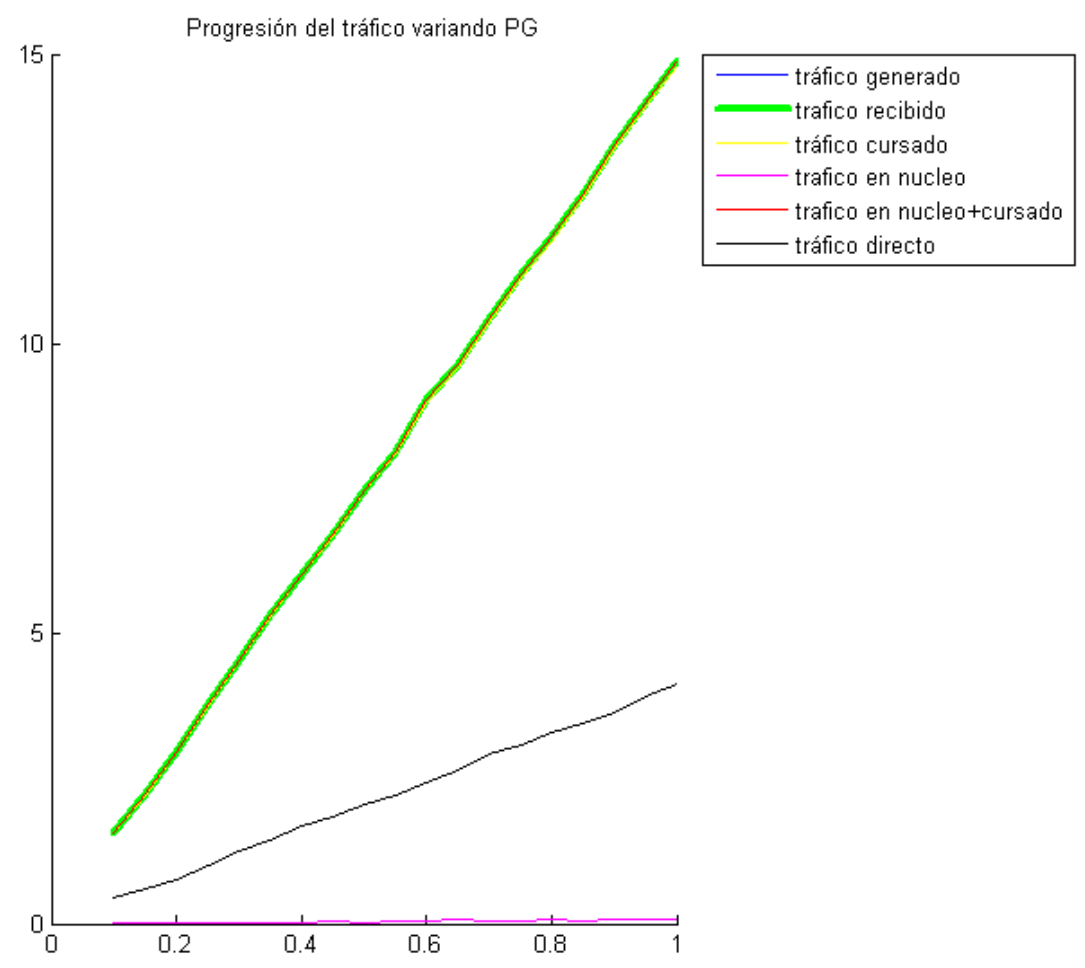

Figura 76. Progresión del tráfico variando PG

En la figura anterior se aprecia claramente que todos los tráficos aumentan linealmente con la generación de tráfico. Todo el tráfico que se genera es cursado, ya que se tiene acceso directo o indirecto con todos los terminales. También se puede apreciar el tráfico que se podría haber cursado sin la aplicación de este protocolo (línea negra). A continuación se muestra una gráfica de porcentaje del incremento de tráfico que se obtiene en esta simulación, donde llega a un máximo del $383 \%$ de incremento.

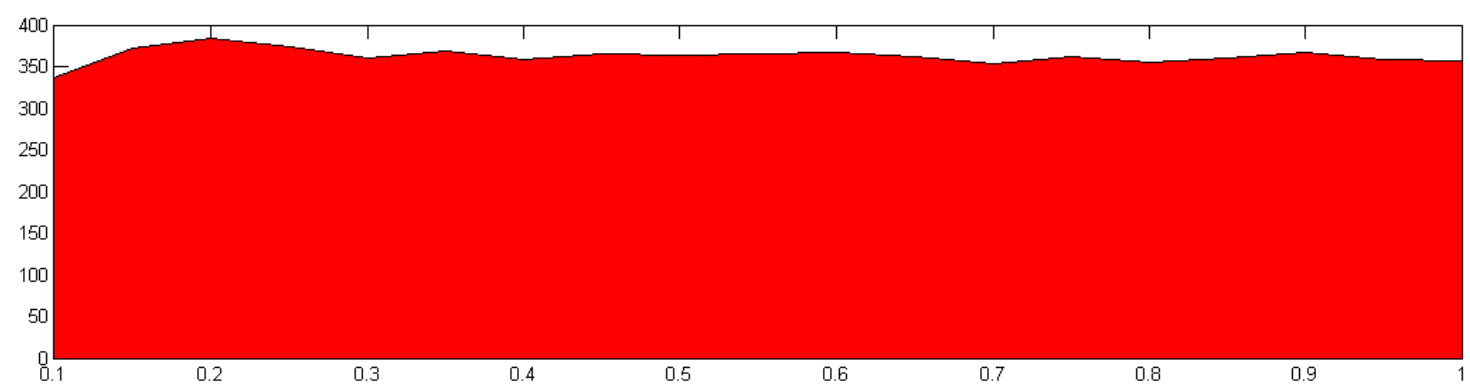

Figura 77. Porcentaje del incremento de tráfico obtenido en la simulación. 


\section{CAPÍTULO 5: Conclusiones}

El valor máximo en el ratio de ocupación de un vehículo de transporte público es de 3 a 4,5 pasajeros por metro cuadrado. Así lo establecen las normas europeas de regulación en materia de calidad del servicio prestado al viajero y deberían cumplirlo, consecuentemente, las distintas compañías de transporte que operan dicho servicio. Por eso, los nuevos Sistemas de Ayuda a la Explotación (SAE) de los que se dotan los operadores de transporte, incluyen o deberían incluir, entre otras prestaciones:

- Algoritmos de regulación avanzados y parametrizables

- Localización automática y continua

- Paneles de Información al Usuario en paradas con información diversa (tiempo de espera, ocupación...)

- Niveles de ocupación del Autobús o Metro

Así mismo, la información que proporciona el grado de ocupación por zona o el flujo del pasaje es requerida, cada vez con mayores niveles de detalle y sofisticación, por los distintos departamentos de Planificación del Servicio, de Regulación, de Validación y Venta o de Telecomunicaciones en muy diversas compañías de autobuses, metro, tranvía y ferrocarril. A grandes rasgos, no es posible regular sin conocer el estado de carga de los vehículos; no se pueden planificar las frecuencias de paso sin disponer de los patrones de uso, ni configurar el mapa de líneas si se carece de la información origen - destino de los trayectos más habituales. Para disponer de todos estos datos se requiere del concurso de distintas tecnologías en el ámbito TIC. Por ejemplo:

- El análisis de la demanda real de los ciudadanos; los cada vez más numerosos dispositivos conectados a la red; servicios públicos ajustados a las necesidades de los usuarios; smart cities que aprovechan las últimas tecnologías; movilidad y transporte sostenible mediante la aplicación de los sistemas inteligentes de transporte;

- Los servicios ofrecidos en el análisis de datos ubicuos a gran escala del comportamiento urbano del ciudadano, mediante la integración de los cuantiosos datos digitales que deja a su paso por la ciudad; 
- El modelado de este comportamiento y la recogida de las demandas de los ciudadanos, en cuanto a transporte, que se utilizarán para realimentar las tecnologías y los servicios y permitirán ajustar los servicios interactivos y accesibles ofrecidos a las necesidades reales;

- La mejora del bienestar del ciudadano mediante una red de servicios urbanos inteligentes y personalizados; nuevas herramientas tecnológicas que incentiven el uso del transporte público, uno de los principales retos para el futuro de las ciudades; nuevas soluciones, basadas en los sistemas inteligentes de transporte, que eviten las congestiones de tráfico, así como los costes directos e indirectos que suponen.

Este trabajo de tesis ha contribuido a todo ello. En primer lugar, mediante el desarrollo de un prototipo y ejecución de un piloto de validación experimental de un sistema automático de conteo de pasaje que posibilita la estimación del estado de la red de transporte público (autobuses) en un área urbana. La Estimación de Estado proporcionada por este sistema comprende una estimación automática de la demanda entre estaciones/paradas en el transporte urbano de pasajeros, una estimación de tiempos de viaje y tiempos de operación en las estaciones/paradas, de cargas de pasajeros en los tramos de línea. Como parte integrante del sistema de Estimación de Estado, y como línea futura sería aconsejable el desarrollo de los procedimientos de fusión de estas fuentes de información y su almacenamiento en un sistema de bases de datos como prototipo piloto de un sistema de tratamiento masivo de datos (Big Data) que sirva de soporte a los Operadores del sistema de transporte público.

Esto se ha conseguido mediante el desarrollo de un sistema de detección de dispositivos emisores de radiofrecuencia (wifi o bluetooth) que los pasajeros suelen llevar consigo (teléfono móvil o smartphone). Las aplicaciones desarrolladas han servido para efectuar un seguimiento periódico de forma latente, instaladas en el servidor, o ser invocadas para atender peticiones puntuales las cuales han consistido en:

- Estimación de la matriz dinámica de flujos origen-destino (O-D) de una línea 
transporte, a partir de las muestras generadas por las detecciones de los dispositivos activados.

- Volúmenes de pasajeros, que podían ser 1) puntuales (evolución instantánea a lo largo de un período determinado) 2) volúmenes medios en períodos tipo utilizados en modelos de asignación de pasajeros a líneas de transporte público utilizados para estimaciones de tablas de viajes origen-destino en horizontes temporales amplios, basadas en zonificaciones de transporte propias de estudios de planificación. 3) distribución de las cargas en las líneas.

- Estimación de tiempos de desplazamientos de los pasajeros y de operación de las unidades.

Este sistema ha sido utilizado adicionalmente en entornos docentes con otra finalidad. El sistema proporciona una vista del comportamiento de los usuarios de móvil muy importante, ya que permite inspeccionar los lugares donde se encuentra dentro del complejo que forma la institución docente y el tiempo que se encuentra en ellos en el caso probado de localización en dependencias o aulas de clase.

Permite realizar una estimación muy representativa del tiempo permanecido que dedica cada alumno o empleado de la institución docente. Teniendo siempre en cuenta la pequeña incertidumbre que puede existir en momentos en los que el empleado pueda desprenderse de su terminal móvil de forma puntual o por olvido.

La existencia de un histórico de movimientos, con capacidad de ordenamiento según las distintas variables: empleado, alumno, zona o instante de detección, incluso la representación gráfica donde se distingan las zonas controladas, proporciona una herramienta muy potente para el control, seguimiento y seguridad de alumnos y personal en general de las instituciones docentes de cualquier nivel de enseñanza.

Para validar el procedimiento de detección de dispositivos Bluetooh activos a bordo de un autobús urbano, ha sido necesario calibrar el nivel de ajuste o error y estudiar la precisión en el cálculo de la matriz de flujos (matriz 0-D en su versión dinámica) a partir de la muestra generada por los pasajeros que viajan con su dispositivo wifi o bluetooth activo, y valorar su capacidad de estimación de la carga en tiempo real. Esta validación se llevó a cabo a través de un estudio estadístico y de 
la programación de un software que permitió tener en cuenta las variables y condicionantes que podrían existir para cada línea de autobús, así como los condicionantes externos (meteorológicos, día de la semana, hora, etc.).

Finalmente en la tesis se ha trabajado el diseño y la forma de llevar a la práctica un protocolo cooperativo que permite la extensión de cobertura en redes malladas inalámbricas (WMN). El protocolo incluye mecanismos de ahorro energético para los terminales que actúan de pasarela reenviando mensajes para otros terminales destino a través de nodos cercanos. La ventaja de usar este protocolo es el incremento sustancial de tráfico en la red gracias a las capacidades de extensión de cobertura. Ello es posible gracias a la configuración óptima de los parámetros involucrados en conjunción con el mecanismo descrito de funcionamiento para cada caso posible. El diseño es absolutamente genérico, de tal manera que es posible aplicarlo a cualquier sistema de comunicación clienteservidor-cliente, como es una red WIFI convencional, una red WIMAX o un sistema de telefonía móvil.

Es común que las compañías de telefonía móvil, cuando instalan sus redes alcanzan el $80 \%$ de cobertura de manera relativamente sencilla, pero aumentar esa cobertura hasta el 95\% llega a ser una tarea muy difícil y costosa. El uso de este protocolo además de mejorar los costes generales por transferencia de los datos de ocupación de los autobuses y que ha sido el objeto de parte de este trabajo de tesis, podría conseguir aumentar la cobertura de manera sencilla y rápida. Habitualmente existen localizaciones puntuales en zonas urbanas que carecen de cobertura o conexión con la estación base de la compañía operadora, sin embargo a apenas unos metros de esas localizaciones se posee una cobertura excelente. Para el usuario convencional esto produce una sensación de pérdida de la calidad de servicio, que podría evitarse fácilmente ya que normalmente dentro de un domicilio existen varios terminales que podrían dar una "cobertura virtual" al usuario de manera totalmente transparente, evitando la decepción por parte del usuario al intentar utilizar un servicio que se considera como básico.

Otras aplicaciones del protocolo están relacionadas con la extensión de cobertura o mejora de las comunicaciones en zonas aisladas. Por ejemplo en países emergentes con bajo desarrollo de infraestructuras, o en el despliegue de 
instalaciones de apoyo y colaboración para prestar servicios médicos o de suministros. Otra aplicación interesante sería la prestación de servicios de emergencia en situaciones de catástrofe o bien para el seguimiento de brigadistas en la extinción de incendios forestales [Sanchez'2005]. En este último caso la densidad del bosque imposibilita el seguimiento de la señal GPS por lo que el uso de este protocolo con un software asociado en los móviles de los brigadistas permitiría conocer su situación y movilidad en todo momento desde el camión base, usualmente a unos kilómetros de la acción de los brigadistas. Las aplicaciones son múltiples y de gran interés como el aviso de colisiones múltiples en autopistas del que se ha desarrollado también un prototipo hardware que iría instalado en los vehículos y conectado a los sistemas de aviso del mismo para alertar al conductor en caso de colisión.[ Luque'2011].Finalmente, como otras dos funcionalidades en las que se puede implementar un servicio utilizando el protocolo arriba descrito, se puede pensar en un sistema de asistencia a la conducción por carretera [Milanés'2010] o en un sistema de gestión de servicios de riegos en comunidades de regantes[Amor'2004]. 


\section{CAPÍTULO 6: BIBLIOGRAFÍA Y REFERENCIAS}

[ACM'2015] The Association for Computing Machinery (ACM). The C Library Reference Guide http://www.acm.uiuc.edu/webmonkeys/book/c_guide/index.html.

[Albarracin'2014]Soriano i Albarracin, A. “Estimación de matrices origen-destino en transporte público a partir de la detección de dispositivos Bluetooth.Teoría y simulación". Universitat Politècnica de Catalunya (2014)

[Amor'2004] Amor-Saavedra, Pablo Leon Del. "Sistema De Acceso Movil Para La Gestion De Servicios De Riegos En Comunidades De Regantes". DYNA, junio 2004, vol. 79, no. 5, p.49-52

[Aparicio'2008] S Aparicio, J Pérez, AM Bernardos, JR Casar, “A Fusion Method Based on Bluetooth and WLAN Technologies for Indoor Location," Proceedings of IEEE International Conference on Multisensor Fusion and Integration for Intelligent Systems Seoul, Korea, August 20 - 22, 2008. p. 487-91.

[Bluez'2015] www.bluez.org - Official Linux Bluetooth protocol stack

[Boon'2007] Boon, C.C., Lazarescu, M. and Tan, T. Detection and Monitoring of Passengers on a Bus by Video Surveillance, Proceedings of the International Conference on Image Analysis and Processing (ICIAP), (2007), 143-148

[Bradski'2008] Gary Bradski, Adrian Kaehler, "Learning OpenCV", O'Reilly, 2008.

[Cabrera'2012] Cabrera-Lozoya, A., Cerdan, F., Cano, M. D., Garcia-Sanchez, D., \& Lujan, S. (2012). Unifying heterogeneous e-learning modalities in a single platform: CADI, a case study. Computers \& Education, 58(1), 617-630.

[Caceres'2007] Cáceres, N., Wideberg, J.P.and BenitezF.G. , Deriving origin-destination data from a mobile phone network IET Intell. Transp. Syst., 2007, 1, (1), pp. 15-26

[Cerdan'2014] Cerdan F, Lujan S, et al “Procedimiento De Establecimiento De Conexión Para Redes Inalámbricas". ES Patent. P201030454, ES2391754 B2 (21.05.2014).

[Crispin'2013] John Crispin of the e100boot and boot_linux Linux programs made originally by Axis Communications AB to flash ETRAX 100LX boards under Linux. http://foxlx.acmesystems.it/?id=703\#126.

[Dawei'2008] Dawei Lu, M.S. Route Level Bus Transit Passenger Origin-Destination Flow, Master Thesis. Graduate School of The Ohio State University (2008)

[Ekahau'2015] Ekahau, compañía que comercializa un motor de posicionamiento (Ekahau Positioning Engine 2.1, EPE). http://www.ekahau.com/ 
[Fox'2015] The Fox Borrad, a Linux core engine, http://foxlx.acmesystems.it/foxlx_acmesystems_it/00004/FoxDescripti on_en.pdf.

[Ghazisaidi'2009] N. Ghazisaidi, K. Hossein, and M. S. Bohlooli, "Integration of WiFi and WiMAX-mesh networks," 2nd Int. Conf. on Advances in Mesh Networks, MESH 2009, pp. 1-6, 2009. doi:10.1109/MESH.2009.8

[González'2002] González Castaño F.J., García Reinoso J. (2002). Bluetooth Location Networks. Proc. de IEEE Globecom 2002.

[González'2003] González Castaño F.J., García Reinoso J. (2003). Survivable Bluetooth Location Networks. IEEE International Conference of Communications (ICC 2003). Anchorage, EEUU.

[Guerrero'2007] J.M. Guerrero, J. Matas, Luis Garcia de Vicuna, M. Castilla, J. Miret, "Decentralized Control for Parallel Operation of Distributed Generation Inverters Using Resistive Output Impedance," IEEE Trans. on Industrial Electronics, vol. 54, nº. 2, pp. 994-1004, April 2007.

[Helpy'2015] http://www.helpy.com.ar/Bluetooth/Que-es-Bluetooth.htm

[Hii'2005] P Hii, A Zaslavsky, "Improving Location Accuracy by Combining WLAN Positioning and Sensor Technology," Monash University, Melbourne, 2005.

[Ibeas'2007] Ibeas, Ángel, Manual de encuestas, Ministerio de Fomento de España (2007)

[IEEE'2005] IEEE 802.15.1TM Standard for Information technologyTelecommunications and information exchange between systemsLocal and metropolitan area networks-Specific requirements Part 15.1: Wireless medium access control (MAC) and physical layer (PHY) specifications for wireless personal area networks (WPA N s ) (2005) pp. 559-560

[IEEE802.11] Standard IEEE (Institute of Electrical and Electronics Engineers) 802.11

[IEEE802.15.4] Standard IEEE (Institute of Electrical and Electronics Engineers) 802.15.4

[Khanam'2008] Khanam, Assia, M Z ZShafiq, and M U UAkram, "Fuzzy based facial expression recognition", Proceedings - 1st International Congress on Image and Signal Processing, CISP 2008, Inst. of Elec. and Elec. Eng. Computer Society: (2008). : 598-602, 2008.

[Kim'2011] S.-H. Kim, D.-W. Kim, and Y.-J. Suh, “A cooperative channel assignment protocol for multi-channel multi-rate wireless mesh networks," Ad hoc Networks Journal, vol. 9,no. 5, pp. 893-910, Jul. 2011. doi:10.1016/j.adhoc.2010.10.007. 
[Kostakos'2010] Kostakos, V., Camacho, T., and Mantero, C. Wireless detection of end-toend passenger trips on public transport buses, 13th International IEEE Annual Conference on Intelligent Transportation Systems (2010)

[Kum'2010] D.-W. Kum, J.-S. Park, Y.-Z. Cho, B.-Y. Cheon, and D. Cho,"Mobility-aware hybrid routing approach for wireless meshnetworks," Proceedings, 3rd Int. Conf. on Advances in MeshNetworks, MESH 2010, pp. 59-62, 2010. doi: 10.1109/MESH.2010.18.

[Lee'2009] K.-H. Lee and C. S. Hong, "A PKI based mesh router authentication scheme to protect from malicious node in wireless mesh network," Management Enabling the Future Internet for Changing Business and New Computing Services,pp. 405-413, 2009. doi:10.1007/978-3-642-04492-2_41

[Lobillo'2008] Ramón Lobillo, J. M. Maestre, E. F. Camacho . Sistema de localización mediante tecnología zigbee: aplicaciones a domótica. Actas de las XXIX Jornadas de Automática.

[Lujan'2008] Sergio Luján Fernández, Andrés Cabrera Lozoya, José Fernando Cerdán Cartagena, "Sistema de búsqueda y localización de personas en entornos cerrados basado en dispositivos Bluetooth". VI Telecofórum, Universidad Politécnica de Cartagena. Edición 2008. ISSN 1968-2924.

[Lujan'2011] Lujan, S, et al. "Deeploc: Discreet Indoor People Location Application", New Technologies, Mobility and Security (NTMS), 2011 4th IFIP International Conference on. Piscataway, NJ: Institute of Electrical and Electronic Engineers, p: 1-5.

[Luo'2003] H. Luo, R. Ramjee, P. Sinha, L. E. Li, and S. Lu, "UCAN: a unified cellular and ad-hoc network architecture," in ACMMOBICOM, 2003. doi:10.1145/938985.939021.

[Luque'2011] Luque-Rodríguez, Pablo, Álvarez-Mántaras, Daniel, Wideberg, Johan et al. "Evaluation Of The Road Network: Use Ofa Monitoring Vehicle For The Record Of Potentialy Dangerous Events". DYNA, agosto 2011, vol. 86, no. 4, p.431-437. doi: http://dx.doi.org/10.6036/3990

[Martignon'2009] F. Martignon, S. Paris, and A. Capone, "A framework for detecting selfish misbehavior in wireless mesh community networks," Proceedings, 5th ACM Int. Symp. On QoS and Security for Wireless and Mobile Networks, Q2SWINET'09,pp. 65-72, 2009. doi: 10.1145/1641944.1641958

[Matthieu'2005] Matthieu, JL Crowley, V Devin, G Privat, "Localisation Intra-bâtiment Multi-technologies: RFID, WiFi et Vision," Proceedings of the 2nd Frenchspeaking conference on Mobility and ubiquity computing, pp: 29- 35, 2005.

[Milanés'2010] Milanés-Montero, Vicente, Onieva-Caracuel, Enrique, Vinagre-Jara, Blas et al. "Driving Aid System Based On A Low-Cost Wireless 
Network". DYNA, abril 2010, vol. 85, no. 3, p.245-254. doi: http://dx.doi.org/10.6036/3056

[Montero'2012] Montero, L., Barceló, J., Codina, E. Adapting a Dynamic OD Matrix Estimation Approach for Private Traffic based on Bluetooth data to Passenger OD Matrices, Universitat Politècnica de Catalunya (2012)

[Mood'1974] Mood, A. M., Graybill, F. A., and Boes, D. C. (1974). Introduction to the Theory of Statistics (3rd ed.). McGraw-Hill, New York.

[Murata'1993] Satoshi Murata and Takeshi Hirose, "Onboard Locating System Using Real-Time Image Processing for a Self-Navigating Vehicle", IEEE Trans. on Industrial Electronics. vol. 40,nํ. 1, february 1993145.

[Ollikainen'2002] V. Ville Ollikainen. Bluetooth Applications in New Media Technology. White paper, available [Online] http:// www.cs.hut.fi/Opinnot/Tik86.174/ [October 2002].

[Ortiz'2003] Ortiz G. (2003). El funcionamiento de GPS: un repaso de los principales componentes, tipos de receptores y métodos, [en Internet] $<$ http://www.spain-telecom.net/BoletinInformes2.htm> [27 de octubre de 2003]

[Ortuzar'2011] Ortúzar, J. de D. \& Willumsen, L.G. (2001), Modelling Transport, John Wiley \& Sons (2011)

[Pavon'2005] J. Pavon Mestras, "Programación concurrente con java". Dep. sistemas informáticos y programación, universidad complutense de Madrid. 2005.

[Pediaditaki'2009] S. Pediaditaki, P. Arrieta, and K. M. Mahesh, "A learning based approach for distributed multi-radio channel allocation in wireless mesh networks," Int. Conf. on Network Protocols,ICNP'09, IEEE Computer Society, pp. 31-41, 2009. doi: 10.1109/ICNP.2009.5339701

[Perez'2012] Pérez San-José P. [et al.] Estudio sobre seguridad en dispositivos móviles y smartphones, Instituto Nacional de las Tecnologías de la Comunicación (2012)

[Peterson'2006] Peterson BS, Baldwin RO, Kharoufeh JP, "Bluetooth Inquiry Time Characterization and Selection," IEEE Transactions on Mobile Computing. Vol. 5, no 9. pp:1173 - 1187. Sept. 2006.

[Sanchez'2005] Sánchez Vitores, Raúl. Sistemas de localización en interiores. DYNA, Junio 2005 , vol. 80 , no. 5 , p.30-32

[Spiegel'1982] Spiegel, M. R., Espadas, J. L. G., \& Villasante, A. L. (1982). Teoría y problemas de estadística. Mcgraw-hill.

[Stephen'2008] Stephen B. Miles, Sanjay E. Sarma, John R. Williams, "RFID Technology and Applications". Cambridge University Press. 2008 
[Tesoriero'2008] Tesoriero R, Gallud J, Lozano M, Penichet V, "Using active and passive RFID technology to support indoor location-aware systems," IEEE Transactions on Consumer Electronics. Volume 54, Issue 2.Pp: :578 - 583. May 2008.

[VMware'2015] Referencia de Internet: VMware http://www.vmware.com/es/

[Wang'2011] Wang, Wei, Attanucci, John P. and Wilson, Nigel H.M., Bus Passenger Origin-Destination Estimation and Related Analyses Using Automated Data Collection Systems. Journal of Public Transportation, Vol. 14, No. 4, University of South Florida, 2011

[Wu'2001] H. Wu, C. Qiao, S. De, and O. Tonguz, "Integrated cellular and ad hoc relaying systems: iCAR,” IEEE JSAC, vol. 19, no. 10, 2001. doi:10.1109/49.957326

[Yu'2010] Z. Yu, X. Xu, and X. Wu, "Application of wireless mesh network in campus network," 2nd Int. Conf. on Communication Systems, Networks and Applications, ICCSNA'10, vol. 1, pp. 245-247, 2010. doi:10.1109/ICCSNA.2010.5588704

[Zaruba'2007] Zaruba GV, et al, "Indoor location tracking using RSSI readings from a single Wi-Fi access point," Wireless Networks, vol. 13 (2): 221-35, April 2007.

[Zhao'2007] Zhao, J., Rabbee, A. and Wilson, N.H.M, Estimating a rail passenger trip origindestination matrix using automatic data collection systems. Computer-Aided Civil And Infrastructure Engineering, 22, 5 (2007), 376387

[Zheng'2002] Chunhong Zheng, Licheng Jiao, Zhanting Yuan, Xiping Chen, “A new fast fuzzy processing method for B-Scan image", Proceedings of the World Congress on Intelligent Control and Automation (WCICA) vol 1, issu page 6-9, 2002.

[Zou'2010] F.-M. Zou, T.-S. Wang, X.-H. Jiang, and Z.-X. Lin, “Abanyan-tree topology based railway wireless mesh network architecture," TiedaoXuebao/Journal of the China Railway Society, vol. 32, no. 2, pp. 4754, April 2010. doi: 10.3969/j.issn.1001-8360.2010.02.009 